


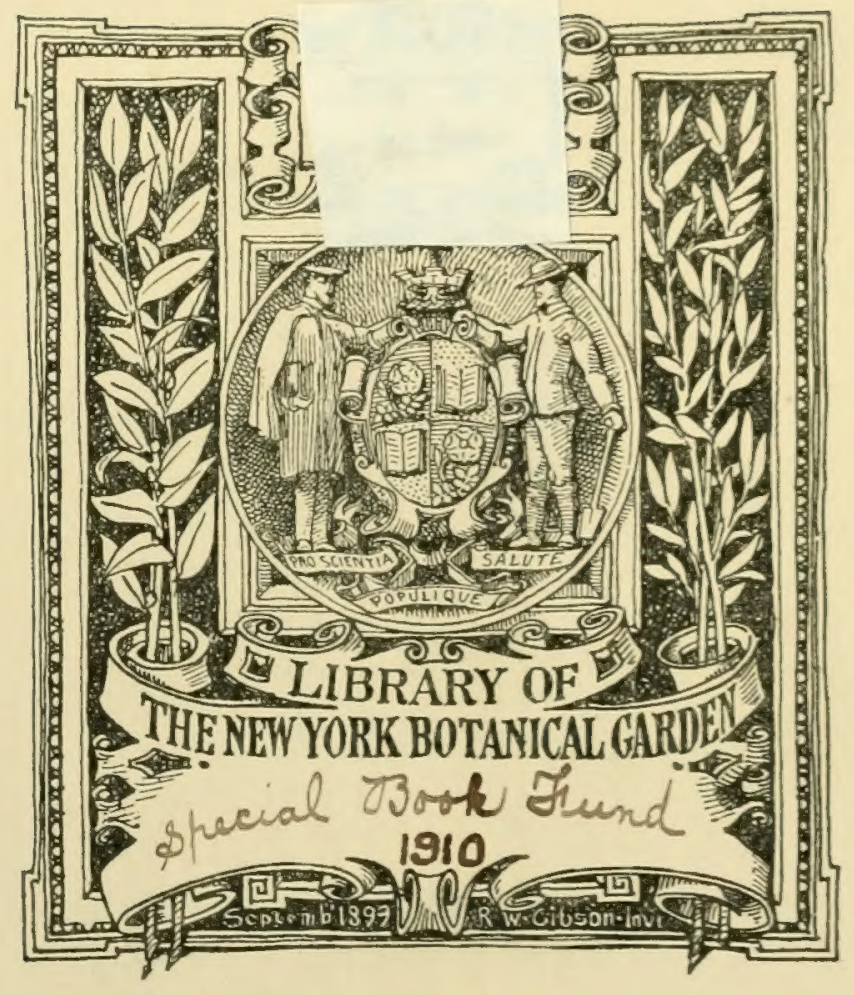






DE I A

\section{nÉTLRHIIITIOY IISTIOLOCGIOLE}

DES

\section{FEUULLES MÉDICINALES}

\section{Par Adrien LEMAIRE,} Docteur en médecine,

Licencié ès-sciences naturelles. - Préparateur de botanique a la Faculté des Seiences de Nancy.

\section{Theodor Oswald Weigel LEIPZIG.}



DE LA

\section{DÉTERMINATION HISTOLOGIQUE}

DES

FEUJLLES MEDICINALES 
(1) 
DE I.A

\section{DÉTDRHIIIITIIOI IISTOLLOGIOLLE}

DES

\section{FEUTLLES NÉUTCTNALES}

\section{Par Adrien LEUAIRE,}

Docteur en médecine,

Licencié ès-siences naturelles. - Préparateur de botanique

à la Faculté des Sciences de Nancy. 

DE LA

\title{
DETERTIINATTON IISTOLOGTQUE
}

DES

\author{
FEUILLES MEDICINALES
}

\section{INTRODUCTION}

L'étude des matières premières végétales employées en thérupentique est entrée depuis plusiour's années déjà dans une voie nouvelle.

La distinction des nombreuses droğues tirées du règne végétal était autrefois uniquement basée sur les caractères de forme, de couleur, de saveur, de dimension. L'examen à l'œil nu n'est point toujours suffisant. Nombreux sont les cas où il est difficile, pour ne pas dire impossible, d'arriver par ces moyens à la détermination exacte des produits purs ou falsifiés. Ainsi sont certaines racines, certaines écorces qui ont entre elles une très-grande ressemblance extérieure. Cette difficulté a suggéré à plusieurs pharmacologistes et naturalistes l'idée de rechercher dans l'observation histologique des organes, des éléments positifs de diagnose. Les résultats qu'a déjar lournis cette méthode ont dépassié ce qu'on avait esperé. 
Les nombreux mémoires et ouvrages qui se rapriortent it cette branche de la pharmacologie inontrent tout le profit que l'on pout tirer' de l'étude des tissus pour distinguer entre eux les nombreux organes végétaux utilisés en médecine.

L'analyse histologique des écorces, des racines, des rhizornes médicinaux est aujourd'lıui très avancée: il n'en est pas de même de l'étude des feuilles qui ont un emploi en thérapeutique. Les descriptions que l'on a données jusqu'ici des tissus de ces organes ne s'appliquent qu'ì un très petit nombre d'espèces, ct sont incomplètes. Les naturalistes qui ont consacré une partie de leur's recherehes a l'examen histologique des feuilles, n'ont tenu compte que d'une portion des tissus de cet organe; ils n'ont fait aucune mention des nervures. Copen-

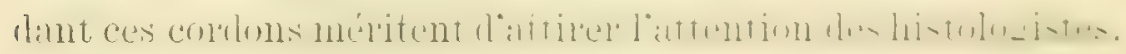
Aucune région de la feuille n'offre en ofret plus de complication dans sa structure intime. Aussi les tissus si différenciés de la nervure peuvent-ils fournir de bons caractères pour la distinction des feuilles.

L'insuffisance des documents, relatifs ì la constitution ana-

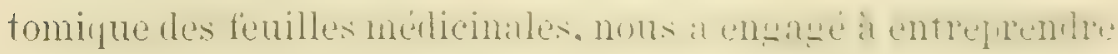
une étude plus approfondie de ces appendices. Nous arons été aussi amené à ce travail par la clifficulté bien connue que l'on éprouve à distinguer les différentes feuilles à l'état où elles se trouvent on droguerie ou en pharmacie.

Si les feuilles assez coriaces et de petite dimension ne subissent point bar la desication de chansement nutable dans leur aspect extérieur, et peuvent par cela même être reconnues par le simple examen du contour, il en est autrement des limbes minces et d'assez grande étendue. Il est rare, en ethet. de renomtrel dans lesollicines, des feuilles qui aient conservé lent interyite parfaite. Elles sont habitullement 
brisées en nombreux fragments ; aussi cet état rend-il impossible une diarnose itablic d'après les caractères tirés de la forme. C'est diuns cette cireonstance yue l'emploi du microscope a une utilité incontestable.

Nos recherches se sont portées sur le limbe foliaire; nous ne nous sommes point occupé du pétiole.

Nous avons divisé notre travail en plusieurs chapitres, de la façon suivante:

I. Histologie générale de Ia feuille.

II. Technique.

III. Description des feuilles.

IV. Clef dichotomique.

V. Conclusions générales

Nous avons fait suivre les conclusions d'une table des matières et d'un index par lettres alphabétiques des genres et espèces que nous avons étudiés.

La fin de notre travail comprend quelques planches qui reproduisent les détails juincipaux d'organisation des feuilles. Ces dessins sont dus au talent de notre excellent ami Abel Thomas, actuellement pharmacien it Saint-Quentin. Qu'il recoive l'assurance de notre profonde gratitude. 



\title{
CHAPITRE PREMIER
}

\author{
HISTOLOGIE GÉNÉRALE.
}

Nous n'avons point l'intention de grouper dans ce premier chapitre les nombreuses connaissances histologiques acquises jusqu'ici par la science sur le limbe foliaire. On trouvera soit dans la plupart des traités de botanique, soit dans des mémoires spéciaux, l'histoire générale des tissus de la feuille ou des autres organes chez lesquels existe une constitution identique, ou à peu de chose près sembla. ble. Notre but est plus restreint; nous nous sommes seulement proposé de classer les divers matériaux que nous a fournis l'observation particulière des feuilles employées en médecine et de leurs falsifications. Nous devons toutefois prévenir le lecteur de ne point chercher dans cette partie de notre sujet, un travail détaillé et complet sur l'histologie si compliquée du limbe des feuilles médicinales. Nous avons donné une assez large extension à l'étude des éléments et des tissus qui, d'une part, sont facilement accessibles à l'examen microscopique, et, d'autre part, fournissent des caractères importants pour distinguer entre elles les différentes espèces: en revanche, nous n'avons décrit que d'une façon très sommaire, ou même avons passé sous silence les détails d'organisation qui n'offrent qu'un faible intérèt pour la diagnose des feuilles.

Ces notions générales pourraient servir en partie de conclusions à notre travail, mais nous avons préféré en faire le sujet de notre premier chapitre. Le lecteur pourra ainsi se rendre mieux compte du sens attribué par nous aux différents termes scientifiques dont il est fait usage dans la description des feuilles de chaque espèce végétale.

Le limbe d'une feuille se présente généralement sous la forme d'une lame verte d'épaisseur et d'étendue variables. On y remarque 
deux faces : l'une supérienre, l'autre inférienre. Le limbe est sillonné de cordons qui le traversent en des sens tries divers et affectent des dispositions variées. C'e sont les nervures.

Tous les cordons qui parcourent une mêrne feuille n'ont pas la même puissance: les uns, assez rolurnineux, font saillie à la face inférieure de l'organe; les autres, très déliés, proéminent à peine. Ils renferment un ou plusieurs faisceaux composés en partie par du tissu rasculaire; tandis que la portion de la feuille située entre cux et à laquelle on peut donner la désignation de lame inleineniale ou extrancurale, en est complètement dépourrue.

Les deux surfaces foliaires sont recourertes chacune d'une pellicule le plus sourent incolore. Ces deux lamelles se réunissent aux deux bords ou maiges de l'organe; elles constituent les deux épidermes. Entre elles se trourent les nerrures et les tissus placés en dehors de ces dernières. La portion de la masse interneurale située entre les delx épidermes, renferme de la matière rerte qui donne à la feuille sa coloration habituelle; on lui donne le nom de mísophylle.

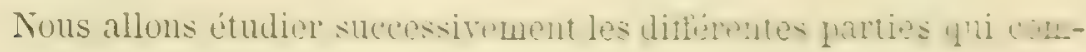
posent la feuille : épiderme, mésophylle, marge et nerrures.

\section{Épiderue.}

L'épiderme, nous tenons de le roir, forme sur les deux faces de l'organe une lamelle incolore. Chaque lamelle est pluricellulaire, et les cellules qui entrent dans sa constitution s'agencent entre elles de façon à ne laisser aucun vide, aucun méat. Plusieurs épidermes, il est vrai, sont percés d'ourertures; mais celles-ci ont une forme particuliope of sont entourdes dielinents dont laspect

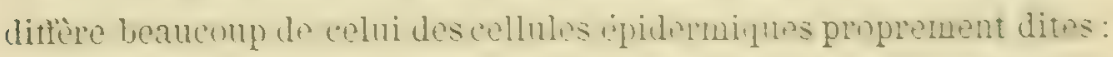
Elles forment avec leur hudure celluhare speciale des appareils dont l'étude nous occupera bientòt; nous roulons parler des stomates.

Le plus grand numbre des esperes posside un ipiderme compusi: d'une assise de cellules. ("est exceptionnellement quil existe denx vu 
plusienrs enuches an moins sur une des faces fuliaires. of enene dans les feuilles qui offrent deux ou plusieurs assises de cellules incolores, il n'est pas toujours facile de saroir par la simple inspection microscopique si les couches profondes appartiennent à l'épiderme ou au tissu intermédiaire. Le déreloppement des tissus peut seul nous fournir des renseignements positifs. On désigne sous le terme de couche de renforcement ou dhypoderme, le tissu incolore sousjacent à l'épiderme, mais ne faisant pas partie de ce dernier.

Les cellules épidermiques sont ordinairement aplaties dans le sens perpendiculaire à la surface de la feuille : elles sont tabulaires. Quand on étale sur un porte-objet un lambeau d'épiderme, la surface des éléments se présente sous des aspects qui varient arec les espèces et dans la même espèce suivant que l'épiderme appartient à la face inférieure ou supérieure de l'organe. Dans les feuilles d'un grand nombre de dicotylédones, les cellules de l'épiderme sont limitées par une membrane qui se plisse de manière à présenter un contour superficiel ondulé. Des portions saillantes alternent avec des parties rentrantes. Les plissements peuvent être peu prononcés, ou, ce qui est le plus commun, atteindre un degré considérable de développement. Les parties saillantes sont tantòt arrondies, comme le montre les figures VII et VIII de la planche II qui représentent les cellules épidermiques des feuilles de Consoude (Symphytum offcinale. L.) et de Belladone (A tropa belladona.L.) ; tantòt aiguës, disposition qui se remarque dans le Datura (Datura stramonizm. L.) (pl. IIl, fig. VII).

Les gymnospermes, la plupart des monocotylédones et quelques dicotylédones sunt pourvus de cetlules épideruiques dont les parois, parallèles à la surface des feuilles, ont la forme de polygones à côtés longitudinaux, droits. Les feuilles de Faham (Angrecum fragrans. Dup. Th.) et de Sabine (Juniperus sabma. L.) présentent des cellules polygonales allongées, suivant la longueur de la feuille, tandis que les diverses cellules de l'épiderme des dicotylédones n'ont point toutes la même direction. L̇xemple: feuille de Ptelée (Plelea trifoliala. L.) (pl. I, fig. V). 
Les collules qui revilent jes plus fortos nerrures sont sujettes id de moindres variations.

Leur contour nest point ondulć. La paroi superficielle forme un rectangle ou polygone dirigé dans le sens de la mervure.

Les cellules épidermiques des deux faces possident généralement la même structure. Tantut, comme cela se remarque dans les feuilles de la plupart des Solanées, des Umlelliferes, les deux Épiderines ont des cellules ì pourtour sinueux; tantòt, au contraire, les éléments sont tous polygonaux. Les feuilles de Sabine (Juniperus sabiua. L.), de Buis (Buxus), de liedoul (Coriarin my,lifoliu. L.) etc., sont des exemples de semblable disposition. Il n'en est pas de même du Tussillage pas-d'àne (Iussilayo farfura. L.) dont les feuilles sont munies d'un épiderme qui dillere sur les deux faces. Les cellules de la face supéricure sont limitées par une paroi nettement ondulée, tandis que celles de la supérieure noffrent aucune sinuosité.

La portion de la membrane cellulaire qui entoure directement la cavité présente habituellement tous les caractères chimiques de la cellulose. L'acide sulfurique et l'iode, employés successirement, ou le chloroiodure de zinc, déterminent une coloration bleue.

Toutes les membranes épidermiques ne manifestent pas toujours les réactions de la cellulose, quelques-unes sont, en totalité ou en partie, transformées en mucilage; c'est ce que font remarquer les feuilles de Buchu (Barosma). Que l'on vienne à placer sous le micros-

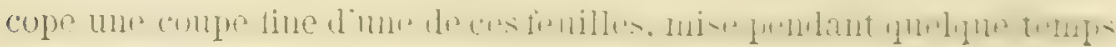

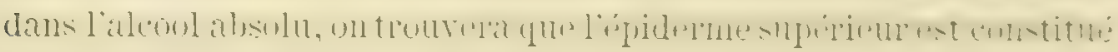
par une couche de cellules. Si l'eramen porte sur des sections placées dans un liquide avide d'eau comme l'alcool absolu ou la glycérine, les cellules de cette assise paraissent très-aplaties et montrent sur leur face profonde, sur celle qui se troure en contact direct arec les cellules du parenchyme vert, une paroi un peu épaissie. Dès que l'on fait arriver de l'eau sur les tranches minces, on roit se gontler

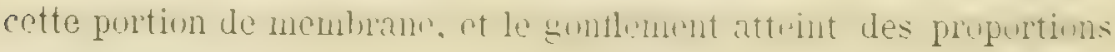
considérables.

La partic superficielle de l'epiderme n est point cellulusique ; elle 
a subi une modifuation que les botanistes ont appelé cuticularisation. Cette portion se montre le plus souvent sous l'aspect d'une pellicule d'épaisseur variable insoluble dans l'acide sulfurique et se colorant en jaune ou en brun sous l'influence d'une dissolution d'iode seul ou de chloroiodure de zine. Dans le Houx (Ilex aquifolium. L.), la culicule ne constitue pas seulement un revêtement superficiel, elle apparait encore sous forme de coin entre les parois latérales des cellules.

La cuticule est ordinairement lisse; mais parfois elle donne naissance à sa surface à des plis ou crêtes peu saillantes qui prennent l'aspect de stries lorsqu'on fait l'examen microscopique d'un lambeau d'épiderme étalé sur le porte-objet. Il suffira de jeter un coup d'œil sur les figures II et V de la planche I, qui représentent, la première l'épiderme supérieur de Tussilage pas-d'àne (Tussilago farfara. L.), la seconde l'épiderme du Ptélée (Ptelea trifotzata. L.), pour se rendre compte de cette production.

La membrane des cellules épidermiques présente une épaisseur très variable. Lorsqu'elle acquiert un certain degré, il n'est pas rare de la trouver parsemée de ponctuations. Ces dernières ne siègent pas indifféremment sur toutes les parois, elles se rencontrent seulement sur les côtés perpendiculaires à la surface de la feuille. On en voit deur exemples frappants dans l'épiderme du Thym commun (Thymus vulgaris. L.) (pl. I, fig. VI) et de la Scabieuse succise (Scabiosa succisa. L.) (pl. III, fig. IV).

Parmi les diverses productions que peut fournir la membrane, nous ne devons pas oublier de signaler les curieux corps appelés cystolilhes f)a. Weddell (1), et que l'on remarque dans l'épiderme d'un grand nombre d'urticées.

L'examen attentif d'une coupe de feuille de Pariétaire (Parietaria officinalis. L.) nous fait reconnaître dans quelques cellules de l'épiderme, plus volumineuses que les autres (pl. IV, fig. VII), des

(1) Weddell. Les cystolithes des urticées, Annales des Sciences naturelles, série IV, tome II. 


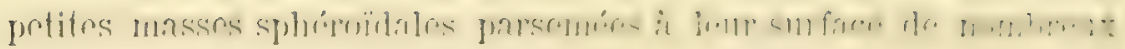
et délicats mamelons. Ces corps ne flottent point libres dans la cavité cellulaire, mais sont ratlachés ì la partie interne de la paroi superficielle par un fin pédicule cylindrique. Vient-on it faire agir sur cux un acide, il se produira une eflervescence et l'apparence tuberculeuse de ces formations disparaitra pour faire place it un petit globe formé de plusieurs conches concentriques.

Traite-t-on ensuite la coupe bien lavée par dn chloroiodure de zinc, le pédicule et le corpuseule des cystolithes se colorent en bleu, ce

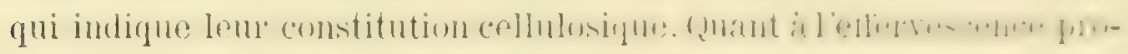
duite par les acides, elle résulte de la présence d'un carbonate (carbonate de chaux) qui incruste la masse de cellulose. Celle-ci

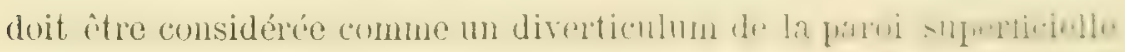
des cellules épidermiques.

Les cellules épidermiques ne contiennent habituellement point de chlorophylle; mais on peut rencontrer dans leur intérieur des substances colorantes diversirs, des matiores huilenses on whenesinenses et de l'inuline.

Les sulustances colorantes autres que la chloruphyllesont it litat de? dissolution. C"est graice à un liquide colnre rentermé dans les cellulur de l'épiderme que certaines fenilles doivent lenr coloration rong.'Il serait, sans contredit, intéressant d'étudier la constitution dess snl colorés et leur répartition dans l'épiderme: mais comme aucun liquidu de cette nature ne se remarque dans les espèces que nous arons étudiées, nous nous sonmes contenté de signaler leur présence pussible dans certaines fenilles.

Les huiles essentielles et les oléorésines peurent se trourer dans l'épiderme de quelques plantes, et dans tous les cléments de cette lamelle. Nous avons constaté la présence de fines gonttelettes cléme sineuses dans les cellules épidermiques de la Pulumenaire ( $I^{\prime}(t) \ldots, \ldots, \ldots$, in officinalis. L.).

Liépiderme diautres fenilles ne posside pas dans tous ses cilenents des subtances oléo résineuses. La production de ces matières est limitée à des cellules spéciales, comme on peut s'en assurer par 


\section{$-11-$}

l'observation microscopique de la fuille d'Asaret d'Europe (Asamm europcum. L.). Entre les cellules épidermiques ordinaires de cette espèce siegent d'autres cellules plus petites dunt la cavité est remplic d'un produit transparent et granulé qui jaunit sous l'action de la potasse et se dissout dans l'éther.

Inuline. - L'inuline, substance voisine de l'amidon par sa composition chimique, est en dissolution dans le suc cellulaire; mais on peut la précipiter à l'aide de réactî́s appropriés. Sous l'inficience de l'alcool ou de la glycérine elle apparaît à l'état de masses douées d'un aspect particulier. L'inuline des feuilles se précipite en effet sous la forme de fines aiguilies, qui se groupent en rayonnant autour d'un point commun pour constituer de petits corps ressemblant à une sphère ou à des fragments sphériques. C'est cette apparence qui a fait donner à ces amas le nom de sphéro-cristaux. Cette matière ne prend aucune coloration sous l'action de l'iode. Elle se précipite fréquemment de sa dissolution contre la paroi cellulaire. Il arrive trèssouvent qu'un puint de la paroi séparant plusieurs cellules contiguös devienne le centre de groupement d'un certain nombre de cristaux renfermés dans la cavité de ces diverses cellules. Il en résulte que l'ensemble prend l'aspect d'une sphère. L'inuline, très-abondante dans certaines racines de composées (Dahlir, Imulr), a été rencontrée par MI. de Lanessan dans les cellules épidermiques de la feuille de Buchu (Barosma.) Nous avons aussi constaté la présence de cette substance dans l'épiderme de la feuille de Ptélée (Plelér trifulinto. L.). que nous avons représenté à la figure $\mathrm{V}$ de la planche I.

De l'épiderme dérivent les formations que l'on a désigruées sous le nom de poils et de stomates.

$$
\text { Poils. }
$$

Les poils sont des appareils filamenteux qui naissent sur l'épiderme et ne sont qu'une dépendance de ce dernier. Les uns portent soit à leur base, soit à leur sommet une cellule ou un groupe de cellules qui ont la propriété de produire des substances oléagineuses : 
ce sont les joils glanduleux; ies autres nont point cotte particnlarité. On appelle ces deruiers joils ondiuraios.

A. Poils ordinnires. - La classification des poils ordinaires adoptée géméralement est bascée sur le nombre, la disprosition et la forme des cellules qui entrent dans leur constitution. Les uns sont unicellulaires, les autres sout composés de plusicurs cellules. Lans ce dernier cas, les cellules sont d'abord placées bout it bout de facon à ne donner qu'une file cellulaire longitudinale, tels sont les joils unisérés; tantòt elles forment plusieurs rangées. Ainsi sont construits les poils nommés pails pluriséviés.

La membrane des cellules peut être lisse ou garnie de tubercules extérieurs plus ou moins saillants. Llle est lisse dans les poils de Belladone (Alropa belladona. L.); tuberenlense dans ceux de Digitale (Digitatis jurpurer. I.) (pl. I, fig. IV, c.), de Séné. La paroi peut aussi être munie de poncluations. Cet état se produit dans la cellule basilaire des poils de Guimauve (Hllhced officinalis. L.). Enfin, comme cela se remarque dans les poils àgés de plusieurs Borraginées, la membrane cellulaire s'épaissit au point de diminuer et même faire disparaitre la carité interne. Quant à la forme des poils ou de leurs éléments, nous en parlerons en étudiant chaque groupe.

I. Poils unicellulaires. - Dans un grand nombre d'espèces les poils sont raides et coniques. Leur base égale ou dépasse en largeur leur portion moyenne. C'est ainsi qu'ils se montrent dans la Mélisse (Melissa ofllcinalis. L.).

Les poils du Baguenaudier (Colutea arborescens. S.). sont au contraire effilés au sommet et à la base (pl. III, fig. VI), ceux de la Pariétaire (Parielarin officinalis. L ) et de la Consoude (Symphylum officinale. L.) (pl. II, fig. VII) se recourbent au sommet; ils ont l'aspect d'hamecon. Enfin, signalons une particularité qui se rencontre

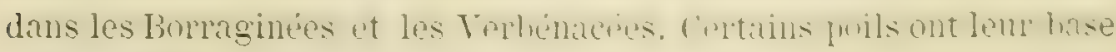
enfoncée daus l'épiderme. Dans les autres plantes le poil est au contraire séparé de la surface épidermique par une cloison transversale. 
II. Poits unisériés. - Les botanistes divisent ces poils en deux groupes, les poils simples et les poils ramifiés.

Poils simples. - Le nombre des cellules qui entrent dans la constitution d'un poil est très variable; mais parfois ce nombre ne dépasse pas une certaine linite. Ainsi les poils de Germandrée petitchêne (Teucrium chanadrys. L.) et d'Hysope (Hyssopus officinalis. L.) sont au plus composés de quatre cellules. D'autres espèces, au contraire, sont recouvertes de poils qui possèdent de quatre à quinze éléments cellulaires superposés.

La cellule terminale de plusieurs poils est conique, tandis que les cellules placées au-dessous du sommet ont en général la forme d'un trone de cône dont la grande base est tournée vers l'épiderme; leur épaisseur diminue de la base au sommet.

Les poils de la Tanaisie (Tanacetum vulgare. L.) ont une structure un peu différente. Leur cellule basilaire cylindrique est surmontéc d'une file de cellules sphériques on ellipsoïdales disposées comme les grains d'un chapelet (pl. III, fig. 1.)

Lorsque la cellule terminale est conique, elle peut être rigide ou molle : elle est raide dans les poils de Digitale (Digitalis purpurea. L.), d'Asaret (Asarum europaum. L.), d'Aunée (Inula helenium. L.), etc.; flexible et très-longue dans les poils laineux ou filamenteux du Tussilage (Tussilago farfara. L.).

Les cellules basilaires d'un poil sont habituellement plus hautes que larges (Digitale (Digilalis purpurea. L.), pl. I, fig. IV c); toutefois certains poils, comme ceux d'Inule (Imele), ont constamment une hauteur moindre que leur diamètre transversal.

Signalons enfin la disposition remarquable des poils de Germandrée petit chêne (Teucrium chamcedrys. L.), de Thym (Thymus vulgaris. L.). Ici les poils, loin d'être droits comme ceux de la plupart des autres plantes, sont fortement coudés au niveau de l'insertion de leur cellule apicale.

Poils divisés ou ramifiés. - Les poils de l'Absinthe (Artemisia (ulsinthium. L.) (pl. I, fig. III, b) constituent le type le plus dégradé de ce gruupe. Ilss sont compusés de deux portions : l'une, fixée à l'épi- 
derme, est perpendiculaire ì celui-ci, cylindrique et formfe d'une rangée de trois ì six cellules; l'autre est horizontale ou un peu inclinée, unicellulaire, fusiforme, et reunic par son foilieu it la partie verticale. Il résulte de cette disposition que les poils de l'Alsinthe Artemisia absinthium. L.) semblent divisés en deux branches égales.

Les poils du Romarin (Rosmavinus officinalis. L.) (pl. II, fig. IV), de l'Origan dictame (Origamm diclammes. L.), ont une tout autre conformation. Ils présentent de distance en distance des branches

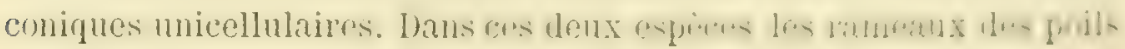
alternent entre eux, tandis que dans la Molène bouillon-blanc ( Ter-

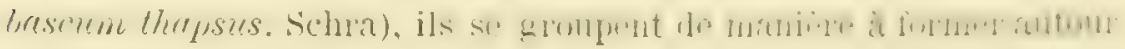
de la partie principale un ou plusieurs verticilles (pl. II, fig. III).

III. Poils plurisériés. - Comme les poils unisériés, les poils plurisériés peuvent être divisés en poils simples et en poils ramifiés.

a. Poils simples. - De nombreuses espèces végétales sont rerêtues de poils simples; mais nous n'arons eu l'occasion de rencontrer de

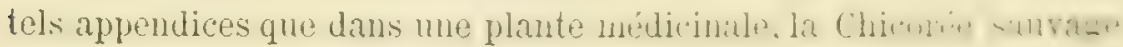
(Cichorium intybus. L.). Les feuilles de ce rẻgétal donnent naissance à de longs poils coniques dont les cellules sont disposées en plusieurs files (pl. II, fig. II).

b. Poits ramifiés. - Les poils ramifiés que nous arons observés dans les feuilles employées en médecine font partie du crulpe lu: poils auquel les botanistes ont domé le nom de puils en rustte. 11: les trouve dans la Guimaure (Allhere ufliciumlis. L.) (pl. IH. fig. T, b, b'), le Boldo (Prenemus boldus. Hol.) (pl. IT, tig. T). La base de ces poils, enchassée dans l'épiderme, n'uffre qu'un petit numbre de cellules, toutes situées sur un seul rang horizontal: du plateau superficicl partent en rayonnant des branches plus on moins nombreuses. Celles-ci sont coniques ot ordinairement unicellulaires. Totons en passant que le nombre de ces rameaux est égal à celui des cellules dont l'ensemble constitue la portion basilaire des poils.

Quand une feuille présente des poils, ceux-ci existent soit sur une. soit sur les deux faces. Dans le premier cas, ils ne siègent qu'ì la face inférieure (Armoise commune Aiteinisiu vulyaris, L.); dans le 
second cas, l'épiderme inférieur produit des poils toujours plus considérables en nombre.

B. Poils glanduleux. - Certains poils portent à leur sommet ou à leur base une cellule ou un groupe de cellules ayant la propriété de sécréter des matères oléagineuses (huiles essentielles, résines, oléorésines). Ces éléments ou ces amas spéciaux sont connus sous la dénomination de glandes, et les poils auxquels elles sont annexées sont des poils glanduleux. Suivant que la glande est supportée ou surmontée par un poil, on dit qu'elle est supère ou basilaire.

Les poils dont la glande est basilaire ne sont point très-communs ; on les rencontre dans l'Ortie (Uitica), la Dictame (Dictammes); mais comme ces espèces n'ont aucun emploi médical, nous laisserons de côté l'examen des poils de cette catégorie pour nous occuper des poils glanduleux à leur sommet.

Ces derniers sont sujets à de nombreuses variations. La glande, les matières sécrétées, le pédicelle accusent de profondes diflérences suivant les espèces végétales.

La glande est tantôt composée d'une cellule unique, tantôt d'un petit amas pluricellulaire. Dans ce dernier cas, les éléments ou bien sont placés sur une rangée horizontale et les cloisons de séparation sont toutes verticales, ou bien ils forment plusieurs rangées cellulaires superposées horizontales ou obliques.

C'est d'après ces caractires que X. Martinet (1) a établi les grandes divisions de sa classification des poils à glande supère.

Quand l'appareil glandulaire est pluricellulaire, quand ses éléments ne sont séparés que par des cloisons verticales, le nombre des cellules quile constituent est toujours un multiple de deux. La glande peut donc avoir $2,4,8,16$, etc., cellules.

Le poil peut être court, moyen ou long; unicellulaire, unisérié ou plurisérié. M. Martinet appelle pédicelle moyen celui qui est formé de deux ou de quelques cellules dont l'inférieure est deux à quatre fois plus longue que large, tandis que les supérieures ont une hauteur au

(1) Martinet. Organes de sécrétion des végétaux. Ann. sc. nat. 5e série, t. 14. 


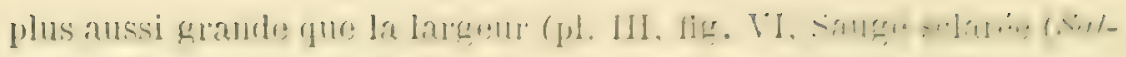
via sclarea. L.).

Quant à la matière sécrétée par les glandes, elle se présente sons l'aspect d'un liquide en forme de globules plus ou moins nombreux; ceux-ci sont tantôt volumineux, tantòt reıarquables par leur peti-

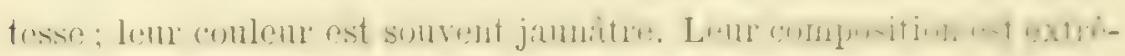

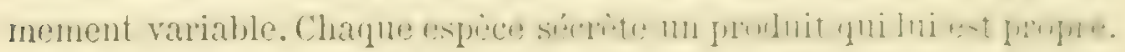

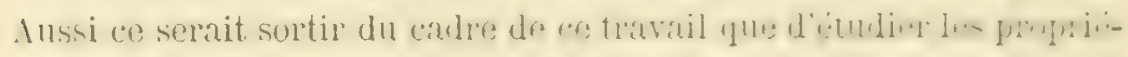
tés chimiques de ces substances si nombreuses.

Nous résumons sous forme de tahleau la classification des poils glanduleux, à glande supère, due à M. Martinet.

Glande unicellulaire.................. Pédicelle court.

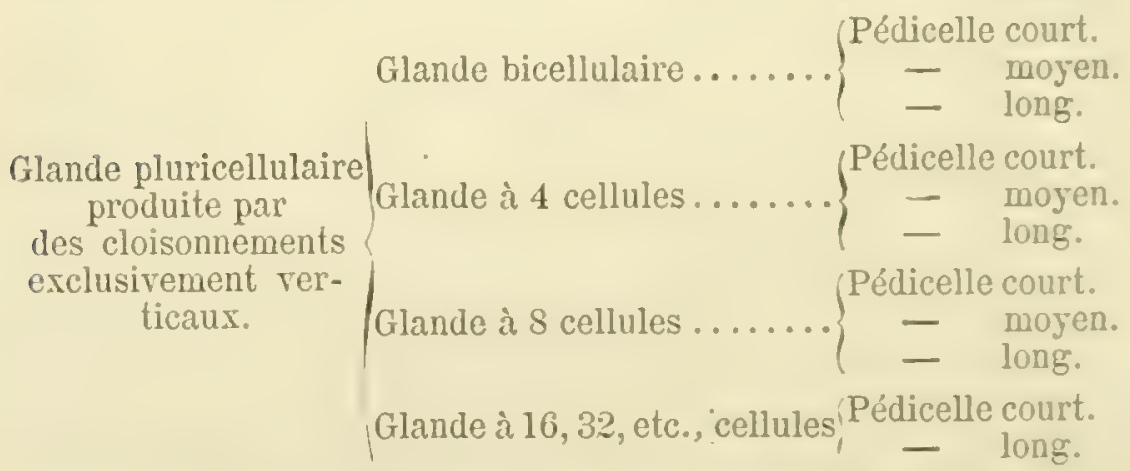

Glande pluricellulaire produite par des cluisome- Pédicelle contrt.

ments non exclusivement verticaux.......... - long.

Gbandes unicellulaires. - Certaines Labiées, Borraginées, Solanées, Scruphularines présentent des glandes de ce genre. Lu mes

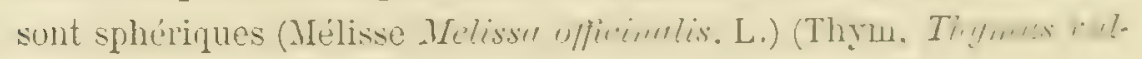
garis), les autres ont la forme d'un œuf (Belladone, Atiopa belladona. L.) (fig. VIII, pl. II).

Le pédicelle est court dans un grand nombre de poils de Labises

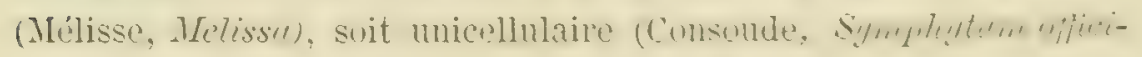
nale. L.) (pl. II, fig. VIl), soit composé de deux ou trois cellules superposées. Il est woyen dans certains sulcie (pl. III, tig. II b, bi) 
Il est long dans la plupart des Solanées (Belladone, Atropa bellapl. II, fig. VIII), dans la Pulmonaire (Pulmonaria officinalis. L.) (pl. II, fig. X).

Glandes pluricellulaires à cellules disposées sur un seul rang horizontal.

a. Bicellulaires. - C'est dans les Labiées, les Verbascées, la Digitale (Digitalis purpurea. L.) que l'on constate la présence de glandes formées de deux éléments séparés par une cloison verticale. Le pédicelle est court, unicellulaire un unisérié dans la Mélisse (Melissa officinalis. L.), le Gléchome (Glcchoma hederacea. L.) (pl. II, fig. I), le Romarin (Rosmarinus officinalis. L.), la Molène (Verbascum), la Digitale (Digitalis purpurea. L.). Il est au contraire allongé dans certains poils que l'on remarque à la face inférieure de la feuille de la Germandrée scordium (Teucrium scordium. L.).

b. Quadricellutaires. - Les poils de ce sous-genre sont ordinairement très courts et unicellulaires. Les cellules glandulaires sont disposées en croix. On en voit des exemples sur les feuilles de Romarin (Rosmarinus officinalis. L.), do Verveine (Verbena officinalis. L.), de Citronelle (Lippia citriodora. Kunth). Nous n'avons point trouvé de glandes à pédicelle moyen ou long.

c. Glandes composées de huit cellules. - Comme les précédentes, les glandes de cet ordre sont le plušs souvent situées au sommet d'un pédicelle cylindrique dont la hauteur est très faible. Leur forme est généralement celle d'un tronc de cône dont la grande base est superficielle; les cloisons qui séparent les cellules rayonnent du centre vers la périphérie.

Les poils glanduleux que nous venons de mentionner s'insèrent le plus souvent au niveau de la surface épidermique; cependant plusieurs glandes appartenant aux deux dernières catégories sont enfoncées dans des dépresions on fussetles plus ou moins profondes que produit l'épirlerme. ('n peut observer de semblables états dans le Thym (Thymus), la Sarriette (Salureia hortensis. L.), l'Hysope (Hyssopus officinatis. L.).

$\mathrm{Si}$ on cxamine ì un fort grossissement, en s'aidant de réactifs 
microchirniques, une glande it 4-8 cellules speciale anx Labiens et anx Verbénacées on est frappé de l'aspectdivers qu'elle présentre pendant le cours de son déveluppernent. Quand elles sont jennes, les glandes sont revêtues d'une couche de cuticule qui s'applique étroitonent sur la nembrane interne de cellulose. L'huile essentielle remplit toutes les cellules. A un àge plus avancé, la sulsstance secrétéc en grande abondance s'extravase entre la partie supéricure de la glande et la cuticule qui la recourre. L'accumulation de liquide qui s'enfectue entre les deux couches de nature chimique différente proroque la distension et le soulèvement de la culicule qui, assez résistante, fait saillie, sous forme de dome au sommet de l'appareil glandulaire; la

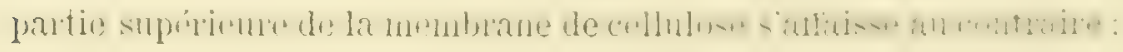
elle s'invagine en quelque sorte dans la portion inférieure de la glande, il en résulte qu'elle prend l'aspect d'une petite coupe dans laquelle repose la masse huileuse enveloppéc supérieurement par la cuticule. La pression exercée par le liquide occasionne finalement la disparition des cloisons et de la cellulose. La glande n'est alors plus représentée que par un sae contenant de l'huile essentielle, et limité par la cuticule.

Glandes à cellules sépariées par des cloisons toules horizontales. - Les exemples de glandes ainsi constituées sont extrèmement rares. M. Martinet en a observé dans la Belladone (Alropa belladona. L.). Nous avors nous-même constaté leur présence dans cette cspèce, mais elles sont peu communes.

Glandes it plusicur's cellules produites par des cloisonnements non exchsivement verlicaux. - Les glandes sont oroïdes (Jusquiame, Hyoscyamus), ( Tabac, Nicotiana tabacum. L.) (pl. III, fig. VIII); clles sont ellipsoïdales (Inule, Inula heleniuin. L.) (pl. II. fig. VI); elles sont coniques (Plantain lancéolé, Planlago lanccolata) (pl. III, fig. III) ou en forme de còne tronqué à grande base supéricure. On en roit un bel exemple dans l'Arnica (Ánicn montana. L.) et la Stramoine (Datura stramonium. L.) (pl.III, fig. VII). Quant au pédicelle, il peut être court et unicellulaire comme dans le Datura (Datuia stranonium. L.) (pl. IlI, fig. VII), la Scabieuse 
succise (Scabiosa succisa. L.) (pl. III, fig. IV), le Plantain lancéolé (Plantago lanceolata) (pl. III, fig. III), ou être composé de plusieurs cellules, tantôt disposées sur un seul rang vertical, tantôt formant plusieurs séries. Certains poils glanduleux de la Jusquiame (Hyoscyamus niger. L.) (pl. III, fig. VIII à ganche), du Tabac (Nicotiana tabacum. L.) sont très longs et unisériés. L'Arnica (Arnica montana. L.) possède des poils plurisériés.

Comme les poils ordinaires, les poils glanduleux peuvent être répartis sur la face inférieure ou sur les deux faces de la feuille. Ils sont toujours plus abondants à la face inférieure.

Les Plantains, les Borraginiess ne présentent jamais qu'une espèce de glandes. Il n'en est pas ainsi des Labiées dont l'épiderme donne naissance non-seulement à de petites glandes unicellulaires ou bicellulaires, mais encore à de volumineux organes glandulaires composés de quatre à huit cellules.

\section{Stomates.}

Ce sont des ouvertures spéciales pratiquées dans l'épiderme. Chaque ouverture est limitée par deux cellules particulières, dites de bordure, et communique avec un espace ou lacune, appelé chambre respiratoire, situé dans le tissu sous-jacent à l'épiderme. Examinons de face un stomate: les cellules de bordure se présenteront sous la forme de deux reins se regardant par leur portion concave et laissant entre eux une fente généralement ovalaire. Une coupe transversale d'une fouille, passant par' un stomate et faite dans une direction perpendiculaire à la longueur de cet appareil, montre un petit canal d'inégal diamètre. Celui-ci, assez large au niveau de la surface de l'organe, se rétrécit insensiblement jusqu'à environ sa mi-hauteur, puis s'élargit de nouveau en infundibulum. Les botanistes ont donné des désignations ì ces direrses parties du canal stomatique: ils ont appelé détroit la partie la plus étroite, chambre antérieure la portion élargie superficielle, et chambre postérieure l'entonnoir profond. 
Les cellules de bordure contiennent habibullement do la chlorophylle; leur cavité, pen considérable, est entourée directenent par une membrane très épaisse. Cette dernière fait défaut sur les parois qui séparent les cellules de bordure des cellules épidermiques ordinaires, mais elle atteint un tris grand développenent aux points qui sont en contact direct arec le wilieu ambiant; aussi subsiste-t-elle it la surface des éléments de bordure et tont antour du canal.

Les Gymnospermes possèdent un appareil plus compliqué: ici, en effet, les cellules de bordure proprement dites sont surplombées par des éléments laissant entre cux un petit espace se continuant arec le canal stomatique. On a réservé le nom d'antichambre à cet espace adventif.

Les rapports des stomates avec les éléments roisins sont très nombreux; nous signalerons seulement la particularité que présentent les stomates de beaucoup de Labiées. Dans le Thym (Thymus vul= garis. L.), par exemple (pl. I, fig. VI), chaque appareil semble être placé vers le milieu de la paroi séparant deux cellules roisines, et de telle sorte que sa fente soit perpendiculaire à cette membrane.

La direction des stomates offre aussi plusieurs variations. Dans la

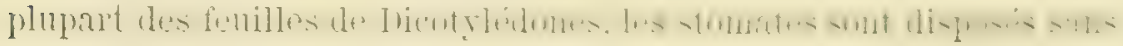
ordre, les uns sout inclinés à droite, les autres à gauche; les uns ont leur ouverture parallèle à la nervure médiane, les autres sont dirigés perpendiculairement à cette nerrure. Dans beaucoup de Monocotylédones et de Gymnospermes les stomates d'un mème épiderme affectent la même direction.

La Sabine (Junipeines salina. L.), comme un grand nombre d'autres Gymnospermes, est munie de stomates qui se groupent pour former ensemble plusieurs bandes longitudinales, séparées les unes

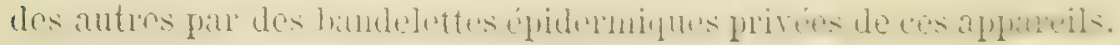

Les stomates ne se rencontrent pas toujours sur les deux faces de la feuille; un grand nombre d'espèces n'en possèdent qu'à la face inférieure de leurs fenilles, tel est le Houx (Ilex aquifolium. L.).

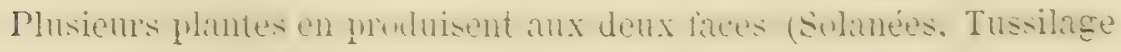

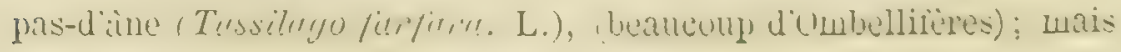


dans ce cas l'épiderme inférieur en présente un plus grand nombre. Enfin la Sabine (Junizems sabina. L.) et d'antres Cupressinées offrent cette particularité remarquable pour des plantes aériennes, qu'clles ne portent de stomates qu'à la face supérieure du limbe foliaire (1).

La forme des éléments épidermiques varie d'une plante à l'autre, clle ne peut servir à caractériser les genres et les familles. Ce caractère ne pent avoir que la valeur de caractère spécifique.

La présence des poils et des glandes n'appartient qu'à un certain nombre de familles. La forme de ces dépendances épidermiques offrant une variété très grande, mais présentant une constance chez les individus de la même espèce, fournit d'excellents caractères pour la distinction des espèces. Parfois l'aspect général de ces productions dans les espèces d'un même genre a la valeur de caractères génériques. Ainsi les différentes Molènes (Verbascum) possèdent des poils ramifiés semblables; les diverses Armoises (Artemisia) sont pourvues de poils qui ont la même apparence extérieure.

\section{Mésophylle.}

Toute la portion de la feuille située en dehors des nervures et recouverte par les deux lamelles épidermiques est en totalité ou partiellement composée d'une réunion de cellules dont la cavité renferme une matière verte, la chlorophylle.

Cette dernière substance, à laquelle la feuille doit sa coloration habituelle, se présente sous la forme de grains assez petits.

Le mésophylle, c'est ainsi que l'on a désigné l'ensemble des éléments cellulaires limités par l'épiderme, est sujet à de nombreuses variations. Pour mieux saisir toutes ses différenciations, examinonsle sous l'aspect qu'il offre le plus ordinairement. Observons, par exemple, au microscope, la coupe transversale d'une feuille de Mélisse (Mclissa offinalis) (pl. V, fig. II). Nous constatons entre les

(1) Nons renvoyons pour de plus complets détuils, aux traités de botanique et it l'important mémoire de Strasburger, in Jahrbücher fur wissen, Botanik $\mathrm{V}, 1866$. 
Épidermes une lamo composcic diun srand nombre de cellules qui contiennent toutes de la chlorophylle. Toutes les collules, il est vai, ne sont point semblables; celles qui touchent l'épidernesupérieur (b) ont à peu près la forme d'un rectangle allongó, perpendiculaire à la surface; clles sont pressées les unes contre les antres. Leur léunion constitue une assise. Les cellules situées au-dessous d'elles sont an contraire arrondies ou ovales et plusieurs sunt parallèles à la unembrane épidermique; elles laissent entre elles de petits espaces ou méats. Les botanistes ont donné au tissu supérieur le now de tissu en palissade, et appelé le tissu placé au-dessous du premier liss? lacuneux, tissu rameux.

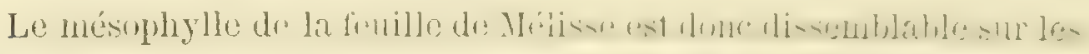
deux faces, il est, suivant l'expression de M. Chatin, hélérogéne et de plus hétérogène asymélrique, parce qu'il n'y a aucune ressernblance entre les tissus qui sont en contact arec les deux épidermes.

La feuille de Sauge sclarée (Salria sclarea. L.) possède un mésophylle un peu différent. 'Tous ses éléments renferment aussi de la chlorophylle, mais ils sont tous perpendiculaires à la surface de la feuille; cependant ils n'ont pas tous la même longueur. A mesure que les éléments se rapprochent de l'épiderme infrienur leur dimension diminue (pl. IV, fig V).

Un troisième type nous est offert par les feuilles de Séné (Cassia) (pl. IV, fig. VI). L'examen microscopique d'une tranche mince montre que le mérophylle est composi. d'une part, de drux assises semblables de parenchyme en palissade dont l'une (c) s'appuie sur l'épiderme

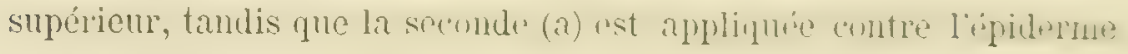
inférieur; d'autre part, d'un tissu intermédiaire (b) aux deux couches ayant tous les caractères du tissu lacuneux. Un tel mésophylle porte le nom de mésophylle hétérogène symélrique.

Le parenchyme vert des feuilles de Faham (Angrocum fiagrans. Iup. Th.) est beancomp plus simple. II ne se littipencie point comme celui des feuilles de Mélisse (Melissa officinatis. L.) en deux sortes de tissus. Les éléments qui entrent dans la constitution de la masse principale ont entre eux une tris grande ressemblance: leurs parois 
sont minces, et ils renferment de la chlorophylle (pl. V, fig. VII). Le mésophylle vert est homogène.

L'épiderme des feuilles d'un grand nombre de plantes recouvre directement la partie colorée par la chlorophylle; mais il peut arriver que les tissus verts soient séparés des cellules épidermiques par une ou plusieurs assises d'éléments qui ont souvent beaucoup d'analogie de structure avec les cellules de l'épiderme. Nous avons déjà eu l'occasion de signaler ces couches spéciales appelées couches de renforcment, couches hypadermiques, hypoderme.

De pareils états se rencontrent dans le Houx commun (Ilex aquifolium. L.) (pl. V, fig. I, b), le Faham (Angracum fragrans. Dup. Thouars).

Nous pouvons résumer toutes les grandes dispositions qu'affecte le mésophylle dans le tableau synoptique suivant :

1. Mésophylle muni d'hypoderme.

2. Mésophylle Parenchyme vert à cellules toutes perpendiculaires.

$\begin{array}{ccc}\text { salls } & - & \text { hétérogène asymétrique } \\ \text { hypoderme! } & - & \text { homogène. }\end{array}$

Etudions maintenant les principales variations de forme, d'arrangement qui s'opèrent dans les divers éléments du mésophylle.

Les cellules hypodermiques des feuilles qui font l'objet de notre travail, ont en général en section transversale la forme d'un rectangle dont les petits côtés sont perpendiculaires à la surface de l'organe. Une coupe longitudinale les montre carrées ou rectangulaires: elles ne contiennent point de chlorophylle et leur membrane, plus ou moins épaissie, a les réactions de la cellulose. Ces éléments s'unissent pour former une, rarement plusieurs assises; il n'existe le plus souvent entre eux aucun méat. Tantôt les deux faces foliaires possèdent un semblable tissu, comme dans le Faham (Angracum fragrans. D. T.) (pl. V, fig. VII), tantòt une des faces en est. dépourvue; la supérieure en présente seule dans le Houx (Ilex aquifolium. L.) (pl. V, fig. I, b), le Boldo (Pnermus boldus) (pl. IV, fig. I, d).

Les cellules du parenchyme en palissade sont, comme nous l'avons déjà vu plus haut, allongées perpendiculairement à l'épiderne; elles 
sont cylindriques, tris rapprochées ies mos des autros. Lemr dimension varie suivant les espèces. Certaines feuilles, comme colles de Mélisse (pl. V, fig. II, b), n'ont jamais qu'une couche de ces cellules. Il n'en est pas de même dans un grand nombre de plantes, telle que le Houx (Ilex aquifolium. L.) (pl. V, fig. I, c), la Cochléaire (Cochlearia officinalis. L.) (pl. V, fig. III, b); ici deux ou plusieurs couches sont constantes.

Quant aux éléments dont l'ensemble constitue le parenchy'ne lacuneux, ils ont un aspect très rariable. Parfois ils sont globuleux ou roisins de la forme sphérique : c'est le cas de la Irélisse (Melissn officinalis. L.) (pl. V, fig. II, c), mais ils sont fréquemment aplatis dans un sens et rameux de l'autre. Les branches de ramification sont courtes ou allongées, affectent une disposition régulière ou irrégulière. Exempl. Cochléaire (Cochlearia officinalis. L.) (pl. V, fig. IlI, c). Les diverses cellules se rénnissent par l'extrémité de leurs rameaux. 11 résulte de cet arrangement de grands espaces intercellulaires. La figure VIII de la planche $T$ donne une juste idée d'un pareil tissu; elle représente une portion de parenchyme foliaire de la Sanicle (Sanicula europce $\ell$. ¿̇.) détachée parallèlement à l'épiderme.

Les éléments du tissu lacuneur sont sourent disposés sans ordre comme dars la Cochléaire (Cochlenirin officinalis. L.) (pl. V, fig. III, c). mais parfois ils se superposent en couches régulières (Mélisse, Melissa officinalis. L.) (pl. V, fig. II, c).

Lorsque le mésophylle est hétérogène, asymétrique, le parenchyme rameux peut être moindre en épaisseur que le tissu en palissade. Il en est ainsi dans la Mélisse (.Molissa officinalis. L.); il peut l'égaler (Belladone, Alropa belladona. L.) (pl. V, fig. VI) ou même le surpasser (Jaborandi, Pilocaipres jennalifolius. Lem.) pl. V, fig. IX, e).

Dans la majorité des cas, le mésophylle est plus puissant que l'en-

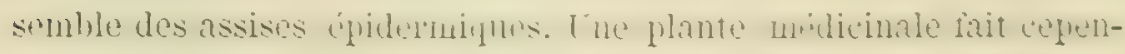
dant exception. L'Oxalide oseille (Oxalis acetosella. L.) possède en efret un épiderme foliaire hauconp phus derehppe que la partie munie de chlorophylle (pl, IV, fig. VIII). 
Les cellules vertes et hypodermiques ne constituent pas toujours à elles seules le mésophylle. Il peut exister, en effet, dans le parenchyme des éléments ou des groupes d'éléments qui se distinguent nettement de la masse environnante, par leur forme, leur épaississement ou leur contenu. Nous voulons parler de ces productions auxquelles les botanistes ont assigné les dénominations de glandes internes, de cellules cristalligènes, d'éléments épaissis.

a. Glandes internes. - Les glandes internes (1) sont des cellules isolées ou des amas limités de cellules qui secrètent des matières oléagineuses (huile essentielle, résines, etc.). On les a divisées en deux groupes d'après le nombre des éléments qui entrent dans leur constitution. Ce sont, $a$, les glandes micellulaires; $b$, les glandes pluricellulaires.

$a$. Les premières, comme l'indique leur nom, ne sont formées que par une cellule; leur contenu est composé d'une ou plusieurs gouttelettes d'huile essentielle généralement colorée en jaune. Lès fenilles de Boldo (Pnenmus boldus) (pl. IV, fig. I, a), de Matico (Artanthe elongata), de Laurier (Laums nobilis. L.), possèdent de semblables organites.

b. Une glande multicellulaire est un petit amas d'éléments cellulaires produisant chacun une certaine quantité de substance oléagineuse. Lorsque la glande est peu avancée en àge, elle présente à son centre, comme à sa périphérie, un plus ou moins grand nombre de cellules, toutes bien distinctes et nettement limitées par une membrane; mais à une période plus parfaite de développement, les collules du centre perdent leur paroi , tandis que les assises périphériques restent intactes et demeurent riches en globules oléagineux; il en résulte un réservir central oi se réunissent les goutelettes provenant des éléments alors résorbés.

(1) Baillon. De la famille des Aurantiacées. Paris, 185.

J. Chatin. Siège des substances actives dans les plantes médicinales. Paris, $18 \% 6$.

Martinet. Loc. cit.

Verne. Etudes sur le Boldo. Paris, 1874. 
Les glandes pluricellulaires sont toujours arrondies: les mnes. comme celles de l'Oranger (Cilmes vulgrias. Riss.) (pl. IV, fig. II, a). sont sphériques; d'autres sont au contraire ellipsoïdales. Celles de Jaborandi (Pilocarnus penualifolius. Lew.) (pl. V, fig. IX, d) sont rangées dans cette catégorie. Plusieurs autres feuilles fournissent des glandes composées. Nous citerons la fenille d'Eucalyptus (Eucr lyplus globulus) (pl. IV, fig. III, d), de Ptéléc (I'lelea lirifoliala. L.). du Buchu (Barosma), de Rue (Rulr).

II. Cellules cristalligènes (1). - Certaines cellules éparses dans el parenchyme foliaire donnent naissance à des substances minérales cristallines. Ces dernières sont toujours formées par de l'oxalate de chaux. L'acide chlorhydrique les dissout sans eflervescence; l'acide acétique n'exerce aucune action sur elles. Ces deux réactions ne se manifestent point avec les dépòts de carbonate de chaux.

L'oxalate de chaux se présente sous des aspects et arec des arran. gements assez divers. Nous n'avons pas l'intention d'entrer dans tous les détails que comporterait une étude approfondie, nous nous

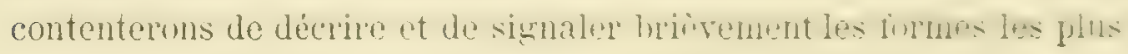
importantes.

a. Cristaux pulvérulents. - Plusieurs plantes de la famille des Solanées offrent dans leurs tissus des cellules oì réside de l'oxalate de chanx sous la forme de grains anguleux très petits chez lesauels

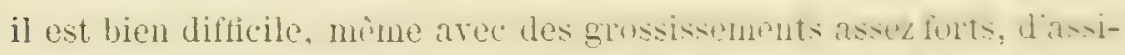

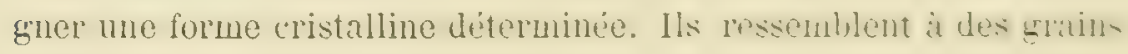
de poussière; aussi leur a-t-on donné le nom de cristaux pulvérulents. On peut en voir un exemple dans la Belladone (Atropa belladona. L.) (pl. V, fig. VI, d).

b. Raphides. - On comprend sous ce terme des cristaux en forme d’aiguilles déliées, groupés cute à coite en faisceanx dans tonte la cavité de cellules spéciales. Le paquet cristallin est griniralement enreloppé par une masse mucilaginense qui se gonfle lursque leilement

(1) Vesque. Des cristaux d'oxalate de chaux dans les plantes. Ann. st. nat. Botanique, série VI, t. II, $18 \%$. 
cristalligène est mis au contart un l'ara. Ce gonflement exerce sur la membrane cellulaire une pression qui aboutit à la rupture de la paroi et finalement à l'évacuation des cristaux. Les éléments à raphides se rencontrent fréquemment chez les Monocotylédones.

c. Ciristaux isolés. - Ils se montrent sous l'aspect de cristaux très-visibles : tantôt prismatiques, tantòt en forme d'octaèdre. La cavité cellulaire peut ne contenir qu'un cristal ou plusieurs cristaux. mais dans ce cas ils sont toujours isolés les uns des autres. Les feuilles d'Oranger (Citrus vulgaris. Kiss.) produisent dans leur mésophylle une grande quantité de cellules à cristaux isolés (pl. IV. fig. II, b).

d. Crislauxagglomérés. - On appelle ainsi des groupes ou macles de cristaux d'oxalate de chaux. On en rencontre dans le parenchyme vert des feuilles de Groseillier (Ribes nigrm. L.) et d'Eucalyptus (Eucalyptus globulus) (pl. IV, fig. III, c).

Les cellules qui renferment des cristaux sont généralement dépourvues de chloruphylle; toutefois certains végitaux peu nombreux. parmi lesquels se range la Jusquiame, possèdent de la matière verte dans leurs organites cristalligènes.

Il s'en faut de beaucoup que toutes les plantes produisent de l'oxalate de chaux dans l'intérieur de leurs cellules. Les végétaux appartenant à quelques familles ont leurs feuilles privées de cette substance: ainsi les feuilles médicinales de Labiées, Scrophularinées, Synanthérées se font remarquer par l'absence de cristaux.

Quand ceux-ci existent, leur forme, leur disposition sont assez constantes chez les individus de la même espèce pour qu'on leur accorle une grande valeur dans la détermination des espèces.

III. Cellules isolées à membrane épaissie. - Le mésophylle de quelques feuilles contient dà et là des éléments isolés qui tranchent sur le tissu général par leur aspect extérieur et par l'épaississement de lenrenveloppe. Le parenchyme de la fenille de Faham (Angreerem rragrans. Dup. Th.) est parsemé d'éléments $a, a^{\prime}, a^{\prime \prime}$ (pl. V, fig. VII et VII, b), qu'une section transversale montre circulaires, tandis qu'une coupe longitudinale les fait voir sous la forme de fuseaux assez longs 
Le The (Thea chinensis. I..) (pl. IV, fig. IV) presente des cellules". qui s'étendent dans le massif sous-épidermique. Ils produisent do: courtes ramifications qui n'affectent aucune régulariti dans lour disposition. Leur paroi collulaire très épaissie se colore en brun sons l'action de l'eau iodée; clle est lignifice.

Si l'on compare la masse principale du inésophylle dans les plantes de la même famille, on est frappé de la grande diversité de structure que présente cet ensemble de tissus et l'on se pénètre de l'idée que ce dernier ne peut servir à caractériser les familles.

Mais si le nombre des assises cellulaires et l'agencement des élements verts varient d'une plante à l'autre, ils offrent cependant une constance remarquable dans les feuilles appartenant aux dirers individus d'une même espèce. Quelquefois la constitution du mésophylle ne subit aucune modification notable chez les espèces d'un mème genre. Le genre Cassix, représenté par trois espèces médicinales, en est un bel exemple. Les feuilles de ces trois espèces possèdent un parenchyme vert hétérogène symétrique analogue.

Les éléments ou les groupescellulaires, dits aceidentels, fummi-sint au contraire des caractères d'ordre élevé. Leur présence est habi-

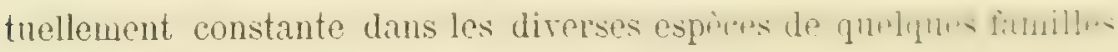
végétales; aussi les organites peuvent-ils être mis au rang de caractères de famille. Toutes les Myrtacées. tontes les Rutacrips qui mut lait lobjet de notre étude sont pourvues de glandes internes. Lims arums eu déjà l'occasion de signaler la valeur des cellules cristalligènes dont l'absence dans un certain nombre de familles sépare ces dernières d'autres assez voisines. Les fenilles des Labiées, Borraginées, Synanthérées, etc., manquent constamment d'éléments à cristaux.

\section{Marge.}

Dans la plupart des plantes les bords latéraux de la feuille sont constitués par du tissu épidermique contre lequel vient sippryer directement la portion terminale, les tissus verts. Li figure II de la planche V, tirée de la Jusquiame (Hyoscyamus niger. L.), peut donner une idée des tissus marginaux. 
- Quelques espices offrent cependint contre l'épiderme des bords un massif d'éléments qui diffèrent de ceux du mésophylle par leur aspect particulier. Que le lecteur veuille bien jeter un coup d'œil sur la figure $\mathrm{V}$ de la planche $\mathrm{V}$ représentant une section transversale de la région latérale de la feuille d'Airelle rouge (Vaccinium vitis idce. L.), il verra en $a$ un petit groupe d'éléments polygonaux dont la membrane est très épaisse.

Ces caractères, dont, je crois, on n'a pas tenu compte jusqu’alors, peuvent être très-ntiles pour différencier les unes des autres quelques espèces roisines.

\section{Nervures.}

On sait que le limbe foliaire est parcouru par des cordons qui proéminent d'ordinaire à la face inférieure de l'organe. Ces cordons offrent des dispositions très variées.

La nerration assez simple dans certaines espèces, peut chez d'autre atteindre un très haut degré de complication.

Ce dernier état nous est offert, par exemple, par la Digitale. Une nervure très saillante, partant de la base de la feuille et se dirigeant vers le sommet, partage l'organe en deux parties latérales, souvent symétriques.

Tout le long de cette nervure, appelée nervure principale, s'échappent à droite et à gauche des nervures plus petites, secondaires, qui, à leur tour, se ramifient un très grand nombre de fois, de facon à constituer finalement un réseau complexe. Toutes ces nervures n'ont point la même puissance.

Le cordon médian diminue d'épaisseur au fur et à mesure qu'il se rapproche de l'extrẻmité de la feuille.

Les autres nervures, moins développées que la principale, sont d'autant plus faibles qu'elles sont les branches de ramification plus élevée.

Si l'examen superficiel fait comnaitre les changements de volume qui se manifestent dans les différentes nervures, l'inspection microscopique nous apprend aussi que les tissus subissent des variations. 
Non-seulement les assises cellulaires deviennent moins nombrenses. mais encore certains élements anatomiques disparaissent dans les nervires délicates.

On serait tenté, en raison de cette variabilité de structure de n'accorder aucune valeur à l'étude histologique des nervures. Sans doute, si on dirige au hasard ses observations, si on examine an microscope des sections pratiquées sur les nerrures d'une inếne plante en des points très éloignés les uns des autres, on trouvera de très grandes différences d'organisation; rnais, que l'on fasse des coupes minces dans plusieurs feuilles de la même espèce, sur la même saillie neurale, et, ¿̀ peu près au wème niveau, on sera alors frappé de la grande ressemblance qui existe au point de rue miscroscopique entre toutes les tranches.

Nous nous sommes toujours servi de la nervure médiane, soit que la feuille ait une nervation pennée ou palmée, soit qu'elle possède des nervures paralleles, cumne cela se remarque dans lwanculp le monocotylédones.

Les feuilles sont-elles longues? nous arons produit nos sections transversales à environ un centimètre de la base de l'organe. Dans les feuilles petites, nous les arons faites à un ou deux millimétres.

Nous arons choisi de préférence ces points parce qu'il est toujours facile de reconnaitre, même au milieu de nombreux fragments de

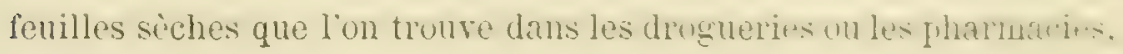
des morceaux qui appartiennent à la base des feuilles.

La nelrure présente une structure beancoup julus compliphra yne: les autres parties de la feuille.

Outre l'épiderme qui la revêt aux deux faces, outre une masse parenchymateuse, principale, tris complexe. on y trume nu ensemble

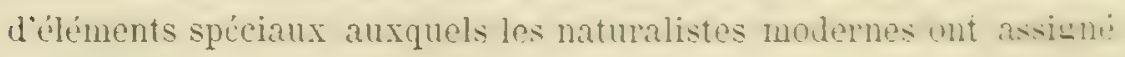
la dénomination de systène fibro-vasculaire, système libéro-ligneux.

L'épideme neural offre la mème structure générale que celui qui recourre la mésophylle. Toutefois les cellules, au lieu d'ètre sinucuses affectent la forme de rectangle on de polygone allongé dans la direction de la nervure. Les stomates sont moins numbreux et 
même n'existent pas. A part ces quelques variations, l'épiderme pent derenir le siege de productions comme les poils, les poils glanduleux, productions qui sont généralement plus abondantes sur cet organe.

Système libéro-ligneux. - Le système auquel les botanistes ont réservé le nom de système libéro-ligneux se rencontre sous l'aspect de cordons plongeant dans une masse cellulaire située entre les deux lames épidermiques. Ces cordons ou faisceaux, en raison de la structure et de la largeur des éléments beaucoup plus petite que celle des cellules environnantes peuvent se séparer très facilement de ces dernières. Leur nombre est assez constant au même niveau de la nervure. Tantốt ils sont très écartés les uns des autres, de façon a être reliés par un massif celluleux assez considérable. Tantòt la distance qui les sépare est très faible au point qu'il ne sont éloignés les uns des autres que par quelques rangées cellulaires. Quoi qu'il en soit, ces faisceaux se disposent habituellement de manière à constituer ensemble une bande courte dont la concavité regarde la face supérieure de la feuille tandis que la convexité est inférieure. Exemple: Epine vinette (Berberis vulgaris. L.) (pl. VII, fig. IV, A), Mauve (Malva), Labices. Daus la plupart des feuilles les extrénites latérales de l’are sont très écirtées les unes des antres comme dans la Belladone (1tioyn belladona. L.) (pI. VI, fig. V); mais, dans d'autres, cet are est tellement courbé en fer à cheral que les extrémités sont très voisines et finissent ruime par se tuncher. Ln exemple d'une semblable disposition se rencontre dans l'Alchemille (Alchemilla vulgaris. L.)

Certaines plantes, peu nombreuses, présentent outre une bande à concavité supérieure un arc dirigé en sens inverse.

Il en est ainsi dans le Sumac vénéneux (Rhus toxicodendron. L.) (jl. VII, fig. III).

Enfin les cordons libéro-ligneux peuvent se disposer suivant plusienrs lignes courbes naffectant ancun ordre. Celte particularité est extrèmement rare; nous la signalerons dans le Tussilage pétasite (Tussilago petasiles. L.).

Il ressurt de ces differents arrangements que les faisceanx foliaires ne sont pas placés comme ceux de la plupart des tiges et des racines 
suivant une ligne circulaire. Il ne sont pas silues syondriqueurent par rapport à un axe passant par le centre de l'organe. Ils ont au contraire une symétrie bilatérale.

Etudions maintenant la constitution de ces faisceaux. Charne faisceau foliaire est composé de deux groupes d'úléments particnliers auxquels on a donne les noms: 10 de liber: 20 de bois ou de ligneux. Ces deux groupes se trourent superposés de la facon sulvante. Le liber est placé sur le côté externe du bois; anssi quand les cordons forment ensemble un seul are ì convexité inférieure, le liber regarde la face inférieure, tandis que le bois est tourné vers la portion superieure de l'organe. Lorsqu'à l'arc nornal vient s'ajouter une bande à convexité supérieure, cette dernière a évidemment son liber tourné vers l'épiderme supérieur.

a. Liber. - Quand le liber atteint son plus laut degré de perfection, il est formé de:

$1{ }^{\circ}$ Cellules libériennes ou séreuses;

$2^{\circ}$ Cellules et tubes cribreux ou grillagés;

$3^{*}$ Cellules cristalligènes;

4. Fibres libériennes.

Il nous faudrait ajouter à ces éléments la liste des organites assez nombreux, comme les glandes, que l'un peut aussi remuntre ulau- l." liber, mais leur étude fera l'objet d'un paragraphe spécial. La réunion des trois premiers éléments que nous renons d'énumérer constitue ce que l'on est conrenu d'appeler le liber mou, ou celluleux pour le distinguer de la portion composée de fibres nommés liber fibreux, liber prosenchymateux ou parfois liber dui".

Les cellules séveuses sont des éléments assez petits. Leur base droite ou un peu oblique a l'aspect d'un carré ou d'un polygone à quelyues cotés: leurs membranes. plus un muins equi-sies, wht tonjours la réaction de la cellulose.

Les parois tangentielles antérieures et postérieures sont lisses, andis que les parois transrorsales et latemales sunt munies de punctuations. Ces éléments ne laissent entre eux aucun weiat. ils se pla- 
cent bout ì bout pour former des files longitudinales plus ou moins étendues. Ces cellules ne manquent jamais.

Les éléments grillageux ou cribreux sont ainsi nommés parce que leurs parois transversales supéricures et inférieures sont percées d'un grand nombre de petites ouvertures, qui donnent à ces parois l'apparence de cribles ou grillages. Les botanistes distinguent deux sortes d'éléments cribreux, ceux dont les parois transversales sont sensiblement horizontales ou peu obliques, ce sont les tubes cribreux, et ceux dont les mêmes parois sont plus ou moins obliques, ce sont les cellules grillagées proprement dites.

Les éléments cribreux et séveux ne contiennent point de chlorophylle mais sont remplis d'une substance de nature albuminoïde.

La structure délicate de toutes ces cellules n'est puint très facile ì distinguer sur les feuilles sèches. Les préparations que l'on est obligé de faire subir à ces organes pour en rendre l'étude microscopique plus commode, altèrent profondément, non point la forme des cellules libérienues, il est rrai, mais la constitution de leurs membranes. Aussi avons-nous omis, dans notre étude particulière des fenilles médicinales, la description détaillée de ces éléments libériens. Nous nous sommes souvent contenté de signaler la présence du liber mou sans indiquer les diverses sortes de cellules qui entrent dans sa constitution.

Quant aux cellules cristalligènes, ce sont de petites cellules cubiques on prismatiques, à base rectangulaire ou carrée; clles renferment des cristaux le plus souvent agglomérés d'oxalate de chaux. Elles jouissent de la propriété remarquable de se disposer en files verticales.

Les fibres libériennes, éléments allongés terminés en pointe à leurs extrémités, sont pourrues d'une membrane dont l'épaisseur et la constitution chimique présentent des variations très importantes. La paroi, mince dans certaines Solanées, est au contraire épaissie dans les fibres du Houx, (Ilex aruifolium. L.) du Laurier cerise (Cerasus lauro-cerasus. L.). Tantôt, le chloroiodure de zinc la colore en bleu, tantôt au contraire ce dernier réactif produit une coloration brune 
earacteristique du lignoux. Les fibres ì parrois lignifiees ne sont jorint rares. On les rencontre dans lo Holix (Iles: armifiliume. L. ), Lanrier cerise (Corasus laumo-cerresus. I..), etc.

Les divers éléments qui composent le liber penvent se ranger de

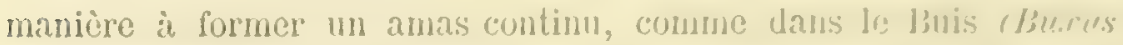
sempervirens. L.) (pl. VI, fig. I (c, fibres libériennes - d, liber crellulenx); mais dans certains cas fournis, par exewple, jar les solanées, le liber est divisé en petits groupes on ilots, séparés les uns des autres par une ou par quelques rangées do cellules assez volumineuses. La figure $V$ de la planche VI représente en $b$ un de ces ilots et en $a$ une cellule placée entre les doux massifs.

Le liber peut être purement celluleux (Redoul (Corirnir mu, rlifolia. L.) (pl. VI, fig. II, a) ou cellulo-fibrcux. I)a.as ce dernier cas los fibres peuvent former une lame continue située à la périphérie du liber celluleux (Buis, Buxus sempivinens. L.), ou se disposer en petits groupes, comme dans le Laurier cerise (Ceiasus lauio-cerasus. L.).

La forme du liber est aussi très variable. Le julus somrent, il se montre sur une coupe transversale de la nervure, sous la forme d'un arc concave vers la face supérieure de la feuille de Buis (Buxus seingrevirens. L.), (pl. VI, fig. I, c et d, etc.); mais parfois, comme on peut s'en rendre compte sur une section pratiquée à travers la base de la nervure d'Aconit (Aconilum napellus. L.), le liber constitue un amas biconvexe (pl. VIII, fig. II, d).

Nous avons vu que le liber était toujours adossé à la portion du bois la plus voisine de la périphérie; toutefois la partie ligneuse des laisceaux peut être tlanquée sur ses deux faces opposées d'une partic libérienne. Il y a non-seulement liber externe, mais encore liber interne. Cette disposition, signalce pour la première fois par Sanio en 1864 sur les rameaux de Tecoma radicans, a été étudiée depuis, surtout par M. Vesque (1), sur un grand nombre de tiges.

(1) J. Vesque. Anatomie comparéc de l'écore. Ann. sc. nat., fe serie, t. II, $18 \%$ 
Le tableau suivant en énumère les principales variations.

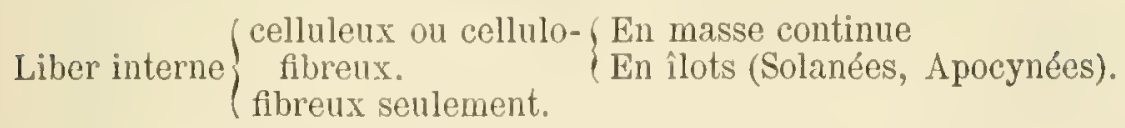

Bois. - La partie ligneuse des faisceaux est caractérisée par la présence de vaisseaux auxquels peuvent s'ajouter des fibres et des cellules ligneuses.

Les vaisseaux offrent des aspects très divers. Ceux qui ne manquent jamais sont les trachées. Quand le bois est formé d'un mélange de différents vaisseaux, les trachées se trouvent toujours situées à la portion interne du lignenx. Uutre les trachées, le bois peut renfermer des vaisseaux spiraux, annelés, réticulés, ponctués, etc., dont on trouve la description détaillée dans les ouvrages de botanique.

Les fibres ligneuses se rencontrent rarement dans les faisceaux foliaires. Ce sont des fibres à paroi épaissie, ponetuée et lignifiée. Nous en avons vu dans la Pervenche (Vinca minor. L.). Chez les Gymnospermes qui sont privées de vaisseaux à l'exception des trachées, les fibres forment la partie la plus importante du bois : celles-ci ont une structure particulière aux plantes de ce grand groupe des Phanérogames; elles sont munies de ponctuations aréolées circulaires. La figure IV de la planche VIII montre de semblables ponctuations (Sabine, Juniperus sabina. L.).

Quant aux cellules ligneıses, elles ont généralement une enveloppe mince cellulosique.

Les éléments ligneux affectent très souvent une disposition régulière; ils se groupent en rangées rayonnantes. On en voit un bel exemple dans le Buis (Buxus sempervirens. L.), pl. VI, fig. I, e; la Mauve (Malva rolundifolia. L.), pl. VII, fig. I, f. Tantôt au contraire aucun ordre ne préside à leur arrangement. Les vaisseaux de la feuille de Tussilage (Thssilago farfara. L.) rentrent dans cette catégorie (pl. VII, fig. V, b).

Système fondamental. - Les botanistes allemands ont les premiers appliqué la dénomination de systeme fondamental au tissu on ¿ l'ensemble des tissus qui se trouvent enveloppés par l'épiderme et 
qui nappartionnent point an systirne libern-lignenx dont nons venons d'esquisser l'histoin'e.

Un grand nombre de naturalistes s'accordent it ctablir dans ce système les trois grandes divisions suivantes :

$1 \circ$ Hypoderme;

$2^{\circ}$ Couche protectrice des faisccaux libéro-ligneux;

$3^{\circ}$ Tissus de remplissage.

L'Hypoderme est formé d'un ensemlile déléments dont la disposition, la forme et la structure sont assez tranchées; il cst généralement sous-jacent à l'épiderme, d'où son nom. Jous arons déjà eu l'occasion de trouver le tissu hypodernique adossé à l'épiderme de la portion foliaire interneurale; nous arons ru qu’il était constitué par une ou plusieurs assises de cellules incolores dont les parois sont d'ordinaires assez minces.

L'hypoderme de la nervure ne présente pas toujours cette simplicité d'organisation. Les éléments qui entrent dans sa composition ont un aspect très virié. Tantót ce sont des cellules dépourvies de chlorophylle, allongées en forme de prismes droits à base carréc. ou rectangulaire et dont la membrane d'enreloppe est épaissic également sur toute sa surface. Cette membrane, lisse ou ponctuée, a les réactions de la cellulose. La feuille du Laurier cerise rejosus la?!o cerams. L.) en est un exemple. Tantòt les cellules, tout en étant longuement prismatiques comme les précédentes, oftrent cette particularité remarquable de n'épaissir leur paroi qu'aux angles. Un a rúservé le terme de Collenchyne au groupement de cellules scaublables. Il y en a deux types: Le Collenchyme contiac que lon rencontre chez les IIalvacées, ou l'épaississement forme vers l'intérienr de la cellule une forte saillie arrondie pareille à une colome cngagée; et le Collenchync concare ou l"épaississement faible n'empiète point sur la cavité cellulaire. Plusieurs Borraginées sout munies de ce tissu.

Tantòt enfin l'hypoderme est représenté par des éléments très longs amincis en pointe aux denx extrémités, et dont la paroi 
épaissie est lignifiée. Les Gymnospermes possèdent en général cet hypoderme prosenchymatenx.

L'hypoderme est le plus souvent continu, mais parfois il constitue de petits groupes placés ì une certaine distance les uns des antres. L'hypoderme du Matico (Artanthe elongaln) (pl. VI, fig., II b) rentre dans cette catégorie.

Ce tissu peut exister aux deux faces de la nervure, alors l'épaisseur de l'hypoderme est habituellement plus considérable à la face supérieure (Guimauve, Althaca officinalis. L.) (pl.VII, fig. I, d, II). Il peut manquer à l'une des faces, tantòt à l'inférieure, tantòt à la supérieure. Enfin, il peut faire totalement défaut comme dans le Buis.

E Couche protectrice des faisceaux. - On désigne ainsi une assise particulière de cellules enveloppant chaque cordon libéro-ligncux ou tout le système fasciculaire d'un organe. Cette conche, dont le type nous est oflert surtout par toutes les racines peu àgées, ne se montre qu'exceptionnellement dans les feuilles, et cncore est-elle loin de présenter les caractères aussi nets que ceux que l'on remarque dans les racines.

Les éléments protecteurs ne présentent plus sur leur paroi latérale ces plissements longitudinaux caractéristiques que l'on prendrait volontiers, si on observe une section transversale de ces cellules, pour des épaississements. Ils sera pprochent généralement de la forme rectangulaire ; leur membrane est également mince, leur contenu est incolore; enfin, adossés par leur paroi latẻrale, ils constituent une assise cellulaire entourant le faisceau. Grâce à la position, la forme et l'agencement de ses cellules, toujours différant des éléments environnants, cette couche doit être regardée à juste titre comme l'homologue de l'assise protectrice que l'on observe autour du système libero-ligneux des racines. Il suffira de jeter un coup docil sur la fig. II, pl. VI, représentant la coupe transversale de la nervure du Rédoul (Coriaria myylifolia. L.' poul' se faire une idée de cette zòne. Celle-ci est figurée en $c$.

Tissus de remplissarje. - Les naturalistes donnent le nom de tissus de remplissage à toute la portion du système fondamental qui 
n'est ni hypoderme, ni conche protectrice. Qmand des cordons libéroligneux forment deux bandes courbes, l'une supérieure, l'autre infirieure, se regardant par leur concavité, le tissu de remplissage est nécessairement séparé en plusicurs portions:

1. Une partie qui se trouve ì la périphérie du systime fasciculaire. Nous pouvons l'appeler massif jéniphérique; elle représente le tissu cortical des tiges et des racines;

$2^{\circ}$ Une portion occupant l'espace produit vers le centre de la nervure par les deux ares libéro-ligneux; c'est la molle;

$3^{\circ}$ Une partie située dans l'intervalle que laissent entre eux les faisceaux, partie reliant les tissus périphériques à la moelle, ce sont les grands rayons médullaires.

Lorsque le système fasciculaire n'est qu'à l'état d'une seule bande, cette distinction n'est pas toujours possible. Si, par exemple, les éléments qui sont en contact avec la portion interne du bois ressemblent par tous leurs caractères à ceux qui aroisinent la surface de la nervure, on ne pourra établir de démarcation en moelle et massif périphérique; on pourra alors faire rentrer tout ce tissu dans le groupe externe. Cependant, si des cellules d'aspect particulier différentes du ligneux, du liber et du parenchyme environnant, siègent dans la concavité supérieure de l'are, on pourra réserver les termes de moelle à l'ensemble de ces éléments.

a. Massif périphérique. - Un certain nombre de plantes, comme la plupart des Borraginées, ont autour du système libéro-ligneus de leurs nervures foliaires un parenchyme dont les collules possìdent toutes la même forme, la même structure; nous pouruns donner I'épithète d'homogèné à un massif ainsi constitué. Ordinairement, les cellules d'un tel parenchyme sont assez grandes, polyédriques, dépourvues de chlorophylle ou ne renferment qu'une très faible quantité de cette substance. Leur paroi cellulaire est mince ou peu épaissie arec ou sans ponctuations. Elles laissent entre elles de nombreux méats. Les feuilles des plantes aquatiques, comme celles de Menyanthe (Monyanthes tirfoliata. L.), sont munies d'un parenchyme offrant un grand nombre d'espaces lacuneux. 
Toutes les nervures sont loin de présenter cette uniformité. Soit que les éléments diffèrent sur les deux faces de la feuille, soit que plusieurs espèces de cellules réunies en tissu se rencontrent sur la même face foliaire, le massif est hélérogène. Nous n'avons point l'intention d'étudier les mille dispositions qu'affecte la masse parenchymateuse. Nous choisirons seulement parmi les nombreux cas de variations, quelques exemples saillants, types du groupe des tissus externes.

Prenons d'abord la feuille du Buis (Buxas scmpervirens. L). Si nous faisons sur la nervure et à quelques millimètres de la base de la feuille une coupe mince transversale et si nous observons ensuite cette tranche au microscope, notre examen nous fera distinguer les particularités suivantes :

Directement au-dessous de l'épiderme supérieur s'étendent quelques assises de cellules vertes, cylindriques, perpendiculaires à la surface de l'organe. Au-dessous du liber et entre ce dernier et l'épiderme inférieur, les cellules sont arrondies, moins colorées et plus courtes. Le parenchyme inférieur et le parenchyme supérieur diffèren $t$ ainsi l'un de l'autre, mais chacun de ces tissus est uniforme.

Portons maintenant notre attention sur la feuille de l'Epine rinette (Berberis vulgaris. L.); ici, il y a hétérogénéitẻ de l'amas situé à la face inférieure des faisceaux.

En effet, tout contre ces demiers existent plusieurs couches de cellules dont la paroi est lignifiée, et au-dessous de ce tissu est placé un parenchyme puissant dont les éléments larges sont limités par une membrane de cellulose. Nous pourrons désigner cet ensemble sous le nom de massif hétérogène ì tissus superposés, on plus simplement hétérogène superposé, pour le distinguer d'un autre cas fourni par quelques Ericinées, la Gaultherie couchée (Gaultheria mºcumbens. L.), par exemple.

La portion parenchymatense qui s'étend entre le liber et l'épiderme inférieur de la nervure, est aussi composée de deux sortes d'élíments; les uns, petits, ont leurs membranes assez épaissies et ponctuées, les autres, plus grands, possèdent une enveloppe mince. Ces 
cellules, au lien de former deux gronpes bien distincts, sorat miduen les unes aux autres. Wous pourrons consacred it cet assemblage

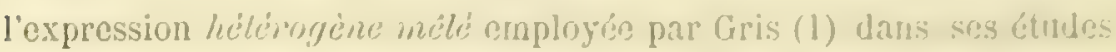
sur la moelle des plantes ligneuses.

Yous pourrious multiplier les exemples, mais les diverses dispusitions que l'on peut observer rentrent dans les grands tyjes que nums venous de signaler et que nous résumons dans lo tableau suivant :

A. Masse parenchymateuse homogine aux denx faces. - Borraginées.

B. Masse parenchymateuse hétérogine.

1. Homogène sur une mêrne face. - lßuis.

2. Hétérogène superposéc. - Eipine Vinetto.

3. Hétérogène mêlée. - Gaultherie.

b. Moelle. - La moelle est habituellement composéc de cullules polyédriques dont la membrane peu épaisse est lisse ou présente des ponctuations. Il y a presque toujonrs entre elles des méats plus ou moins considérables.

Généralement, le volume de ces éléments est d'autant p'us considérable qu'ils sont plus éloignés de la portion ligneuse des faisceaux. Dans certaines espèces, comme le Noyer, les membranes cellulaires sont lignifiées. Enfin, certaines moelles (Saponaire, Srquanaria ollicinalis. L.) sont constituées par des éléments dont l'aspect rappelle celui du collenchyme, c'est la molle enllenchymalezes.

\section{Organiles isole's ou groupess arcirlentels.}

Fous venons de passer rapidement en rerue les tissus qui forment le fond principal de Ia nervure; il nous reste à dire quelques mots des éléments que l'on peut rencontrer accidentellement. Comme le mésophylle, la nervure est capable d'offrir au milieu de ses divers tissus: $1^{\circ}$ des cellules à cristaux; $2^{\circ}$ des glandes internes; $3^{\circ}$ des élé-

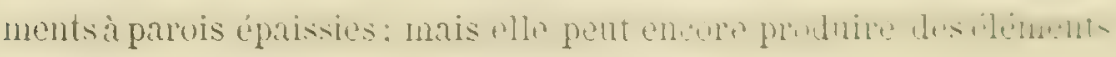
spéciaux qui ne se remarquent point ailleurs.

(1) A. Gris. De la moelle liqneuse. Ann. sc. nat. je série, t. 11, 187.. 
Nous voulons parler des canaux sécréteurs et des vaisseaux laticifères.

a. Canaux séciéteurs (1). - Les canaux sécréteurs sont des cavités allongées sans paroi propre, entourées de cellules distinctes de celles qui les environnent par la forme et le contenu. Ces cellules, dites celbules de bordure, ont la propriété de sécréter des produits habituellement oléo-résineux qui viennent se déverser dans le canal qu'elles limitent.

Les Térébinthacées, les Ombellifères, plusieurs Synanthérées, les Araliacées et les Conifères sont particulièrement riches en ces appareils.

Le nombre des cellules de bordure et de leurs assises est extrêmement variable. Tantòt ce nombre est fixe comme dans le Tussilage (Tussilago farfara. L.) oi les canaux sont bordés par quatre celIules sécrétrices (pl. VII, fig. V, c), tantòt il est changeant dans la même feuille. Dans certaines Térébinthacées les cellules de bordure atteignent un chiffre très élevé (pl. VII, fig. III, a, a', a').

La situation et le nombre des canaux présentent souvent un caractère de fixité tel que ces derniers peuvent à la rigueur servir à distinguer soit certaines familles, soit les espèces d'un même groupe. Ainsi les Térébinthacées ont des canaux résineux au milieu du liber de leurs faisceaux (Sumac vénéneux (Rhus toxicodendron. L.), pl. VII, fig. III, a, $\left.\mathfrak{a}^{\prime}, \mathrm{a}^{\prime \prime}\right)$.

Les Ombellifères, Synanthérées, Araliacées en présentent seulement dans le système fondamental. On en trouve dans certaines fenilles à la face ventrale et dorsale des cordons libéro-ligneux, soit contre les faisceaux, soit éloignés de ceux-ci (Ache odorante (Apium graveolons. L.), pl. VI, fig. III, c, g). D'autres n'en possèdent qu'à la partie dorsale (Amica monlana. L.).

(1) Trécul. Des vaisseaux propres chez les Ombellifères. Ann. sc. nat., 5e série, t. V, 186t?.

Trícul. Des vaisseanx propres chez les Aroïlées. C. R. A. Sciences, t. $61,1865$.

'Trécul. Des vaisseaux propres chez les Araliacées. Ann. sc. 1ـat., 50 série, t. VII, 1867.

Van Tieghem. Mémoire sur les canaux sécréteurs des plantes. Ann. sc., nat, כ́ série, t. 16, 187:. 
Laticifines (1). - On appelle ainsi des bléments on des tubeserioniralement allongés et sonvent ramitiés qui possident une parri joropro: et renferment dans leur caritó un suc particulier dosience sons le non de lielex.

Le latex est un liquide qui tient en suspension des grobules de diverse nature en partie composés de matieres grasses. Cro sue á une coloration qui varie d'une plante à l'autre. Incolore dans certaines espèces (Pervenche, Vinere minno. L.), blanc de lait dans plısieurs Chicoracées (Pissenlit, Tararneum dens lenmis. Jup. Th.), il est coloré soit en jaune, soit en ronge dans d'antres régétanx. Le latex coagule sous l'infuence de la potasse, aussi met-on it profit cette propriété pour constater co suc, ainsi que les éléments dans lesquels il se trouve renfermé.

Les laticifères sont tantòt de longues cellules amincies en pointe aux deux extrémités, simples ou émettant des branches ylus ou noins nombreuses; tantòt des tubes allongés résultant de la fusion de collules primitivement placées bout ì bout et séparées les unes des autres par des cloisons transversales. Les premicres ont été nommées par de Bary laliciféres non articulés, les secondes lalieiferres arliculés.

Les laticifères non articulés s'observent dans les Apocynées et les Asclépiadées. Ils se présentent dans la Perrenche (Vineel) sous l'aspect de fibres simples à paroi mince. Les Chicoracees offrent au contraire des laticifères articulés.

La localisation de ces réservoirs est sujette it de nombreuses variations.

Ceux des feuilles de Perrenche (Vinca minor. L.), de Chicorée (Cichoriun intybus. L.), de Pissenlit (Truaxacum dens leonis. Des.) sont situés dans le système fondamental et adossés à la partic péripherique du liber. On les rencontre au contraire dans le tissulibérien, dans la nervure des feuilles du Lobetia inflala.

(1) 'Tricul. Diver's mémoires sur les Liliciferes en complutis-rendus de litciatdemic des sciences, t. ILY, LI, LX, LXI, LXII, LIV, LIII.

David. Uber die Milchzellen der Euphorbiaceen. Breslan, 187:. 


\section{() H I ITRE II}

\section{TECHNIQUE.}

Les feuilles que l'on trouve dans les drogueries ou dans les pharmacies sont généralement desséchées. A cet état, elles sont impropres à l'examen histologique. Essaie-t-on d'en faire, à l'aide d'un rasoir tranchant, des sections minces destinées à l'observation microscopique, les feuilles se brisent en parcelles très-menues qui ne sont d'aucune utilité pour l'étude. Il est donc nécessaire de faire subir à ces organes des manipulations préliminaires qui permettent de pratiquer facilement des coupes régulières d'une assez grande étendue, et rendent aux éléments anatomiques un aspect semblable à celui qu'ils possédaient à l'état frais.

Voici les procédés auxquels nous avons eu recours :

On place dans une capsule en porcelaine ou dans tout autre vase pouvant supporter la chaleur, de l'eau distillée et quelques fonilles entières ou plusicurs fragments d'organes. On fait bouillir le mélange. Quand les ot jets sont suffisamment ramollis, on retire le récipient du feu et on laisse refroidir. Une portion des fragments peut être employée à la confection de coupes fines avec un rasoir, et par des procédés que nous indiquerons un peu plus loin. Lorsqu'on a obtenu une assez grande quantité de tranches délicates, on en observe une ou deux dans l'eau, au microscope. L'examen superficiel a-t-il fait remarqué que les tissus ont acquis leur forme primitive? on pourra alors les étudier d'une façon plus approfondie, et faire agir sur eux les divers réactifs. Les éléments anatomiques sont-ils encore rétractés? il est alors de toute nécessité de procéder à une seconde opération.

On doit aussi essayer de détacher quelques lambeaux d'épiderme d'antres fragments, en s'aidant d'une pince fine ef d'un petit scalpel. 


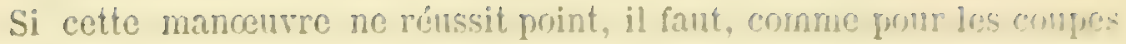
imparfaites, user d'une antro méthode.

Fous nous sommes selvi avec avantage d"une solution failsle de potasse caustique.

A cet effet, un certain nombre de tranches sont mises dans de l'erils distillée additionnée de quelques gouttes d'une solution de protasse à $1 / 10$, et contenue dans une petite capsule en porcelaine. Un agit de même à l'égard d'une feuille enticire ou de débris. Les apparcils sont chauffés. Gràce à celte opération, les cellules font disparaitre les plis produits par la dessication, et il est facile d'enlever des portions d'épiderme. Nous avons pu, par ce moyen, rendre aux tissus de feuilles conservées depuis longtemps en herbier, une forme senblable à l'aspect qu'elles revêtaient à l'état frais.

La potasse n'altère point la forme des cristaux qui siegrent dans les feuilles; elle a de plus l'avantage de produire la coagulation du latex. congulation qui permet de constater la présence de raisscaux ou cellules qui renferment ce suc.

Pour obtenir facilement de nombreuses coupes minces, on intercale des feuilles ou des fragments entre deux morceaux de moelle de sureau. On superpose plusieurs feuilles ou débris pour aroir une assez grande épaisseur.

Comme parmi les nerrures, le cordon médian a seul fait l'objet de notre étude, puisque nous avons fait nos descriptious d'après des coupes transversales pratiquées à une petite distance de la base de la feuille; on doit, pour ne point tomber dans des erreurs de diagnose, disposer des feuilles ou des fragments choisis conrenablement, de facon à produire des sections à la hauteur désirée.

La moelle de sureau peut être tenue entre les doigts ou introduite dans un microtome. Celui que nous employons est du modèle Ranvier. Cet instrument permet de faire un grand nombre de coupes minces et très étendues dans un espace de temps très court.

Les coupes obtenues sont alors placées dans de l'eau que renfirme un velre de montre, et peuvent être ensuite examiuées au microscope en suivant les méthodes indiquées en micrographie. 
Lorsqu'on veut se rendre exactement compte de la forme, du rapport des éléments anatomiques, il est nécessaire de fabriquer des sections dans le sens longitudinal de la feuille, mais ces moyens ne donnent point toujours des résultats convenables. On recourra alors utilement à la dissociation des éléments. On peut faire usage d'une solution concentrée de potasse que l'on porte à l'ébullition avec les parties que l'on désire observer. Ce procédé convient, principalement, à la recherch des Laticifères.

Il ne suffit pas, pour déterminer une feuille, d'examiner au microscope la forme, le rapport des cellules, des fibres, des vaissaux, il faut encore recomaitre les propriétés chimiques de la membrane et du contenu. L'emploi des réactifs est alors indispensable. Nous ne signalerons que les principaux; car nous n'arons pas eu pour but de rechercher dans les éléments anatomiques les produits si complexes, comme les alcaloïdes, dont la présence est actuellement difficile à constater à l'aide de substances de coloration.

La distinction des différentes feuilles employées en thérapeutique, ou falsifiant ces dernières, nécessite seulement l'usage de quelques liqueurs dont nous domnons ci-dessous la liste :

$1^{\circ}$ Solution d'iode ioduré; $2^{\circ}$ Chloroiodure de zinc; $3^{\circ}$ Solution ammoniacale de fuchsine; $4^{\circ}$ Acide acétique; $5^{\circ}$ Acide chlorhydrique.

La solution d'iode produit la coloration bleue de l'amidon; elle n'a aucune influence sur les membranes de cellulose, mais détermine la coloration brune de la cuticule et des parois lignifiées.

Le chloroiodure de zinc colore au contraire la cellulose en bleu ou en violet; la cuticule et le ligneux en brun.

Si l'on plonge des sections de feuilles dans une solution incolore de fuchsine dans l'eau ammoniacale, et qu'on les transporte au bout d'une minute dans de l'eau distillée, les portions cuticularisées ou lignifiées acquièrent une magnifique coloration violette qui ne se manifeste point sur les parties cellulosiques.

Quant aux acides acétique et chlorhydrique, ils servent à reconnaitre la nature des cristaux. Le premier est sans action sur l'oxa- 
$-11 ; \ldots$

Iate de chanx, mais dissont avec effervescence lo carbonate de chanx; le second, au contraire, produit la dissolution de ces denx substances chimiques.

Si l'on désire conserver en préparation des coupes de feuilles, on ponrra faire usage d'un mélange en parties égales, d'eau distillée et de glycérine. 


\section{CHAPITRE III}

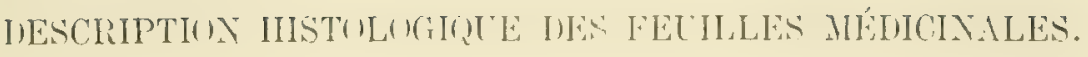

Nous nous sommes adressé aux feuilles que signalent le Codex francais et les remarquables traités de pharmacologie de M. G. Planchon (1) et de MM. A. Fliickiger et D. Hanbury (2). Nous avons adopté, dans nos descriptions, l'ordre des familles naturelles qui, clles-mêmes, sont classées suivant la méthode de A. de Jussieu. Les feuilles utilisées en médecine et les organes qui servent à les falsifier ou peuvent être facilement confondus avec elles, ont été décrits avec assez de détails.

Nous avons fait suivre la description étendue d'une feuille officinale, de la diagnose différentielle de cet organe et de ses substitutions, diagnose établie d'après les caractères histologiques les plus saillants.

Dans le cas où une famille végétale est représentée par plusieurs plantes dont les feuilles sont employées en thérapeutique, nous avons groupé dans un paragraphe qui suit l'indication de la famille, les principaux faits que nous a fournis l'analyse des diverses espèces et énuméré, toutes les fois qu'il y avait lieu, les caractères communs aux types médicinaux du même groupe.

Nous avons constaté que les espèces appartenant à chacune des grandes familles (Labićes, Borraginées, Solanées, Synanthérées, etc.) offrent dans leur structure plusieurs points de ressemblance. L'ensemble de tels caractères, propre à chaque groupe, mais différant d'un groupe à l'autre, peut servir à établir des distinctions entre les diverses familles.

(1) G. Planchon. Traité pratiçue de la détermination des drogues d'origine végétale. Paris, $18 \%$.

(2) A. Flückiger et D. IIanluury. Histoire des drogues d'origine végétale, traduit de l'anglais et annoté par. M. de Lanessan. 
Nous sommes loin de prétendre que cotte somme de dispositions histologiques, commune aux feuilles médicinales d'un môtur gronje. puisse se retrouver dans tous les types de cette fauillo. Il pent ru eflet arriver que quelques espoces, formes do passuge entre deux familles voisines, ne possident point quelques-uns do cos traits gínéraux. Nous n'accordons qu'une valeur limitéc ì ces caractíres. Lin appliquant ceux-ci a certaines familles assez riches en plantes officinales, nous supposons que ces groupes ne comprennent que les types sur lesquels ont porté nos recherches.

\section{GYMNOSPERMES.}

FAMILLE DES COXIFFRES.

Feuille de Génévrier sabine. - Sabine. - Juniperus Subina. I.

L’épiderme ne présente jamais qu'une assise de cellules. Si on enleve l'cpiderme de la face inférieure de la feuille et quon l'examine snus lo microscope, on lui tronvera la structure suivante. Les cellules sont allongées dans le sens de la longueur de lorgane; elles ont la tome de rectangles ou de trapèzes. Leurs parois sont épalissies ct munies de ponctuations qui manquent. il est vrai, sur les parois parallites it la surlace du limbe foliaire. Les parois transrersales sont tantut horizontales, tantôt obliques. Cet épiderme est dépour'ru de stomates.

Lépiderme supérieur est beaucoup plus compliqué. On y distingue plusieurs régions très distinctes ver's la base du limbe et se caracterisant par la différence d'aspect des cellules, aussi bien que par la présence ou l'absence des stomates. La portion épiderminue qui occupe les parties latérales extrèmes de li feuille, forme de chaque cuté une bande cellulaire dont les éléments, trés longs, trés épaissis, et munis sur leurs parois de ponctuations, ressemblent it ceux qui constituent l'épiderme de la face inférieure. Chacune de ces bandelettes privées d'appareils stomatiques, touche une autre bande qui se reconnait par ses cellules très courtes, limitées par une membrane assez. mince finement ponctuée. C'est cette région qui othie des stomates. Ceux-ci sont disposés assez irrẻgulièrement: leur ouverture, dirigée dans le sens de la longueur du limbe, est bordée de deux cellules réniformes. Si l'on pratique des sections transversales très fines d'une feuille, les stomates se montreront composés de la façon suivante: les réritables cellules de bordure sont surplombées par des éléments qui laissent entre eux. au-dessus du canal stomatique, un espace, une antichambre. Cette disposition, on le sait, est particulière aux Conifëres.

Enfin, au milieu de la surface foliaire, et entre les deux rẻgions stomatiques, s'étend une bande dont la largeur est assez faible, et manque des petits appareils que nous renons de décrire sommairement. Les cellules, beaucoup plus longues que celles de la portion précédente. 
ont une enveloppe bien moins épaisse que la membrane des cellules épidermiques latérales.

Les cellules des deux épidermes sont recouvertes extérieurement d'une cuticule très épaisse.

L'épiderme enveloppe un ensemble d'éléments d'autant plus développé en épaisseur qu'il aroisine le plan médian de la feuille.

Une ou deux assises continues d'hypoderme (pl. VIII, fig. III, b) sont en contact arec l'épiderme inférieur. Les éléments de ce tissu prẻsentent des parois très épaissies et ont la forme de longs fuseaux terminés en pointe mousse ì leurs extrémités. De scmblables fibres hypodermiques n'existent que sur les portions latérales de la face supérieure de la feuille.

La masse fondamentale la plus importante est constituée par des cellules dont la cavité renferme de la chlorophylle $(b, g)$. Les cellules de ce parenchyme convergent de la périphérie ver's le milieu de l'organe; leur's parois sont assez minces et leur aspect est assez variable. 'Tantôt ces cellules sont ellipsoïdales, tantott elles sont cylindriques. Les plus grandes occupent habituellement la région médiane de la face supérieure. C'est dans ce mésophylle et un peu an-dessus de l'insertion foliaire que se rencontre une grosse glande $(p)$. Celle-ci se montre à la face inférieure de l'organe, elle touche d'une part l'hypoderme périphé. rique et le système fasciculaire central; elle est formée de plusieurs cellules sècrétant une substance oléo-résineuse. Lorsque la glande est adulte, elle est composée d'un assez grand réservoir centrâl où sont accumulés des globules d'oléorésine, et de quelques assises périphériques de cellules rectangulaires, ì membrane assez épaissie.

Un faisceau libéro-ligneux peu volumineux chemine au centre de l'organe.

Ce cordon est constitué par du liber et par du bois.

Le liber $\mathcal{C}$, situé à la face inférieure du faisceau, est formé d'un amas de fibres assez grosses, dont les parois assez minces sont cellulosiques. Ce massif occupe la partie inférieure du liber, tandis que sa portion supérieure est composée de cellules très petites disposées en séries r’égulières inféro-supérieures.

De fines trachées et des fibres dont la membrane est munie de ponctuations aréolées, ou d'épaississements réticulés (pl. VIII, fig. IV, a) entrent dans la constitution du bois qui est très peu développé.

A chaque flane du ligneux est adossé un îlot de cellules cylindriques assez grandes pourvues sur leur paroi de ponctuations aréolées (Tissu aréolé), pl. VIII, fig. IV, b, c.

Feuille de Génévrier de Virginie. - Juniperus Virginiana. L.

On peut confondre les feuilles du Génévrier de Virginie avec celles de Sabine.

Nous allons donner les caractères qui permettent de distinguer ces deux espèces.

Si l'on pratique une section transversale de la feuille de Sabine, section passant par l'appareil glandulaire, et qu'on l'examine au microscope, on constatera que les cellules situées entre le faisceau et la portion médiane de l'épiderme supéricur, sont allongées perpendiculairement ì la surface et sont limitées par une membrane mince. 
Une section fitite an meme nivenu dans nue fenillo de founewiog do

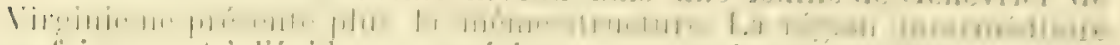

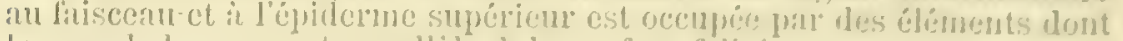

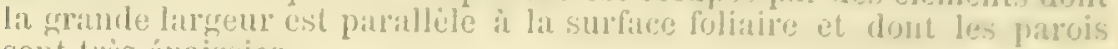
sont tres épaissies.

Le liber de cette espice oflie en ontre iles fibres dont la cavite, pretile. est enveloppée par une menbrane tris épinissie.

\section{ANGIOSPERMES MONOCOTYLIDONES.}

FAMILLE DES ORCIMTEES.

Feuille de Faham, - Angrecum frograns. Dup. Thouars.

I. Epiderme. - Lépiderme est constitué par une couche de collules incolores, tabulaires, dont les parois sont filblement épaissies. Lenr

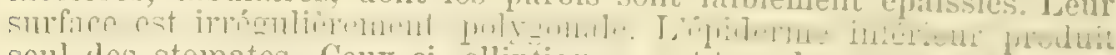
seul des stomates. Cenx-ci, elliptiques, ont tous leur criand diumetre dirigé dans le sens de la longueur de la feuille. L'épiderme est reconvert d'une cuticule épaisse.

11. Mésophylle. - Contre les deux épidermes sappuie une assise le collules incolores à membiane un peu épaissio. Ces cellules se montrent polygronales sur une conpe transrersale, rectanoulaties ou carrées sü une coupe longitudinale. Dans leur milien sont disséminés de lonrs bé. ments dont les extrumités se terminent en pointe. Les parois épaissies de cos éléments sont cellulosiques on lignifiées: leur carité est consi-

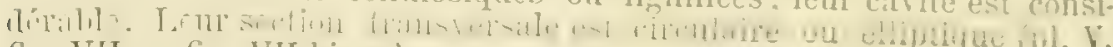
fig. Vil, a, fier. VII bis, a)

Entre les assises incolores, siẻre du parenchyme rert uniforme. Les éléments nombreux qui lo constituent sont ellipsoüdaux, oroüles ou

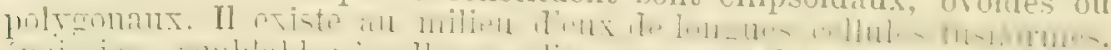

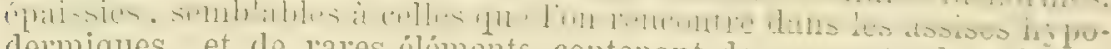
dermiques, et de rares éléments contenant des paquets de cristaur aciculares (raphides) d'oxalate de chaur. Les derniers sont difficiles it yoir, parce que sous l'inlluence de l'eau il s'échappent des cellules.

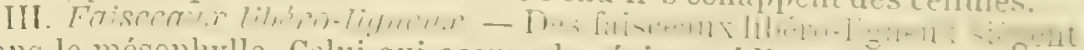
dans le mésophyllo. Celui qui occupe la région médiane de la fenille est

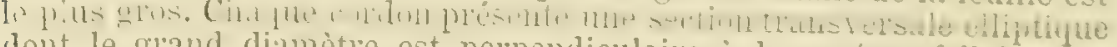
dont le grand diamètre est perpendiculaire à la surface folizile. On

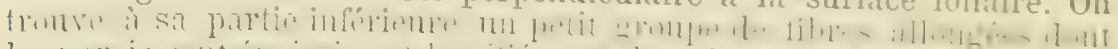

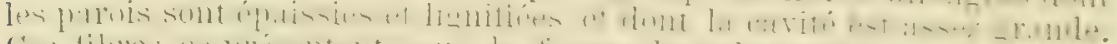

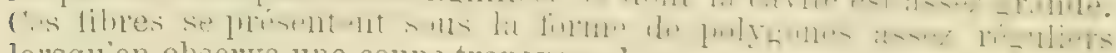
lorsquon observe une coupe transtersale.

du dessus on rencontre un amis de liber celluleux, qui est ì sa face

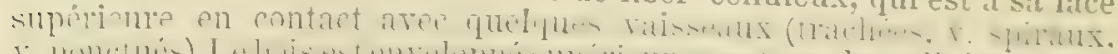

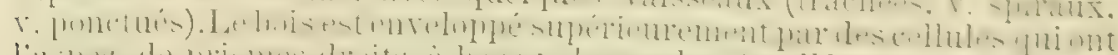

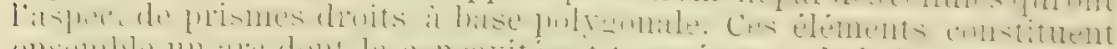

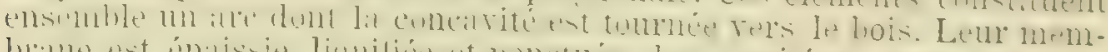

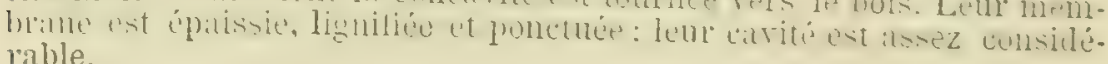
rable. 


\title{
ANGIOSPERIES DICOTYLÉDONES
}

\author{
FAMTLLE DES PIPÉRITÉES.
}

Feuilles de Matico. - Artanthe elongata. Mir.

I. Lame interneriale. a. Eliderme. - L'épiderme for'me une seule couche de cellules aplaties, polygonales superficiellement. Pourvu de stomates à sa face inférieure senlement, il est hórissé de longs poils, composés d'une seule série de cellules et surmontant une petite saillie épidermique pluricellulaire. Les cellules qui cntrent dans la constitution de chacun de ces poils sont beaucoup plus longues que larges et les basilaires surtout présentent la particularité d'ètre plus larges à leur base et à leur sommet qu'à leur région médiane. La cellule supérieure, assez longue, est conique.

b. Mésophylle. - Sous l'épiderme supérieur on rencontre une ou deux assises de cellules incolores rectangulaires ne présentant aucun méat; et, au-dessous de ces assises, de deux ì quatre couches de petites cellules vertes de quatre a cinq fois plus longues que larges, pressées les unes contre les autres, cylindriques et perpendiculaires à la surface de la feuille. Contre l'épiderme inférieur est adossé du parenchyme vert moins dẻveloppé que le précédent. Il est composé de petites cellules un pen rameuses, arrondies ou allongées en différents sens, lorsqu’on examine la coupe transversale de la feville. Le Mésophylle est dépourvu de cellules cristalligènes, mais renferme des glandes uni-cellulaires contenant une huile essentielle jaunatre. Ces dernières, sphériques ou elliptiques, à grand diametre perpendiculaire à la surface épidermique, sont surtout situées dans le parenchyme supérieur, non loin de l'épiderme.

II. Nervure. - Au-dessous d'un épiderme poilu à cellules petites, rectangulaires, siègent ordinairement deux assises de cellules ar'ondies, rectangulaires en coupe transversale. Contre ces couches existent des îlots de cellules collenchymateuses très épaissies et très longues (pl. VII, fig. II, b). Ces îlots sont séparés les unes des autres par des cellules qui forment aussi en dedans d'eux un massif puissant. Ces dernières ont leurs membanes épaissies et présentant des ponctuations; elles sont irrégulièrement polygonales sur une coupe transtersale, carrées ou rectangulaires sur une coupe lon ritudinale. Quelques-uns de ces éléments renferment de la chlorophylle, mais presque tous contiennent de nombreux cristaux isolés qui ont pour la plupart la forme de petites aiguilles ou de petits prismes. Des cellules lignifiées et très épaisses $\mathrm{g}, \mathrm{g}^{\prime}, \mathrm{g}^{\prime \prime}$, ainsi que des glandes unicellulaires existent dans ce parenchyme. Ces sclérites sont polygonaux ou arrondis en coupe transversale, carrés en coupe longitudinale. Ils sont munis sur leurs parois de nombreuses ponctuations, la plupart allongées radialement. Les glandes sont ellipsoïdales à grand diamètre longitudinal. Le système fibrovasculaire est représenté par des îlots $(e, f)$, en nombre variable, disposés en un arc concave supérieurement et séparés les uns des autres par plusieurs rangs de cellules analogues à celles qui composent le tissu fondamental. Les îlots présentent chacun une section transversale ordinairement triangulaire; ils sont situés vis-ì-vis les cordons collen- 


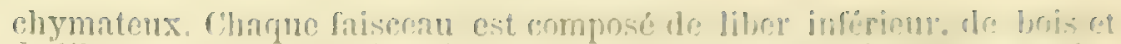

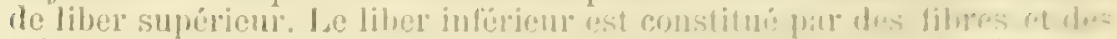

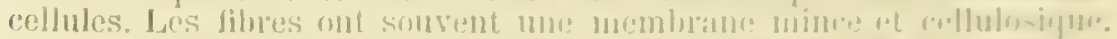

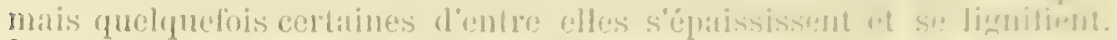

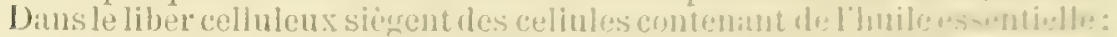

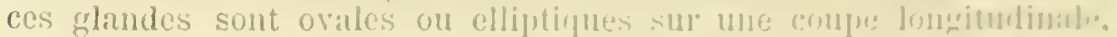

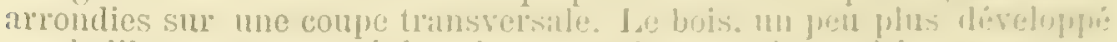

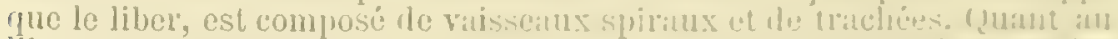

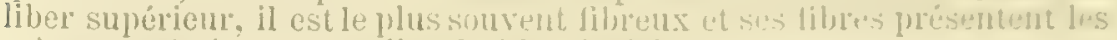
memes variations gue celles du liber inlerieur.

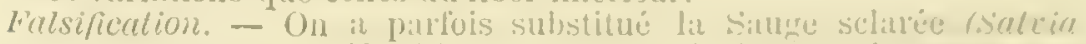
sclarea. L.) au matico. Voici les curactires principax qui peranctuon de les distinguer.

Dans la Sauge sclarée (Salzia scle'éa. L.) il n'y it jumais lle cellule. incolores au-dessous de lëpiderme supérieur", ni de glandes unicellulaires dans le mésophylle. On trouve atu contraire des glandes cexter. nes. Il n'existe point de fuiscenux séprứs de collenchine dans lit nervure. Il n'y a pas non plus de sclérites, le galandes, ni de cellules di cristaux dins le parenchyme neural.

\section{Feuille de Serronin Jubriondi Gaud.}

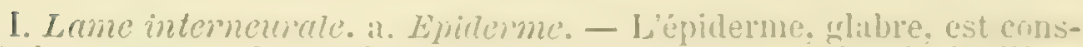
titué par une seule conche de collules tabulaires it surlince irréculiere.

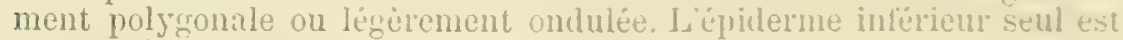
pourvu de stomates.

b. Mésophylle. - Au-lessus de lépiderne inférient uxiste une on deux couches de mantes cellules incolures arlondies ou rectanmulares. Entre ces assises et lépiderme supúrieur so troube du frarouchrine rert. en général un peu moins développé que la misse totale de lépliderme et de l'hypoderme. Superieurement, le parenchrme lome une assise de tros petites cellules cylindriques, it peine deur fois plus longues que larres. Inléreurement, il est constitue par plusieurs couches de cellules arrondies.

Les cellules cristalliwenes sont absentes: mats il existe des alamdes unicellulailes sphérques, sécrótant une huile essentielle jauraitre. Ces dernières sont placées dans l'hypoderme ou au milieu du mésophrlle.

II. Nerzuré- En dedans d"un épidelme à cellules un peu épaissies. rectangulaires, siège un massif de cellules très longucs et trés épaissies constituant un collenchyme. En dedans du collenchyme infírieur se trouve une ou deux couches de loners éléments épaissis, limnifiés, terminés en pointe íl leur extrémité. Entre ces tibres et le collenchyme supélieur est une masse d'úléments polyonatr e: section transversale. carés ou rectangulailes à peine deux fois plus longrs que larges dans le sens longitudinal. Ils laissent entre eux de faibles méts. ()ueloues-uns renterment de lia chlorophylle. d'autres, au contraire, ont un contenu tres granuleux et cristallin. On trouve dins ce parenchyme des cellules ¿ membrane trés épaissie, ponctuée et ligniliée. arondie ou irrégulièrement polyonale en section transversale, presque carre dans le sens longitudinal.

Le système fibro-vasculaire est leprésenté par plusieurs cordons libélo-ligneux disposés eu arc et séparés les uns des autres par des cellules semblables i celles du paremchyme fondamental. Chaque cordon 
est oval (coupe transversale), allongé perpendiculairement ả l'épiderme. Il est composé en allant du bas ver's le haut:

1') De libel constitué inférieurement par un amas continu de fibres épaissies, lignifiées, ponctuées, à carité plus ou moins grandes; supérieurement par des cellules libériennes au milieu desquelles sont plongées des glandes unicellulaires dont le contenu est oléo-résineux. Ces dernières sont généralement allongées suivant la nervure:

¿e De bois formé de vaisseaux rayés, spiratux, trachéens:

$3^{\circ}$ D'un massif de fibres ligníiées semblables à celles du liber inférieur.

\section{Poivre réticulé. - Piper reticulatum. L.}

1. Lame interncurale. - Le mésophylle vert est, à la face inférieure comme ì la face supérienre, situé entre deux couches de cellules incolores et aplaties. Les cellules de l'assise superficielle présentent un contour polygonal. Des stomates se reneontrent à la face inférieure. Les deux épidermes produisent des glandes unicellulaires ayant la forme d'un auf dont la petite extrémité est tournée en haut, glindes supportées pal un pédicelle à une seule cellule

Au-dessous des couches incolores supéricures siège une assise de mrandes cellules cylindriques vertes, perpendiculaires à la surface de Ir feuille et de deux it trois fois plus longues que larges. Ce tissu est en contact avec une lame composée de trois a quatre couches parallèles de cellules qui, sur unc coupe trinsversale, sont allongées parallèlement ¿̇ l'épiderme. Elles laissent entre elles des méats nombreux, mais très petits.

De grosses glandes unicellularies sphériques se trouvent dans ce parenchyme, au voisinage du tissu en palissade. Elles sont remplies de gouttelettes d'huile essentielle jaunhtre.

II. Nervure. - Contre l'épiderme inférieur siège un arc continu de collenchyme au-dessus duquel on voit un amas de parenchyme dont les éléments incolores, sont prismatiques i base polygonale et laissent entre eux de petits méats. Des glandes unicellulaires se rencontrent dans ce parenchyme.

Au-dessous de l'épiderme supérieur existent deux ou trois couches de grandes cellules incolores, qui touchent ì quelques assises de cellules contenant un petit nombre de grains de chlorophylle. Entre ce tissu et le parenchyme inférieur se trouve un cordon libéro-ligneux composé inférieurement d'un arc de liber dont les éléments ont leur paroi cellulosique. Des cellules sphériques remplies d'huile essentielle se remar'yuent dans le liber.

Au-dessus du liber est le bois constitué par des vaisseaux ponctués et spiraux et des trachées. Enfin le bois est en contact supérieurement avec des éléments semblables à ceux qui composent le liber inférieur.

FAMILI.E DES JUGLANDEES.

Feuille de Noyer. - Juglans regia. L.

I. Lame interncurale. a. Eniderme. - L'épiderme, mnuni de stomates ì la face inférieure seulement, est formé d'une seule couche de 
petites cellules minces, tris aplaties, iménliument polyemales it la

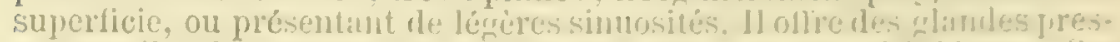

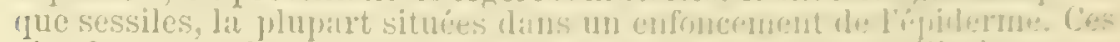

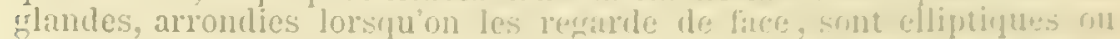

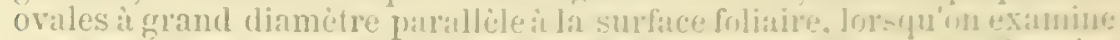
une coupe transtersale. La plupart sont pluricellulatres at formues d'un rang de plusienrs cellules séparées par des cloisnns verticales.

b. Mésophylle. - Les cellules supéricures du unémoliyllo sont cylindriques, pressées les unes contre les atutres, de quatre at six fois flus longues que laroes et disposées en palissade. Jilles constituent deux couches. Dans ce parenchyne el surtout dans lá conche superficitles

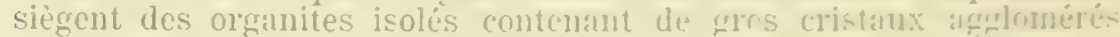
d'oxalate de chaux. A la face inférieme, existent les cellule rameuses formant une lame d'énaisseur éfale it celle du barenchyme superieur. En coupe transtersale. ces éléments pésentent deux ou trois petites branches; ils sont dirigés en tous sens et laissent mine cux de mornbreux miats. Vus de face. ils ont ordinairement le mime aspect, el les espaces intermédiaires sont assez larges et de forme jolironale. Ce tissu rameux n’ofíe généralement point de cellules cristalligenes.

II. Nerurre. - Lépiderme, forné de petites cellules rectangulaires: domne maissance à des glandes et it des poils pluricellulaires composés dun seul rang de cellules. In dedans de l'épiderme sierrent deux ou trois couches de cellules à membrane épaissic et jonctuée : elles ont la forme d'un loug prisme ì base polygonale. A la partie interne de ces couches se troure un amas de cellules beaucouj plus larres et moins épaissies; quelques-unes contiennent des agromérations cristallines. C'est dans ce parenchyme qu'est placé un corilon composé de deux crois. sants libéro-ligneux tournant lenr bois l'un rers l'autre et súparés par des cellules nélullaires. Le liber est composé ì sa surface de fibres épaissies, ponctuées et lignifiées, formant un arc continu. En ledans d'elles, existent des cellules libériemnes constitunnt des rroupes atssez dételoppés, séparés entre eux par une on deux langées radiales de cellules assez épaissies. Certaines cellules contiemnent des cristaux. Le bois, un peu moins déreloppé que le liber, est formé de plusieurs lames vasculaires (fibres-vaisseaux ponctués, rayés, spiraux-trachées) assez espacées; entre elles on remarque des ravons médullaires à $2-4$ rangs de cellules épaissies, ponctuées et lignifiées. La moelle, située entre l’arc supérieur, est un amas de cellules, épaissies, ponctuées. ne laissant entre elles aucun méat. Ces cellules ont la forme de prismes à base polveronale. Celles qui sont roisines des trachees sont longues, petites et lipnifiées; tandis que les plus éloignées sont cellulosiques, plus larges, mais ont une hauteur moindre. On rencontre dans quelques-unes des cristaux d'oxalate de chaux.

FAMILLE DES MONIMIACÉES.

Boldo (1). - Preumus boldus. Molin.

I. Lame interncurale. a. Eniderme. - Lépiderme forme une seule

(1) A, Verne. Etudes sur le Boldo. - Thèse de l'Ecole supérieure de Pharmicie de Paris, $18 \tilde{T}^{4}$. 
assise de cellules très aplaties et i surface polygonale, cellules dont la membrane est très épaisse, ponctuẻe. L'épiderme inférieur possède seul des stomates; ainsi que le supérieur, il produit des poils sessiles en rosette (pl. IV, fig. I). Cette rosette, formée d'un nombre de branches variable, naît d'un petit groupe cellulaire enchâssé dans l'épiderme et composé en général d'autant de cellules qu'il y a de branches.

b. Mésophylle. - Au dessus de l'épiderme supérieur $e$, est adossée une couche $d$ de rrandes cellules incolores, très épaissies, et de forme ì peu près rectangulaire lorsqu'on examine une coupe transversale. Entre cette assise et lépiderme inférieur se trouve du parenchyme coloré par des grtains de chlorophylle. A la fice supéricure, les cellules sont cylindriques, pressées les unes contre les autres et allongées perpendiculairement it la surface de Ia feuille. Elles forment une ou deux couches (c).

Au-dessus de ce parenchyme en palissade viennent des assises paralIeles de cellules rameuses $b$ qui, sur une coupe transversale, ont le plus souvent lâ forme d'un rectangle à grand côté parallèle ì la surface.

Le parenchyme coloré, surtout l'inférieur qui est plus déreloppé quf le supérieur, ne produit point de cellules cristalligènes, mais de grosses cellules arrondies $a$ dont la cavité est remplie par une huile essentielle de coulemr jaune.

II. Marge. - Contre l'épiderme marginal est adossé un cordon assez puissant de fibres polygonales en section transversale. Ces fibres présentent un épaississement très considérable qui diminue beaucoup la cavité interne.

III. Nervare. - L'épillerme, beaucoup plus poilu que celui de lia lame, est constitué par des cellules épaissies, rectangulaires superficiellement. En dedans de lui existe un amas de cellules a membrane épaissie, ponctuée et ì cavité considérable. Ces éléments, plus nombreux ¿ la face inférieure, ont la forme d'un prisme droit à base polygonale, trois a six fois plus haut que large.

En dedans de l'hypoderme inférieur existent quelques couches de cellules à membiane plus mince, laissant entre elles de faibles méats. Elles ont à peu près la même forme que celles de l'hypoderme supérieur. Dians ce tissu se trouvent des glandes unicellulaires. Quant à l'hypoderme supérieur, il touche par sa partie interne, sur la ligne médiane, un groupe fibreux appartenant au faiscean libéro-ligneux, et latéralement du parenchyme vert qui fait suite ì celui de la lame.

Le cordon libéro-ligneux est composé en allant de bas en haut: $1^{\circ}$ de liber inférieur; $2^{0}$ de bois, tous deux en forme d'al' à concavité supérieure; $3^{\circ}$ d'un amas fibreux libérien situé dans la concavité que produit le bois.

Le liber est constitué inférieurement par des fibres épaisses, lignifiées, ponctuces, formant un arc continu; supérieurement par des cellules libériennes au milieu desquelles n'existent point de glandes unicellulaires.

Le bois, plus développé que le liber, est formé de bandes l'ayonnantes vasculaires, peu éloignées les unes des autres et séparées par des rayons à une ou deux rangées radiales de cellules épaisses, aplaties.

Le liber interne ou supérieur est fibreux, et les fibres ont la même forme que dans le liber externe. Souvent on remarque au milieu de cet amas prosenchymateux de petits groupes libéro-ligneux, tournant leur liber vers lépiderme supérieur. 
FAMILLE DES URTICERS.

Feuille de Parictairo ofneinale. - Parictarin offeinalis $\mathbb{I}$.

I. Lame intemeurale. a. Ejuderme.-- Une conche cellulare ronstitu: les deux épidermes. Lépiderme inférienr prísente des collules drun lo:

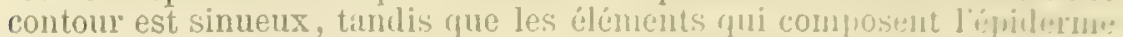
supérieur ont leur surface polygonale sans ondulations. La premier ext pourvu seul de stomates; mais tous les deux sont munis de prils molinaires et de poils glanduleux. Les poils unicellulaires sont de denx sortes: les plus communs ont lia forme d'hamecons; les antres, plus rares, sont droits et coniques. La base de ces denx especes de jobls est enchissée dans l'épiderme et se rétrécit hrusquement au niveau le la surface foliaire. Leur's parois sont épaissies it tel joint que la carité cellulare disparait. Cette dernière noxiste r'ordinare qu'il lat base, ou elle forme un petit cunc. Les glandes sont spheririres; elles sont composées de deux ou quatre cellules placées sur le nüme plan et séparées par des cloisons verticales. Le pédicelle qui les pnite est uni ou bicelluIaire. Parmi les cellules qui entrent dans ln contitution de lépiderme supérieur, il y en a de plus considérables qui renfer'm térieur des corps spéciaux anxquels on a donné le nom de cystolithes (pl. IV, fig. VII, a). Ceux-ci se présentent sous la forme de milsse ylobuleuse, rattachée al lil paroi superficielle par un dilament court et délié. Si on les examine dans un milien neutre ou alcalin, ils oflient une surface mamelonnéc (pl. IV, fig. VII); mais, sous l'intluence de l'acide acétique, ces mamelons disparaissent avec efrervescence et il reste un globe qui porte des stries concentriques. Ce dernier, traité par du chloroiodure de zinc, bleuit, ce qui prouve qu'il est constitué par. de la cellulose.

b. Mésopthylle. - Au-dessous de l'épiderme supéricur existe une couche de grandes cellules cylindriques ou elliptiques pressées les unes contre les autres et perpendiculatres à la surface de lit feuille. Ces éléments. qui contiennent de la chlorophylle, sont de trois a cinq fois plus loners que larges. Ce tissu en palissade est en contict inférieurement avec du parenchyme rameux dont les éléments, allongés parallẻlement à l'épiderme (coupe transversale), sont très rameux. Le mésophylle tout entier renferme des cellules incolores dans lesquelles se trouvent des cristaux agglomérés d'oxalate de chiux.

II. Nervure. - Les cellules épidermiques sont allongées suivant l'are de la nervure. Les deux épidermes donnent naissance ì des poils glanduleux et it des poils ordinaires, seulement les poils droits et coniques, rares sur la lame. sont au contraire ici les plus abondants.

Contre l'épiderme siègent quelques assises de collenchy̌me. Entre le collenchyme supérienr et le collenchyme inférieur se troure une masse de grandes cellules incolores qui laissent cntre elles des méats. Ces éléments, dont les membranes sont assez minces, ont la forme te prismes droits it base polygonale. Dans ce parenchyme est placé un cordon libéro-ligneux; le liber est composé d'éléments cellulosiques disposés en files longitudinales (quelques cellules contiennent des cristiux agglomérés); le bois est formé de deux ou trois couches de vaisseaux (tracliées, vaisseaux spiraux). 
FAIILLE DES EUPHORBIACEES.

Fenille rle Buis toujours rert. - Buxus sempervincns. I.

I. Lame intemeurale. a. Epideme. - L'épiderme infériem est composé de cellules a membrane faiblement épaisse, de forme polygo. nale quand elles sont vues de face, rectangulaires en coupe transversale. Muni de gros stomates arrondis, il est recouvert d'une conche de cuticule dont l'épaisseur est double de celle de la cavité cellulaire.

L'épiderme supérieur, dépourvu de stomates, est semblable à l'inférieur.

b. Mésophylle. - Le Mésophylle totalement rert est hétérogène. Le supérieur est ordinairement composé de 3 couches de cellules allongées perpendiculairement ì la surface supérieure; elles sont rectangulaires et ont une longueur qui dépasse it peine trois fois lemr largeur. La inembrane qui enveloppe leur contenu chlorophyllien et huileux, est faiblement épaissie. Les inférieures se rapprochent de la forme arondie.

Le mésophylle inférieur, qui égale en énaisseur le supẻreur, a des éléments rameux dont le plus grand nombre est allongé dins le sens de la surface foliaire. lls laissent entre eux des méats polygonaux.

II. Marge. - Le parenchyme vert vient s"appuyer directement contre l'épiderme sans qu'il existe de couche de renfoncement. Cependant, ì quelque distance de l'épiderme marginal et dans le tissu chlorophyllien, cn remarque un petit massif arrondi de tissu fibreux.

III. Nervure. - Sans stomates aux deux faces, l'épiderme est rectangulaire, allongé suivant la longueur de la nervure.

Entre ces deux épidermos, on trouve du tissu renfermant de la chlorophylle (pl. VI, fig. I, h, b), au milieu duquel se trouve la masse libéroligneuse. Le parenchyme vert (h), situé directement au-dessolis de l'épiderme supérieur et faisant suite avec le parenchyme en palissade qui existe de chaque côté de la nervure, se compose de quatre à cinq couches de cellules orales ou polyédriques. Le parenchyme situé audessus de l'épiderme inférieur, un peu moins épais que le supérieur, a des cellules arrondies moins riches en chlorophylle, et laissant entre elles des méats (b). Quelques-unes incolores, situées près du cordon fibro-tasculaire et disposées en file rerticale, renferment des cristanx isolés d'oxalate de chanx.

La masse libéro-ligneuse a la forme d'une ellipse, sur une coupe trann sale. Elle est composée de liber et de bois; on y remarque nonseulement du liber externe, mais encore du liber qui sappuie contre la partic trachéenne du bois. Le liber externe placé à la face inférieure se compose d'une ou deux conches de fibres libériennes (c) it membrane épaissie, mais avec lumière centrale très grande. Ces fibres sont polygonales sous une coupe transversale. Au-dessus des fibres se trouve le liber' mou beaucoup plus développé (d).

Le bois (e) est composé inférieuremont d'un massif puissant de séries radiales de fibres ponctuées à membranes très épaisses. Dans ce tissu fibreux se remarquent quelques rayons médullaires. Supérieurement, se trouvent de faibles ilots de valisseaux spiralés et trachéens. Ces ilots s'avancent dans du tissu cellulare formant quelques couches et représentant la moelle. 
Contre la partie supérieure du tissu mólullate s'uppuie un amas de

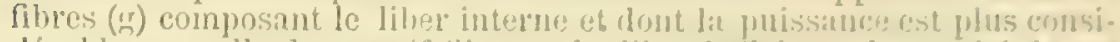

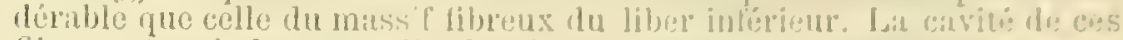
fibres est moindre rue cel! des fibres externes, et les mambrancs en

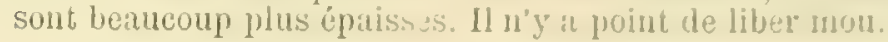

IAMILLF DLS AIISTOLOCHIVIS.

Feuille d'Asaret d'Europe. - Asarum curigaum. L.

1. Lame intemeurale. a. Epirlerme. - A la litce supérieure et at la face inférieure se trouve une couche d'épiderme dont les ítentnts pré. sentent un contonr simeux et des parois munies de monctuations. I sit surface épidermique est revetue d'une membrane cuticularisie assez épaisse.

Au milieu des cellules, très rrandes et incolores, sont des éléments plus petits. Ces derniers, vus de face, sont arrondis ou polyonaux : si on les examine sur une coupe transversale, ils ont lat forme d'un rectangle dont les grands côtés sont perpendiculaires it la surface de la feuille.

Ces cellules renferment une oléo-r’sine qui jaunit sous l'action de la potasse.

Les deux épidermes sont pourvus de stomates et de poils. Les poils coniques sont composés d'une série de cellules dont la hauteur augmente de la base vers le sommet. La cellule supévicure est conique; l'extrémité en est tres souvent obtuse. Lat membrane des poils, un peu épaissie. est parsemée entièrement de tubercules arrondis.

b. Mésophylle. - Le mésophylle vert est hétérogène. Il est dépourvi de cellules cristalligènes.

Contre l'épiderme supérieur existe une assise de grandes cellules vertes, cylindriques et perpendiculaires it leppiderme. Ces cellules sont généralement trois fois plus longues que larges. Au-dessous de ce parenchyme en palissade se trouvent $3-5$ conches parallèles de cellules qui, sur une coupe transversale, sont allongées parallélement à la sur face foliaire et laissent entre elles de nombreux méats.

II. Nervure. - L'épiderme, dépourvu de stomates, mais muni de poils semblables i ceux qui existent sur la lame, est constitué it lia face inférieure et à la face supérieure par une couche de cellules allongées suivant l'axe de la nervule. La membrane de ces cellules, épaissie et ponctuée, est recouverte superficillement d'une cuticule qui présente des crêtes assez saillantes.

Au-dessous de l'épiderme supérieur se trouvent deux ou trois assises de cellules un peu épaissies et contenant des grains de chlorophylle: - elles sont prismatiques ì base rectangulaire. La partie inférieure de ce tissu est en contact avec un faisceau libéro-ligneux où le liber a ses éléments cellulosiques, où le bois est composé de raisseaux ponctués, spiraux et de trachées. Contre la face inférieure du liber s'appuie du parenchyme dont les cellules incolores ou renferm tut très peu de chlorophylle, laissent entre elles des méats. Elles on: la forme de prismes allongés à base polygonale. Ce tissu repose sur deux ou trois couches de cellules très épaissies aux angles. 
FAMILLE DES LAURINÉES.

Feuille de Lauricr d'Apollon. - Laumus nobilis. L.

I. Lame intemeurale a. Epideme. - L'épiderme glabre est constitué par une seule assise de cellules à membrane très épaissie, ponctuée. Elles sont tabulaires, présentent superficiellement une paroi sinueuse et sont levêtutes d'une couche épaisse de cuticule qui s'avance mème entre les parois latérales. L'épiderme inférieur seul porte des stomates.

b. Mésophylle. - Le mésophylle est coloré par la chlorophylle. A la face supérieure existent deux couches de cellules cylindriques disposées en palissade et à membrane un peu épaissie. Dans l'assise superficielle, les éléments sont plus allongés que dans l'autre; ils ont une longueur' de trois à quatre fois plus grande que leur largeur. A la face inférieure les cellules sont rameuses, elles laissent entre elles des méats et contiennent de gros grains d'amidon; observées dans une section longitudinale, elles semblent arrondies, carrées ou rectangulaires. Dans le mésophylle se trouvent de grosses glandes arrondies ou polyédriques, unicellulaires, renfermant une huile essentielle jaunà tre ; elles sont pour la plupart situées dans le parenchyme muriforme.

II. I Iarge. - Lépiderme marginal reconvre un cordon de gros éléments à membrane épaissie, à cavité grande, qui contiennent de gros grains d'amidon.

III. Nervure. - En dedans de l'épiderme à éléments presque cubiques, siège sur les deux faces un massif de grandes cellules collenchymateuses très épaissies et ponctuées sur leur paroi, au milieu desquelles existent parfois des glantes unicellulaires. En dedans du collenchyme se trouvent de grandes cellules à parois lignifiées, épaissies et ponctuées ayant la forme d'un prisme droit à base polygonale (5 à 6 cótés).

C'est contre les assises de ce tissu lignnifié que s'appuie un are libéro. ligneux très étendu latéralement et composé on allant de In partie inférieure de la nervure ver's la supérieure: $1^{\circ}$ de liber constitué inférienrement par des fibres lignifiées arrondies (en section transversale) et supérieurement par du liber celluleux au milieu duquel sont placées des cellules oléo-résineuses; $2^{0}$ de bois en général un peu moins développé que le liber et formé de bandes rayonnantes vasculaires séparées les unes des autres par une ou deux rangées de cellules épaissies. lignifiées et aplaties contenant de gros grains d'amidon; 30 de fibres ana ogues aux fibres libériennes inférieures, mais un pen moins épaissies.

Les éléments qui constituent la nervure présentent la particularitẻ de contenir des grains d'amidon. On en trouve dans l'hypoderme, le tissu lignifié fondamental, les fibres libériennes et les rayons médullaires

FAMILLE DES SILÉNÉES.

Feuille de Saponaire officinale. - Saponaria officinalis. L.

I. Lame interneurale. a. Epiderme. - L'épiderme supérieur est constitué par une seule couche de cellules tabulatres, sinueuse à la surfice et rectangulaires en section transversale. Elles sont recouvertes 
d'une couche de cuticule presentant des critos Inngitudinales tris finos

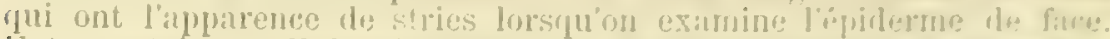

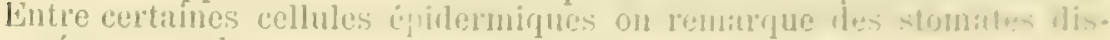
posés sáns ordre.

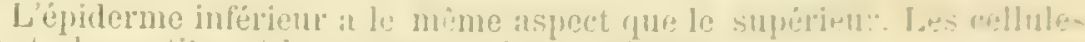
sont plus petites et les stomates pus mombrenx.

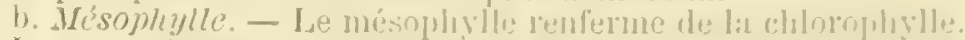

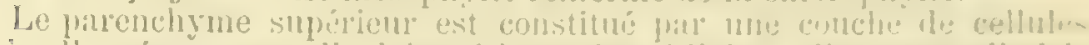

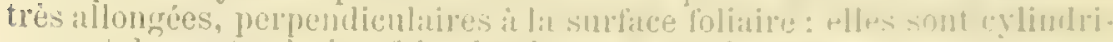
ques et de quatre il cinq lois plus longues que langes.

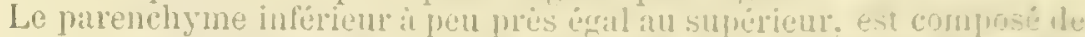
trois it quatre assises de cellules rameuses yni, un culpue tratustersale, sont rectimgulaires, et présentent de laco trois: juatre: cing branches tris courtes et disposées irruliemement.

Entre les deux parenchymes se trouvent de crosses rellules isolex sphériques ou polyédrigues incolores. contenint chacme une masse do cristanx doxalate de chanx.

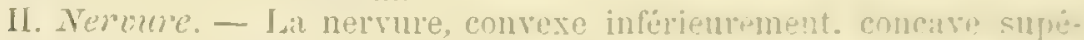
rieurement, est limitie en hant et en bas pit une conche dre crlules épidermiques tabulailes, hexaronales et déponvues do stomites. In. dessus de l’épidermo infériem existent me on drox couches dr cellutes collenchymateuses, de denx it quatre fois julus Inngues yue latres. prismatiques a base polyonale et a parois munirs de fables fonctuations étendues transrersalenent. Au-dessous de lépiderme suptrieur se trouve une couche de cellules ponctuces, tries longues. ipaissies. L'espace situé entre les deux hyodermes est rempli par du parenchyne au milieu duquel on voit un are libéro-ligneux dont lat concavitis supérieure est occupée par une espece de moelle. Cette masse pranchymateuse de remplissare est en majorité constituce par des cellules incolores, le parenchrme vert de la lame ne dépassant guere lat lingle te jonction de la lame et de la nervure. Les cellules incolores, plus considérables a la face inférieure qu'a la face supérienre. misqu'il nen existe i cette dernière que de deux à sept, tandis que lit premiere en possule de huit a dix, ont la forme d'um prisme droit dont lit base est polyonale régulière (de cing ì huit côtés) : elles sont percées de lérréres poncluations transversales et la hauteur en est de deux it six fois superiemre a la largeur.

L’are libéro-ligneux est composé inférieurement de liber: supérieurément de bois.

Le liber présente à sa fice inférieure deux ou trois conches continues de fibres cellulosiques it section polygonale, dont les membranes sont épaissies au point de contact. Ce parenchyme se continue en laut par le liber mou constitué par plusieurs fibres ralliales de six á dix petits éléments dont les membranes son épaissies.

Le bois, qui n'est guère plus puissant que le liber, présente des rais. seaux spiraux et trachéens. Ces éléments sont disposés en un grand nombre de files radiales et forment plusieurs grounes separés par de petits rayons médullaires.

Dans la concavité supérieure du bois se rencontrent plusieurs couches de cellules (moelle) ne laissant entre elles aucun méat, très allongres. prismatiques ì base polygonale. Leur's membranes ponctuées sont éraissies surtout au point oil elles se mettent en contact. comme le fitit un collenchyme. C'est une moelle collenchymateuse. 
FAMTLLE DES CRUCIFERES.

Feuille de Cresson de fontaine. - Nesturtium officinate. $\mathrm{R} . \mathrm{Br}$.

I. Lame intemenrale a. Epideme, - Lépiderme glabre est composé d'une couche de cellules dont le contenu est très sinueux, et dont les membranes sont minces. L'épiderme inférieur est seul pourvu de stomates.

b. Mésophylle. - Le mésophylle hétérogène ne renferme point de cellules cristalligènes. A la face supérieure siègent ordinairement deux assises de cellules cylindriques perpendiculaires à la surface de lir feuille. Les cellules les plus superficielles sont deux fois plus longues que larges. Au-dessous du parenchyme en palissade se trouvent quelques couches parallèles de cellules qui, sur une coupe transversale, paraissent rectangulaires ou elliptiques. Elles sont allongées parallèlement ì l'épiderme.

II. Nervure. - Les cellules de l'épiderme sont allongées suivant l'axe de la nervure. Entre les deux épidermes il y at un parenchyme dans lequel plonge un faisceau libéro-ligneux. Le parenchyme est composé de cellules dont les membranes sont ninces, et qui renferment des grains de chlorophylle. Ces derniers sont surtout nombreur dans les cellules voisines de l'épiderme supérieur. La section transversale de ces éléments est assez crande et de forme polygonale.

Le cordon libéro-ligneux est elliptique ì grand diamètre latéral (coupe transversale). Son liber en are est formé d'éléments dont les parois sont cellulosiques. Le bois, souvent convere à sa partie supérieure, est composé de trachées et de vaisseaux spirüux.

Feuille de Cochléaire oflicinale. - Cochlearia officinalis. L.

La nervure du Cochlearia ressemble à celle du Nasturtium. Il n'y a de différence que dans l'épiderme et le mésophylle. L'épiderme infé. rieur a des cellules fortement smeuses, tandis que les cellules de l’épiderme supérieur présentent des sinus peu profonds.

Les cellules qui touchent l'épiderme inférieur sont rameuses lor'squ'on examine la coupe transrersale de la feuille (pl. V. fig. III, c). Ce caractère n'existe pas dans le Cressnn.

PAMILLE DES RENONCULACÉES.

Feuille d'Aconit napel, - Aconitum napellus. L.

I. Lame interneurale. a. Epiderme. - L'épiderme supérieur, dépourvu de stomates et de poils, est composé de grandes cellules un peu sinueuses quand on les voit de face; rectangulaires ou carrées si on les observe dans une coupe transversule. Leur membrane un peu épaissie est recouverte superficiellement d'une mince couche de cuticule.

L'épiderme inférieur prẻsente des cellules semblables à celles de l'épiderme supérieur mais moins larges. De plus, il y a des stomates 
arrondis ou elliptiques disposés irrénulicrement entre quatre ou six cellules d'épiderme.

b. Mésophylle - Le mésophylle vert hétérogiene est composé surué. rieurement de deux couches de tissu en palissade it grandas collules cylindriques dont les plus grandes ont une largeur six fois moindre que la longreur et quatre fois moindre que lat longruenr des crallules épidermiques supérieures. Infériemement, le mésophylle est constitué par quatre ou cind couches imérnlieres de grandes cellules rameuse. formant une lame un peu plus développée que la lame de tissu muriforme. La coupe transversale de la feuille montre ces cellules allongées parallèlement it la surface de l'organe.

II. Marge. - La marge est contituée par une couche de cellules épidermiques contre laquelle vient se terminer le mésuphylle vert.

III. Nervure. - La nervure, convere inféricurement, concave supërieurement, est munie it ses deux faces de deux épidermes (pl. III, fig. II, a) dépourvus de stomates et clont les cellules sont talulaires. Ces dernieres, recouvertes d'une couche moyenne de cuticule. sont tres allongées. De face, elles ont d'ordinaire la forme d'un hexagrone it deux côtés supérieurs petits, et quatre latéraux plus rrands. Les deux couches épidermiques enveloppent le tissu cellulaire neural (c, d) dans lequel se trourent placés d'ordinarre trois cordons médullo-libérolioneux assez distants l'un de l'autre, et dont le médian est le plus considérable. Chaque cordon $(d, c)$, vu transversalement, a la forme d'une ellipse ì grand diamètre inféro-supérieur. Il cst composé en allant de bas en hant :

$1^{\circ}$ D'un liber (d) constitué inférieurement par des fibres allongres, it contour arrondi et à parois cellulosiques minces, supérieurement par des cellules libériennes petites, mais très épaissies, arrondies, sourent allongées tangentiellement. Elles torment un amas qui se présente sur une section transversale sous l'aspect d'une lentille bi-convexe.

20 D'un corps ligneux (e) qui, concave en bas. convexe en haut. est constitué par des vaisseaux rayés, spiraux, trachéens. Ce bois est beaucoup plus déreloppé que le liber celluleux.

30 D'une sorte de moelle (f) dont les cellules cylindriques it paroi munie de ponctuations, sont très épaissies à leurs points de contact. comme dans le tissu collenchymateux.

La masse parenchymateuse située entre les deux epidermes est composée de collenchyme, d'un parenchyme incolore et d'un parenchyme vert. Le collenchyme $(b, h)$ qui existe à la face inférieure et supérieure. tout contre l'épiderme, forme une ou deux couches de cellules faiblement épaissies à leurs points de contact; ces cellules sont très allongées, prismatiques, et munies sur leur paroi de ponctuations étendues latéralement. Le parenchyme incolore (c) constitue le massif celluleux le plus considérable de la nerrure; ses cellules très allongées sont cylindriques ou prismatiques, it base polygonale. Elles sont pourrues de ponctuations sur leur membrane; la largeur de leur base est d'autant plus grand qu'elles sont plus roisines de l'épiderme inférieur. Quant au parenchyme rert, il est peu déreloppé dans la nervure, il n'atteint jamais le plan médian de celle-ci.

Feuille d'Aconit tue-loup. - Aconitum lycoctonum. L.

L'Aconit tue-loup se distingue principalement du Norpel par la pré- 
sence sur l'épiderme supérieur et l'épiderme inférieur de poils unicellulaires coniques. Ces dernier's, un peu recourbés au sommet, étranglés au niveau de l'épiderme, ont leur base enchàssée entre des cellules épidermiques.

Feuille d'Aconit anthora. - Aconitum anthora. I.

Les cellules épidermiques de l'Aconitum anthora ont, comme celles des deux précédents, leur's membranes minces ; mais l'épiderme porte des poils unicellulaires dont les parois sont munies extérieurcment de nombreux tubercules arrondis.

Feuille d'Aconit bicolore. - Aconitum variegatum. L.

Les cellules épidermiques ont les parois un peu épaissies et nettement ponctuées. Les poils unicellulaires sont lisses.

\section{FAMILLE DES BERBERIDÉES.}

Feuille de Berberis commun. - Berberis vulgaris. L.

I. Lame interneurale. a. Epiderme. - L'épiderme inférieur est composé d'une assise de cellules tabulaires aplaties de haut en bas, à surfice sinueuse, et recourertes superficiellement d'une couche de cuticule. Entre les cellules se trouvent des stomates elliptiques, disnosés irrèculièrement entre trois, quatre ou cinq cellules épidermiques. Les cellules de l'épiderme supérieur n'enferment aucun stomate; elles ressemblent à celles de l'épiderme inférieur', toutefois elles sont plus petites.

b. Mésophylle. - Le mésophylle vert situé entre les deux épidermes est hétérogène. A la face supérieure il constitue deux couches de cellules en palissade, cylindriques, pressées les unes contre les autres; ces cellules ont une longueur qui ne dépasse guère le triple de leur largeur. Le mésophylle inférieur, deux fois plus développé que le supérieur, possède cinq ou six assises de cellules rameuses qui, en coupe transversale, paraissent rectangulaires, allongées suivant la surface foliaire. De face, elles présentent trois ou cinq prolongements. Les méats qu'elles laissent entre elles sont très irréguliers, polyédriques.

II. Marge. - Contre l'épiderme s'appuie un cordon demi-cylindrique constitué par des fibres a section polygonale (de cinq à sept côtés), à parois épaisses pourvues de petites ponctuations arrondies et à lumière considérable.

III. Nervure. - L'epiderme supérieur et l'épiderme inférieur, dépourvus de stomates, présentent des cellules tabulaires allongées dont la surfice est polygonale. Le centre de la nervure est occupé par trois ou cinq faisceaux libéro-ligneux qui forment ensemble un arc dont la concavité est tournée vers la face supérieure de la feuille. Chaque cordon est composé de liber et de bois; le liber (pl. VII, fig. IV, a) inférieur celluleux a l'aspect d'une lentille bi-convexe lorsqu'on le regarde transversalement. Le bois (e) a la forme d'un triangle à sommet supérieur' et à base inférieure concave, conposé de vaisseaux trachéens, spiraux et ponctués. Tous les faisceaux sont plongés dans une masse très remarquable de tissu lignifié b). Cette derniere se présente sous la forme d'un as de 
pique dont les lobes sont fournes vers la tace inlievieure do la nerware.

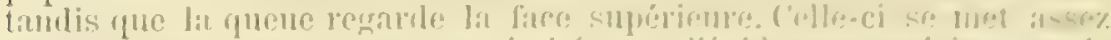

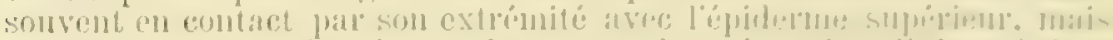

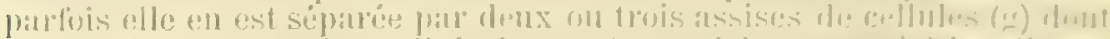

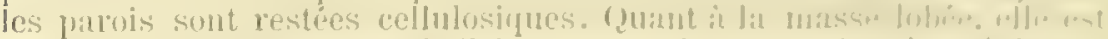

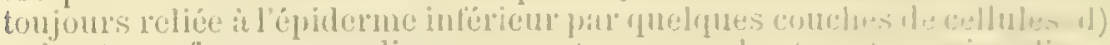
gut ont une forme arondie en coupe transversate et sont Intures d'un. membrane composée de cellulose.

Le massif lignifie est constitue par des edements dont les jarois un peu épaissies forment de petites ponctuations. Sur nut cunpu transversale, ces éléments sont polygoniux ì cincl ou six angles. Sur une coupe longitudinale, ils sont allongés, placés les uns atu-dessus des autres et séparés par des cloisons obliques.

\section{PAMILLE DES CORLARIEES}

Fcuille de Coriaire a feuilles de myrte, (Redoul). - Coriarin myrtifulir. L.

1. Lame interneurale. - L'épiderme glabre et muni de stomates anx denx faces est composé d'une assise de cellules tabulaires it controur polyronal et à membrane assez mince. Les stomates. trés allungés sont macés latéralement entro deux cellules épidermiques. Au-rlessus de l'épiderme supéricul' existent deux couches de cellules cylinhrifurs disposées en palissade; les plus superficicles sont deur ou trois fois plus longues que larges.

A la face inférieure, le mésophylle forme quatre ou cinu conches de cellules rameuses, qui, en coupe transversale, paraissent arrondies. rectungulaires ou elliptiques, la plupart allongées parallelement it la surface foliaire, tandis que de face, elle présentent des branches extrémement courtes et laissent entres elles de faibles méats. Sons lanction des sels de fer, ces cellules bleuissent; elle renferme done du tamnin.

II. Maige. - La marge est constituée uniquement par la terminaison latérale du mésophylle et de l'épiderme.

III. Nervure. - La nervure est biconvere. L'épiderme (pl. VI, tìr. II. $a, g)$, dépourvil de stomates, est constitué par une couche de cellules qui, examinées de face, sont polygonales. Les parois de ces cellules. épaissies et légèrement ponctuées, sont revètues superficiellement d'une cuticule portant des crêtes qui ont l'aspect de stries, lorsqu'on observe la surface de l'épiderme. En dedans de l'épiderme siège un massif de cellules pâles à membrane épaissie, ponctuée, cellulosique $(b, f)$. Elles ont un contour polygonil et, sur une coupe longitudinale, elles ont la forme d'un polygone très-allongé parallèlement à l'are de la nervure. C"est dans cet amas de parenchyme que se rencontre un cordon dont la section est elliptique à grand diamètre latéral ; ce cordon ne touche point directement les cellules du parenchyme indiqué, il en est séparé par une conche de cellules formant tout autour de lui une espèce de gaine (c) : les éléments de cette assise protectrice ont une surface transversale. presque rectangulaire. Le cordon est composé inférieurement d'un aro continu de fibres libériennes à parois peu épaisses et cellulosiques (b). Au-dessus de ces fibres se trouve le liber celluleux dont les éléments renferment du tannin. Le bois (e) est constitué par quelques bandes de trois à six vaisseaux, rayonnantes, irrégulières et séparées par des 
rayons médullaires formés de un ou deux rangs de cellules å membrane mince etcellulosique. A la partie supérieure du bois siẻge un amas de cellules au contour polygonal; ces cellules sont disposées en files verticales et séparées les unes des autres par' des cloisons horizontales.

\section{FAMILLE DES RUTACÉES.}

Espices : Ruta grateolens, L. - Ruta montana. - Barosma. - Empleurum.- Pilocarpus pennatifolins. Lem. - Ptelea trifoliata. L.

I. Lame interneurale. a. Epicerme. - Les cellules épidermiques sont tabulaires; la surface en est polygonale dans la Ruta montana L., le Barosma, l'Empleurum, le Pilocarpus, le Ptelea; légèrement sinueuse dans la Rue fétide. Les cellules du Ptelea et du Barosma renferment des sphéro-cristaux d'inuline. La paroi profonde des cellules épidermiques du Barosma et de l'Emplermu se gonfle considérablement sous l'influence de l'eau; elle est mucilagineuse. L'épiderme est glabre, sauf dans certaines feuilles de Pilocarpus dont la face infés rieure présente des poils coniques unicellulaires.

b. Mésophylle. - Le mésophylle, toujours hétérogène, renferme des glandes multicellullaires et des cellules cristalligènes. Ces dernières ne manquent que dans l'Emplequm. Les glandes sphériques (Ruta, Barosma, Ptelea) ou ellipsoïdales (Pilocaipus) sont entourées de deux ¿̀ quatre couches de cellules disposées concentriquement; elles renferment de l'huile essentielle ordinairement jaunâtre.

Le mésophylle rert forme à la face supérieure une couche de tissu en palissade daus le Pilocarpus, le Barosma, le Plelea. Il existe au contraire deux ou plusieurs assises dans les Ruta. Le parenchyme infél'iem est méatique.

II. Nervure - L'épiderme, ordinairement dépourvu de stomates, est glibre daus les Ruta, l'Empleumom et certaines feuilles de Juborandi. D'autres feuilles de Pilocaimus ainsi que le Ptelea possèdent des poils unicellulaires sur l'épiderme inférieur. La face supérieure des Barosma est munie seule de ces appendices.

Les deux épidermes renferment du parenchyme qui donne naissance ì des glandes et à des éléments cristalligènes et dans lequel sont placés un ou plusieurs cordons libéro-ligneux. Ce parenchyme homogène it cellules épaissies et incolores dans le Barosma, varie dans la Ruta grareolens; à la face supérieure se trouvent deux ou trois couches de cellules vertes disposées en palissade, tandis qu'à l'inférieure il y a un petit amas de cellules incolores.

Lo système fibro-vasculaire, représenté par un seul arc inférieur dans le İcrosma, l'Empleurum et la Rula, est au contraire composé de deux bandes libéro-ligneuses, l'une supérieure, l'autre inférieure, dans le Pilocarpus et le Plelea. Les fibres libériennes du Barosma, de l'Emplevirum et du Pilocarpus sont lignifiées; celles du Ptelea et des Rula ont la membrane cellulosique.

Les feuilles des espèces de cette famille possèdent les caractères communs suivants:

Epiderme glabre, ou muni de poils unicellulaires. Point de poils plinduleux, Des glandes internes, des cellules cristalligenes au moins dans le parenchyme neural. 
Rue fítide. - Ruta gratoolens. I.

I. Lame interneurale. at. Epidème. - Lüpiderme infériour glabre

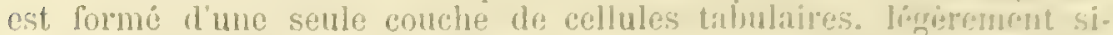
nueuses. Cet épiderme, recouvert d'une couche de cuticule, porte des stomates disposés irrérulierement. I aid pluprert sont situes entre quatre

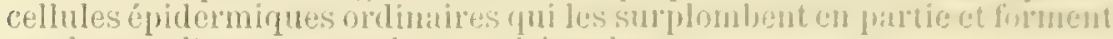
au-dessus d'enx un canthl ou antichanulur.

L’épiderme supérieur, déponrvu de stomates, est constitué par des collules it parois moins sinuenses.

b. Mésoplylle. - le mésophylle rert est hétirorine. Il est remálquable en ce qu'il renferme des flindes multicellulatires arrondies: ces dernières viennent toncher l'épiderme supúriell. A la face supérieure. le mésophylle forme deux couches de cellules cylindriques disposies en palissade et de trois a quatre fois plus longues que larges. Sur la face inférieure, les cellules sont ramenses et laissent entre elles des nuéats. La couche cellulaire qui tonche immédiatement l'épiderme inféricm' est peu rameuse. Ces cellules, allongées perpendiculairement it la surface foliaire si on les observe sur une coupe transversale, sont de deuxet demie ì trois fors plus longues que larres. lérerement coniques ef produisent lateralement des prolongements tres courts; vues de face. elles semblent arrondies. I,es cellules situées entre cette conche et los béments en palissade, présentent deux ou trois branches et sont dis. posées irrigulierement. Filles laissent entre elles de grands méats.

C'est surtout dans ce dernier tissu que l'on rencontre dens sortes dorganites: 10 des cellules cristalligines; 2a des glandes multi-cellulaires. Les cellules cristalligènes sont sphériques; elles contiennent des arglomérations de cristaux d'oxalate de chaux. Elles se trouvent anssi bien dans lintéricur du tissu rameux qu'au voisinage du tissu en palissade.

II. Marge. - La marge est formée de parenchyme vert et dépiderme. On pent y rencontrer une glande séparće de l'épider'me par une conche de collules vertes.

III. Nervure. - La nervure, à peine apparente il lextériem, est limitée en haut et en bas par une couche de cellules épidermiques allongées suivant la longueur de la nervure et dépourvues de stomates.

Au centre de la nervure sière un cordon ì section transrersale elliptique. Ce finisccau est composé de trois parties. L'inférieure, liberricnne, forme un rroupe d'éléments ì parois assez minces et cellulosiques; la moyenne, ligneuse, moins puissante que la précédente, est composce de quelques files vasculaires (raisseaux trachéens et raisseaux spiraux séparées les unes des autres par un ou deux rancrs de cellules dont lit section transversale est polygonale. Quant à la portion supérieure, elle constitue un imas de collules allongées, prismatiques, it base carrée ou polygonale.

Le coldon que nous venons de décrire est compris entre des éléments cellulaires de deux sortes. Il est en contact, supérieurement, arec deux couches de parenchyme vert en palissade qui se continuent latéralement avec le tissu supérieur de la lame; sa portion libérienne touche à un massif comprosé de trois à cinq assises de cellules; celles-ci présentent des parois cellulosiques un peu épaissies. Sur une coupe transter'sale, clles sont arrondies ou polygonales à angles émoussés, et laissent entre elles de petits méats dont la plupart sont rectangulaires. 
Rue de montagne. - Rutc montand. I.

Les cellules épidermiques ont un contour polygonal, et il existe il la face supérieure et it la face inférieure deux ou trois couches de cellules en palissade.

Feuilles de Buchu ou Bucco. - 1. Bucco large (1). - Barosma betulina. Bart.

I. Lame intemeurale. a. Evidéme. - L'épiderme est constitué par une assise de cellules tabulaires recouvertes d'une épaisse couche de cuticule. Un lambeau d'épiderme, placé sous le microscope, nous montre les cellules polygonales et dépourvues de sinuosités.

Les cellules de l'épiderme supérieur présentent une face profonde douée de propriétés spéciales.

Cette portion de la membrane se gonfle considérablement sous l'influence de l'eau : elle se transforme en mucilage. La cavité de ces éléments renferme en outre de petits sphéro-cristaux d'inuline.

Les stomates, qui ne se rencontiont que sur la face inférieure de la feuille, sont disposés sans ordre; ils sont arrondis et entourés de quatre ì six cellules épidermiques ordinaires

b. Mésophylle. - Le mésophylle vert est hétérogène. Le supérieur: moins développé que l'inférieur, constitue une seule couche de cellules cylindriques très serrées les unes contre les autres, et de cinq à six fois plus longues que larges.

Le parenchyme inférieur est formé de plusieur's couches de grandes cellules très rameuses (de trois à 5 branches; laissant entre elles de grands méats. Ce parenchyme est parsemé de cellules arrondies ou polyédriques renfermant des agglomérations de cristaux d'oxalate de chaux. On y rencontre de plus de grosses glandes arrondies, multicellulaires, roisines de l'épiderme inféricur.

II. Marge. - La marge, constituée par des cellules vertes, renferme de très grosses glandes. L'épiderme qui recouvre les dents est formé de cellules dont les membranes épaissies présentent de nombreuses ponetuations.

III. Nervure. - La nervure, un peu convexe, est limitée par une couche de cellules épidermiques ì membrane épaisse, rectangulaires superficiellement. L'épiderme supérieur est muni de poils courts, unicellulaires, de trois à cinq fois plus long que larges, coniques et ì surface tuberculeuse. Au dessus de l'épiderme inférieur existent quatre ou cinq couches de cellules à membrane épaisse, ponctuée et ì caritẻ considérable. Ces cellules rectangulaires en section longitudinale, sont arrondies ou carrées sur une section transtersale. La couche supérieure touche à un cordon central en forme d'arc constitué par du liber inféricur, du bois et de la moelle.

Le liber est formé inférieurement d'un puissant massif de quatre à six couches de fibres lignifiées, arrondies (section transversale), à

(1) M. de Lanessan. Bull. Soc. Limn. Paris, 1877, et traduction de A. Fluckiger. Loc. cit. tome I., page 200. Les figures du Buchu, domées dans ce dernier ouvrage, montrent deux couches de cellules à l'épiderme supérieur. Nous n'avons constaté qu'une assise. La paroi mucilagineuso profonde a été prise pour une assise de cellules. 
membrane epaisse, ne laissant qu'une laible cavitio interne; supiriourement, de liber non moins développé yne le liber fibreus.

Le bois, presque aussi puissant que le liber, ast compros dre phucienrs handes fibro-vatsculares séparées les unes des antres palo de minces rayons médulaires composés d'une seule rangre de cellules tris aplaties tangentiellement. Charue bande est formen alo fibms tres épaisses it section transversale carlée et de valissuatux mpiranx. trachées).

La moelle, supérieure au bois, jossede des cellules cylindrique: allongées et dont les membranes lignifiées, épaissies, sont pourues de nombreuses ponctuations.

Au-dessus de l'are libéro-ligneux existe sur la lirne médiane un petit ilot it section rectangulaire ou carrée, ilot formé de deux rangrés de cellules cylindriques presque incolores. Sur les parois lacirales de cet ilot vient s'appuyer du parenchyme vert qui se continue de chaque côté avec le tissu en palissade de la lame.

\section{Buchu long. - Barosma serratifulia. Willd.}

Le Buchu long ofrie ì 'peu près les mêmes caracteres que le précédent. Nous n'y avons trouré de différence que dans l'épiderme rul revêt les dents marginales. Les cellules épidermique du Buchu long ont une membrane plus mince présentant des ponctuations tres pell visibles.

\section{Limpleurum servilatum. Aiton.}

Les feuilles de l'Empleurum serrulatum se distinguent des feuilles du Barosma par l'absence de poils sur l'épiderme neural et de cellules cristalligènes dans le mésophylle.

Pilocarpe à feuilles pennées. - Jaborandi. - Pilocaryus pennatifulius. Lemaire.

I. Lame interncurale. a. Epiderme. - L'épiderme supérieur. dépourvu de stomates, forme une seule couche de cellules épaisses, tabulaires, ì surface irrégulièrement polygonale. Il est reconvert d'une couche épaisse de cuticule présentant de fines crêtes saillantes qui ont l'aspect de stries lor'squ'on examine de face un lambeau épidermique.

L'épiderme inférieur, plus aplati que le supérieur, donne naissance is des stomates placés irrégulierement. Ceux-ci, presque aussi larges que longs, sont bordés latéralement par une cellule en forme tle croissant.

Certaines feuilles ont les deux fices glabres. D'autres sont poilues inférieurement. Les poils sont alors coniques, unicellulaires, et ont une paroi épaissie au point que la cavité est très minime. Leur base est en. chàssée dans l'épiderme.

b. Mésophylle. - Le mésophylle, composé en grande partie de cellules contenant de la chlorophylle, renferme des glandes pluricellulaires (pl. V, fig. IX, d) et des cellules a cristaux (c). Le parenchyme en palissade (b) constitue une assise de petites cellules cylindriques pressées les unes contre les autres, et de trois it quatre fois plus longues que larges. Quelquesune de ces cellules vertes contiennent des cristaux agglomérés d'oxalate de chaux.

Au-dessous de ce parenchyme se trouve un tissu (e) au moins trois fuis plus développé que le précédent. Les cellules en sont arrondies, 
rectangulaires ou ramenses; elles laissent entre elles des espaces assez volumineux. Ce parenchyme contient des éléments à cristaux, surtout très nombreux au roisinage du tissu muriforme et de l'épiderme inférieur (c).

De grosses glandes pluricellulaires ellípsoïdes à grand diamètre parallèle il la surface épidermique, existent aussi bien dans le parenchyme supérieur que dans l'inférieur. Elles sont toujours très rapprochées de l'épiderme.

II. Marge. - On rencontre au bord de la feuille du parenchyme vert recouvert d'épiderme.

III. Nervure. - La nerrure est limitée de haut en bas par de l'épiderme à cellules épaissies, rectangulaires superficiellement. Il est tantôt glabre, tantòt pourvu de poils qui présentent les mêmes caractères que ceux de la lame.

Contre l'épiderme inférieur siège une masse cellulaire puissante. Les cellules grandes sont allongées, cylindriques ou prismatiques, à base régulièrement polygonale. Elles laissent entre elles de petits méats et forment de huit à dix assises, dont la supérieure est en contact avec un cordon. On remarque dans ce tissu des glandes pluricellulaires.

En section transversale, le faisceau a la forme d'un demi-cercle. Il est en majeure partie composé d'un arc inférieur à concavité tournée en haut et d'une bande supérieure parallèle à la surface foliaire. Cet arc et cette bande, constitués par du liber" et du bois, laissent entre eux un espace occupé par du parenchyme.

Des tibres et des ceilules entrent dans la composition du liber.

Les fibres longues, à section polygonale, ont des parois épaissies et lignifiées, d'où une cavité centrale peu considérable. Elles ne forment point un arc continu, mais se groupent en ilots de puissance inégale. Quant au liber mou, il est composé de cellules séveuses et n'est pas aussi épais que le liber parenchymateux. Le bois est constitué par plusieurs bandes rayonnantes séparées entre elles par une rangée de petites cellules. Il existe dans chaque bande, en allant du liber vers le parenchyme interfasciculaire, des fibres ponctuées très-épaissies, des vaisseaux ponctués, rayés, spiraux et des trachées.

Dans la moelle, les éléments cylindriques, sont allongés parallèlement à la nervure. Leur membrane est épaissie, ponctuée et souvent lignifiée.

Au-dessus du cordon se trouvent quelques couches de parenchyme semblable à celui qui existe au-dessous du faisceau. Entre ce tissu et l'épiderme supérieur règnent deux ou trois assises de petites cellules dont la section transversale est carrée ou rectangulaire. La plupart renferment de la chlorophylle; d'autres contiennent des agglomérations cristallines. On rencontre aussi dans ce tissu de volumineuses glandes pluricellulaires.

\section{Autres espèces de Jaborandi.}

Les habitants de l'Amérique du Sud donnent le nom de Jaborandi à des plantes diffërentes des Pilocarpies: ce sont le Scrronia Jaborandi Gaul et le Poirre réticulé, Piper veticulatum, de la famille des Piperacées (1).

(1) Voir H. Baillon au Journal de Parmacie et de Ghimie, 185, XXI; Bulletin de la Société linéenne de Paris, $18 \%$. 


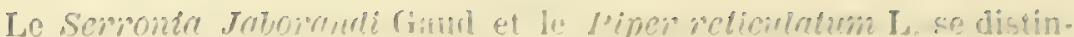
gnent du Pilocriphes par un grand nombro de caractives dont les frincipaux sont les snivants: absence de ghanden pluricollulares a des frandes unicellulatires: tissu vent situó anx denx faces futro donx ou frois couclics de collules intorloress.

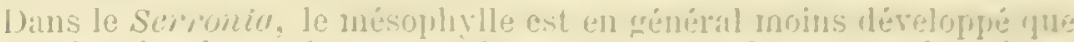
les assises incolores. Son parenchyne en patiosude est represente prar une couche de cellules dont la longhenr ne depasse jamais la larmetur. Au-dessus de l'hypoderme inférieur sierrent des fibres it parois lienditiees. Les faisccaux libéro-ligneux sont disposés en ato rlont lat concarité est en lant.

Le parenchyme en palissade du Piju' róliculaluin L. forme une cou. che de cellules aux moins deux fois plus longres que latres. I.e paren. chyme rameux est égal ou inférieur en juissance an tissu muriforme. Il n'y a pas de fibres limnifiées au-dessus du collenchyme inférieur.

Ptélée a trois folioles. - Plelen trifotiatr. L.

I. Lame interneurale. a. Epiderme. - L'épiderme supérieuret l'épi. derme inférieur sont à une seule couche de cellules tabulaires it membrane peu épaisse, et à surface irrécrulièrement polyconale. Ëlles contiennent des sphéro-cristaux d'inuline. Lépirlerme infürieur est seul pourvu de stomates; de plus, il porte aux petites nervures des poils coniques recourbés, unicellulaires, beancoup plus longs que larges (pl. I, fir. V).

b. Mésophylle. - Le mésophylle est hétérorene. La partie supérieure est constituée par une couche de cellules cylindriques pressées les unes contre les autres, de quatre ir six fois plus longues que larres. Le parenchyme inférieur, environ deux fois plus dérelopné que le supérieur. a les cellules légrerement rameuses, et sur une coupe transversale ces cellules paraissent arondies ou rectanualares it longs cutés parallëles it la surface. On rencontre dans ce parenchyme des cellules arrondies contenant des agglomérations de cristaux d'oxalate de chaux. Dans les deux couches du mésophrlle on remariue des glindes pluricellulaires entourées de deux couches concentriques de cellules renfermant de l'oléo-résine; mais ces glandes sunt surtout rapprochées de l'épiderme supérieur.

II. Nêreunc. - La nelvure biconvere est limitẻe par de l'épiderme muni de poils coniques unicellulaires et dont les cellules sont rectangulaires allongées suirant la longneur de la mervure. En dedans de l'épiclerme existe une masse de celfules ì membrane très épaissie surtout aux angles. Ces cellules sont polygonales en section transtersale: allongees, rectangulaires en section longitudinale. Quelques-unes renferment des agrglomérations de cristaux. Le nombre des couches qu'elles forment varie; il est plus grand it la face supérieure qu'ì la face inférieure. Entre ces deux masses parenchymateuses siège un cordon it section tranversale presque demi-circulare, composé inférieurement d'un arc libéro-ligneux très concare: supérienrement d'une bande également libéro-ligneuse réunissant les deus extrémités supérieures de l'arc inferieur, et d'une moelle occupant la concarité de l'arc. L'arc est constitué de mème que sa corde par du liber et du bois.

Le liber et composé, à sa partie erterne, de fibres à membrane cellulosique peu épaisse et ì cavité considérable ; à sa partie interne, de celIules dont quelques-unes renferment des cristaux açglomérés. 
Le bois, à peu près égal en puissance au liber, est formẻ de bandes rayonnantes vasculaires assez espacées les unes des autres et séparées par des rayons médullaires à deux ou trois rangs de cellules.

Quant à la moelle dont la section transversale est demi-circulaire. elle est composée d'éléments de deux sortes, ayant tous une membrane mince; les cellules voisines du bois sont petites et très allongẻes, tandis que les plus éloirnées sont plus larges, mais ont une hauteur moindre.

FAMILLE DES OXALIDËES.

Feuille doxalide oseille. - Oxalis acetosella. L.

Lame interneurale. a. Epiderme. - L'épiderme supérieur et l'épiderme inférlieur sont tous deux constitués pill une assise de grandes cellules incolores qui, examinées de face, présentent un contour sinueux. Observées d'après une coupe transversale de la feulle, elles paraissent rectangulaires; les parois, perpendiculaires ì lit surface, sont munies de ponctuations allongées.

Les deux épidermes sont ensemble pius épais que le mésophylle vert qu'ils recouvient (pl. IV, fig. VIII). Ils sont pourvus de poils unicellulaires; mais il n'y a de stomates qu'à la face inférleure. Les poils terminés en pointe à leur extrémité supérieure sont xétrécis à leur base : cette base est comprise entre trois ou quatre cellules épirdermiques.

La membrane des poils, un peu épaissie, est garnie à l'extérieur de nombreux tubercules arrondis.

Les stomates elliptiques sont placés au fond d'un petit canal prismatique ménagé entre trois ou quatre cellules de l'épiderme.

b. Mésophylle. - Contre l'épiderme supérieur s'appuie une couche de cellules coniques dont la base est superficielle. Ces éléments ont une hauteur au plus égale ì une fois et demie le diamètre de leur base. Ils lenferment presque tous de la chlorophylle; quelques-uns contiennent des cristaux agglomérés.

Au-dessous de cette couche existent deux ou trois assises parallèles de cellules, cellules qui, examinées en coupe transversale, paraissent rectangulaires; mais qui, vues de face, sont rameuses, et laissent entre elles de grands espaces polygonaux.

Nervire. - La nervure est petite. En haut et en bas elle est limitée par de l'épiderme. Les cellules épidermiques supérieures sont très rrandes, tandis que les éléments inférieurs sont très petits. Les deux épidermes, dépourvus de stomates, sont munis de poils semblables à ceux qui existent sur la lame.

Au-dessous de l'épiderme neural supérieur se trouve une couche de cellules vertes coniques qui se continuent de chaque côté avec le tissu vert supérieur du mésophylle. Au-dessus de l'épiderme inférieur est une assise de cellules incolores ayant la forme de prisme droit à base rectangulaire ou polygonale. Entre ce parenchyme et la conche cellulaire supérieure sière un petit amas de cellules cylindriques incolores ou renfermant quelques grains de chlorophylle. C'est dans cette masse que sont plongés de faibles cordons libéro-ligneux qui forment ensemble un arc dont la concavité est tournéc vers la face supérieure de la feuille. Chaque faiscenu est constitué par du liber et du bois. Les éléments du liber sont cellulosiques. Quant au bois, il est formé de quelques vaisseaux (trachées, vaisseaux spiraux). 


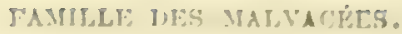

\author{
Guimauve oflicinale. - Athaca officinnlis. I.
}

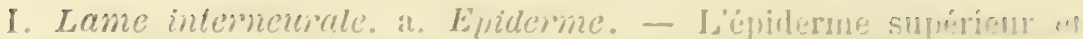
l'épiderme inféricur ont une seule conche de cellules tahbulanes, rectan.

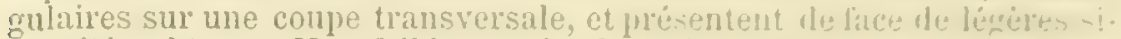
nuosités obtuses. Une fuible conche de cuticule les reconvere. ("es dr.ne: epidermes donnent maissance it des stomates, it des poils et it les glamlm.

Les stomates, disposés tris irrégulierement, sont elliptirnus.s.

Les poils (pl. III, fig. V, b) sont tous étoiles it folf luranches lonenter. droites, aigués au sommet et supportées par une base courte divisé par des cloisons longitudinales en autant de cellules qu'il y at de rancaux. Cette base, en forme de tronc de cône it rrande base supérieure, est percée de ponctuations arrondies et cntouréc cu grande partie par les cellules épidermiques.

Les glandes (a) sont pluricellulaires, sessiles ou munies d'un pédicelle. très-court. Elles sont généralentent oroides, il mosses extrémités libres. Elles sont formées de plusieurs rangées horizontales de cellules séparées par des cloisons verticales.

b. Mésophylle. - Le mésophylle pourvu de chloroplyylle est hétéro. gène. Le supérieur consiste en deux couches de cellules cylindrigues, it membrane mince, de deux a trois fois plus longues que laros. Lintérieur, à peu près égal au supérieur, forme de trois it cinq assises de petites cellules qui, sous une coupe transversale, paraissent irtégulières. assez rapprochées les unes des autres et sont dirigées tantút parallèlement, tantôt perpendiculairement ou obliquement par lapport it la surface foliaire. Examinées de face, elles semblent un peu rumeuses ì trois ou quatre branches. Les branches, quand il $y$ en a quatre. sont disposées en $\mathrm{X}$.

Dans ces deux parenchymes existent des cellules arrondies ou polyédriques contenant des cristaux agglomérés d'oxalate de chaux.

II. Nerrure. - La nervure, biconvexe, est limitée en laut et en bas par de l'épiderme dont les cellules trés allongées ont la for'me d'un prisme ¿ base carrée. Dépourvu de stomates, cet épiderme est muni de poils et de glandes commes celui de la lame.

Au-dessus de l'épiderme inférieur se trouve un collenchrme trés lléveloppé (pl. VII, fig. I, ll), comprenant de quatre à sept couches de longues cellules prismatiques ì base polygonale (de quatre it six côtés), dont les membranes présentent au point de contact des épaississements qui forment une converité dans l'intérieur de la cellule (collenchyme convexe).

Au-dessous de l'épiderme supérieur existe également du collenchyme convexe (i). C'est entre ces deux tissus que siege le parenchyme fondamental dans lequel est situé un arc libéro-ligneux, convexe inférieurement, et dont la concavité supérieure est remplie par des éléments cellulosiques.

Le parenchyme fondamental (a) est en majeure partie incolore. Le tissu vert de la lame pénètre très peu dans la nervure. Les cellules incolores a membrane mince, de deux it trois fois aussi longues que larges. sont rectangulaires en coupe longitudinale, et polygonales en coupe transversale; de plus, elles laissent entre elles des méats. Quelques cel. 
lules renferment des agglomirations dis cristaux d'oxalate de chaux (c).

L'arc libéro-ligneux est composé de liber, inférieurement; de bois, supérieurement.

Le liber (a) constitue plusieurs îlots séparés les uns des autres par deux files radiales de grandes cellules rectangulaires allongées de haut cn bas, dont quelques-unes renferment des cristaux. Chaque illot possède des fibres et des cellules Les fibres à parois un peu épaissies sont cellulosiques et ont une section polygonale. Quant aux cellules entrant dans la constitution du liber mou, elles forment un massif d'une puissance égale à celle du liber tibreux.

Le bois (b), concave a sa partie supérleure et un peu plus étendu que le liber, est constitué par plusieurs rangées de fibres, de vaisseaux rayés, spiraux et trachéens.

La concavité que laisse supérieurement le bois est occupé par un amas de cellules (e) à membranes épaissies ayant la forme de longs prismes droits à base irrégulièrement polygronale.

Mauve sauvage et Mauve à feuilles rondes. - Malva sylvestris. L. - Malva rotundifolia. $\mathrm{L}$.

La Mauve sauvage et la Maure ì feuilles rondes se distinguent de la Guimave en ce que les poils sont moins nombrenx. Tous ne sont pas étoilés. Quelques-uns sont simples et unicellulaires.

FAMILLE DES TERNSTRÉMIAEEES.

Fenille de Thé de Chine. - Thea chinensis. End.

I. Lame intemeurale a. Epiderme. - L'épiderme glabre, muni de stomates à la la face inférieure seulement. est formé d'une assise de cellules tabulaires ì contuur polygonal. Les parois de ces cellules très épaissies, sont recouvertes superficillement d'une couche épaisse de cuticule.

b. Mésophylle. - Le parenchyme vert constitue sur la face supérieure deux couches de cellules cylindriques pressées les unes contre les autres et allongées perpendiculairement à l'épiderme. Les superficielles sont en général de quatre à six fois plus longues que larges. A la face inférieure se trouve une lame aussi puissante que le parenchyme e:l palissade et composée de cellules, ¡ui, vues de face, sont rameuses, tiadis qu'en coupe transversale elles sont rectangulaires ou arrondies, et ne laissent entre elles que de faibles méats. Des cellules arrondies renfermant des cristaux agglomérés se rencontrent dans ce parenchyme méatique. Dans le mésophylle existent de longues cellules à membrane épaissie et lignifiée. Ces cellules partent pour la plupart de l'épiderme supérieur oì elles se fixent par une base épaisse assez étendue; de lì, elles parcourent le parenchyme dans son épaisseur, tantôt perpendicularirement, tantôt obliquenent, et doment naissance dans ce trajet ì des ramifications plus ou moins nombreuses (pl. IV, fig. IV, a).

III. Nervure. - Lil nervure est bi-convexe. Son épiderme, glabre et sans stomate, est composé d'une couche de cellules épaisses, tabulaires placées en file longitudinale et séparées les unes des autres par des 


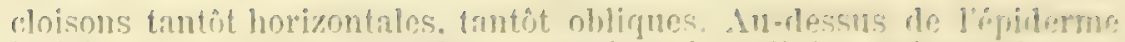
inférienr, se trouvent quelines conches de cellules prolyernales en

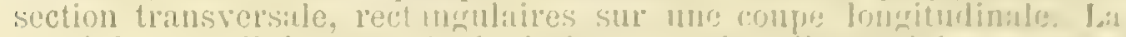

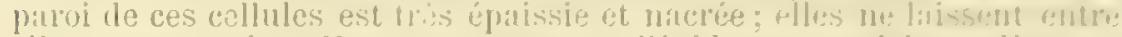

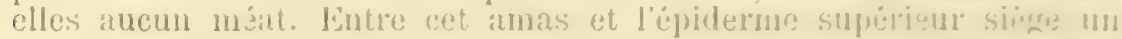
massif de ermoles cellules laissant entre felles des mótls. La contung de ces dernieres est polynonal: lit plupirt renfernent des mains de chlorophylle et quelques-unes contiennent des cristanx arghtomérés d'oxilate de chaux. Ce parenchyme est parcouru par" des sclérites semblables a ceux qui existent dans la limte, mais plus rameux. C'est au milieu de cet amas celluleux que se tronve le systeme libép-lignenx en forme d'arcassez étendu latéralement. Il est composö inférientement de liber qui est formé in sil base de denx couches continues de libres disposées en arc. Ces fibres ont une membrane épaisvie. linnjiée el ponctuce; leur cavité est assez considúrable. Au-dessus d'elles exist: un arc plus puissant que celui du liber fibreux; il est composé de cellules dont la plupart renferment des cristaux agromérés d'oralate de chaux. Le bois, deux fois plus développé que le liber, est constitué par des lames rayonnantes fibro-Tusculaires, séparées par des rayons médullaires trẻs aplatis et ì un senl rang de cellules. La concarité formée par l'are ligneux est occupée par un massil continu de fibres analogues i celles qui existent dans le liber inférieur.

FAMILLE DES EIYTIROXYLEES.

Feuille d'Erythroxylon coca. - Erythroxylon coca. L.

I. Lame interneurale. a. Epiderne. - L'épiderme, glabre. est pourru de stomates ì la face inférieure senlement. Une assise de cellules tabulaires, un peu épaissies, ì contour polygonal, le constitue. Les cellules de la face inférieure font à la surface une saillie proéminante en forme de cône à sommet très épaissi.

b. Mésophylle. - A la face supérieure existe une couche de cellules cylindriques disposées en palissade et en général de quatre it sis fois plus longues que larges. A la face inférieure, le parenchyme est composé d'une lame plus puissante que la couche de tissu en palissade. J.es éléments qui la constituent sont disposís en plusieurs couches parallèles. D'ordinaire, ils sont rectangulaires en coupe transrersale, et rameux it quatre branches disposées en H, lorsquon les examine de face. Le mésophylle ne donne point naissance it des cellules cristalligènes.

II. Marge. - La marge est constituée par la terminaison latérale du mésophylle et de l'épiderme.

III. Nerure. - La nervire est convere inférieurement. Les cellules de l'épiderme sont rectangulaires, allongées suivant l'axe de la nerrure, et ne laissent entre elles aucune ouverture stomatiques.

Contre l'épiderme s'appuie un amas de cellules à membrane épaissie et légèrement ponctuée. Polygonales en section transrersale, ces cellules sontrectangulaires sur une coupe longitudinale et levr grand coté est parallèle à l'axe de la nervure: quelques-unes l'entre elles renfer"ment des cristaux isolés d'oxalate de chaux. Le parenchyme inférieur. très développé, touche à un cordon médullo-libéro-ligneus, tandis qu'ì 
la face supérieure il en est séparé par une couche de cellules vertes se continuant latéralement avec le tissu en palissade de la lame. Le système cellulo-fibro-vasculaire se compose en allant de bas en haut:

$1^{0}$ D'ilots de fibres libériennes épaissies et lignifiées. Ces fibres ont un contour polygonal et leur cavité est assez grande Les îlots sont sẻparés par des cellules ponctuées, un peu épaissies.

20 De cellules libériennes formant un arc moins développé que l'arc fibreux.

30 D'un arc de bois plus puissant que le liber et composé de bandes rayonnantes de fibres et de vaisseaux, bandes séparées par des layons ì un seul rang de petites cellules.

40 D'un amas de cellules situć dans la concavité laissée supérieurement par le bois. Ces éléments ont une membrane épaissie, ponctuée et lignifiée. En coupe transversale, ils sont polygonaux, tandis qu'en coupe longitudinale, il sont rectangulaires, très allongés dans le sens de la nervire.

FAMILLE DES AUIRANTIACEES.

Oranger. - Citrus vulgaris. Riss.

I. Lame interneurale. a. Epiderme. - L'épiderme supérieur et l'épiderme inférieur, glabres, sont à une seule assise de cellules dont les membranes sont épaissies. Tabuliires ì surface irrégulièrement polyronale, ces surfaces sont recouvertes d'une conche épaisse de cuticule. L'épiderme inférieur seul est muni de stomates. Ces dernier's, elliptiques sont bordés latéralement par des cellules en forme de croissant dont la concavité est tommée vers les cellules stomatiques.

b. Mésophylle. - Le mósophylle forme is la partie supérieure deux couches de cellules vertes cylindri(pues (pl. IV, fig. II, c), pressées les unes contre les autres. Les superficielles, plus longues que les inféricires, sont de trois à quatr fois plus longues que larges. Ce parenchyme en palissade donne naissance in de grosses cellules arrondies voisines de l'épiderme et contenant chacune un seul cristal d'oxalate de chaux (b).

Dans le parenchyme inférieur (d), deux fois plus développé que le supérieur, les cellules sont faiblement rameuses (c); vues en coupe trunsversale, elles sont presque toutes elliptiques, allongées parallèlement ì la surface foliaire. On trouve dans cette masse et près de l'épiderme inférieur des cellules à cristaux isolés.

Le mésophylle contient de grosses glandes (a) arrondies multicellulailus. Ces glandes, voisines de l'épiderme, se trouvent presque toutes dans le parenchyme vert superieur.

II. Varge. - Il n'y a rien de particulier, si ce n'est la présence de grosses glandes semblables ì celles qui existent dans le parenchyme.

IIL. Nervure. - La nervure est bi-convexe. Son épiderme sans stomates a des cellules petites, carrées et polygonales, presque aussi longues que larges.

Au-dessus de l'épiderme inférieur vient une masse considérable de huit a dix assises de grandes cellules incolores, qui, sur une coupe transversale, sont polygonales ì membrane épaissie, surtout aux angles où l'épaississement parait triangulaire, et à cavité arondie ou légèrement polygonale. Sur une coupe longitudinale, elles paraissent rectan- 


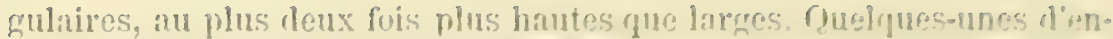
tre elles remerment des mistanx isoles.

Au-dessous de l'épiderine supérieur se trouvent de deux is duratre assises de cellules arondio: un peu épalissies ret ronfurunat de cratus de chlorophylle. Elles sont ovites, on romdes en conpe lingitminate. Ce parenchyme vert nenral se continue litéralement ave les colludes du

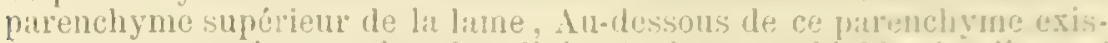
tent quatre on cinq couches de cellules epalsses semblibles it cellus roni sièrent au-dessus de l'épiderme infúrieur, nais beaucrup plus petites. Ce tissu donne naissance it des glandes analorrues il celles que l'on rencontre dans le mésophylle.

Le centre de lia nervure est ocenpée par un cordon qui, daus me sec. ton transverse a la forme d'une ellipse allongée paralliternent it la surface foliahe. Ce cordon est formé de deux ares libéro-lirneux dont l'un, intë. rieur, tourne en haut sa concavite, tandis que lintre, superieur, la tourne en bas.

Des cellules médullaires remplacont l'espace central laissé par ces deux ares.

Chaque arc libéro-ligneux est composé de liber et de bois.

Le liber est fibreux il sa surlace; les fibres, longues, limilites, tries épaissies, à section polygronale (5 côtés), ont une cavité intérieure faible. Elles forment des îlots entourant le liber mou aussi déreloppé que le liber fibreux.

Le bois, plus puissant que le liber, est constitué par un grand nombre de files rayonnantes de fibres très grosses et de viasseaux espacés. Cés files, séparées par des rayons médullaires très larges, sont trés rappro. chées.

Quant aux cellules médullaires, elles sont orales ou arrondies en section transversale et longitudinale. Elles sont aussi hautes que lar.res. épaissies, lignifiées, à cavité grande. Elles présentent sur toutes leurs membranes de nombreuses ponctuations. Quelques-unes il'entre elles contiennent des cristaux isolés d'oxalate de chanx; les autres contiennent des grains d'amidon.

FAMILLE DES TÉFÉBINTHACÉES.

Feuille de Sumac des corroyeurs. - Rhus coriaria. L.

I. Lame interneurale. - Jjépiderme muni de stomates à la face inférieure seulement, forme une couche de cellules tabulaires it contour polygonal ou légèrement simueux. Il donne naissance ai des poils cridnaires et à des poils glanduleux. Les poils ordinaires sont coniques. beaucoup plus longs que larges; un certain nombre sont pluricellulaires. Les poils glanduleux sont unicellulaires, courts, et ont ordinairement la forme d'un tronc de cone dont la grande base supporte directement une gldule en massue composée de deux ou plusietirs rangées horizontales de cellules sémarées par des cloisons verticales. L'épiderme est recourert d'une cuticule qui présente les crètes surtout ¿t la face supérieure. Le mésophylle vert constitue sur la face supérieure une conche de cellules cylindriques disposées en palissade, et en général de quatre à six fois plus longues que larges; ce parenchyme 
renferme des cellules qui contiennent des cristaux agglomérés d'oxalate de chaux. A la face inférieure existe une lame ordinairement de moitié moins développée que le parenchyme supérieur et composée de deux ou trois couches parallèles de cellules rectangulaires en coupe transversale, rameuses superficiellement; ce tissu produit des cellules cristalligènes.

II. Marge. - La terminaison latérale du mésophylle et de l'épiderme constitue la marge.

III. Nervure. - La nervure bi-convexe possède un épiderme dont les cellules a membrane épaissie ne laissent entre elles aucune ouverture stomatique. Cet épiderme est habituellement muni de poils coniques dont la longueur dépasse plusieurs fois la largeur, poils unicellulaires ou pluricellulaires avec leurs cellules disposées sur une seule rangée verticale. En deđans de l'épiderme siège, à la face supérieure et à la face inférieure, un amas de cellules qui ont la forme de longs prismes à base polygonale. Ces cellules qui ont la membrane épaissie, surtout aux angles, ne laissent entre elles aucun méat. Le collenchyme supérieur touche par sa couche profonde un faisceau constitué en partie par du liber et du bois, tandis que le collenchyme inférieur est séparé de ce dernier par un amas de cellules plus larges, mais moins hautes et moins épaisses. Un grand nombre de ces cellules renferment des cristaux agglomérés d'oxalate de chaux. Le système fibro-vasculaire est constitué par deux arcs, dont l'inférieur est assez considérable. Ces deux ares libéro-ligneux sont séparés au centre par un massif de petites cellules ayant la forme de prismes droits i base polygonale et contenant des cristaux agrolomérés d'oxalate de chaux. Chaque arcest composé de liber et de bois. Le liber, dont les éléments sont toujours cellulosiques, renferme de grands canaux limités par une ou deux couches concentriques de cellules contenant des huiles essentielles qui se déversent dans le canal sécréteur. Le bois est formé de plusieurs lames vasculaires rayonnantes séparées par des rayons médullaires minces à un seul rang de cellules.

Feuille du Sumac vénéneux. - Rhus toxicodendron. L.

Le Sumac vénéneux se distingue du précédent par plusieurs carac. tères. Il possède à la marge, au-dessous de l'épiderme, un faisceau de grandes cellules incolores, in membrane un peu épaissie. Leur nervure renferme à la lace inférieure plusieurs faisceaux libéro-ligneux très espacés, comme on le voit dans la figure III de la planche VII.

\section{FAMILLE DES LÉGUMINEUSES.}

Feuilles de Sénés. - 10 Casse à feuilles aiguës. - Cassia acutifolia. Guibourt.

I. Lame interneurale. a. Epiderme. - L'épiderme forme sur les deux faces une seule assise de cellules tabulaires dont le contour est polygonal. Il est pourvu de stomates et de poils aux deux faces. Les stomates elliptiques sont irrégulièrement disposés. La plupart d'entre eux sont situés sur la cloison transverse qui sépare deux petites cellules épidermiques. Leur ostiole allongée est dirigre pallallèlement it cette cloison. Les poils sont 
coniques, unicellulaires. Leur longueur depasse plusienrs fois la largeur. Leur base est enchassée jrofordérnent dans l'épiderne. Ils priesentent une paroi tres épaisse, munie de tuluercules it sa surfare: ils

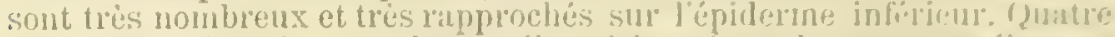
collules an plus séparent deux poils voisins. Quand cess appareils sont

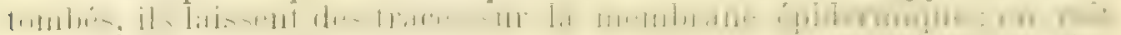
sur cetic dernière de petits polygones à côtés épais, entrurés de cellules rui rayonnent autour d'eux.

b. Mésophylle. - Le nésophylle constitue, al la face supérieure et is la face inferieure ( $\beta]$. IV, figr. Vi, $a, c)$, une couche de cellules eylindri. ques vertes et allongées perpendicularement it la surface do la feulle. Les cellules de la face supérieure sont beaucoup r.lus longrues que celles de l'infërieure; leur longueur dépasse de six à sept fois leur larment. tandis que les cellules inférienres sont de moitié ou du ticrs unoins longues. Entre ces deux couches en palissade existent quatre ou cinq conches parallèles de cellules plus yâles (b), le plus souvent rectangulaires, allongées parallèlement à lia surfice de la feuille. On rencontre dans ces dernières assises des cellules renfermant des cristaux agrinomérés d'oxalate de chaux.

II. Marge - Contre l'épiderme marorinal s'appuic un cordon de cellules pitles qui, sur une coupe transversale, ont un contour arrondi ou légèrement polygonal, tandis que, sur une coupe longitudinale, elles sont rectangulailes, allongées dans le sens de la nervure médiane; elles ne laissent entre elles aucun méat et leurs parois cellulosiques sont fuiblement épaissies, de manière ì Iaisser une rrande cavité cellulaire.

III. Nerure. La nervure est convexe inférieurement. Son épiderme, sans stomates et pourvu de poils analogues à ceur qui existent sur la lame, est composé de cellules en forme de prismes droits à base carrée. Au-dessus de l'épiderne infériemr se trouvent plusieur's courches de cellules páles, faiblement épaissies; la surface transversale de ces cellules est polygonale, tandis qu'elle est rectangulaire sur une section longitudinale. Les cellules supérieures de ce massif, presque aussi larires que hautes. touchent un cordon libéro-ligneux et renferment des cristaux isolés d'oxalate de chaux. Au-dessus de l'épider'me supérieur existent. au contraire, des cellules remplies de grains de chlorophylle, cylindriques, assez serrées et perpendiculaires à l'épiderme; ce tissu rert se continue latéralement avec le parenchyme en palissade supérieur de la lame. Entre ce dernier et le par'enchyme incolore inférieur siège un cordon dont la section transversale est elliptique ì grand diamètre latéral. A sa partie inférieure, ce cordon est constitué par deux ì quatre couches continues de fibres à contour polygonal, à carité assez considérable, à membrane assez épaissie, ponctuée et lignifiée. Au-dessous du prosenchyme libérien viennent les cellules du liber formant par leur ensemble un are égal ou inférieur à larc fibreux. Le bois placé à la partie supérieure du liber est composé de bandes rayonnantes de quatre á six vais. seaux chacune et séparées les unes des autres par des rayous médullaires composés de une ou deux rangées de cellules jamaîs lignifiées. Au-dessus du bois siègent quelques couches de cellules à membranes lignifiées et ponctuées formant une moelle; les cellules supérieures touchent un massif de fibres semblables ì celles du liber inférieur. 
$2^{\circ}$ Casse à feuilles étroites. - Cassia angustifolia. Vahl.

Le Cassia angristifolia se distingue du précédent par ses poils moins nombreux sur l'épiderme supérieur', poils dont les plus rapprochés sont séparés les uns des autres par six à dix cellules, tandis que dans le Cassia acretifolia il ne sont séparés que par deux ou quatre cellules.

3o Casse à feuilles obovées. - Cassia obovata. Colladon.

Dans le Cassia oborala, les cellules de l'épiderme inférieur forment ì la surface des saillies coniques. L'épiderme donne naissance à des glandes ovoïdes, unicellulaires dont la grosse extrémité s'insère sur un pédicelle unicellulaire très court. Le mésophylle ne produit point de cellules cristalligènes.

Falsifications. - On peut rencontrer dans les feuilles de Séné différentes espèces dont la plus commune est l'Arguel (Solenostema Ar. guel, de la famille des Asclépiadès). On y trouve des feuilles de Redoul (Coriaria myrtifolia, famille des Coriacées), de Baguenaudier (Coluten amborescens), et de Théphrosie (Thephrosia apollinea).

LAirguel se distingue facilement des Sénés par son mésophylle et sa nervure. Le mésophylle présente, comme dans le Séné, du tissu en palissade ì la face supérieure et inférieure de la feuille; seulement il forme it ces endroits plusieurs assises superposées.

Le faisceau libéro-ligneux contient à sa face supérieure et inférieure des îlots de liber qui ne présentent jamais de fibres lignifiées.

Le mésophylle du Redoul est hétérogène asymétrique. Son cordon libéro-vasculaire est entouré đ'une couche de cellules différentes des éléments environnants, couche lui formant une gaine. Son liber ne renferme point de fibres lignifiées. Dans aucune partie de la feuille on ne trouve de cellules renfermant des cristaux d'oxalate de chaux; de plus il n'y a point de poils à l'épiderme.

Le Baguenaudier se distingue sans peine des Sénés. L'épiderme possède des cellules qui ont un contour sinueux; il est muni de poils unicellulaires rétrécis à leur base, et les membranes de ces poils ne sont point tuberculeuses.

Le mésophylle hétérogène asymétrique constitue ì la face supérieuro denx ou trois conches de tiscul muriforme.

Dans le Thephrosia, le mésophylle est hétérogène symétrique; mais le tissu en palissade forme aux deux faces plusieurs couches de cellules. Il n'y a pas de parenchyme muriforme vert à la partie supérieure de la nervure; mais il existe, au-dessus du faisceau, un petit massif dont la section transverse est quadrangulaire et dont les éléments incolores et polygonaux ont les parois un peu épaissies.

Thephrosia apollinea. D. C.

I. Lame intemenrale. a. Epiderme. - L'épiderme constitue sur les deux faces de la feuille une assise de cellules aplaties dont le contour superficiel est irrégulièrement polygonal. Les deux épidermes portent des poils, mais l'inférieur seul est pourvu de stomates.

Les poils sont couchés, coniques, uni ou bicellulaires. Dans ce dernier 
cas, in cellule basilare est tris pen élerée, tandis que la superienre ose

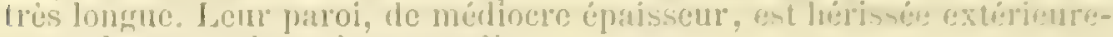
ment de wos tubercules arrondis.

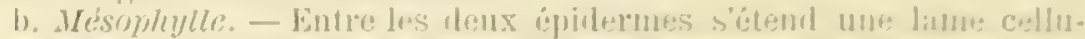
laire dont les éléments renferment de la chlomphylle. Joux ou toin conches de cellules cylindrignes, perpendiculates it lat surface de lat leuille, s’appuient contre l'épiderne inférieur et l'épiderme supéribur. Ces deux parenchymes en parlissade sont séparés par deux ou trois assises de cellules aplaties de haut en bas, mais allongres paralleternent it la surfitce foliaire.

II. Nervure. - L'épiderme neural est recouvert de poils ofliant les mêmes caractères que ceux de la lame. Ses cellules, eximinces de face. ont la formes de rectangles dont les grands cutés sont dirigés daus le sens longitudinal de la nervure. Dans l'intérieur de celle-ci est un cordon dont la section transversale a l'aspect d'un triangle dont la base courbe est inférieure, tandis que le sommet est tourné vers la partie supérieure. On trouve de la base au sommet de ce triangle:

$1^{\circ}$ Un are continu de fibres libériennes qui sont polyronales sur une coupe transver'sale. Leur paroi épaissie est ligniliée:

20 Un arc de liber mou :

30 Du bois composé de plusieurs rangées parallèles inféro-supérieurés de valisseaux ponctués, spiraux et de trachẻes:

$4^{\circ}$ O'un amas de cellules ayant la forme de prismes droit at base polygonale et possédant des membranes épaissies et lignifiées.

$5^{\circ}$ D'un triangle de fibres semblables il celles qui existent it lit face inférienue du cordon.

Dans certains cas, on remarque au-dessus de la masse cellulaire située ¿l la face supérieur du bois, un petit groupe libéro-ligneux tournant sont liber vers la partie supérieure de la nervure.

Le cordon est en contact inférieurement avec du parenchyme dont les éléments, disposés en files longitudinales, laissent entre eux des méats. Ces cellules ont l'aspect de gros prismes droits il base polyonale. Celles qui touchent le faisceau renferment chacune un cristal doxalate de chanx.

Entre l'épiderme supérieur et le sommet du triancle se troure un petit massif dont la section transrersale est quadrangulaire. Les éléments ı ui le constituent sont prismatiques et incolores.

Les feuilles de cette plantes peuvent être confondues arec celles de Séné oboré.

\section{Feuille de Baguenaudier arbuste. - Colutea arborescens. L.}

I. Lame internenrale.-L'épiderme, pourvu de stomates sur ses deux faces, est composé d'une assise de cellules tabulaires et à contour légèvement sinueux; les stomates elliptiques sont situés entre quatre ou six cellules de l'ẻpiderme. L'épiderme donne naissance, à la face inférieure, i des poils coniques, unicellulaires, aigus ì leur extrémité et présentant une longueur qui dépasse plusieurs fois leur largeur; leur base, faiblement enchâssée dans l'épiderme, a un diamètre toujour's moindre que le diamètre pris à la moitié de sa longueur. Leur paroi un peu ẻpaissie est chargée de petits tubercules (pl. III, fig. VI.

Le mésophylle vert forme ì la face supérieure deus ou trois couches 
de cellules cylindriques disposées en pulissade; les superficielles sont trois ou quatre fois plus longtres que larges. A la face infériemre existe une lame cellulaire moins développée que le parenchyme supérieur: les cellules qui lit composent ont un contomr sinueux quand on les voit sur une coupe transversale, tandis que de face, elles sont arrondies ou polygonales. Les plus voisines de la face inférieure sont allongées perpendiculairement à l'épiderme.

II. Marge. - La marge est uniquement constituée par la terminaison latérale du mésophylle et de l'épiderme.

III. Nervure. - Lépiderme, un peu poilu et dépourvu de stomates, est composé d'une couche de cellules ayant la forme d'un prisme droit i base quadrangulaire; les parois de ces cellules sont épaissies et lécrèrement ponctuées. En dedans de l'épiderme existe un massif de cellules épaissies, cellulosiques, qui présentent de légères ponctuations; elles ont un contour polygonal et sont rectangulaires sur une coupe longitudinale. Le massif situé ì la face supérieure est moins étendu latéralement que l'inférieur; en section transtersale, il a la forme d'un rectangle à grand côté perpendiculaire ì la surface de la feuille. Entre le parenchyme supérieur et le parenchyme inférieur, siège un cordon dont la section transversale est triangulaire, à sommet supérieur et à base inférieure; il est composé en allant de bas en haut: $1^{\circ}$ de liber fibreux; $2^{\circ}$ de liber celluleux; $3^{\circ}$ de bois ; $4^{\circ}$ de cellules épaissies.

Les fibres du liber forment trois ou quatre couches continues disposées en arc; elles ont un contour arrondi ou légèrement polygonal; lem" membrane épaissie et lignifiée enveloppe une cavité assez considérable. Le liber celluleux, aussi développé que le fibreux, est disposés en îlots séparés par un ou deux rangs de cellules assez larges. Le bois est forme de quelques lames rayonnantes de deux à six vaisseaux assez larges. Quant à la moelle, elle forme un petit amas de cellules épaissies et céllulosiques dont la section transverse est polygonale.

Cette feuille peut être substituée au Séné.

FAMILLE DES ROSACÉES.

Espèces: Ronce arbrisseau. Rubus fmuticosus. L. - Laurier cerise. Cerasus laurocerasus. L. - Pècher. Persica rulgaris. L. - Alchemille commune. Alchemilla vulgaris. L.

I. Lame interneurale. a. Epideme. - L'épiderme constitue sur les deux faces une couche de cellules tabulaires dont le contour est polygonal, (Laurier cerise et Pêcher), ou sinueux (Alchemille et Ronce). Les deux épidermes sont pourvus de stomates dans l'Alchemille, tandis qu'il n'existe de ces appareils qu'ì la face inférieure dans les trois autres espèces. L'épiderme est glabre (Ronce, Pêcher, Alchemille) ou poilu (Ronce). Les poils de cette dernière plante sont étoilés.

b. Mésopłılle. - Le mésophylle est hétérogène symétrique et produit des cellules cristalligènes. Le tissu en palissade forme généralement deux ou plusieurs issises.

II. Marge, - Le bord foliaire est constitué par du parenchyme vert recouvert d'épider'me dans le Pècher, la Ronce et l'Alchemille.

Le Laurier cerise présente contre l'épiderme quelques cellules incolores à membrane épaissie.

III. Nervure. - L'épiderme neural, glabre dans le Pêcher et le Lau- 
rier cerise, est poilu dans les deux antres espoices. Iyans l'A lehernille los poils sont lones, epaissis, unicellulaires. Dins la lence, ils sont les uns simples unicellnlatios, les autres itoiles.

Le systime libéro-ligneux est represchte par un aro dont lit concavive est tournée vers la face supericure de la feuille. Dins l'A fehemillo les deux extrémités de ce croissant sont tris rappruchées. Lo libor bentom-

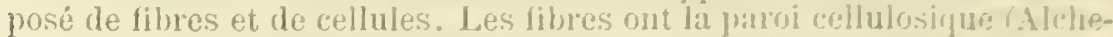
mille, P'ècher) ou lignifiée (Ronce, Lanuel cerise). Le bois est componé de bandes vasculaires parallèles et rérulieres.

Le systeme fibro-rasculaire est plongé dans une masse cellulaire, dont les éléments, assez volumineux, ont la paroi plus ou moins épaisse. Quelgues-uns d'entre eux renferment des cristaux d'oxalute de chatux. Co parenchyme est d'ordintire on contact avec des cellutes épaissies qui forment sur les deux faces un amas touchant lépiderne.

Le faisceau de Thehemille est cntoure d'ume couche de cellules aplatie constituan une sorte d'assise protectrice.

Feuille l'Alchemille commune. - Alchomilla rulgaris. L.

I. Lame intencurale - Lépiderme, glabre et pourvu de stomates aux deux faces, est constitué par une assise de cellules tabulaires minces ì contour sinueux. Le mésophylle, dépourvu de cellules cristal. lirènes, forme à la face supérieure une couche de cellules cylindriques. allongées perpendiculairement it la surface de la feuille et de trois it quatre fois plus longues que larges. A la face inférieure existe une lame en grenéral égale en puissance au parenchyme supérieur et composée de cellules rameuses laissant entre elles des méats plus ou moins considérables.

II. Marge. - La marge est uniquement constituée par de l'épiderme recouvrant la terminaison latérale du mésophylle.

III. Nervure. - Les cellules épidermiques de la nervure, généralement rectangulaires lorsqu'on les examine de face et allongées paralle. lement it l'axe de la nerrure, ont une membrane un peu épaissie. ponetuée. L'épiderme donne maissance a de longs poils coniques, unicellulaires, dont la yaroi est un peu épaissie; la base des poils est enchàssée dans l'épiclerme. En dedans du tissu épidermique sière un amas de grandes cellules qui ont la forme d'un cylindre ou d'un prisme droit i base polygonale; ces cellules, à membianes un peu épaissies et ponctuées, sont pàles et quelques-unes renferment des cristaux agcrlomérés d'oxalate de chaux. C'est dans cette masse que se rouve un cordon dont la section transverse est elliptique, à grand diamétre dirigé de bas en haut; ce cordon ne touche pas immédiatement les grandes cellules incolores du parenchyme fondamental, il en est séparé par une couche de cellules petites, qui, sur une coupe transtersale paraissent allongées tangentiellement au faisceau. Le coldon est parfois composé, d'une part, d'un arc inférieur trés recourbé et dont les extrémités supérieures sont assez rapprochées; d'antre part, d'une courte lame réunissant les deux extrémités de cet arc; quelquefois cette bande rectiligne libéro-ligneuse n'existe point; dans tous les cas, la concavité de l'are est occupée par un amas de cellules jouant le rôle de moelle. Le liber,

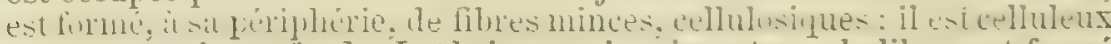
dans sa partie profonde. Le bois, aussi puissant que le liber. est formé 
d'un grand nombre de lames rẻgulières et rayonnantes de plusieurs vaisseaux; ces lames vasculaires radiales, très rapprochées, ne sont séparées les unes des autres que par un senl rang de petites cellules.

La moelle est composée de cellules qui ne laissent entre elles aucun méat. Ces éléments ì membrane épaissie, surtout aux angles, ont la forme de longs prismes droits à base polygonale.

Feuille de Lauricr cerise. - Cerasus lauro cerasus. L.

I. Lame intemeurale. a. Epiderme inférieur. - L'épiderme inférieur possède des éléments rectangulaires allongés superficiellement, quand ils sont vus en coupe transversale; et polygonaux, carrés, sans sinuosités, si onl les examine de fíce. Ces éléments ont une membrane assez épaisse et ponctuée. Ils sont en outre recouverts à leur surface d'une couche épaissie de cuticule. Les stomates, arrondis, sont limités latẻralement par deux cellules en croissant.

b. Epiderme supéricur. - Analogue à l'inférieur, il possède cepelldant des cellules en général plus grandes; de plus, on ne remarque point de stomates.

c. Mésophylle. - Le mésophylle situé entre les deux couches épidermiques n'est point semblable aux deux faces. Sous l'épiderme supérieure, les cellules, perpendiculairement dirigées à la surface foliaire, sont pressées les unes contre les autres. Elles sont cylindriques et ont une longueur qui, dans les plus grosses, dépasse à peine cinq fois la lingeur; de deux à quatre couches de ces éléments constituent le parenchyme en palissade. Le parenchyme inférieur a de grosses cellules rameuses, laissant entre elles des lacunes plus ou moins considérables; sur une coupe transversale, elles paraissent orales, elliptiques ou l'ectangulaires dirigées en tout sens. Ce dernier parenchyme a une épaisseur un peu plus grande que celle du parenchyme supérieur.

Les cellules de ces deux parenchymes contiennent de la chlorphylle; quelques-unes cependant, plus grosses en général que les éléments quí les entourent, renferment de gros cristaux agglomérés d'oxalate de chaux.

II. Marge. - L'épiderme de la marge ne diffère guère de celui de la lame proprement dite, mais contre cet épiderme sappuie de une à trois couches de cellules épaissies, ponctuées, arrondies tranversalement, rectangulaires dans le sens longitudinal. C'est contre ce massif de renforcement que vient se terminer le parenchyme rert.

III. Nervure. - L'épiderme supérıeur est semblable à l'épiderme supérieur de la lame, l'épiderme inférieur a les cellules carrées ou rectangulaires (vues de face), et allongées suivant la nerrure. On ne trouve point de stomates.

Directement, contre les deux épider'mes, on rencontre de l'hypoderme cellulosique dont les cellules varient de grandeur et d'étendue suivant qu'on l'examine à la face inférieure ou it la face supérieure. L'hypoderme inférieur est composé de cinq à huit couches cellulaires occupant toute convexité de la nervure. Les cellules, arrondies ou lécèrement polygonales sur une coupe transversale, rectangulaire et allongée, dans une coupe longitudinale, ont des parois très épaisses et munies de nombreuses ponctuations. Parmi ces cellules, qui ne laissent entre elles aucun méat, il y en a qui renferment dans leur intérieur de gros cristaux d'oxalate de chaus. 
L'hyporerme superieur: moins itendu lateralement que l'infétieur. possede des éléments beancoup plus gros.

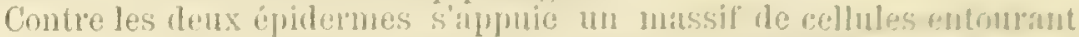

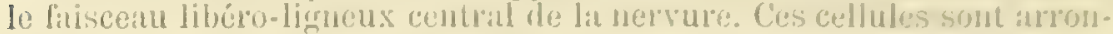
dies ou polygonales, vues en coupe transversile; rectangulaties no carrées sur une coupe longitudinale; olles juésentent des ponctuations et ne laissent entre elles aucun méat. Elles ne renferunent point de chlorophylle.

Faiscear bibéro-ligneux. - Le systeme libéro-ligneux at la forme d'un are convexe inféricurement. Il est constitué par du liber et du bois. Lf liber. inférieur par rapport au bois, est composé de fibres et de cellules. Les fibres ì parois cellulosiques sont disposées en ilots irréruliers séparé: les uns des antres par une série radiale de larese cellules arrondiess ru polygonales sous une coupe transversale; rectangulaires, ponctuées en coupe longitudinale. Ces fibres à section polygronale ont une une mem. brane épaisse, blanchatre, et laissent au centre une assez large ourerture de forme tres irreguliere; de plus, on remarque toujours au milieu de ces fibres des cellules contenant des masses cristallines dioxalate de chaux. Au-dessus du liber vient le bois constitué inférieurement par deux ou trois couches de fibres allongées, ponctuées, trés petites, it membranes très épaissies laissant une lumière car'ée relativement considérable. Au-dessus des couches fibreuses se remarquent les puissantes séries radiales de vaisseaux, rayés, ponctués, spiraux et trachéens dont les ouvertures sont plus larges et les membranes moins épaisses que celles des fibres ligneuses.

Dans la concavité que forme le bois se trourent quatre ou cinq couches de cellules à membranes épaisses. Ces cellules, qui forment une espèce de moelle, sont circulaires ou orales sur une coupe transversale; rectangulaires longitudinalement. Elles possèdent de nombreuses ponctuations.

Feulles de Pècher commun. - Persica rulgaris. Mill.

I. Lame intemeurale. a. Epiderme. - L'épiderme, glabre, est formé d'une assise de cellules tabulailes dont le contour est polyconal. L'infé rieur seul est pourvu de stomates elliptiques placés entre plusieur's cellules épidermiques.

b. Mésophylle. - Il existe entre les deur épidermes du parenchyme :n palissade et du tissu méatique. Le premier est composé de deur couches de cellules cylindriques dont les supérieures sont de cing it sept fois plus longues que larges. Au voisinage de l'épiderme et entre les cellules vertes se trouvent des éléments sphériques et incolores qui renferment des cristaux agglomérés d'oxalate de chaux. Au-dessous de ces deux assises on remarque des cellules vertes irrégulières et diriquées en tout sens. Des éléments cristalligènes sont disséminés aians ce parenchyme.

II. Nervure. - La nervure offre de bas en haut:

$1^{0}$ Une couche d'épiderme dont les cellules aplaties perpendiculairement ì l'are sont allongées dans le sens de la longueur de la nervure et présentant des parois un peu épaissies et ponctuées:

$2{ }^{\circ}$ De deux it quatre assises de cellules cylindriques dont les membranes natrés. épatissies et cellulosiyues. sont munies de ponctuations; 
$3^{\circ}$ Une massc de grandes cellules qui ont la forme de prisme $\mathfrak{i}$ base polygonale et qui sont moins élevées que les cellules précédentes; en outre, leur's enveloppes sont minces, et elles laissent entre elles de petits méats. Quelques-unes, disposées en files longitudinales, contiennent des agglomérations cristallines;

$4^{0}$ De nombreux îlots libériens dont les éléments possèdent une membrane qui offre les réactions de la cellulose. La plupart de ces éléments renferment des matières albuminoïdes: mais, au milieu de ces derniers se trouvent des rangées cellulaires longitudinales dont chaque cellule contient des cristaux ;

$5^{\circ}$ Un arc ligneux composé de nombreuses lames parallèles de gros vaisseaux:

$6^{\circ}$ Un parenchyme dont les cellules sont petites, et ont la forme de prismes a bolygonale;

7. Un massif de grandes cellules qui ont une membrane mince et laissent entre elles des méats. Un certain nombre possèdent dans leur intérieur des cristaux agglomérés;

8. Des éléments cylindriques épaissis et ponctués;

$9^{\circ}$ Une couche d'épiderme supérieur.

Ronce arbrisseau. - Rubus fruticosus. L.

I. Lame intemenrale. - L'épiderme, pourvu de stomates à la face inférieure seulement, est composé d'une assise de cellules tabulaire à contour sinueux, dont les membranes un peu épaissies et ponctuées sont revêtues d'une couche de cuticule assez épaisse. L'épiderme produit des poils courts et étoilés; les branches de ces poils sont supportées par un pédicule dont la base est enfoncée dans l'épiderme. Le mésophylle vert forme à la face supérieure deux conches de cellules cylindriques disposées en palissade; les plus superficielles sont en en général de trois à cing fois plus longues que larges. On remarque dans ce parenchyme quellques cellules renfermant des cristaux agglomérés d'oxalate de chaux.

A la face inférieure se trouvent deux ou trois couches parallèles de cellules qui sont en général rectangulaires sur une coupe tranșversale, tandis qu'elles sont rameuses et qu'elles laissent des méats superficiellement. Toutes les cellules du mésophylle contiennent du tannín.

II. Marge. - La marge est formée par la terminaison latérale du mésophylle et de l'épiderme.

III. Nervure. - La nervure, convexe inférieurement, est limitée en haut et en bas par de l'épiderme dépour'vu de stomates et composé d'une couche de cellules allongées parallèlement à l'axe de la nervure, cellules dont la membrane épaissie présente des ponctuations. Cet épiderme donne naissance à des poils de deux sortes: les uns sont simples, unicellulaires, longs, à membrane extrêmement épaisse; les autres, étoilẻs, ressemblent à ceux de la lame.

En dedans de l'épiderme existent quelques couches de cellules pâles ayant la forme de longs prismes à base polygonale; ces cellules, à membrane nacrée, très épaisse surtout aux angles, ont une cavité assez considérable et ne laissent entre elles aucun méat. Contre cet hypo. derme s'appuie un massif de cellules beaucoup plus larges, mais moins hautes, et dont les parois moins épaissies présentent des ponctuations allongées perpendiculairement aux arêtes; quelques-uns d'entre elles 
contiennent res cristanx acrelonobles dioxalate de chnux. Co massif

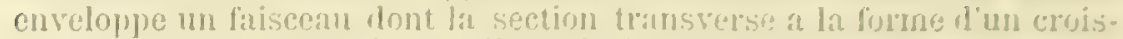

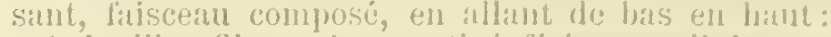

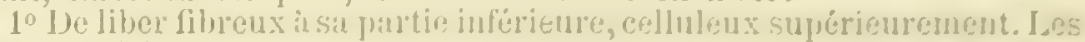

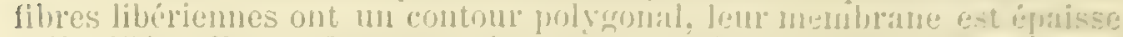

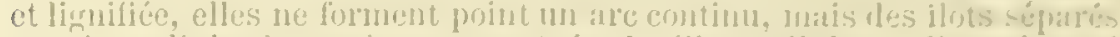

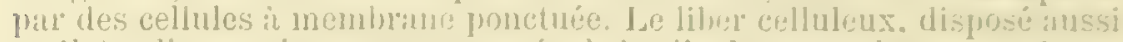

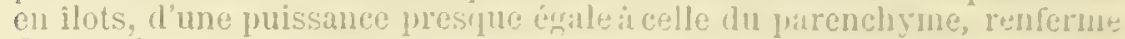
du tannin;

$2^{\circ}$ D'un arc de bois composé d'un gland nonbre de bandes fibro-vas. culaites rayonnantes et séparécs les mes des autres pall un scul l'ang de petites cellules:

$3^{\circ}$ D'un amas de petites cellules gui ne laissent entre elles aucun meat et ont lia forme de longs prismes droits it base polyronale; leurs membranes sont épaissies et pouctuécs.

FAMILLE IDES MYRTACEES.

Eucalypte globuleux. - Eucalypeus glouulus. Isaljillardičre (1).

I. Lame inteinenale. a. Epiderne. - L’épiderme supérieur et l’épiderme inférieur n'ont gu'une seule assise de cellules tabulaires it surface polyédrique irrégulière. Ces cellules ont une paroi épaissic et sont recouvertes à leur surface d'unc couche assez épraisse. Les deux épidermes sont glabres et possèdent des stomates arrondis clisposés sans ordre.

b. Mésophylle. - Le mésophylle est formú de deux sortes de cellules vertes. Les cellules roisines des deux épidermes (pl. IV, fig. 11 , a, c), sont cylindriques, pressées les unes contre les autres et allongres perpendiculairement ì la surface de lépiderme; elles forment sur chaque face deux ou trois couches de cellules. Dans lat lame intermédiaire. les cellules sont un peu irrécrulières, la plupart allongrées perpendiculaire. ment it la surface foliaire et laissant entre elles de petits méats (b). Des cellules renfermant des cristaux d'oxalate de chaux ic), les uns isolés, les autres agglomérés, et de grosses glandes multicellulaires spériques (d), contenant une huile essentielle jaunitre, se rencontrent dans le mésophylle.

II. Marge. - Tout contre lépiderme marginal siège un faisceau de cellules à membranes épaisses, ponctuées et cellulosiques.

III. Nervure. - La nervure est limitée en haut et en bas par des cellules épidermiques polygonales, épaisses et couvertes d'une coucle épaisse de cuticule. Contre ces deux ćpidermes se troure une masse de grandes cellules à membrane épaissie, arrondie ou légèrement polygonale en section transversale, rectangulaire et quatre fois plus hate que large en coupe longitudinale; quclques-unes de ces cellules contiennent des cristaux isolés d'oxalate de chanx. Dans ces deux masses de cellules siège un cordon de forme elliptique en section transversale, cordon composé d'un arc libéro-ligneux et de deux arces supérieurs roisins. Les arcs supérieurs ne se touchent pas au centre; ils laissent un faible

(1) Voir l'étude de M. de Lanessan sur les feuilles d'Eucalyptus globulus Lab. en Histoire des Drogues de Fluckiger, t. I, page 51.5. 
espace occupé par de la moelle. Chaque arc est constitué par du liber externe, du bois et du liber interne.

Le liber externe est fibreux en mème temps que celluleux. Les fibres, lignifiées, longues, à membrane très épaissie et à cavité faible, forment des îlots ì la limite externe du liber mou qui, lui, est composé de cellules grillagées, séveuses et cristalligènes.

Le bois est constitué par plusieur's files régulières et rayonnantes de vaisseaux et de fibres; ces files sont très rapprochées et séparées par un rayon médullaire i un seul rang de petites cellules épaissies. Le bois est i peu près aussi puissant que le liber externe.

Le liber interne, moins développé que l'externe. ne possède, outre les cellules semblables à celles de l'externe, que quelques fibres isolées.

La moelle, assez restreinte, a des cellules épaissies, polygonales et très peu élevées; un grand nombre renferme des cristaux d'oxalate de chaux.

Feuille de Myrte, - Myrtus communis. L.?

Lame intemerrale a. Epiderme. - Les cellules épidermiques sont aplaties et présentent des parois sinueuses; elles sont recouvertes d'une couche de cuticule très épaisse. L'épiderme inférieur offre seul des sto. mates qui affectent des dispositions irrégulières.

b. Mésophylle. - Au-dessous de l'épiderme supérieur sont deux assises de parenchyme vert en palissade dont les éléments n'ont point la mème longueur. Entre ce tissu et l'épiderme inférieur est comprise une lame cellulaire qui a une épaisseur au moins deux fois plus grande que le parenchyme en palissade; ses cellules ont une forme assez variée et laissent entre elles des méats considérables.

On rencontre dans le mésophylle des cellules incolores qui contiennent des cristaux agglomérés d'oxalate de chaux et des glandes pluricellu. laires sphériques. Ces dernières siêgent dans la portion de tissu qui avoisine l'épiderme.

Nervure. - Au milieu de la nervure chemine un faisceau libéro-ligneux formé de liber inférieur ou externe, de bois et de liber supérieur ou interne.

Le liber externe et le liber interne ont la mème constitution anatomique : ils sont composé de fibres disposées en îlots et entourées d'une membrane très épaissie, ponctuée et lignifiée ainsi que d'un amas assez puissant de liber celluleux qui n'offre point de cellules cristalligènes.

Le bois présente des trachées et des vaisseaux ponctués rangés en bandes rayonnantes.

Entre le système fasciculaire et l'épiderme supérieur, se remarque un massif hypodermique de longues cellules incolores prismatiques dont la section transversale est circulaire ou irrégulièrement polygonale. La paroi de ces éléments est assez épaissie et offre les réactions de la cellulose. Quelques cellules de ce tissu renterment chacune de volumineux groupes de cristaux. Un tel hypoderme existe aussi au-dessus de l'épiderme inférieur.

L'hypoderme inférieur touche par sa face supérieure un parenchyme formé de cellules plus courtes, mais plus larges, ayant l'aspect de pris. mes droits a base polygonale. On rencontre dans quelques-uns de ces

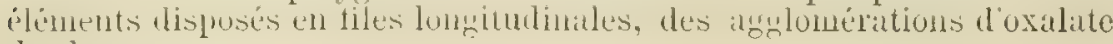
de chaux. 
Falsificalions. - On pent confondre les fonilles de linis (liumes sem-

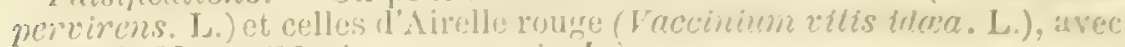
colles de Myrte (Myrtus commumis.L.)

Voici les carictires principaux qut permettront de les distingurer.

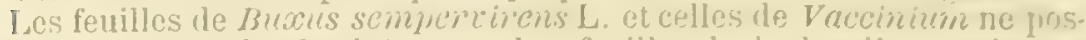
sedrent point de glandes internes. Jes fenilles de lat derniere respocer me prosentent point de cellules cristalligunes, mais ollient at leur borl an corklon de fibres limifices.

Le systëme fondamental de la nervure du buxus semperirens.L. est composé de cellules vertes semblables à celles qui existent dans le mésophylle extra-neural.

FAMILLE DES GROSSLLARIFES.

Feuille de Groseillier noir. - Ribes nigrum. L.

I. Lame interneurale. a. Eviderine. - L'épiderme est formé d'une assise de cellules tabulaires i contour sinueux. Il est recourert l'une couche mince de cuticule. L'épiderue inférieur est muni de stomates et donne nitssance d des rlandes rolmmineuses. Colles-ci, discoides, sont composées de quelques rangées paralleles et horizontales de nombreuses cellules, produisant de l'huile essentielle qui souleve la cuticule et s'interpose entre cette dernière et les éléments sécréteurs (pl. II, fing. II).

b. Mésophylle. - Le parenchrme en palissade est composé d'ume couche de cellules cylindriques, perpendiculaires à la surface de la ferille et de quatro à cinq fois plus longues que larges. Au-dessous de lui existent quelques assises de cellules ilrégulières et rertes. au milieu desquelles se trouvent de raves ćments cristalligúnes. Ce tissu lacuneux est égal en puissance au parenclıyme supélieur.

II. Nervore. - L'épiderme neural a les éléments allongés suirant l'axe, et rectangulaires quand ils sont rus de fice. Il possede des stomates et produit d'assez lonrs poils unicellulaires coniques dont les membranes sont munies de tubercules a leur surface. Deux à trois coude collenchyme sont adossées it l'épiderue. Entre le collenchyme supérieur el le parenchyme inférieur sière un amas de cellules assez grandes qui ont la forme de polygone (coupe transversale) ct possedent des pa. rois assez minces; quelques-unes lonferment des cristaux agglomér és d'oxalate de chaux.

Ce tissu enveloppe un cordon composé d'arc libéro-ligneux concare. et d'une masse cellulaire remplissant la concarité que forma le bois supérieurement.

Le liber est constitué inférourement par un åc continu de fibres dont les parois sont minces et cellulosiques; supérieurentent par les ilots celIulaires.

Le bois est composé de plusieurs bandes rayonnantes de vaisseaux, bandes séparées par deux rangées de cellules.

Les éléments qui entrent dans la constitution du parenchrme supérieur ont la forme de longs prismes dont la base est polygonale. Leur paroi, épaissie aux angles, est ponctuée et cellulosique. 
FAMILLE DES ARALIACEES.

Lierre grimpant. - Hedera helix. L.

I. Lame interneurale-L'épiderme de la lame constitue sur' les deux faces une assise de cellules dont les membranes sont épaissies. Ces cellules sont aplaties perpendiculairement à la surface toliaire et présentent de face un contour sinueux. L'épiderme est recouvert d'une épaisse couche de cuticule. Les stomates n'existent qu'à la face inférieure; ils sont elliptiques et entourés d'une assise concentrique de cellules épidermiques.

Le mésophylle est hétérogène. A la face supérieure existent deux ou trois couches de parenchyme vert en palissade, dont les éléments cylindriques et pressés les uns contre les autres, sont deux fois plus longs que larges. Quelques cellules, renfermant des cristaux agglomérés d'oxalate de chaux, se trouvent placées au milieu des éléments de ce parenchyme.

Le parenchyme inférieur, dont la puissance est un peu supérieure à celle du tissu en palissade, est constituté par des cellules qui se présentent en coupe transversale, sous la forme d'un cercle ou d'une ellipse allongée parrallèlement à la surface foliaire, et laissent entre elles de petits méats.

II. Nerrure. - La nervure est biconvexe. Sa portion centrale est occupée par le système médullo-libéro-ligneux. Ce dernier est très souvent composé d'un are libéro-ligneux inférieur, concave supérieurement, et d'une bande fibro-vasculaire supérieure reliantles deux extrémités de l'arc. Entre cette bande et l'arc siège un amas de cellules cylindriques ou prismatiques a base $p$ ronale, dont les parois un peu épaissies sont ponctuées et lignifiées. Certaines feuilles ne possèdent dans leur nervure que l'arc libéro-ligneux inférieur.

Le liber est formé. à sa pẻriphérie, de fibres à membranes tantôt minces et cellulosiques, tantôt épaissies, ponctuées et lignifiées. Le prosen. chyme est en contact arec les cellules libériennes.

Le bois est composé de nombreuses bandes vasculaires.

Le système libéro-ligneux est enveloppé par du parenchyme dont les éléments assez considérables sont cylindriques ou prismatiques à base polygonale. Ils laissent entre eux des méats; quelques-uns contiennont des cristaux. C'est dans ce tissu et tout contre le liber que sont placés (l os c'naux sécréteurs, le plus souvent en nombre impair. Chaque canal ¿t une section transversale polygonale, et est entouré d'une ou deux couches concentriques de petites collules remplies d'oléo-résine.

Le parenchyme méatique est situé entre deux massifs collenchymateux, l'un inférieur, l'autre supérieur. Les cellules qui entrent dans la constitution de chacun ont li forme de prismes dont la base est polygonale. Les parois cellulaires sont épaissies surtout aux angles, nacrées et ponctuées. Quant à l'épiderme, il est formé d'une assise de cellules épitissies allongées, suivant la nervure et revètues superficiellement d'un cuticule considéruble. 
FAMITIE DES OMUELLIERES

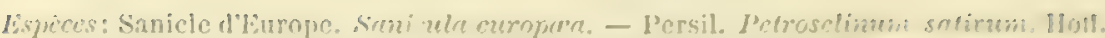

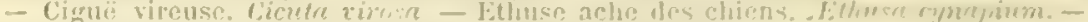

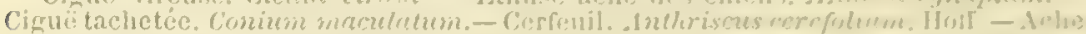

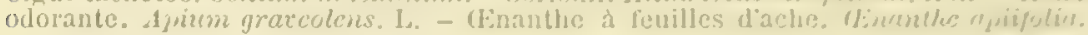

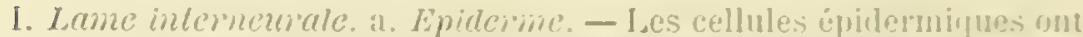
les parois sinueuses. Les stomates, disposés sans roldre et compris entre Irois, quatre ou cinq cellules, existent seulement dins lit julumint des cas

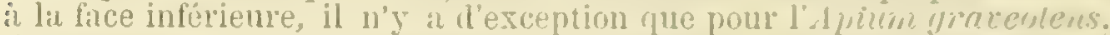
l'Enanthe apiifolia le Coninm qui en possident aux deus faces: l'épil. derme est dépourvu de glandes, on n'y rencontre d'ordinaire point de poils. Dans le cas ou ces derniers existent. ils sont unicellulaires.

b. Mésophylle. - Le mésophylle est hétérogrène asymétrirye, il ne présente ni glandes, ni cellules cristalligènes. Cependint le Sanicula renferme dans son parenchyme vert des ceilules qui contiennent des cristaux agglomérés d'oxalate de chaux.

II. Nervare. - L'épiderme de la nervure est composé de cellules allongées revètues à leur surfice de cuticule tantùt lisse (Anlhriscus cerefolium. Hoff̈. Ethusa cynapiume. L.), tantôt crèpée (Conium ancenlatum. L.), Cicuta virosa, Pelroselinum salivum. IIott. Sanicula curo. peer. L.). Contre l'épiderme est adossé un massif asse\% puissant de collenchyme. Dans la plupart des espèces cet hypoderme existe atur deux faces (pl. VI, fig. III, $h, b)$; mais dans la nervure des folioles du Conium maculatum. L. il n'y en qu'it la face inférieure. Entre les mas. sifs collenchymateux supérieur's et inférienr's sièrre un amas de yrandes cellules pàles (d) qui enveloppe un ou plusieurs cordons libéro-Iigneur. Chaque faisceau est composé à sa face inférieure de liber dont les éléments sont toujours cellulosiques; it la face supérieure de bois cons. titué par des vaisseaux trachéens, spiraux. Le bois est généralement limité en haut par une surface courbe à conrexité supérieure.

Le parenchyme entourant le système libéro-ligneux renferme des camaux secréteurs qui sont polyogonaux (coupe transrerse de la nerrure) et entourés de une à trois couches comprenant chacune cing cellules au plus. Dans Petrosetimun satirum l'Anthriscus cerefolium. le Conium machilatum, le Cicula virosa, l'Enanthe afuifulia, il n'existe qu'un canal ris-ti-ris de chaque faisceau libéro-ligneus, et toujours ce canal est situé à la face inférienre.

La Sanicula, l'Apium grareolens, l'Ethusa cynapium, possèdent au contraire des canaux au dos et au rentre de chaque cordon fibrovasculaile $(g, c)$.

Caractères communs. Point de glandes externes. Point de poils ou poils unicellulaires. Point de glandes internes. Point de cristaux doxalate (excepté en Sanicula europara L). - Collenchyme neural. - Citnaux sécréteurs dans le système fondimental.

Feuille de Sanicle d'Europe. - Sanicula europrea. I.

I. Lame intemenole. a. Epiderme. - L'épiderme, glabre et stomatique à la face inférieure seulement, est composé de cellules tabulaires à membrane mince, très sinueuses et recourertes d'une cuticule crètée. 
b. Mésophylle. - Le mésophylle est nettement hétérorène. Sous l'ẻpiderme supérieur sont deux couches de cellules allongées perpendiculairement ì la surface de la feuille. Les cellules de lassise superficielle sont eylindriques, et les plus grandes sont deux fois plus longues que larges. Les éléments de la couches profonde sont au contraire coniques et leur base s'appuie contre la première assise; leur longuenr ne dépasse jamais deux fois leur largeur. Ce parenchyme donne naissance à des cellules contenant des cristaux agrlomérés d'oxalate de chaux. A la face inférieure existent quatre ou cinq couches parallèles de cellules qui laissent entre elles de nombreux méats. Examinées de face, ces cellules offrent ordinairement quatre branches disposées en X. Sur une coupe transversaie, elles paraissent rectangulaires, à grand côté parallèle à l'épiderme.

II. Marge. - L'extrémitẻ latérale du mésophylle et de l'épiderme entre dans la constitution de la marge.

III. Nervure. - La nervure est proéminente sur les deux faces. Les cellules épidermiques sont allongées dans le sens longitudinai et présentent des parois épaissies, ponctuées, à surface crètée. Si l'on examine la nervure en allant de la partie inférieure à la partie supérieure, on trouve : $1^{0}$ de l'hypoderme inférieur; $2^{\circ}$ du parenchyme méatique inférieur; $3^{\circ}$ un cordon libéro-ligneux; $4^{\circ}$ du parenchyme méatique supérieur, et enfin de l'épiderme supérieur.

L'hypoderme est très développé aux deux faces. Ses cellules ont la forme d'un long prisme droit à base polygonale; elles sont blanches, très épaissies, et leurs membranes sont munies de ponctuations. Le parenchyme méatique constitue le massif dans lequel est plongé le système fibro-vasculaire. Situé entre les deux épidermes, il est formé de grandes cellules à membranes minces. Ces dernières sont polygonales, aussi bien dans le sens transтr'sal que dans le sens longitudinal, mais elles sont moins longues cependant que les cellules du collenchyme; les plus voisines de l'hypoderme inférieur contiennent des grains de cłlorophylle. C'est dans ce parenchyme qu'existent des canaux sécréteurs. A la face supérieure on en trouve un ou deux; quand il n'y en a qu'un, il est vis-à-vis le liber, sur la ligne médiane et á égale distance de ce dernier et du collenchy'me; lorsqu'il y en a deux, l'un, le plus considérable, a la position mème du canal unique; l'autre, plus petit, est latéral et touche le tissu libérien. Le canal, dans une section transversale, est polygonal, et est entouré par une couche concentrique de six à huit cellules. Quelques-uns des canaux sécréteur's existent à la face supérieure, et daus ce cas. ils sont à la limite du parenchyme méatique et d'un amas de cellules collenchymatoïdes existant à la face supérieure du bois.

Le liber a la forme d'un arc, il est composé de fibres minces et de cellules toujours cellulosiques. Le bois. plus développé que le liber, est formé de vaisseaux plongés dans un tissu collenchymateux qui s'étend mème au-dessus de lui.

$$
\text { Ache odorante. - Apium graveolens. L. }
$$

I. Lame interneurale. a. Epiderme. - L'épiderme, glabre, forme aux deux faces de la feuille une couche de cellules tabulaires dont le contour est sinueux. II produit des stomates.

b. Mésophylle. - A la face supérieure existe une assise de cellules cylindriques, perpendiculaires à la surface et de trois à cinq fois plus 
longues que latres. Au-dessons, se tronvent quelques conches parallites d'élements rameux, qui. sur une coupe transversale, sont allongés. paralledement it la surlice de lat feuille.

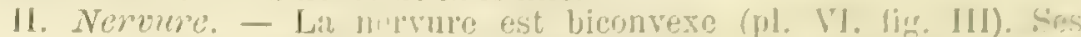
cellules épidermiques, allongíes suivant l'axe, sont revetues superfi.

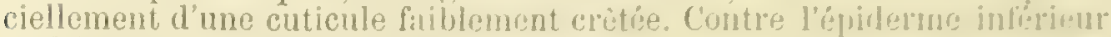
et lépiderme supérienr siogre un faiscenu de collenchyme (b.h). Les groupes collenchymateux limitent un amas cellulaire dont les elements assez grands laissent cntre eux de petits méats et ont la forme de polygones assez réruliers ( 1 ). Ce parenchyme entoure un cordon libíro. ligneux $(e, f)$ et donne nassance du des canaux sécréteurs (c, gr).

Le liber a la forme d'un arc concave supérieurement; il est conposé de fibres et de cellules dont les parois sont toujours cellulosiques.

Le bois convexe à si partie supérieure et inférieure est constitué par des vaisseaux placés irrégulierement dans du parenchyme dont les éléments présentent des membranes épaissies.

Il existe toujours un canal sécréteur au-dessus et au-dessous de chaque cordon $(c, g)$. Ces canaux ne touchent jamais le faiscenu fibrovasculaire; ils en sont séparés par quelques couches cellulaires. Certaines folioles possèdent en outre des canaux au roisinage des cordons. Chaque canal a une section transversale polygonale, et est entouré du une ou deux assises concentriques de cinq à sept cellules qui renferment de l'oléorésine.

Enanthe à feulles d'Ache. - Enanthe apiifolia. Brot.

I. Lume intemearale. a. Epiderme.-Une couche. Cellules ả contour sinueux. Stomates sur les deux faces.

b. Mésophylle. - Deux couches de parenchyme en palissade, à cellules supéricures quatre à cinq fois plus longues que larges. Parenchyme inférieur égal ou inférieur en puissance au supérieur.

II. Nerure. - Convexe inférieurement, concave superieurement. Epiderme recouvert de cuticule crètée. Collenchyme aux deux fuces. Ln cordon libéro-ligneux. Quelquefois deux, l'un inférieur, l'autre supérieur. Il n'y a jamais qu'un canal sécréteur vis-i-vis chaque cordon.

Feuille de Persil cultive. - Petroselinum satirum. Hofim.

I. Lame interneurale. a. Epiderme. - L'épiderme, glabre, et pourru destomates à la face inférieure seulement, est composé sur les deux faces d'une seule assise de cellules tabulaires, sinueuses superficiellement; ces éléments ont leur membrane mince et sont recourerts d'une mince couche de cuticules présentant de faibles crètes qui donnent ả la membrane supérieure un aspect strié.

b. Mésophylle. - Le mésophylle pourvu de chlorophylle ne contient pas de cellules cristalligènes. A la face supérieure, le mésophylle constitue une couche de cellules cylndriques disposées en palissade et dont la longueur ne dépasse pas deux fois la largeur. A la face inférieure, le mésophylle est méatique; il forme en général quatre couches parallèles dont l'ensemble est plus développé que l'assise en palissade. Les celIules, rectangulaires, lorsqu on les esamine en couje tranversale, sont 
rameuses a trois ou quatre branches courtes, lorsqu'on les regarde de face.

II. Nervure. - La nervure, biconvexe, est limitée aux deux faces par de l'épiderme glabre et dépourvu de stomates; elle est composé d'une assise de cellules très allongées parallèlement à la nervure, présentant à la superficie des stries parallèles entre elles et aux grand côté des cellules. En dedans des deux épidermes, se remarque un massif assez puissant de cellules collenchymateuses; entre les collenchymes inférieur et supérieur siège le tissu cellulaire dont les éléments à parois minces et à contour polygonal sont juxtaposés, allongés parallèlement ì l'axe de la nervure et séparés les uns des autres par des parois ordinaire. ment horizontales. C'est dans ce massif que l'on remarque un cordon libéro ligneux et un canaI. Ce dernier, assez considérable, existe à la face inférieure et se trouve placé vis-à-vis du cordon libéro-ligneux et contre le liber. Sa sertion transversale est jolygonale et il est entouré d'une couche concentrique de cinq à huit cellules sécrétrices.

Feuille de Ciguë vireuse. - Cicuta virosa. L.

I. Lame interncurale. - L'épiderme, glabre, dépourvu de stomates à la face supérieure, est formé d'une seule couche de cellules tabulaires. A la face inférieure, ces éléments ont leur membrane sinueuse, tandis qu'à la supérieure, les sinuosités sont très faibles. Les cellules épider"miques sont recouvertes d'une cuticule crêtée.

Le mésophylle est hétérogène. Au-dessus de l'épiderme supérieur existe une couche de cellules cylindriques disposées en palissades de trois à quatre fois plus longues que larges. Les cellules de la face inférieure sont rameuses. De face, elles ont un contour très sinueux sans avoir la forme d'un X. En coupe transversale, elles sont pour la plupart allongées parallèlement à l'épiderme. Ces cellules forment quatre ou cinq assises parallèles dans lesquelles n'existe aucune cellule cristalligène.

II. Nervure - La nervure est proéminente sur les deux faces. Sonépiderme présente de fortes saillies coniques et la cuticule offre des crêtes nombreuses qui donnent à la membrane épidermique un aspect strié, lorsqu'on la regarde de face. Contre les deux épidermes s'appuie un faisceau de collenchyme auquel est adossé une masse parenchymateuse. Cette dernière enveloppe un cordon libéro-ligneux et renferme ia la face inférieure un canal sécrétev:. Celui-ci, situé vis-à-vis le liber, est bordé de six à sept cellules sécrétrices.

Feuille de Ciguë taclietée. - Conium maculatum.

I. Lame interneurale. a. Epiderme.-L'épiderme, glabre, et pourvu de stomates tantôt sur la face inférieure seulement, tantôt sur les deux faces, est constitué par une assise de cellules tabulaires. A la face infé. rieure, les parois sont très sinueuses, tandis qu'ả la face supérieure elles sont friblement ondulées. Elles sont revêtues superficiellement par une couche mince de cuticule, présentant de faibles crêtes qui donnent un aspect finement strié à l'épiderme lor'squ'on examine ce dernier de face.

b. Mésophylle. - Le mésophylle, toujour's chlorophyllien, est dépourvu de cellules cristalligènes. Au-dessous de l'épiderme supérieur 
existe me conche de cellules cylindrigues de trois is quatre fois plus longues que larges et disposés en palissale. A la ficce inforienres se trouve une lame composie do plusieurs assises parallobes do relludes rameuses. Ces dernieres sont le plus sonvent allonges patralliglemmat it la surface de l'épiderme. Vues de face, elles ollirent plusiens prolonerements an nombre de trois ou quatre, et qui se joignemt entre cux en laissant des méats polyononatux considélables.

II. Marye. - Isa marge est uniquement constituce jar du mésoplylle recouvert d'epiderme.

III. Nervurc. - La nervure est seulement proininente il lat filce inférieure. La face supérieure est excavéc. Son épillerne crêtée présente parfois des saillies coniques. Le collenchyme niexist- rue sur la fitce inférieure, et cncore n'est-il que très peu développé puisquil fonrnit au plus trois couches.

Quant au parenchyme et au cordon libéro-limeux. il a la méme conposition que dans les autres ombelliféres. Il n'y a jamais qu'un canal secréteur au dos de chaque arc libérien, et le canal est placé tout contre l'are.

\section{Feuille d'Ethuse ache des chicns. - Ethusa cynayium L.}

I. Lame interneurale. a. Epiderme. - La lame est recouverte d'un epiderme dont les cellules tabulaires sont sinueuses il leur surface of ont des membranes extrêmement minces. L'épiderme inférleur est seul pour'ru de stomates.

b. Mésophylle. - Le mésophylle, variable suivant la face, est lépourvu de cellules cristalligenes. A la face supérieure, il forme une couche de cellules cylindriques pressées les unes contre les autres, allongées perpendiculairement ia la surface et en général trois fois plus longues que larges. A la face inférieurce, se trouve le parenchrme vert ì méats, aussi développé que le supérieur; il forme trois ou quatre couches parallèles de cellules qui, en coupe transversale, paraissent la plupart rectangulaires, allongées parallélement it la surface. Vus de face, ces éléments sont rameux, ordinairement à trois branches et laissent entre eux des méats irréguliers.

II. Marge. - La marge est uniquement constituée par la terminaison latérale du mésophylle recouvert d'épiderme.

III. Nervure. - La nervure est biconvexe; elle est limitée ì ses deus faces par de l'épiderme dont les éléments ont un contour rectangulaire et sont allongées parallèlement au grand axe de la nerrure.

L'épiderme, dépourvu de stomates sur les deux faces, est parfois pourvu à la face supérieure de gros poils coniques unicellulaires et à membrane un peu tuberculeuse. Coutre l'épiderme supérieur existe un petit massif de cellules pailes ì membrane épaissie et à contour polyconal. Ce massif constitue un collenchyme. En dedans de l'épiderme inférieur se trouve parfois une couche d'éléments semblables. Entre ces deux collenchymes siège une masse de cellules au milieu de laquelle est placé un cordon libéro-ligneux semblable au cordon des ombellifëres en général. Les cellules du parenchyme, assez grandes, ont un contour polygonal et sont allongées parallèlement au grand axe de la nerture. Dans ce tissu se trourent des canaux sécréteurs tant à la face inférieure qu'à 
la face supérieure. Ces canaux polygonaux, sur une coupe transtersale, sont entourés d'une couche concentrique de cellules sécrétrices plus ou moins nombreuses. Il existe un canal à la face supérieure et un autre plus grand à la face inférieure, vis-it-vis de chaque cordon fibro-vasculaire.

$$
\text { Feuille de Cerfeuil. - Anthriscus cerefolium. Hotfm. }
$$

I. Lame intemcurale. a. Epiderme.-La lame est limitée à ses deux faces par une assise de cellules épidermiques présentant une membrane mince, sinueuse et lisse. L'épiderme inférieur est seul pourvu de stomates.

b. Mésophylle. - Le mésophylle vert est hétérogène. A la face supéricure, il forme une couche de cellules presque cubiques ou cylindriques disposées en palissade dont la longueur' n'atteint jamais deux fois la largeur. A la face inférieure sont des cellules rameuses formant d'ordinaire trois ou quatre couches parallèles qui, dans leur ensemble, ont une puissance plus grande que celles de la couche supérieure. De face, ces éléments sont rameux à trois ou quatre branches, tandis qu'en coupe transtersale ils paraissent rectangulaires ou elliptiques à grand diamètre parallèle à la surface foliaire.

II. Norvure. - Convexe inférieurement et excavée supérieurement, la nervure a l'épiderme dépourvu de stomates; les éléments sont allongés parallèlement à la nervure. L'ẻpiderme donne naissance ì des poils coniques unicellulaires de longueur variable, dont la membrane est épaissie et un peu tuberculeuse. En dedans de l'épiderme se trouve une masse de cellules à parois minces dans laquelle sière un cordon libéroligneux. Vis-ì-vis de ce dernier et contre le liber existe un canal sécréteur assez développé.

FAMILLE DES VACCINIÉES.

Feuille d'Airelle rouge. - Vaccinium ritis idoa. L.

I. Lame interneurale. a. Epideme. - Les deux épidermes sont formés d'une couche de cellules tabulaires à contour sinueux, et dont les parois épaissies sont revêtues à leur surface d'une cuticule considérable. L'épiderme inférieur seul possède des stomates et donne nais. sance à des glandes. Ces dernières ont la forme de massues; elles sont composés de plusieurs ranqées cellulaires, et sont supportées par un pédicelle cylindrique constızué par deux ou quatre files verticales de cellules dont la longueur ne dépasse pas deux fois la largeur.

II. Mésophylle. - Si l'on fait une coupe transversale d"une feuille, on remarque entre les deux épidermes un mésophylle dénourvu de cellules cristalligènes, et dont les éléments différant sur les deux faces renferment dans leur intérieur de la chlorophylle et du tannin. Au-dessus de l'épiderme supérieur se trouvent trois ou quatre assises de cellules cylin. driques, perpendiculaires à la surface de la feuille, et de deux à quatre fois plus larges. Ce tissu en palissade est en contact par sa partie inférieure avec des cellules circulaires, elliptiques ou rameuses dirigées en tous sens et laissant entre elles de grands espaces.

III. Marge. - Contre l'épiderme marginal s'appuie un faisceau de fibres dont la section transversale est polygonale. Celles-ci ont une paroi très épaisse, lignifiée, et une cavité très faible (pl. V, fig. V, a). 
IV. Nerzure. - Les cellules epidermirues, fosminces de faco. sont polygonales, non allongées suivant laxo de la mervure. J épliderme

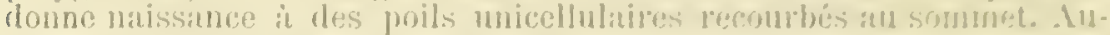

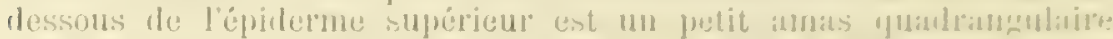
composé de unc atrois couches de petiles cellules dont los pratris somt

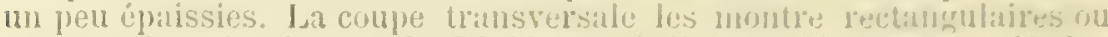
polyonales. Au-dessus de l'épiderne infépreur siéry un inassif plus considerable et formé de cellules dont les rmembranos épaissies sone ponctuées. Ces cellules paraissent ovales sur une coupe perpendiculainc it l'axe de la nervure. Intre le parenchyme supérieur et le yatenchrine

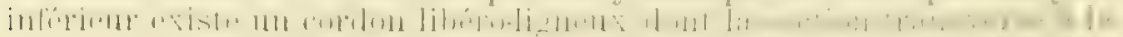
forme d'un triangle i base inféricure et il sommet supérient. Il est conposé en allant de lia base vers le sommet: $1^{\circ}$ d'un are de libres libé-

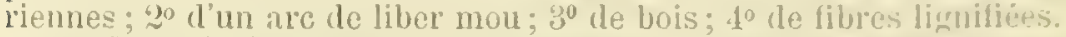

I es fibres libéxiennes forment plusieurs couches disposies en un arc continu. Leur memblane est très épaissic et lignifice, tandis que leur cavité est peu consiclérable. Le bois, plus développé que le liber, est composé de fibres, de vaisseaux (ponctués, spiraux) et de trachées. (buant aux fiores situées au-dessus du bois, elles ont la mème constitution que celles qui existent a la face inférieure du faiscenu.

Cette espỏce peut ètre substituée à l’Ura u’si ou citre confondue avec la feuille de Myrtus communis. L.

\section{FAMILLE DES ÉRICINEES.}

Especes: Arbousier unedo. Arbulus unedo. L. - Uva ursi. Arctostajhylas uta ursi. Spreng. -Gaultherie couchéc.Gaultheria procumbers. L.-Anilromede is feuilles de polium. Antromeda polifolia. L. - Ledon des marais. Ledum pnlustre. L.

I. Lame interneurale. a. Epiderme.-l'loutes les Ericinées possẻdent des cellures épidermiques dont les membranes sont épaissies et revètues d'une couche assez considérable de cuticule. Le contour de ces cellules. polygonal dans l'Uva ursi et l'Arbutus unedo. L., est légèrement sinueux dans le Gautheria procumbens. L. Il n'existe des stomates qu'à la face inférieure.

La plupart des espèces, à l'exception du Ledum, sont dépourrues de poils et de glandes.

b. Mésophylle. - Le mésophylle hétérogène asymétrique renferme le plus souvent des cellules cristalligènes. Il forme toujours it la face supérieure deux ou trois couches de cellules cylindriques, allongées perpendiculairement ¿̀ l'épiderme. Le parenchyme infélieur est méatique. Dans l'Andromeda, toutes les cellules du mésophylle sont allongées perpendiculairement à la surface de l'épiderme.

II. Marge. - 'Tantôt la terminaison du parenchyme rert constitue la marge (Arbutus unedo. L., Andromeda poliifo?ia. L., Leduin. L.), tantôt il existe contre l'épiderme marginal un faisceau de cellules épaissies incolores et it membrane cellulosique ( $U \tau^{\circ} \alpha$ usi) ou de fibres lignifiées (Gaultheria procumbens).

III. Nervure. - Dans l'Ura usi et l'Arbutus unedo il y a contre fes deux épidermes un tissu cellulaire homogène dont les éléments ont es parois très épaisses et ponctuées, tissu dans lequel est placé le sjs. ème líbéro-ligneux. 
Dans le Ledum, l'Andromeda et le Gaultheria, le parenchyme diffère suivant la face. A la face supérieure et tout contre l'épiderme se trouve un petit massif a section transversale quadrangulaire, composé de cellules dont les parois présentent un épaississement assez considérable. A la face inférieure existe un tissu hétérogène, qui, dans l'Andromeda et le Gaultheria est formé de petites cellules à membrane épaissie, ponctuée, formant entre elle des réseaux dont les mailles sont occupées par des cellules grandes et minces. Que le parenchyme soit homogène ou hétérogène, il renferme des cellules à cristaux agglomérés (Gaultheria procumbens) ou isolés (Aruutus unedo. Uta ursi).

Le système fibro-vasculaire est représenté par un seul are dans l'Ura ursi, le Gaultheria, l'Andromeda, ou par deux arcs, l'un inférieur, l'autre supérieux dans l'Arbutus unedo. Les fibres libériennes sont tantôt lignifiẻes (Gaultheria, Andiomeda), tantôt cellulosiques (Uva rersi).

Quant au bois, il est formé de bandes rayonnantes très serrées de vaisseaux et de fibres.

Caractères communs: L'épiderme inférieur seul pourvu de stomates. Tissu en palissade ì plusieurs couches. 'Tissu situé entre l'épiderme supérieur et le faisceau, homogène ou hétérogène mêlé (nous n'avons rencontré ce dernier tissu que dans les Ericinées). Point de glandes internes. Des cristaux d'oxalate de chaux.

Arbousier unedo. - Arbutus unedo. L.

I. Lame interneurale, a. Epideme. - L'épiderme, glabre, ne possédant des stomates qu'i la face inférieure, est constitué par une seule assise de cellules a membranes un peu épaissies, recouvertes d'une couche épaisse de cuticule. Elles sont tabulaires et irrégulièrement polygonales à la superficie. Les stomates ellliptiques sont bordés de cellules polygonales rayonnantes.

b. Mésophylle. - A la face supérieure, le mésophylle forme deux ou trois conches de cellules cylindriques de six ì sept fois plus longues que larges et disposées en palissade. Ces cellules renferment de la chlorophylle, des gouttelettes huileuses et du tannin.

A la face inférieure, les cellules ne sont pas rameuses, mais sphériques, cylindriques ou ovoïdes; elles ne laissent entre elles que de faibles méats: Les inférieures, voisines de l'epiderme, sont presque toutes allongées perpendiculairement à la surface foliaire.

II. Marge. - La marge est constiluée par du parenchyme vert recouvert d'épiderme.

III. Nervure. - La nelvure, biconvexe, est limitée par une assise épidermique ì cellules tabulaires ordinairement rectangulaires superficiellement. Contre l'épiderme siége un amas de cellules à membranes épaissies, réfringentes, cellulosiques. Ces éléments ont la forme de prismes it base polygonale, de deux it trois fois plus hauts que larges. Quelques-uns renferment des cristaux isolés d'oxalate de chaux.

Entre ces deux massil's cellulaires siege un cordon dont la section transversale a la forme d'une ellipse it grand diamètre transverse. Il est composé de deux arcs libéro-ligneux tomnant leur bois l'un vers l'autre et séparés par' des cellules médullaires.

Chaque arc est constitue par du liber et du bois. 
Le liber est fibreux il sa smface; mais ses fibres lignifien fet ipals.

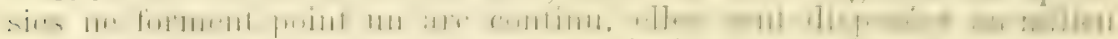

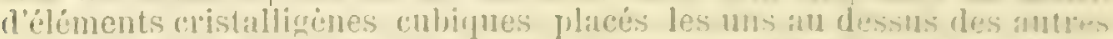

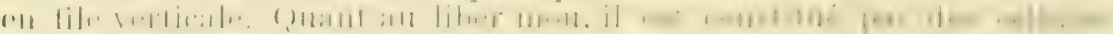
dont la plupart sont remplies de cristaux simples d'oxalate de chatux.

Le bois, un peu plus développé ane le liber, est composio dle bandes

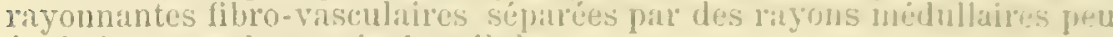
çnas it une seule rangrie de cellules.

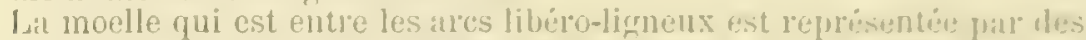
cellules it membrane épaissie. limnitiéc et ponetuép. Ces cellutes ne latissent entre elles ancun meat ct elles sont carrées ou rectangulairos en coupe longitudinale, anrondies on ovales dins le sens transwersal. Quelques-unes contiennent des cristanx.

Arctostaphylos busserole. - Arclosiaphylos urr-ursi. Spreng.

1. Lame intèneuiale a. Elviderme. L Lépiderme supérieur. xeconvert d'une très épaisse conche cuticulaire. dépassant la moitié de la cavité cellulaire et sútendant sur les fices latérales de ces dernieres cellules, est composé d'une seule couche d'éléments qui. vus de face. sont polygonaux, et en coupe transversale sont rectangulaires. Cet eppiderme est dépourvi de stomates.

L'épiderme inférieur a des cellules semblables, un peu plus petites

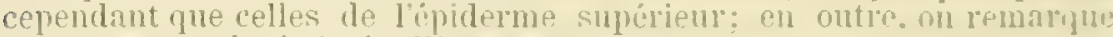
des stomates placés irrignlierement.

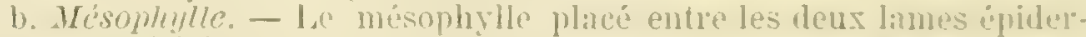
miques est hétérogene. Les úluments qui le constituent ronferment de lir chlorophylle. Les cellules retes qui touchent it lépiderme supéricur sont eylindriques, de deux i quatre fois plus lnnerues que larges et perpendiculaires ì lia lame foliaire. Elles forment une lame de trois á cinq conches opposécs entre elles, d'une épaisscur éritle à la lame formée pur le parenchyme vert infériem.

Le mésophylle infëriem est constitué par des collules épaissies yui se présentent sous des formes diverses dans une coupe transversale : les unes sont rectanguldires, dautres, tres irrérulierement polyonales. d'autres, arondies. Filles laissent entre elles des lacunes assez considérables.

II. Marge. - A la marre, existent, contre lépiderme, deux ou trois couches de cellules prismatiques, ir membrane cellulosique. épaisse. ponctuée et enveloppant une carité assez large.

III. Norure. - Los deux épidermes présentent les mèmes particularités que ceux de la lame foliaire: mais ils sont dépourvus de stomates et leurs cellules sont allonrées suirant la nerrure.

Entre ces épidermes se trouvent du tissu hypodermique au centre duquel se toit un arc rasculaire (pl. VI, fig. IV). L'hypocierme supéricur forme un amas presque quadrangulaire sous une coupe transrersale. II est composé de cinq it sept couches de cellules a membranes épaisses, réfingentes, polygonales (de $t$ a 6 angles) en coupe transrersile: rectanrulatires et allonrées dans une coupe longitudinale. Ces cellales, qui présentent de très petites ponctuations arrondies, possèdent. les unes des grains damidon, les autres de gros cristaux isolés doralate de chaux. La couche inférieure de cet hypoderme touche le système 
fibro-vasculaire. L'hypoderme inférieur, le plus étendu tangentiellement, a les éléments un peu plus gros; de plus, il ne renferme que très peu d'amidon, parfois même il n'en renferme point. La couche supérieure est en contact arec le liber.

Systeme fibro-zasculaire. - Le faiscean a la forme d'une ellipse à grand diamètre latéral. Le liber inférieur (c) ne renferme pas de fibres, mais on y trouve des éléments séveux et cristalligènes (oxalate) à membranes assez épaisses. Son épaisseur est moindre que celle du bois.

Le bois est constitué inférieurement par un massif puissant de fibres ponctuées disposées radialement, et supérieurement par quelques raisseaux spiraux, spiro-annelés et trachées. Les files radiales de ces vaisseaux s'avancent un peu supérieurement.

Entre plusieurs files radiales de fibres et vaisseaux, on remarque des rayons médullaires constitués par des cellules à membrane mince contenant des granulations brunes.

Suhstitutions.

$1^{\circ}$ Feuilles de Buis (Buxus sempervirens. L.).

C'est surtout par' la constitution de la nervure que le Buis se distingue de l'Ura ursi. Le parenchyme neural stipérieur est constitué par des cellules ì chlorophylle allongées perpendiculairement ì la surface de la feuille (pl. VI, fig. I, h). Ces cellules, ì membrane assez mince, se continuent it droite et à gauche arec les éléments en palissade de la lame qui ont la mème forme qu'elles. Le faisceau libéro-ligneux elliptique à grand diamètre latéral présente ì sa face inférieure et ì sa face supérieure des fibres épaissies, lignitiées.

$2^{\circ}$ Feuilles d'Airelle rouge (Traccinim ritis idea. L.).

L'épiderme inférieur de l'Airelle olrie des glandes en massue ou ellipsoüdales composées de plusieurs cellules séparées entre elles par des cloisons qui sont dirigées dans tous les sens. Le pédicelle qui le soutient est plurisérié 'Toute la feuille est clépourvue de cellules cristalligènes. Le bord foliaire présente un cordon de fibres lignifiées (pl. V, fig. $Y$, a), et il existe à la face supérieure ainsi qu'ả la face inférieure des faisceaux libéro-ligneux, des fibres épaissies, lignifiées.

\section{Gaulthérie couchée. Gautheria procumbens. L.}

I. Lame interneurale. a. Epiderme. - L'épiderme forme une seule assise de cellules à membranes épaissies. ponctuées, revêtues d'une couche épaisse de cuticule qui empiète sur les parois latérales. Ces cellules sont rectangulaires en coupe transversale, légèrement sinueuses quand on les roit de face.

L'épiderme inférieur est seul pourvu de stomates; ces derniers, très nombreux sont elliptiques. Contre le boril externe de la plupart des stomates sapplique une cellule en croissant dont la paroi interne con. cave legarde ces orifices et dont la paroi externe est anguleuse, légèrement sinueuse.

b. Mésophylle. - A la fice supérieure, le mésophylle forme trois ou quatre couches de grosses cellules cylindriques ordinairement deux fois plus longues que larges et disposées en palissade. La plupart de ces cellules contiennent de la chlorophylle et des gouttelettes d'huile jau- 
natre : quolques-unes, incolores, renforment des cristanx agrghomerés d'oxirlate de chintx.

An dessus de ce prarenchyne vient un parenchyne forme de cellules rancuses laissant entre elles des lacunes, collules qui. fon conpe transversale, sont arrondios, angulenses on rectingulaires diriguescu trus sens. et qui, vues de face, sont rameuses it trois on ruatre bunches tris contes. Quelques-unes conticnnent des cristaux.

II. Marge - Contre l'épiderne mareninal s'apjuie un faiscean ule fibres tres épaisses et lignilies, polygonales sun tue coupe trinsten" sile.

111. Terzure. - La nervure, convexe inféricuromont. est un pen excavée ì la surface supérieure. Son épiderme al des rellules épisisies. restangulaires et dont le grand côté longiturlinal est an jolus deux fuis plus grand que le cote transversal.

Au dessus de l'épiderme inférieur se tronve en général une couche de cellules dont les parois sont épaissies. ponctuées. Ces éléments. rui ne laissent entre eux ancun méat, sont elliptiques a grand diamèce tangentiel; et dans le sens longitudinal, ils sont rectangulaires.

En dedans de cette assise existe un massif de cellules un peu colorees en vert et laissant entre elles de grands méats. Les unes, in parois épaisses et ponctuées, sont d'ordinaire ovales ou elliptiques: les antres: plus grandes, ont leur membrane mince. Quelques-unes renferment des cristaux agrolomérés d'oxalate de chaux.

Contre lépiderme supéricur et sur la ligne médiane sière un massif cellulaire en général quadrangulaire. Les éléments qui le constituent ont les parois très épaisses, ponctuées et cellulosiques. Un petit nombre produisent des cristaux daus leur cavité. Sur une coupe transrersale. ils sont rectangulaires ou elliptiques. trés serrés les uns contre les autres; sur une coupe longitudinale. ils ont la forme d'un rectangle allongé. Les pans latéraux de ce massif hypodermique sont en contace avec le parenchyme vert supérieur de la lame.

C'est entre cet hypoderme et te tissu méatique inférieur que se trouve un faisceau à section transverse elliptique. Il est composé en allant de la partie supérieure à la partie inférieure de la nervure :

$1^{\circ}$ De liber fibreux à si prartie inférieure. celluleux à sa portion supérieure Les fibres dont lat section transversale est polygonale forment un arc continu; elles ont les membranes lignifiées, trés épaissies, de manière à ne laisser qu'une faible cavité intérieure:

$2^{\circ}$ Le bois, plus développé que le liber, est composé de bandes rayonnantes fibro-vasculaires, séparées par une rangée de cellules allongées parallèlement aux lames ligneuses;

$3^{n}$ De quelques cellules à membrane un peu épaissie, lignitiée et ponctuée, s’appuyant inférieurement contre le bois:

4" D'un massif de fibres assez larges ou fibres libériennes inférieures.

Andromède à feuilles de polium. - Andromeda poliifolia. L.

I. Lame interneuralc. a. Epiderne. - L'épiderme, glabre, et muni de stomates ì la face inférieure seulement, est composé d'une assise de cellules tabulaires dont le contour est polygonal ou légèrement sinueux. Les parois de ces cellules, assez épaisses, sont recouvertes ì la surface d'une couche considérable de cuticule. 
b. Mésophille. - Les cellules vertes qui sont situées entre les deux épidermes sont tontes dirigées perpendiculairement à la surface de la feuille. Les éléments supérieur's cylindriques sont pressés les uns contre les autres, tandis que les inférieurs, ellipsoïdaux ou un peu rameux, laissent entre eux des espaces assez considérables. On ne rencontre dans ce parenchyme aucune cellules cristalligènes.

II. Marge - La terminaison latérale du mésophylle, revêtue d'ẻpiderme. constitue le bord foliaire.

III. Nervure. - Les cellules épidermiques sont allongées suivant l'axe de la nervure. Leur membrane est très épaisse et se trouve recouverte d'une cuticule d'une grande épaisseur. Au-dessus de l'épiderme inférieur siège du parenchyme composé de deux sortes d'éléments entremèlés : les uns, petits, arondis, ont une membrane épaisse; les autres, très grands. sont polygonaux et possèdent des parois minces. Les premiers de ces éléments renferment, un certain nombre du moins, des cristaux d'oxalate de chaux. Ce tissu est en contact supérieurement avec un cordon libéro-ligneux dont la section transtersale a la forme d'un triangle à base courbe inférieure et à sommet supérieur. Le cordon est formé en allant du bas ver's le haut:

10 De plusieurs couches de fibres libériennes, dont les enveloppes lignifiées sont très épaisses, et dont les cavités sont extrêmement faibles;

20 De liber mou celluleux moins développé que le prosenchyme ;

$3^{\circ}$ De bois composé de fibres ponctuées, de vaisseaux ponctués, spiraux et de trachées:

$4^{\circ}$ D'un amas triangulaire de fibres semblables à celles qui existent à la face inférieure du faisceau. Entre cette masse et l'épiderme supérieur siège un petit groupe cellulaire dont la coupe transversale est quadrangulaire, et dont les éléments sont arrondis ou polygonaus (coupe transversale), à parois assez épaisses.

Cette espèce peut être confondue avec le Rosmarinus officinalis. L.

Ledon des marais. - Ledum palustre. L.

I. Lame interneurale a. Epiderme. - L'épiderme est composé d'une couche de cellules tabulaires dont le contour est sinueux. Recouvert superficiellement de cuticule épaisse, il est muni à la face inférieure de stomates, de poils ordinaires et de poils glanduleux. Les poils ordinaires sont de plusieurs sortes. Les uns, courts, sont unicellulaires et coniques; d'autres sont formés d'une rangée de cellules d'autint plus longues qu'elles sont voisines du sommet; d'autres enfin sont constitués par deux séries parallèles de cellules allongées. Les poils des deux dernières sortes sont très longs et leurs parois sont garnies de tubercules.

Parmi les glandes que l'on rencontre sur l'épiderme inférieur, les unes, volumineuses, sont formés d'une rancée horizontale de plusieurs cellules; les autres, plus petites, sont unicellulaires. Toutes sont arrondies et portées par un pédicelle unisérié dont les éléments sont toujours plus larges que hauts. Quelquefois le pédicelle est constitué par deux files verticales de cellules. Les glandes renferment dans leur intérieur des substances oléo-résineuses de couleur brunitre.

b. Mésopluylle. - Au-dessous de l'épiderme supérieur se trouvent trois ou quatre cellules cylindriques perpendiculaires à la surface de la 
feuille, ot de deux i cing fois plus lonerues que laneres. Cos callules con. liennent de lat chloropliylle, senlement primi alles se rencontrent dows

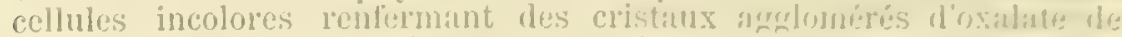
chaux. Ces dernières existent surtout dans les conches mofordues du tissu en palissade. Au-dessous de crlui-ri est une lune de putitra cellules vertes mêlées à de rrandes cellules incolones.

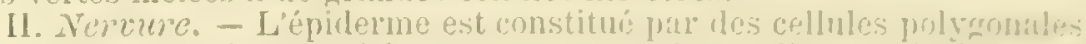

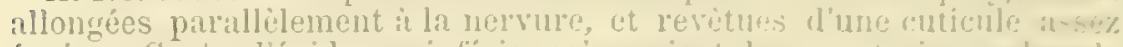

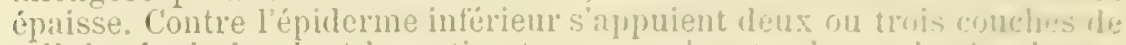
cellules épaissies dout la section transiel'sale est polyoronale. An-drasus de ces dernières existent deux ou trois assises de mrindes cellules inco. lores dont les parois sont minces. Quelques-unes d'entre chles renferment des cristaux agromérés. Ce parenchyme est en contuct supélieurement avec des cellules plus petites dont les nembranes sont filus épaisses. Les unes contienuent de l'oxalate de chaux, d'autres des matières oléo-résineuses.

Un cordon libéro-ligreux, dont la section transrerse est demi-circulaire, touche la partie supérieure de ce massif cellularre. Le filiscean est composé en allant du bas vers le haut: $1^{\circ}$ de liber; 20 de bois; $3^{\circ}$ l'un massif de fibres lignifiées: $4^{\circ}$ de deux ilots libéliens.

Le liber est forné de fibres et de cellules. Les fibres. cellulosinues dans certaines feuilles, se lignifient; dans d'autres elles constituent un arc continu. Le bois a la forme d'un arc concave supérieurement. Il est composé de fibres, de raisseanx ponctués, spiraux et de trachées. Les fibres situées au-dessus du bois sont lignifiées et plus petites que les fibres libériennes. Enfin les Îlots du liber supérieur ont la mème constitution que le liber inférieur. C'ertaines feuilles ne possédent pas de liber supéricur. Entre le cordon fibro-rasculaire et l'épilerme supéticur siège un massif cellulaire dont la section transverse est rechangulaire. Les éléments qui le constituent ont la forme de cylindre dont lit lon:ruen: ne dépasse pas deux fois la largeur. Leurs parois sont atssez éplisses. La plupart contiennent des cristaux

Les feuilles de cette plante pourraient itre prises pour celles de liusmarimus officinalis. L.

\section{FAMILLE DES PYROLACÉES.}

Tímaphile en ombelle. - Chimaphila umbellata. sutt.

I. Lame interneuralc. a. Epiderme. - L'épiderme. slibre. et pourru de stomates à la face inféricure seulement. est composé d'une seule assise de cellules tabulaires, simueuses superticiellement. Ces éléments. à membrane très épaisse, ponctuée, sont revitus d'une collche trés épaisse de cuticule qui sétend un peu sur les parois latérales. Les stomates, presque arrondis et disposés sans ordre, se trourent placés entre trois ou quatre cellules de l'épiderme.

b. Mésophylle. - Le mésophylle, dont presque tous les éléments renferment de la chlorophylle et du tamin, présente it la face supérieure deux assises de cellules cylindrigues en geneiral trois lois plus longues que larges et disposées en palissade. Al la fice inférieure, au contritie. existent des cellules rameuses, laissant entre elles de grands méats. Si 
on examine une coupe transversale, elles paraissent arrondies, ovales ou elliptiques et disposées en tous sens. De face, elles sont rameuses, d'ordinaire ì deux ou trois branches très courtes. Ce parcnchyme inférieur, un peu plus développé que le supérieur, possède de grandes cellules renfermant des cristaux agrglomérés d'oxalate de chaux.

II. Marge. - Des cellules vertes recouvertes d'épiderme constituent la marge.

III. Nervure. - En dedans d'un épiderme à cellules épaissies, ponctuées et rectangulaires superficiellement, se trouvent de grandes cellules à membranes épaissies, nacrées et ponctuées. Plus nombreuses ì la face inférieure qư la face supérieure où elles forment seulement deux couches, ces cellules sont carrées ou rectangulaires sur une coupe transrersale; arrondies, elliptiques ou fuiblement polygonales sur une coupe longitudinale. Leur cavité, très grande, contient des grains de chlorophylle et du tannin, ou des cristaux agglomérés d'oxalate de chaux.

Les cellules de la face infẻrieure sont d'autant plus larges, plus épais. sies et moins longues qu'elles sont plus rapprochées de l'épiderme.

Entre la couche cellulaire inférieure et la couche cellulaire supérieure siège un arc libéro-ligneux convexe inférieurement, concave supérieurement, et dans la carité duquel se trouve un petit amas cellulaire formant une espèce de moelle. Le liber, peu développé, est formé d'ẻléments dont les parois sont cellulosiques.

Le bois est constitué par des lames de fibres et vaisseaux, lames séparées par des rayons médullaires minces ì une rangée de cellules épaissies.

Quant aux éléments situés dans la concavité supérieure du bois, ils ont la forme de longs prismes droits it base polygonale. Leurs parois sont épaisses, surtout aux angles, et sont de nature cellulosique. Ils renferment presque tous du tannim.

FAMILLE DES OLÉINÉES.

Feuille de Frène élevé. - Fraximus excelsior. L.

I. Lame internerrale, a. Epiderne. - L'épiderme inférieur est composé d'un amas de cellules tabulaires à contour sinueux. Ces cellules sont recouvertes d'une couche de cuticule qui présente des crêtes ì sa surface; les stomates, elliptiques, sont entourés de plusieurs utricules épidermiques.

L'épiderme supériem est dépourvu de crètes et de stomates, il donne naissance à quelques glandes à pédicelle unicellulaire extrèmement court enfoncé dans une dépression de l'épiderme. Ces glandes, très aplaties, sont composées d'une seule rangée de cellules séparées les unes des autres par des cloisons verticales.

b. Mésophylle. - Le mésophylle vert, dépourvu de cellules cristalligènes, est formé, a la face supérieure, de deux couches de cellules cylindriques disposées en palissade et dont les superficielles sont en général de quatre ì six fois plus longues que larges. A la face inférieure existent des cellules rameuses constituant par leur ensemble une lame égale ì celle du parenchyme supérieur. Sur une coupe transversale, les cellules sont rameuses, dirigées en tous sens et laissent entre elles des méats plus ou moins considérables. 
II. Nervure - Ijépilerme de la nervure, diepourva de stomatra.

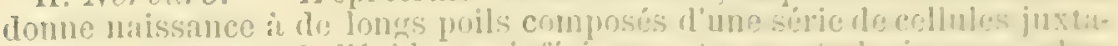
posées. Alu-lessus de l'épilderno inférieur se trouvent plusients conches

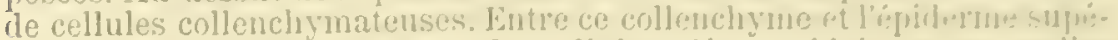

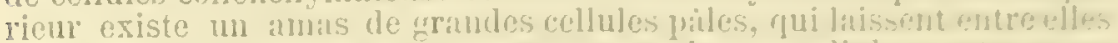
des méats. Polygonales en coupe transversale, ces cellules sont dectun-

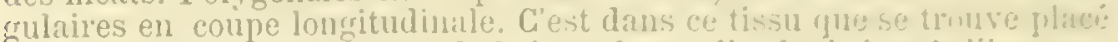

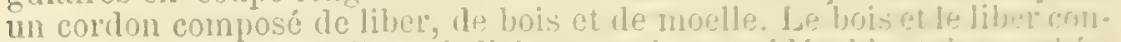

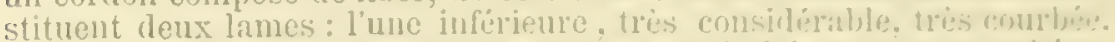
présente une concavité supérieure et ses extremités sont rapproches:

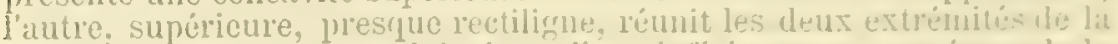

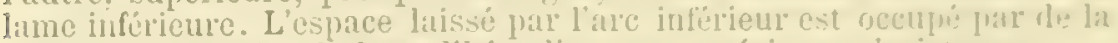
moclle. Quelquefois la lame lihéro-linneuse supérieure n’exist gras.

Le liber est composé à sa surface d'ilots rle fibres ì contour polyomal et a membrane épaissie, limnifiéc et ponctuée ces llots sont supariés les uns des autres par des celiules trés pronctuces et lignifiées. En dodans du liber fibreux siège le liber celluleux dont la puissance est érale it celle du premier.

Le bois est composé de bandes rayonnantes de vaisseaux, séparét: les unes des autres par des rayons médullaires à une ou deux rangées de cellules.

Quant i la moelle, elle est constituée par des cellules d'autant plus considérables qu'elles s'éloignent du bois. Ces cellules ont les paroois ponctuées, possèdent un contour polygonal et sont allongrées parallèlement à l'axe de la nervure.

Houx commun. - Ilex aquifolium. L.

I. Lame interncurale. - L'épiderme de la lame est constitué par une couche de cellules tabulaires it contour polyconal. Ces cellules, it membrane très épaisse, sont recourertes superficiellement d'une forte couche de cuticule qui s'étend sur les parois latérales. Lépilerme, glabre. est pourvu de stomates it la face mférieme seulement. Ces derniers. arrondis, sont entourés de une ou deur couches concentrifues de cellules allongées tangentiellement. Contre l'épiderme supérieur' existe une couche de cellules incolores ì membrane très épaissie (pl. V. tir. I, b), de forme rectangulaire en coupe transrersale. Ces cellules sont plus larges et plus épaisses que celles de l'épiderme arec lesquelles elles al. ternent le plus sourent.

Le parenchyme vert varie suivant la face. Supérieurement, il constitue deux ou trois couches de cellules cylindriques pressees les unes contre les autres, à membrane légèrement épaisse, et disposées en pirlissade (c); ì la face inférieure, les cellules sont rameuses et laissent entre elles des méats et des lacunes (d). Lal plupart ont leur membrane épaissie et ponctuée. On remarque dans ce parenchyme, en général plus développé que le parenchyme supúrieur, des cellules armonies renfermant des cristaux agrglomérés d'oxalate de chaux. Ces éléments cristalligènes se trouvent le plus sourent au voisinage du parenchyme en palissade.

II. Marge. - Contre l'épiderme marginal, doublé de la couche de renfoncement, s'appuie un firisceau d'éléments prosenchymateux it contour polygonal et à membrane lignifiée, ponctuée et très épaissie de manière à ne laisser qu'une faible lumière centrale. 
III. Nervurc. - La nervure est convere supérieurement et inférieurement. Son épiderme est composé d'une couche de cellules tabulaires, qui, vues de face, ont une longueur ne dépassant jamais deux fois la largeur. Contre l'épiderme existe, tant à la face inférieure qu'à la face supérieure, un anas de cellules ì membrane très épaisse, ponctuée. Ces éléments, qui ne laissent entre eux que de faibles méats, ont un contour polygonal, sont allongés parallèlement ì l'axe de la nervure, disposés en file verticale et séparés les uns des autres par des cloisons horizontales. En dedans de l'hypoderme se rencontre un massit puissant de cellules épaissies iz contour arrondi ou polygonal. Ces cellules pâles a menbrane ponctuée, laissent entre elles des méats; quelques-unes contiennent de cristaux agglomérés d'oxalate de chaux. C'est dans ce tissu cellularie que se trouve placé un cordon en forme d'arc. Il se compose inférieurement d'un arc libérien consitué: $1^{\circ}$ par des ilots de fibres arrondies, lignifiées, à parois très épaisses et ponctuées: ¿o par du liber celluleux très puissant. Au.dessus du liber existe un are ligneux concare supérieurement.

Le bois, plus développé que le liber, est formé d’un grand nombra de bandes fibro-vasculaires rayonnantes très rapprochées les unes des autres, et séparées seulement par des rayons ì un seul rang de cellules très applaties tangentiellement. Dans la concavité supérieure laissé par le bois existe un massif de cellules dont les membranes sont épaissies, ponctuées et lignifiées. Ces éléments constituant par leur ensemble une moelle, ont un contour arrondi et polygonal, sont très allongés parallèlement à l'axe de la nervure et séparés les uns des autres par des cloisons horizontales ou obliques. A la partie supérieure de cet amas cellu-

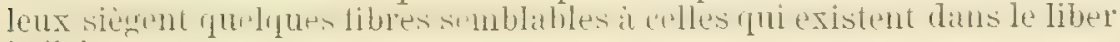
intérieur.

$$
\text { Houx du Paragnay, Maté. - Ilex paraguayensis, Lamb. }
$$

Il se distingne du Houx commun par l'absence de couche cellulaire au-dessous de l'épictorme supérieur et d'umas prosenchymateux à la marge. De plus, le système libéro-ligneux est composé d'un arc inférieur et d'une bande supérieure reliant les deux extrémités de l'arc.

\section{PAMILLES DES PIANTTAGINÉES.}

Grand Plantain. - Plantago major. L.

I. Irme inlerneurale. a. Epiderme. - Une seule assise rle cellules tabulaires a parois minces entre dans la constitution de chacun des épidermes. La membrane des éléments de l'épiderm intérieur présente de nombreuses sinuosités, tandis qu'ia la face supérieure les éléments sont irrégulièrement polygonaux ì parois très peu ondulées. Les deux épidermes sont pourvus de stomates elliptiques disposés sans ordre, de poils ordinaires et de poils glanduleux.

Les poils proprements dits sont coniques, longs ou moyens, composés d'une série unique de cellules superposées ì membrane un peu épaisse et de deux it trois fois plus longues que larges. La cellule basilaire, moins haute mais plus large, nait d'une grosse cellules épidermique dont la surface est arrondie. 
Les poils glanduleux sont unicellulaires, cylindriques, fris conrts: ils

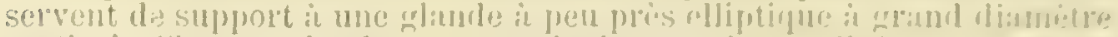

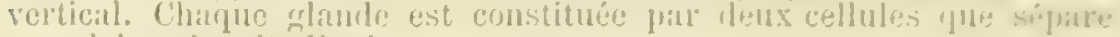
une cloison longitudinals.

b. Mésophylle. - I e in'sophylle vert dépourvu dre collules criatalli-

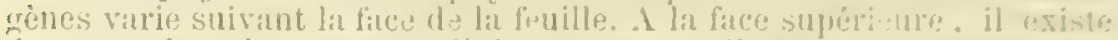
deux couches de grosses cellules courtes, cylindriques. disposeres en

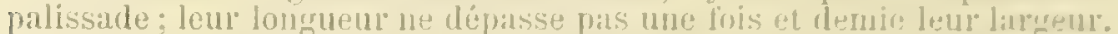
A la face inferieure, le mésopluylle forme trois ou cquatre assiost parralleles de grandes cellules assez pressées, arrondites rou rectanculatres it angles émoussés.

II. Marge. - La marge est uniquement constituéc par rlu parenchyme vert lecouvert d'épiderme.

III. Tervure. - Lat nervure est convere inférieurement, un neu concave supérieurement. Son épiderme est formé de cellules allontrétes un peu épaissies. Il est dépourvu de stomates, más muni de poils cranduleux et de poils ordinaires dont la cellule épideronique qui lui donne nassance a la forme d'une ellipse it mand diametre paralletes a lit nelvure. Au-dessus de l'épiderme supérieur viennent plusieurs couches de collules vertes qui, en section transversale, sont alrondies ou polyur). nales. Au-dessus de l'inférieur existent quelques couches de collenchivme au dedans duquel sière un amas de grandes cellules incolores renfer" mant de lares rrains de chlorophylle. Ces cellules sont polyronales ou in'ondies en section transversale et laissent entre elles de lérers méats.

Presque au centre de la nerrure est situé un filisceau rui est entouré de deux couches concentriques de cellules ì grand diametre timentiel an fuisceau.

Le cordon a la forme d'une ellipse í grand diametre transrerse. If se compose inférieurement de liber, convexe en bas, concave en haut. Ce dernier est constitué ì sa partie infërieure par un arc continu de fibres épaissies, nacrées, collenchymatoïdes, it lumière assez. grande; la portion supérieure est occupée par du liber celluleux.

A lì partie supérieure du liber siège un are ligneux. concave supérieurement.

Le bois, un peu moins épais que le liber, est constitué par des bandes rayomnantes vasculaires assez rapprochées, et séparées les unes des autres par des files de cellules à membrane épaissie. La concarité laissée par le ligneux est remplie par des éléments la plupart très épais , qui. en coupe transversale. ont, en majeure partie, lapparence de fibres li. bériennes. Ce massif est presque entièrement composé par des fibres assez longues ou fibres libériennes trẻs épaisses, nacrées: parmi ces fibres on rencontre des éléments plus petits assez obscurs et semblables ì ceux du liber celluleux.

Plantain moven. - Planlago melia. L.

Le Plantain moyen dillëre du rrand Plantain par l'absence de petits éléments obscurs daus les fibres libériennes et par la présence de cellules ì membranes très sinueuses, aussi bien il lépiderme supérieur qu’à linférieur. 
Plantain Iancéolé. - Plantago lanceolata. L.

Comme le Plantain moyen, le Plantain lancéolé est dépourvu de petits éléments obseurs dans le liber supérieur.

Les denx épidermes ont des cellules peu sinueuses. La plupart des poils, surtout ceux qui existent ì li nervure, sont unicellulaires, très longs, à membrane très épaisse. Quant aux glandes, plus lares, elles ont une forme très diflérente : elles ressemblent à un cône de pin à sommet supérieur. Elles sont multicellulaires et leurs cellules sont sẻparées par des cloisons dirigées en tous sens (pl. III, fig. III).

FAMILLE DES VERBÉNACÉES.

Fenille de Verreine ofricinale. - Terbena officinalis. L.

I. Lame interneurale. a. Eniderme. - L'épiderme. glabre, muni de stomates et de glandes sur les deux filces, est formé d'une assise de cellules tabulaires. Les éléments de l'épiderme inférieur sont très sinueux, tandis que ceux du supérieur sont irréguliẻrement polygonaux ou présentent de faibles ondulations.

Les stomates elliptiques sont placés entre quelques cellules épidermiques.

Les glandes, plus nombreuses sur la face inférieure que sur la face supérieure, sont situẻes dans une dépression de l'épiderme. Elles sont arrondies, et composées de $4-8$ cellules disposées sur un même plan horizontal et séparées entre elles par des cloisons verticales. Ces glandes sont supportées par' un pédicelle unicellulaire très court.

b. Mésophylle. - Le mésophylle dépourvu de cellules cristalligènes est hétérogène. A la face :...jérieure existent trois couches parallèles de grandes cellules cylindriques dont les superficielles sont d'ordinaire juatre fois plus longues que larges. Au-dessous du parenchyme en palissade sont des cellnles arrondies ou rameuses dirigées en tous sens ef laissint entre elles de nombreux méats.

II. Nervure. - L'épiderme a les cellules allongées parallèlement à l'ase de la nervure; il est dépourvu de stomates, mais donne naissance i des poils ordinaires et ì des poils glanduleux.

Les glandes ressemblent it celles qui sont sur l'eppiderme interneural.

Les poils sont unicellulaires. Ils ont la forme d'un cone dont la lirse courbe est logée dans une dépression située au sommet d'une proéminence conique et multicellulaire de l'épiderme.

Lile ou trois assises de collenchyme sont en contact avec l'épiderme supérieur et a vec l'épiderme inférieur. Une masse parenchymateuse, dans Iarquelle sière un cordon, est placée entre les deux collenchymes. Les cellules grandes et incolores ont leurs parois minces. Elles laissent entre elles des ménts. et ont la forme de polygones assez régulier's lor'squ'on examine une coupe transversale de la ner'vure.

Le cordon, dont la section transversale a l'aspect d'un croissant, est composé de liber, de bois et de parenchyme médullaire.

Le liber forme it sal partie inférieure un are continu de fibres dont les membranes peu épaissies sont cellulosiques. A sa partie supérieure il est celluleux. 
Le bois est composé d'un frand nombre de bandes, féendieres et patralleles de gros vaisseanx spinanx, ponctues, traches.

Le bois presente in sa partif superieure uno concavitis remplio par ap massif cellulate dont les áments asse\% petits ont lat forme de longs prismes droits it base polyngnale.

\section{Lippia citronelle. Citronclle. - Limpia cilviollora. hunth.}

1. Lame internetrale. a. Fipiderme. - Lëpiderme infërieur forme une seule assise de cellules tabulatres et polyconales supredicidlenent. Elles sont revêtues dune conche assez épaisse de cuticulr, qui, exumi. nẻe de fitce, semble striée. Cétépiderme, pourvu de stomates elliptigues placés sans ordre, est hérissé à la face supérieure de poils unicellu. laires, coniques, recourbés dès la base, poils dont les parois sont par. semées de petits tubercules. Il produit, en outre, de frosses grandes unicellulaires ou pluricellulaires á éléments disposés sur un plan horizontal ; ces glandes, presq:2e sphériques. sont sessiles on portées sur un pédicelle unicellulaile extrêmement court.

L'épiderme supérieur, dépourvu de stomates, est composé de cellules plus petites rui sont ì peine crètées superficiellement.

b. Mésophylle. - Le mésophylle chlorophyllien présente cette particularité d'aroil tous ces éléments assez serrés et allongés perpendi. culairement a la surface foliaire. A la face supérienre, existent deux couches de cellules cylindriques disposées en palissade. Au-dessous d'elles viennent des cellules allongées dans le mème sens, qui ont la forme d'un biscuit ou ofrent des membranes légèrement sinueuses. Ces éléments ne laissent entre eux que de trés petits méats. Le mésophylle ne renferment point de cellules cristalligènes.

II. Marye. - La marge est formée par l'extrémité du mésophylle recouverte d'épiderme.

III. Nervore. - L'épiderme glanduleux et poilu a les éléments épaissis, allongés suivant la nervure. En dedans de lui se trouve du collenchyme, dont les cellules, épaissies sur tout leur pourtour et un peu ponctuées, ont la forme de longs prismes it base polygonale. Le collenchyme est moins épais, mais plus déreloppé latếralement ì la face inférieure. A la face supérieure, ils forment un amas dont la section transversale est quadrangulaire. Contre les éléments callenchymateux inférieurs s'appuie une masse de cellules ì membrane mince. laissant entre elles des méats. Sur une coupe transversale, ces cellules paraissent polygonales, tandis qu'elles sont rectangulaires dans le sens longitudinal. Au-dessus de ce parenchyme, on troure, en gramant le collenchyme supérieur :

10 Un arc de fibres libériennes, le plus sourent lignifiées et épaissies:

$2^{\circ}$ Du liber celluleux, généralement égal en puissance au liber fibreux;

$3^{\circ}$ Un arc lignifié, dont les éléments sont disposẻs en séries rayonnantes, séparées par des rayons ì une ou deux rangées decollules minces ;

$4^{\circ}$ Quelques cellules i parois cellulosiques pressées les unescontre les autres et de forme prismatique it base polygonale ;

$5^{\circ}$ Deux ou trois couches de cellules assez grandes it membrane le plus souvent épaissie, très ponctuée et lignifiée; 
$6^{\circ}$ Un ou deux groupes de liber interne qui offre les mêmes éléments que l'externe:

$7_{0}$ Un amas de cellules le plus souvent lignifiées et ponctuées sem. blables à celles qui existent au-dessous du liber supérieur.

Ce massif s'appuye contre le collenchyme supérieur.

\title{
FAMILLE DES LABIÉES
}

\begin{abstract}
Especes: Sauge oflicinale. Salria officinalis. L. - Sauge des prés. S. pratensis. L. Sauge slarée. S. sclarea. L. - Romarin officinal. Rosmarinus officinalis. L. - Mélisse othicinale. Melissa officinalis. L. - Nepeta cataire. Nepeta cataria. L. - Calament oflicinal. Calamintha officinalis. Moensch. - Bétoine officinale. Betonica officinalis. L. - Marrube commun. Harrubium rulgare. L. - Gléchome lierreterrestre. Glechoma hederdeca. L. - Hysope oflicinal. Iyssopnes officinalis. L. Origan dictame. Origanum dictamnus. L. - Sarriette des jardins. Satureia hortensis. L. - Thym commun. Thymus vulgaris. L. - Thym serpolet. Thymus serpyllum. L. - Bugle rampant. Ajuga reptans. L. - Germandree petit chéne. Teucrium chamcedrys. L. - Germandrée aquatique. Teucrium scordium, L.
\end{abstract}

I. Lame interneurale. - Les cellules épidermiques sont tabulaires. Leur contour est sinueux. (Melissa, Salvia pratensis. L. S. sclarea. L.) ou polygonal, (Salvia oflicinalis. L.). Leur paroi est tantôt mince (Melissa), tantôt épaisse et ponctuée (Thymus).

L'épiderme produit des stomates à la face inférieure seulement dans Melissa, Nepela, Calamintha, Glechoma, Rosmarinus, Origanum, Marmbium, Tencrium scondium L. Il y en a au contraire aux deux faces dans le T'eucrium chamadrys, les Salvia, l'Hyssopus, l'Ajuga, la Betonica, la Satureia, le Thymus.

Les strmates, ordinairement elliptiques, sont, dans un grand nombre d'espèces, placés perpendiculairement au milieu de cloisons séparant deux cellules épidermiques (pl. I, fig. VI). Nous n'avons trouvé d'exception que dans le Marrubium vilgare et le Teucrim. Dans ces deux espèces, la plupart des stomates sont situés entre trois ou quatre cellules de l'épiderme.

Les poils sont unicellulaires ou composés d'une série d'articles superposẻs. Rameux dans le Rosmarimes (pl. II, fig. IV), et l'Origamm dictammes, ils sont simples dans les autres espèces étudiées. Ils sont courts dans la Melissa, l'Hyssopus la Satureia, le Teucrium chamcedrys. L. et le Thymms. Ils sont formés dans ces plantes de une it trois cellules. Les poils de liz Melissa et de l'Hyssopus sont droits; ceux de la Satureia, sont gros et recourbés en forme d'ergot. La cellule supérieure de la plupart des poils du Tencrium chamadrys. L. et du Thummus forment avec la cellule voisine un coude très prononcé. La Betonica présente de longs poils unisériẻs, dont les cellules allongées sont renflees i leurs deux extrémités Dans le Glechoma, ces appendices sont supportés par une éminence pluricellulaire et conique de l'épiderme (pl. II, fig. I).

On rencontre sur les deux faces de la feuille du Marmuium des poils dont plusieurs s'agglomèrent au nombre de deux, trois ou quatre sur une proéminence commune.

La paroi des cellules du poil est tantôt lisse (Salvia pratensis. L.), tantôt hérissée à sa surface externe de tubercules arrondis (Hyssopus, Thymus). 


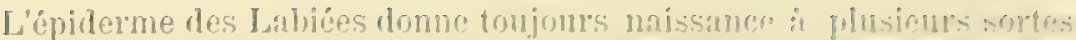

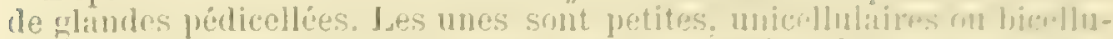

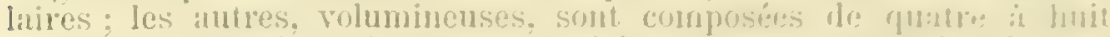

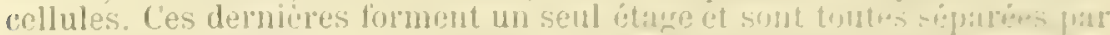
des cloisons verticales. lee pédicelle qui les soutient ent rompt. unicrallu-

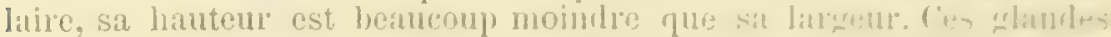
sont souvent placées dans iles fossettes produites par l'éphlerme. I.

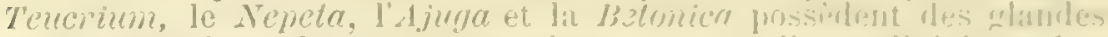

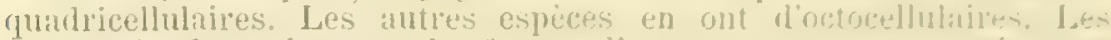

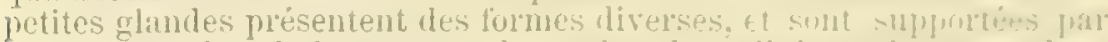
un pédicelle dont la longueur et le nombre des cellules qui untrent din: leur constitution varient avec les espices.

Les glandes sont splrériques dans la Melissr, ovoüles dans le Corne mintha et l'Origanmen.

Le pédicelle est court, unicellulaire, dans la Melisso, le dépétre. Ie Glechoma, bicellulaire dans le Rosmainus.

Certaines glandes, que l'on rencontre sur la nervure des Salria et du Marrubium, ont un pédicelle composé de deux cellules dont l'inférieur longue à la forme d'un cône à sommet arrondi, tanclis que la suptrieure. cylindrique, peu élerée, a sa base moins large que le sommet dé la cellule inférieure.

Le Teucrim scoirlium possede des glandes dont le support est lonr et composé de quatre it six cellules.

b. Mísophylle. - Le mésophylle est toujours dépourru de cellules cristalligènes. Au-dessous de l'épiderme supéricur du hósinrrimus est me assise de cellules incolores: dams les antres espices. il nit y ante les deux épidermes que des cellules it chlorophylle.

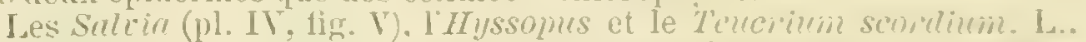
ont toutes leurs cellules dirigées perpendiculairement à lia suifice foliaire. Leurs éléments nont pas la mème longueur, ils diminuent de la face supérjeure à la face inférieure. Un tissu supérieur en palissade et un inférieur rameux existent dans les autres plantes. La Melissn, le Calamintha, la Saturia et le Nenctr possident me couche de tissu muriforme. Le Thymus, le Glechoma. l'iluga en out deur ou plusieurs.

II. Marge. - La termison latérale du mésophylle et de l'épiderme constitue la marge.

III. Nerume. - Dans l'intérieur de la nerrure existe un are libéloligneux plus ou moins étendu. Cet arc est composé inférieurement de liber cellulo-fibreux. Les fibres sont cellulosiques, it l'exception ale celles du Rosmarimes qui sont sourent lignifiées; elles forment un arc continu. Le bois situé au dessus du liber est constitué par plusieurs bandes rayonnantes de vaisseaux. A la partie supérieure du bois siège un amas de cellules allongées en forme de prisme droit a base polvgonale. Ces cellules ont leurs parois nacrées et épaissies surtout aux angles. 'Tout le systeme que je riens de signaler est enreloppé par de grandes cellules pâles qui touchent a la partie supérieure et inférieure de la nervure du collenchyme plus ou moins dévelopné. Certaines espéces sont dẻpourrues de tissu collenchymateur.

Le Rosmarimus possède au-dessus et au-dessous du filiscean un amas homogène de cellules dont les membranes sont épaissies. Il en est de mème du Thumus sermyllum; mais le Thymus vulyaris présente à la

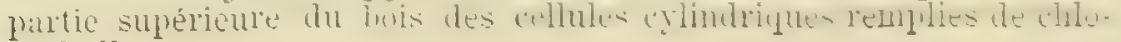
rophylle. 
Caractères commens: Poils glanduleux ì glandes unicellulaires ou bicellulaires toujours mélangẻes à de grosses glandes au moins quadricellulaires. Poils ordinaires. Point de glandes internes. Point de cellules cristalligènes. Point de canaux sécréteurs. Point de liber interne en îlots.

Sauge officinale. - Salvia officinatis. L.

I. Lame intemerrale. a. Expiderme. - L'épiderme est formé de cellules tabulaires it surface irrégulièrement polygonale. Il possède aux deux faces des stomates, des poils et des glandes. Les stomates elliptiques sont placés au milieu des parois séparant deux cellules.

Les poils fins, d'un diamètre presque égal sont ordinairement composés de deux cellules allongées et superposées dont la plus longue est celle du sommet. Leur surface est ordinairement chagrinée. Les glandes sont arrondies, unicellulaires, bicellulaires ou pluricellulaires; dans ce cas, les cloisons de séparation sont verticales. Le pédicelle qui supporte chaque glande est variable. Le plus souvent il est cylindrique, composé de une ou deux cellules égales en diamètre et en hateur; mais au milieu de ces glandes ainsi pédicellées on en trouve dont le poil est constitué par deux cellules inégales: l'inférieure, deux à trois fois plus haute que la supérieure, a la forme d'un trone de cône dont la grande base tient à l'épiderme. La supérieure est plus petite, plus large que haute.

b. Mésophylle. - Le mésophylle est toujour's coloré en vert par de la chlorophylle. Les éléments qui le composent sont presque tous allongés et dirigés perpendiculairement ì l'épiderme (pl. IV, fig. V). A la face supérieure, ils forment deux à trois couches et sont cylindriques. A la face inférieure, ils deviemnent arrondis. Ces éléments diminuent de hauteur de l'épiderme supérieur à l'épiderme inférlieur.

II. Nervure. - L'épiderme a des cellules allongées parallèlement à la nervure, leur membrane épaissie ne présente point de ponctuations ; il est muni de poils et de glandes analogues a celles que l'on rencontre sur la base, seulement les glandes à pédicelle moyen sont plus considérables.

En dedans de l'épiderme, siègent deux ì trois couches de cellules collenchymateuses à contour épaissi. Cet hypoderme enveloppe une masse de cellules a membrane assez mince, polygonales dans le sens transversal; carrées ou rect. nrulaires dans le sens longitudinal. Ces cellules, ordinairement incolores, laissent entre elles de petits méats, et aucune d'elles ne contient de cristaux d'oxalate. C'est au milieu de ce parenchyme que se trouve le système libéro-ligneux qui a la forme d'un croissant à concavité supérieure.

Le liber qui existe à la partie inférieure est constitué à sa surface par un amas de fibres cellulosiques, blanches, épaissies, à section transversale polygonale. Cet arc fibreux enveloppe le liber celluleux assez développé.

Le bois est formé de plusieur's lames rayonnantes fibro-vasculaires assez rapprochées. Les trachées plongent un peu dans un petit massif situé dans la concavité du bois. Ces cellules, pressées les unes contre les autres, un peu épaissies, ont la forme d'un long prisme droit à base polygonale. 


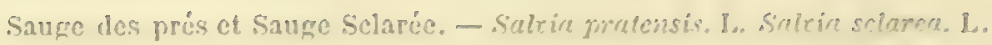

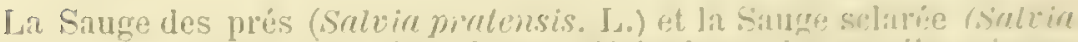

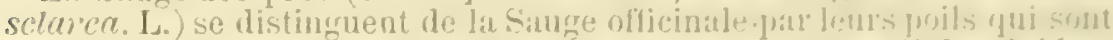

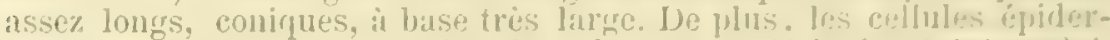

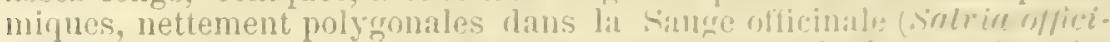
malis. L.), ont leurs parois simucuses en Saure sclance (Solvio soluien. L.) et des prés (Salria piolensis. 1.).

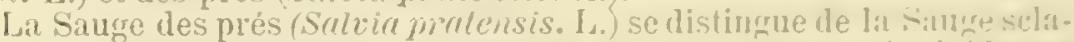
lie (Salviu sclaren. L.) par la présence de ponctnations tries éviluntos sur les membranes des cellules de l'épiderme infëriem do la mervure et par des stries apparentes sur la surface le cet épiderme, stries dues it des crêtes formécs par la cuticule.

La Sauge des prés peut être substituée au Matico.

Feuille de Romarin ofticinal. - Rosmarines officinalis. L.

I. Lame internenare. - Lépiderme constitue sul les deux faces de la feuille une assise de cellules tabulaires recourertes sujerficiellement l'une cuticule assez épaisse. Les cellules de l'épidelme inferien ont un contour sinueux, tandis que celles du supérieur sont polygonales.

De nombreux stomates, et une quantite considérable de poils existent ila face inférieure seulement; mais on rencontre des glandes sur. les deux membranes épideriniques.

Chaque stomate a la forme d'une ellipse et sc troure sur une paroi intermédiaire a deus cellules. Les poils sont pluricellulaires. Ils sont composés d'une file verticale d'articles d'où partent de distance en distance de courtes branches coniques. unicellulaires (pl. II. Iig. IT).

Certaines glandes formées d'une cellule sphérique sont soutenues par un pédicelle bicellulaire dont la cellule basilaire est généralement un peu plus longue que large, tandis que la supérieure est moins haute. D'autres crlandes sont composées de quatre cellules disposées en croix et séparées par des cloisons verticales. Les autres enfin ont luuit cellules rangées sur un plan horizonta!. Les deur dernières especes surmontent toujours un poil unicellulaire qui a l'aspect d'un cylindre tre"s surbaissé.

Une ou deux assises de cellules incolores seitendent au-dessous de l'épiderme supéricur'. Ces couches ne sont pas toujour's contintres; il ý a des espaces où elles n'existent point et alol's le tissu situé plus bas est directement en contact arec l'épiderme. Ces assises sont les prolongements latéraux d'ume masse cellulaile qui se troure au-dessus des faisceaux libéro-ligneux.

Le parenchyme vert forme il la face supérieure de la feuille deus il trois couches de grandes cellules cylindriques, perpendiculaires it l'épiderme et trois a six fois plus longues que lirges. Au-dessons d'elles siègent des cellules qui contiennent quelques grains de chlorophrlle, sont irógulières, raneuses, et laissent entre elles des méats.

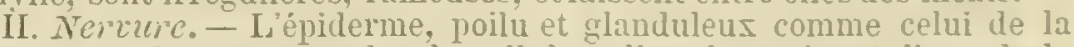
lame, coustitue une couche de cellules allongées suirant lase de la nelvule.

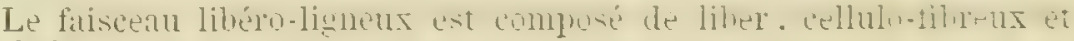
de bois. 
Le liber a la forme d'un arc, concave supérieurement; sa partie inféricure est prosenchymateuse, tandis que la supérieure est cellulaire.

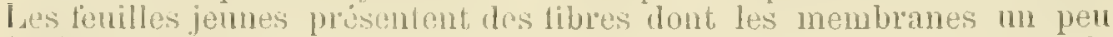
épatissies sont cellulosiques, tandis que les ârées en out dont les parois sont limiliées.

Le bois forme des bandes peu nombreuses de vaisseaux, spiraux, ponctués et de trachées.

Au-dessous du cordon libéro-linneux existent quelques assises de grandes cellules incolores, qui laissent entre elles des méats et ont l'aspect de longs eylindres ou de prismes droits à base polygonale. Entre ce tissu et l'épiderme inférienr siège du collenchyme.

Contre le bois et ì la partie supúrieure s'appuient deux couches de petites cellules cylindriques au-dessus desquelles est un amas quadrangulaire à grand còté perpendiculaire ì la surfice de la feuille. Cet amas est composé de grandes cellules cylindriques ou prismatiques dont les parois sont épaissies.

\section{Suhstitutions.}

On peut confondre le Romarin avec l'Andromède à feuille de polium (Andromedn poliffolia. L.), et avec le Ledon des marais (Ledum $p \alpha$ lustre. L.). Voici les caractères histologiques qui permettent de reconnaitre ces espèces.

L'épiderme de l'Andromède (Andromeda poliifolia. L.) est dépourvu de poils et de glandes. Il n'existe jamais d'assise de cellules incolores audessous de l'épiderme inférieur. La section transversale du faisceau libéroligneux a la forme d'un triangle. Ce cordon possède au-dessus du bois un groupe d'éléments dont les parois très épaisses sont lignifiées. Enfin le parenchyme neural contient des cellules cristalligènes.

Le Ledon des marais (Ledum prulustre. L.) possède des poils non ramifiés et des glandes dont le pédicelle est souvent composé d'une ou deux séries de trois à cinq cellules. Des cellules cristalligènes se rencontrent dans le parenchyme vert de la lame et dans la nervure.

\section{Mélisse officinale. - Melissa offeinalis. L.}

I. Lame intemeurale. a. Epiderme. - Une seule couche de cellules constitue l'épiderme. Ces éléments tabulaires présentent superficiellement une membrane mince plissẻe.

L'épiderme pourvu de stomates à la face inférieure seulement donne naissance à des poils ordinaires et à des poils glanduleux. Les stomates ellipliques siègent sur la paroi de séparation de deux cellules épidermiques voisines. Les poils sont presque tous unicellulaires sur la lame. Ils sont coniques, courts, droits, une a trois fois plus longs que larges. Leur membrane, de médiocre épaisseur, est garnie de petits tubercules qui donnent ì la surface un aspect chagriné.

On trouve plusieurs sortes de glandes sur l'épiderme. Toutes sont sphériques et supportées par-des poils extrêmement courts unicellulaires ou quelquefois bicellulaires (pl. V, fig. II). Les unes sont composées d'une seule cellule, les autres de deux cellules séparées par une cloison verticale; les plus grosses, multicellulaires, sont formées d'une couche horizontale de huit cellules, séparées par des cloisons verticales. 
1) Mesophllle. - I,e mesroplyylle est vert, heterorgine, ret no firoduit point de cellules cristalligenes. I la lace superieure criste une seule couche de cellules eylindrirgues (b), pressees les unes contre les autres, troin is quatre tois plus longues rque larges et dirjeres perpendiculairement : l'epiderme.

A la face inférieure se trouvent deux a trois couches parallies de collules rameuses (c) qui, sur une coupe transversale, paraissent arrondips ou rectangulaires. Ces leux ou trois assises fornent ensemble une lamc toujours moins dévelopjée que le parenchyme en palissade.

II. Maire. - Ia marge est uniquement constituée par la terminaison latérale du mésophylle et de l'épiderme.

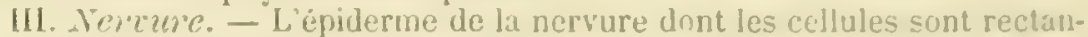
gulaires ou polyédriques, allongees dans lú sens de la nerrure. presente comme celui de la lame des poils ordinaires et des poils glanduleux: seu. lement, outre les poils courts unicellulaires, on en trourc de julus lonis. composés d'une série de cellules superposies. Én dedans de l'épiderne se trouvent deux a trois assises de cellules collenchymateuses, dont la membrane épaissie ne présente que tres peu de ponctuations. Elles ont la forme d'un long prisine droit ì base polygonale.

Contre ce collenchyme s'appuie un massif assez considérable de grandes cullules dans lequel est plongé un arc. en majeure partie libéro-ligneux. Ces éléments, qui constituent la masse cellulaire, ont les membranes assez. minces et laissent entre eux de faibles méats, ont la forme d'un prisme is base polygonale. L’arc libéro-ligneux. très étendu . est constitué en allant du haut vers le has de la nervure: 10 de liber dontles éléments. tous cellulosiques, ont leur membrane un peu épaissie, nacrée; ¿n de bois constitué par plusieurs bandes rayonnantes de quatre i six vaisseaux ponctués, trachées, séparées par des rayons de un à quatre rangs de cellules. Dans lit concavité supérieure formée par le bois on trouve un petit amas de cellules à membrane un peu éłaissie. nacrée. Ces éléments polrsonaux en couje transversale, sont rectangulaires, très allongés dans le sens longiludinal.

$$
\text { Sépeta cataire. - Vepela calaria. L. }
$$

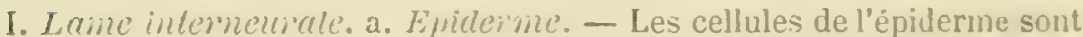
talulaires; leur contour est trés sinueux. Les deux épidermes produisent des poils et des glandes t tandis que l'inférieur possède seul des stomates. Ceux-ci sont elliptiques. Chacun d'eux est placé perpendiculairement au milieu d'une paroi limitant deux cellules épidermiques.

Les poils ont la forme de còne allongé. Ils sont composés d'unc tile lonvitudirale de trois a six aricles, ordinarement quatre it sept fois plus longs que larges. Leur membrane est tantüt lisse, lantot hérissée i sa surface externe de pelits tubercules.

On rencontre trois espèces de glandes sur l'épiderme. Les unes sont unicellulaires, sphériques ou ovoïdes à petite extrémité supérieure; d'autres sont forr.ées de deux cellules séparées par une cloison verticale.

Ces deux sortes de glandes surmontent un pédicelle unicellulaire qui est généralement plus large que haut, et a l'aspect d'un tronc da cône à pelıte hase insérée sur l'épiderme. Les glandes de la troisième espèce sont constitués par quatre cellules placées sur un même plan horizontal et disposées en croix.

b. Mésophylle. - Le parenchyme en palissade est représentẻ par ume 
assise de cellules cylindriques, vertes, perpendiculaires à la surface de la feuille et dont la longueur dépasse trois à quatre fois la largeur. Au-dessous siègent trois à quatre couches de cellules aplaties de haut en bas et allongées parallèlement à l'épiderme. Ces éléments laissent entre eux des méats assez peu considérubles.

Il n'existe point de glandes ni de cellules cristalligènes dans le mésophylle.

II. Yervure. - A l'exception des poils de l'épiderme, qui ressemblent à ceux de la lame, les tissus de la nervure du Yépéta offrent les mêmes caractères que ceux de la Mélisse.

Calament ofticinal. - Calamintha ofricinalis. L.

1. Lame inlemennale. a. Epidemue. - Les cellules de l'épiderme inférieur possèdent un contour très sinueux, tandis que celles du supérieur offrent de très faibles ondulations. L'épiderme inférieur produit seul des stomates et des glandes; mais il existe des poils ordinaires aux deux faces.

Chaque stomate est elliptique et se trouve sur une cloison courbe qui sépare deux cellules épidermiques.

Les glandes sont les unes unicellulaires, les autres composées de huit cellules placées sur un même plan horizontal. Les premières ont la forme d'une massue à grosse extrémité supérieure. Un pédicelle court et unicellulaire sert de support aux deux sortes de glandes.

Les poils ordinaires, assez communs sur la face inférieure, sont plus rares sur la supérieure. Ceux de l'inférieure sont presque tous formés d'une file de quatre à six cellules très longues, dont, les membranes sont chargées à l'extérieur de tubercules coniques. Les poils de l'épiderme supérieur sont la plupart unicellulaires. Ces derniers naissent du centre de la paroi superficielle d'une cellule épidermique plus grande que leur base.

1). Mésopllylle. - Contre l'épiderme supérieur s'appuie une assise de grandes cellules cylindriques, perpendiculaires it la surface de la feuille, et trois ì cinq fois plus longues que larges. Au-dessous d'elle est une lame d'égale puissance, composée de petites cellules arrondies ou irrégulières. Toutes les cellules du mésopylle renferment de la chlorophylle, aucune n'olfre de cristaux dans leur cavité.

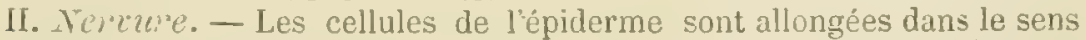
longitudinal de la nervure. L'épiderme donne naissance à des glandes et à des poils dont quelques-unes ont une base enchassẻe dans une proéminence conique pluricellulaire.

Entre les deux membranes épidermiques so trouvent du collenchyme, du parenchyme méatique et un faisceau libẻro-ligneux.

Le collenchyme forme trois couches.

Les cellules du parenchyme sont cylindriques ou prismatiques à base polygonale, et sont incolores. Leur paroi est assez mince.

Le liber a la forme d'un arc continu. La paroi de ses éléments présente les réactions de la cellulose.

Le bois est constitué par plusieurs lames inféro-supérieures et parallèles de vaisseaux.

Thym commun. - Thymus vulgaris. L.

I. Lame intemenale. a. Epideme. - Les cellules épidermiques sont tabulaires. Leur contour est polygonal ou légèrement sinueux, 


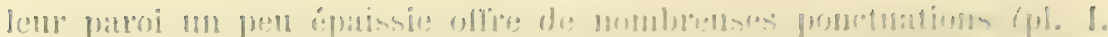

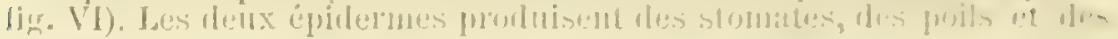
mlandes.

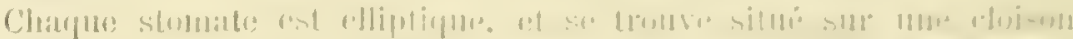

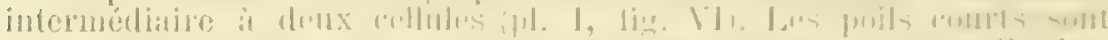

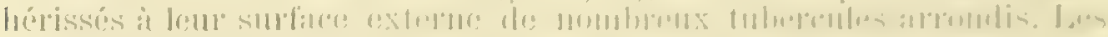

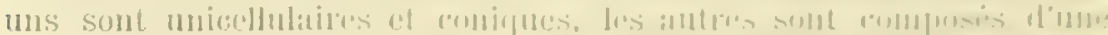

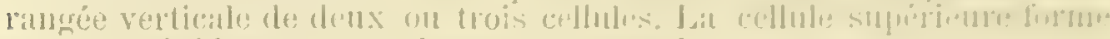
avec ba précédente un condule assise pronesuer.

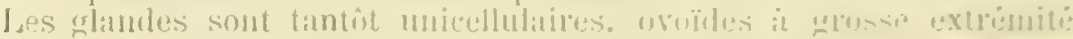

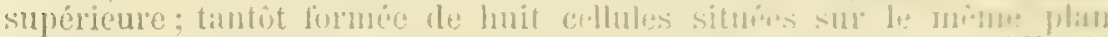

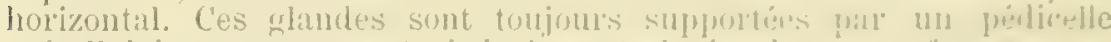

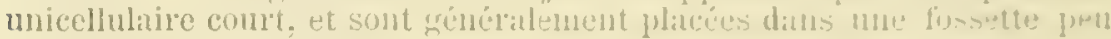
profonte de l'épiderme.

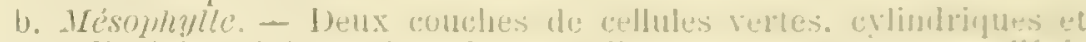

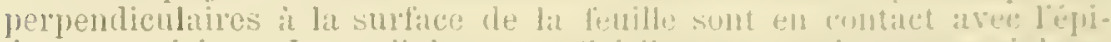

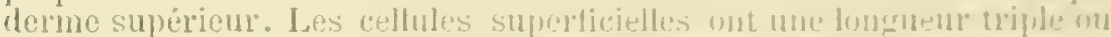

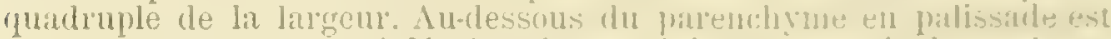
une lame presque aussi. développée que lui et cinnposée de quelques conches de cellules sphúrinues, ovoüdes ou cllipsolilales.

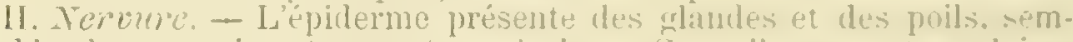
bablos in cenx qui se trouront sul la lame. Ses collues. lectanmulares lorsqu'on les eximine de fice. sont allomgés suivant lit nervore. A lat farce inférieure de l'épirlerme supériente sierent doux couches de cellutes vertes (fui se continuent latéruenont avec lo tissu uniforne de lit

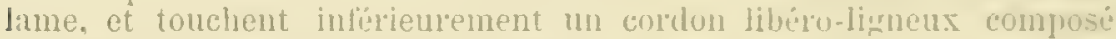
d'un are de liber" et d'mu al\%: de bois

Le libel" ollie ¿̀ sa périphérie des fibres épaissies à membrane cellulosique.

Le bois est folmé de quelques bandes de valiseaux ponctués, spintax et de tracheses.

Au-dessous du faiscenu existe deux it trois assises de cellules incolones allongeses, et ayant lit folme de eylindre ou de prisme droit dont lat base est polygonale.

Thym serpolet. - Thininus scryyllum. L.

So distugrue du précédent, en ce yue la base de la teuille porte il ses bords de longs poils coniques dont les plus grands sont composis dine stéle de cing a six cellules. De plus. l'épiderme du serpolet produit de rares poils, presque tous tris courts et unicellulaires.

\section{Hysope oficinal. - Hyssopres officinalis. L.}

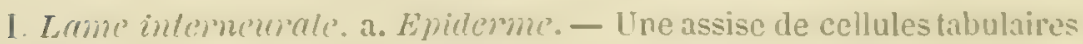
constitue l'épiderme. Ces cellules présentent une surface irrégulierement polyonale ou un contour faiblement sinueux.

On rencontre aux deux faces des stomates, des poils ordinaires et iles glandes pédicellées.

Les stomates elliptiques sont situés au milieu de parois courbes sẻparant deux cellules épidermiques et sont perpendiculaires à ces membranes. Les puils courts et coniques sont les uns unicellulaires, les autres complosés 
l'une rangcee de deux ou trois cellules. Leur surface extérieure offre de nombreux tubercules.

Les glandes sınt toujours supportées par un pédicelle cylindrique très court dont la longueur ne dépasse pas la largeur. Les unes, petites, sont globuleuses, unicullulares ou bicellulaires: dans ce dernior cas les deux cellules sont séparcees par une cloison verticale. Les autres, volumineuses, sont composées de huit cellules toutes placées sur un mème plan horizontil.

1. Mésonhylle. - Les cellules du mésophylle renferment toutes de li chlorophylle; aucune ne contient des cristaux Elles sont toutes dirigées perpendiculairement à la surface de la feuille, seulement leur longueur diminue de la face supérieure à la face infétieure. Les cellules supérieures sont cylindriques, deux i trois fois phis longues que larges, tandis que celles rqui avoisinent l'épiderme inféricur sont sphériques ou ellipsoïdales.

II. A 'rver'e. - L'épilerme dëpoursu de stomates, est poilu et glanduleux. Sis cellules sont allongées dans le sens longitudinal de la nervure et oflient des parois faiblement épaissies mais ponctućes.

Dans l'intérieur de la nervure existe un cordon libéro-ligneux composé a sa partic inférieur d'un are de liher dont tous les éléments présentent une membrane constituće chimiqnement par de la cellulose. Au-dessus du Jiber siège un are ligneux formé de plusieurs bandes parallèles de vaisseaux ponclués, spiraux, et de trachées.

Ce cordon est enveloppé par du parenchyme dont les éléments sont plus nombreux et plus gros a la face inférieure qu'à la face supérieure de la nervure. Ils sont incolores, laissent entre eux de petits méats et ont la forme de cylindres ou de prismes courts i base polygonale. Ce tissu touche à sa périphérie quelques couches de collenchyme qui est en contact avec l'épiderme.

\section{Sarriette des jarlins. - Satureia hortensis. L.}

1. Lrme inteincurale. a. Epiderme. - Les cellules épidermiques sont tabulaires et ont un contour très sinneux. Des stomates, des poils ordinaires ot glanduleux existent aux deux faces. Les stomates sont elliptiques et placés perpendiculairement sur une cloison de séparation de deux cellules de l'épiderme.

Les poils courts et coniques sont unicellulaires ou bicellulaires. Leurs parois sont munies de petits tubereules. Les glandes sont unicellulaires ou pluricellulaires. Les premières sont petites et sphériques; les secondes, volumineuses, sont composées d'une rangée horizontale de huit cellules.

b. Mésophlylle. - Au-clessus de l'épiderme supéricur est une assise de grandes cellules cylindriques, vertes, perpendiculaires à la surface foliaire et trois à quatre fois plus longues que larges. Ce tissu est en contact it sa partie inférieure avec une lame cellılaire yui lui est égale en puissance. Cette laine est constituée par des cellules irrégılières, dirigées en tous sens et laissant entre elles de nombreux méats.

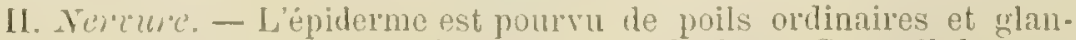
dulcux, semblables it ceux qui existent sur lit lame. Ses cellules sont allongées snivant lix: de lir nervure. Entre les deux épidermes, est mterposé du parleachyme dont les cellules pailes, polygonales sur une coupe thansversale, rectangulaires sur une coupe longitudinale, laissent entre elles de petits méats et ofrrent des parois assez zuinces. Ces cellules 


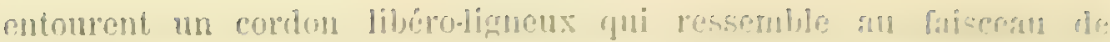
l'lysope.

Isugle rampant. - liugre goyentus. I.

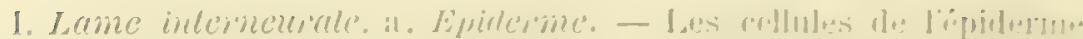

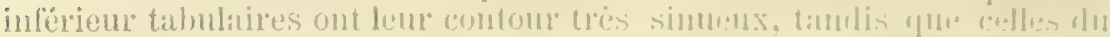

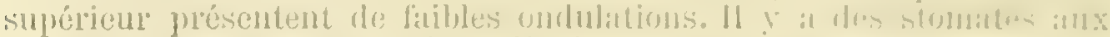
deux laces ansi que des poils ondinatires ot des ulamles.

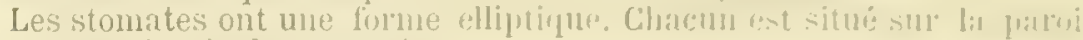
intermédiaire de denx cellules.

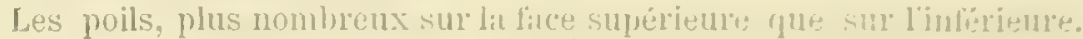
sont assez loness, coniques et composés d'une lile rle guatre it six cel-

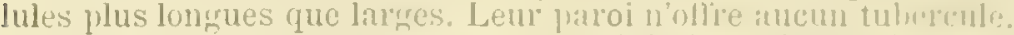

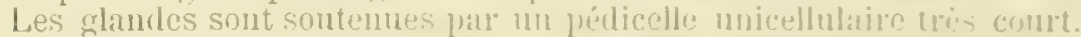
Les unes sont bicellulaires, les autres quadricollulates. Lansilon les examine de sommet elles paraissent almonlies. Cos flandes snut enloncées dans des dépressions épidermiques.

b. Mésopholle. - I.e parenchyme en palissade est composis de deux

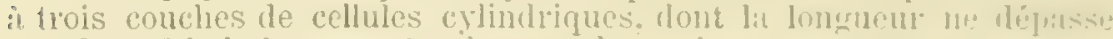
pas denx fois la largenr. Au-dessons de ce tissu, se tronbent quelpuex assises paralleles de cellules qui, sur une coupe transiersile. sinhlint clliptiques ou rectangulaires, allongées parallèlement it lépiderme. ct sont rameuses de face. Elles laissent entre elles des méats.

II. Nervore. - L'épiderme est poilu et findulleux. it cellules allongées, munies de parois ponctuées. Les deux épidermes survent ile limite extériente à du parenchyme méatique rentermant un conton libéro-lignenx.

Les cellules du parenchyme ont la forme de prismes it base polyounale. Leur membrane est mince.

Le liber constitue un are d'éléments, dont les parois sont un peu épaissies et cellulosiques.

Le bois est composé de plusieurs lames paralleles, inféro-supérieures de vaisseaux ponctués, spiraux et de trachées.

Bétoine ofticinale. - Betonica ofricinalis. L.

I. Lame intemenurale. a. Epideime. - Les cellules ipiderniques, tabulaires, présentent un contour irrigulier, sinueux, mais les sinuosités snnt pel prononcées. Les stomates situés aux deux faces sont elliptiques, on les rencontre sur des parois séparant chacune deux cellules de l'épiderme.

Il y a aussi sur les deux facies des poils ordinaires ct glanduleux. Les premiers, longs et coniques, sont composés de cellules cylindriques allongées, renflées à leurs extrẻmités; il n'y a d'exception que pour la cellule terminale, effilée en pointe; mais celle-ci présente le renflement ì sa Lase. La base du poil a la forme d'un còne à sommet inférieur ; elle est enfoncée dans l'épiderme.

Les glandes sont bicellulaires et quadricellulaires. I.eur péticelle. peu élevé, est cylindrique.

b. Mésollunlle. - Si l'on fait une coupe transversale d'une feuille on remarque contre l'épiderme une lame de tissu vert dont les éléments sont perpendiculaires à la surface épidermique. Les cellules forment deux 
assises sur une assez grande étendue; mais celles-ci ne sont pas continues, elles sont interrompues de distance en distance par une grande cellule cylindrique aussi longue que l'épaisseur de la lame. Les grandes cellules sont environ trois fois plus longues rque larges, les petites ont une longueur qui ne dépasse pas d'une fois la largeur.

Au-dessous du tissu en palissade sont des cellules irrégulières et dirigées dans tous les sens

II. Ner $T^{2} u^{2}$ C - L'épiderme présente des poils et des glandes semblables à ceux qui existent sur la lame. Quant aux autres tissus de la nervure, ils offrent les mêmes caractères que ceux de la plupart des Labiées.

Gléchome lierre terrestre. - Glechoma hederacca. I.

I. Lame internenrale. a. Evidène. - L'épiderme forme sur les deux faces une assise de cellules tabulaires dont le contour" est très légèrement sinueux. Il n'y a de stomates qu'it la face inférieure Ceux-ci sont sur des cloisons qui séparent deux éléments de l'épiderme.

L'épiderme supérieur et l'épiderme inférieur produisent des poils et des glandes.

Les poils coniques sont composćs d'une série de quatre à dix cellules, dont les parois sont lisses ou munies extérieurement de faibles tubercules. Ils sont s'xpportés par une proéminence multicellulaire, conique (pl. II, fig. I). Parini les glandes nées sur l'épiderme, les unes, petites et ellipsoïdales. sonc unicellalaires ou bicellulaires; les autres, plus volumineuses, sont furmées de huit cellules placées sur un plan horizontal.

b. Mćsophyllé. - Au-dessous de l'épiderme supérieur sont deux couches de cellules cylindriques vertes dont les superficielles sont deux ou trois fois plus longues que larges. Ce tissu est en contact à sa partie inférieure avec deux ou trois rangées parallèles de cellules qui, sur une coupe transversale, sont allongées parallèlement ì la surface de la feuille. De face elles sont rameuses et laissent entre elles des espaces polygonaux.

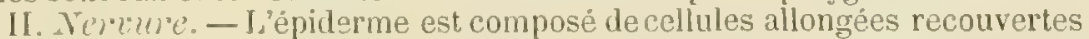
it leur surface d'une cuticule crétée, ct présente des poils et des glandes semblables à ceux de la lame. I es autres tissus de la nervure ressemblent à ceux des Ialuiées.

Origan dictame. - Origanum dictammus. I.

1. Lam intenneurale. a. Epiderne. - Les deux épidermes consistent en une couche de cellules tabulaires dont les parois minces présentent des sinuosités très prononcées. L'épiderme irférieur' produit scul des stomates. Ceux-ci sont elliptiques. Chacun de ses appareils est placé perpendiculairement au milieu d'une cloison qui sépare deux cellules épidermiques.

I es deux ćpidermes sont rerouverts de poils ordinaires et de glandes.

L,es poils, trés allongés, sont composés d'une série d'articles superposés qui donnent naissance à des branches latérales. Ces dernières, assez espacées les unes des autres, sont alternes. La membrane cellulaire de ces appendices est assez mince et lisse.

Les glandes sont de deux espèces. Les unes, petites, unicellulaires et en massue, sont soutenues par un pédicelle généralement deux fois plus long̣ qu'elles et constitué par une file de deux à quatre cellules cylindriques au 
moins aussi hautes que larges. Les autres, presque sessiles et volumineuses, sont composées d'une assise de huit cellules sofparós prar dez cloisons verticales. lilles ne sierrent qu'a la face inferigure rle lib fruille. tandis que les premieres se rencontrent aux deux faces.

b. Misophlulle. - Au-dessous de l'épiderne supvirieur existe uns: couche de cellules cylindrípues vertes deux a trois fois plus longues yue larges. Ce tissu s'appuie inférieurement contre une lame ordirairement plus épaisse et composée de quatre à cinq couches frarallíles de cellules qui, sue une coupe transversale, sont allongées palallelernent à l'épilerme.

II. Téremé - L'épiderme poilu el grlanduleux est formé de cel ules qui, de face, sont rectangulaires et allongres suirant l'axe de la nervure. Les deux épidermes limitent une masse cellulaire dans lápuche plonge un arc libéro-ligneux qui présente les caractèes générax des isutres I aljiés.

Marrube commun. - Sarmebium rulgare. I.

1. Lame intemenale. a. Eniderine. - L'épiderme est revètu de poils et de glandes. Ses cellules, tabulaires. présentent un contour tris sinueux. Les stomates qui n'existent qu'à la face inférieure sont elliptiques. (Jue]ques-uns sont situés entre deux cellules épidermiques, les autres. [ulus nombreux, sont entourés de trois à quatre éléments de l'épiderme

Les poils sont longs, composés d'une rangée verticale de cing à huit cellules plusieurs fois plus liautes que larges. Leur paroi cst mince et lisze. Certains poils s'insèrent ensemble au nombre de deux, trois ou quatre sur une éminence épidermique multicellulaire qui leur est commune. Lorsqu'ils sont ainsi agglomérés. les poils sont rapprochés par leur base et divergent à partir de colle-ci.

Les glandes offrent plusieurs variétés Les unes sont unicellulaires, ont la forme de massue et surmontent un pédicelle crlindriçue unicellulaire en général aussi large que haut. D’autres sont composées de quatre cellules disposées en croix. Les autres enfin rolumineuses. et presque sessiles. sont constituées par une rangẻe horizontale de huit cellules.

b. Mésophylle. - Contre l'épiderme supérieur siére une assise de grandes cellules remplies de chloroplyylle et ayant lisprect de cylindres perpendiculaires i la surface de la feuille, dont la lauteur est trois i cinq fois plus considérable que le diamètre. Au-lessous de cette cou. che en palissade se trouvent trois assises parallèles de cellules. qui. sur une coupe transtersale, sont circulaires ou clliptiques à grand diamètre perpendiculaire ¿̀ l'upiderme. Ces dernières ont leur longuen moitín moindre que les cellules du tissu muritolme.

In n'y a pas de glandes mi de cellules cristalligènes dins le mésophylle.

II. Trereru"e. - L’épiderme est formé d"une couche te cellules allongées dans le sens de la nervure. Il produit des poils et des rylandes semblables à ceux qui existent sur la lame, seulement on rencontre en outre une troisieme espèce de poils glanduleux. I fir glande unicellulaire a la forme d'une calotte sphérique. supportée par un poil bie allulaire. La cellule inférieure de ce dennier est très longue. conique à sommet arondi; la supérieure, petite, moins laroge que l'extrémité du poil. est cylindrique; sa hauteur ne depasse jamais denx tois son diametre. Ces poils glanduleus n'existent qu't la face infélieure de la nervure ou ils sont assez rales.

Quant au tissu fondamental et au système libéro-ligneux ils ofỉrent 
les mêmes éléments et les mêmes dipositions que seux de la plupart des Labiées. On rencontre en effet une ì deux couches de collenchyme, une masse parenchymeuteuse, un arc libéro-ligneux dont le liber possède des éléments à membranes cellulosiques.

Germandrée aquatique. Teucrium scortium. L

I. Lame internemale. a. Enderme. - Les cellules de l'épiderme ont un contour très sinueux. Il existe des stomates, des poils et des glandes aux deux faces de lil feuille. Les stomates sont elliptiques; les uns sont situés cntre deux cellules; les autres, plus nombreux, sont en contact avec trois ou plusieurs cellules épidermiques.

Les poils sont composés d'une file de trois à six articles très longs; ils sont coniques et présentent des parois hérissées de fins tubercules.

Les glandes sont soutenues par un pédicelle unicellulaire ou pluricellullaire. Les unes, quadricellulaires, surmontent un poil cylindrique court. Los autres, sphériques, unicellulailes, sont supportées par un pédicelle formé d'une rangée rerticale de trois à six cellules dont les inférienres sont trois ì huit fois plus longues que larges.

b. Mésomhylle. - Le mésophylle est constitué par trois on quatre assises parallèles de cellules cylindriques ou elliptiques perpendiculares ¿ la surface de la feuille. Tantót les cellules des trois couches sont égales en longueur, tantôt leur hauteur diminue de l'assise supérieure ì l'assise infériemre.

II. Nervure. - Lépiderme, recourert de glandes et de poils semblables à ceux de la lame, est composé d'une couche de cellules allongées. suivant l'ase de la nel'vure, et pourvues de membranes ponctuées. Entre les deux fpidermes se trouve du parenchyme, dont les cellules polyédriques, assez grandes, laissent entre elles des méats. C'est dans ce tissu que siège un cordon libéro-ligneux, en forme d'arc. Le liber est constitué par des éléments dont les parois présentent les rẻactions de la celluluse.

\section{Germandréc petit chêne. Teucrium chamcedrys. L.}

I. Lame intemenrale, a. Epiderme. - Une couche de cellules à contour sinueux. Des stomates à la face inférleure seulement. Ils présentent los mèmes caractères que ceux de la Germandrée aquatique.

Lis mils sont composés de deux cellules superposées. Ils sont tantòt droits, tantôt la cellule supérienre forme un conde avec l'inférieure. La membrane de la cellule supérieure est garnie extérieurement de tuber. cules arrondis.

Les glandes surmontent toujour's un pédicelle unicellulaire très court. Les unes sont unicellulaires sphériques, d'autres bicellulaires, les autres enfin quadricellulaires.

b. Mésophylle. - Le parenchyme en palissade constitue une ou deux couches de cellules dont les s’perficielles sont trois ì cinq fois plus longues que larges. A la partie inférieure de ces assises existe une lame de cellules irrégulières dirigées en tous sens. 


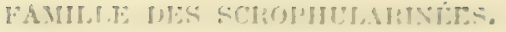

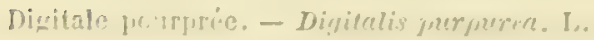

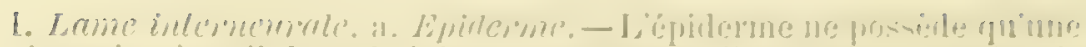

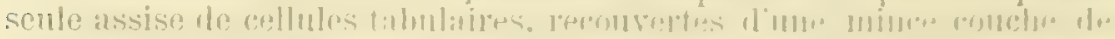

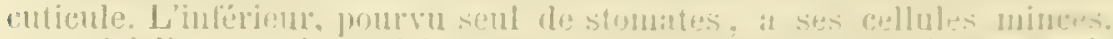

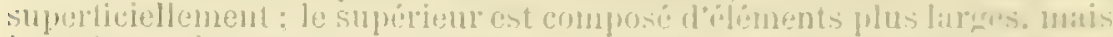

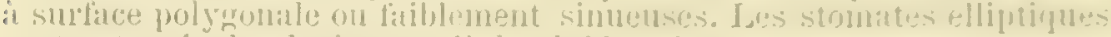
sont entourés de plusicur's cellules épirlerminnes.

Ces deux épidermes sont pourvus de poils orlintires et de proils glanduleux. Les poils proprement dits, coniques. sont constimes jour me

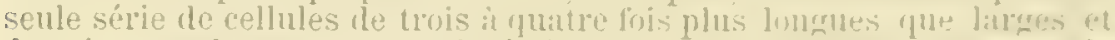
dont les membranes un pen épaissies sont ménéralenent pourvies de petits tubercules extéricurs anondis flul. I. fir. IV.co).

Les poils glanduleux sont beaucoup plus courts; ils sont cylindrinues et le plus souvent lormés de deux cellules superposés. Ils supportent une glande presque splyérique, tantit unicellulaire, tantit bicullulatre (d) ; et dans ce cas, Jes denx cellules sont toujours separees par une cloison verticale.

b. Mésophylle. - Le mésophylle pourva de chnmophylle ne plévente point de cellules cristallirenes, et difrère sur la fice infericure et supé rieure. A la face supérieure, il forme une conche de mrosses cellules cylindriques, courtes, dont la longuenr ne dép:tsse guére trois fois la lanrreur. Ces éléments, pressés les uns contre les autres. sont allonús perpendiculairement ì la surface de lat feuille. Le mésophrlle inférieur. égal au précédent ou un peu plus épais que lui, est composé de trois a quatre couches de cellules cylindrigues; sur une coupe transversale, elles sone rectangulaires, allongées parallilement ì l’épiderme et semblent ne laisser entre elles que de petits méats. Elles présentent. de plus, de finibles prolongements.

II. Marge. - La marge est uniquement constituée par du parenchyme vert coiliẻ de l'épiderme marorinal.

III. Nervure. - La nervure est bi-convere. Elle est limitée en laut et en bas par' de l'épiderme it cellules allongées dians le sens longitudinal. et pourvu contre la lame de poils ordinaires et glanduleur. En dedans de l'épiderme siège une inasse considérable de cellules dans laquelle se trourent plongés un ou trois arcs médullo-libéro-lignevis, tous convexes inférieurement, concar'es supérieurement. Ces cellules, dont le diamètre augmente de l'extérieur il l'intérieur. sont incolores on renferment quelques grains de chlorophylle. Quelques-unes, les plus rapprochées du corton central, contiennent des grains d'amidon. sur une coupe transversale, elles sont polyronales ordinairement à six cutés et litissent entre elles de petits meats triangulaires; sur une coupe lon ritirlunale, elles forment plusieurs tiles de cellules coni pues sépalrées entre clles par des cloisons horizontales.

Liare central est constitué, en allant de bas en hat : $1^{\circ}$ de liber, 20 de bois, 30 de moelle.

Le liber forme à sa partie extérieure un arc continu assez épais de fibres à section irrégulière, polygonale, un peu éfaissies, surtout aux angles, cellulosiques et ì cavité centrale large. E: dedans des fibres 
existe un amas moins développé de liber celluleux dont les éléments sont beaup plus petits.

Le bois, en général de mème puissance que le libel, est constitué par plusieurs lames radiales très rapprochées d'éléments fibreux et vasculaires. I.es fibres sont épaisses, yuadrangulaires, ponctuées; les vaisseaux sont spiraux et trachéens.

La moelle occupe la concavité de l'ar'c, ses éléments sont polygonaux en coupe trunsversale, rectangulaires, cellulosiques, allongés dans le sens de la nervure sur une coupe longitudinale. Ils ont une membrane ponetuéc, épaissie surtout aux angles (cette moelle est donc collenchymatuide).

Digitale à grandes lleurs. - Digitalis grandiflora. All.

La Digitale à grande fleur se distingue de la précédente par la présence de stomates à la face supérieure et par son épiderme peu poilu.

Falsifications. - La Digitale pourprée peut ètre falsifiée par les espèces suivantes: Molènes (Verbascum), Consolide (Symullutım officinale. L.) et Inule (Inula).

Les Trobascrm se distinguent par leurs poils ramifiés (pl. II, fig. III). Le Symphlutum possède de nombreux poils en hamecon et ses glandes externes toujours unicellulaires, arrondies, sont toutes supportées par un pédicelle formé d'une cellule (pl. II, figg. VII). Quant aux muele, on les reconnait à leur épiderme dont les poils ont leurs cellules supérieures, renflées a leur base (pl. I, fig. I), et dont les glandes presque sessiles sont composées de plusieurs rangées horizontales bicellulaires (pl. II, fig. IV). Les glandes, à base elliptique, sont ovoïues.

FA MT?.TE IJES VERBASCEES.

Molène commune. - Verbascum thapsus. L.

Lame interneurale. a. Enillène. - L'épiderme supérieur et l'éplderme inférieur. tous deux pourvus de stomates, forment une seule assise de cellules tres aplaties, minces, sinueuses superficiellement. Les deux épidermes produisent des poils ordinaires et des poils glanduleux.

Les poils sont étoilés, articulés. Les celiules, juxtaposées en série verticales, produisent des branches latéra!es, coniques, unicellulaires. Certains articles ne portent qu'une branche, d'autres donnent naissance à plusieurs disposíes en verticilles. Dans le Teruascum thapsus, les poils sont longs. l'resifue toutes les cellules articulées portent des branches, et lorsqu'il y a un verticille celui-ci est composé de six ì sept rameaux (pl. II, fig. III). Les poils glanduleux courts sont formés d'une ou deux cellules cylindriques plus hautes que larges, placées sur un seul rang. Ils supportent des glandes arrondies, unicellulaires ou bicellulaires it cloison verticale.

b. Mésophylle. - Le mésophylle vert est formé de cellules très pres. sées les unes contre les autres. I la lace supérieure, il constitue deux couches de petites cellules cylindriques, deux fois plus longues que larges. Au-dessous existent trois à cincl assises de cellules arrondies ou ovales, ou rameuses, dirigees en tous sens.

II. Marge. - La marge est constituée par la terminaison latérale de l'épiderme et du parenchyme. 
III. Tenvure, - I a nervure est bi-convexe. Fin dedans des deux éridermes glanduleux et poilus se trouvent des cellules un peu cullenchiviateuses sous forme de prismes allongés it base polfegonale, presigure récruliêre et à parois épaissi 's surtout aux angles. Cé callenchyone est un peu plus développé à la face inérieure. Entre les denx arn:s se troure un massif cellulaire puissant dans lequel siege un cordon libéro-Jizneux. Il est constitué par des cellules it membrane mince qui laissent entre elles de petits méats, ont un contonr polygonal sur une coupe transversale, ct une surface rectangulaire dans le sens longiludinal. Ces éléments sont incolore: et les plus voisins du cordon vasulaire contienment quehiues frains d'amidon.

Le cordon central a la forme d'un arc, convexe inférieurement, coneave supérieurement et dont les extrẻmités supérieures se recourbent en lass vers le centre. Il est constitué par du liber et du bois.s.

Le liber est fibreux extéricurement. Les fibres longues it membranes cellulosiques, un peu épaissies, principalement vers les angles. el à cavité grande, se groupent pour former des ilots séparés par des cellules semblables it celles qui existent en dehors de lui. Au-dessus de ces fibres vient le liber celluleux dont les élẻments minces, polyconaux. sont dispusés en séries radiales.

Le bois, égal au liber. est constitué par des bandes vasculaires séparées les unes des autres par des espèces de rayons médullaires à un ou deux rangs de cellules minces.

Contre la face supérieure du bois est adossé un umas deélèments cellulaires, qui, régulièrement polygonaux en section transtersale, rectanculaires, quatre à cing fois plus longs que larges, en section longiludinale, ont leurs membranes épaissies aux angles, comme un collenchyme.

Molene noire - Verbascum nigrum. L.

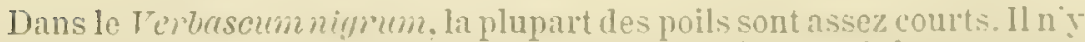
a d'ordinaire que la cellule terminale qui donne missance à des rameatus disposés en rerticille.

Ces espèces de Feruascum peurent ètre substitués it lia Dirritale (Digitalis purpurea. L.).

F.IIILLE DES SOLAXEES.

Espices: Morelle norre. Solamun niquam. - Datura stramoine. Dalura stremonim. - Belladone. Atropa belledona L. - Nicotiane taluac. Vicotiuna tabarems. I.. Xic. rustique. $N_{\text {. }}$ renstica. L. - Jusquiame blanche. Hyuscyganus albues. L. - Jusquiame noire. Inyoscyamus niger. L.

1. Lame interneurale. a. Eniderme. - Lépiderme, toujours pourvu de stomates sur les deux faces, est compose dune couche de cellules dont les parois sont sinueuses. Il donne naissance a des poils ordinaires et it des poils glanduleux.

Les poils sont torjours pluricellulaires et leurs éliments sont super. posés de manière ì former une file rerticale de cellul:s. Leur membrane est tantôt lisse (Nicotiana. Hyoscyames), tantòt tubsrculeuse (Dutuin stramonizm, Aliopa bellatona).

Les glandes sont tantôt unicellulaires, tantôl pluricellulaires. Dans 
- Itropa belladona. L., IIyoscyamus, Nicatiana tabacum. L., on trouve plusieurs sorte de glandes: les unes sont composées d'une cellule, les antres de plusieurs cellules disposées sur plusieurs rangées horizontales. Dans la Solanum nigmun. L. et le Datura stramonium, elles sont toujours pluricellulaires et leurs éléments sont superposés en rangées horizontales ordinatiement formées de plusiem's cellules. Quant ả leur forme, elle est variable. En Dahure (pl. III, lig. III), elles ont l'aspect d'un cône tronqué ì grande base superieure. Certaines glandes d'Atropa belladona. L. (pl. II, fig. VIII) sont ovolides ì grosse extrémité supérieure. Les glandes de IIyoscyamus et de Nicotiana tabacum. L. Sont oblongues ou oroïdes (pl. III, fig. VLI).

Le pédidelle qui soutient les glandes est tantòt unicellulaire (Solamm nigmum, Datura stiammomu) (pl. III, ficg. VII) et quelques poils glanduleux d'Atropra belladona. L. (pl. V. fig. VI, a), tantôt pluricellulaires unisériés en vicotiana, Hyoscyamus (pI. III, fig. VIII) et Atropa belladome (pI. II, fig. VTlI).

b. Mésophylle. Le inésophylle est hétérogène asymétrique en Alropa belladona. L., Nicotiana, Dalura, Solamum.

Le parenchyme en palíssade est composé d'une seule couche. Dans les Hyoscyamis il existe sur les deux faces une assise de cellules allongées. dirigées perpendiculairement à la face de l'épiderme. Le mésophylle renferme des cellules cristallirènes. En Atropa belladona, chaque cellule contient une quantité innombrable de cristaux très petits icristaux pulvérulents). Le Datura et l'IIyoscyamus albus. L. possèdent de cros cristaux agglomérés. tandis que dans la Hyoscyamus niger. L. ils sont presque tous isolés. Enfin le parenchyme vert de Solanm nigrma est dépourvu de cristaux.

II. Marge. - Le bord foliaire est tonjours constitué par du parenchyme vert recourert d'épiderme.

iII. Tervure. - Contre l'épiderme supérieur et l'épiderme inférieur' stomatiques, poilus et glinduleux, s'appuient quelques couches de collenchyme ordinaire convexe. Entre le collenchyme supérieur et le collenchyme inférieur siège un amas de grandes cellules incolores contenant quelques grains de chlorophylle. Quelques éléments de ce tissu sont remplis de nombreux cristaux pulvérulents. C'est dans l'intériem de ce parenchyme qu'est plongé un arc libéro-ligneux assez étendu et composé, en allant de bas vers le haut, de liber inferrieur, de bois, de liber supérieur (pl. VI, fig. II, h. h, d).

Le liber inférieur et le lili... supérieur sont constitués par des îlots de fibres et de cellules dont les parois sont toujour's cellulosiques. Le bois est formé d'éléments vasculaires.

Caractères commus: Stomates aux deux faces. Poils ordinaires et poils glanduleux. Point de glandes internes. Liber interne en illots. Des cristaux pulvérulents d'oxalate de chaux.

\section{Belladone officinale. - Atropa belladona. L.}

1. Lame interneurale. a. Enileranc. - L'épiderme supérieur et l'épiderme inféricur, munis de stomates clliptiques, forment une seule couche de cellules tabulaires à parois sinueuses. L'épiderme est tantôt glabre, tantôt pourvu de poils composés d'une seule rangée de trois à cinq cellules cylindriques allongées suivant la longueur du poil et dont les membranes épaisses sont tuberculeuses extérieurement. 
on rencontre anssi it lit fucc inferieure des poils surnontes disne flande.

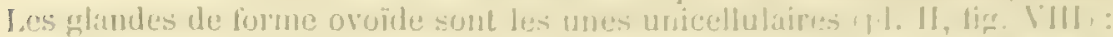

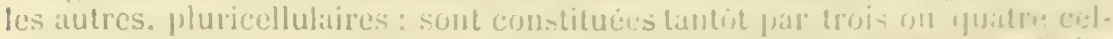

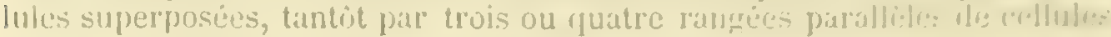
(p). V, lig. VI, al).

Le pédicelle est unicellulaire (pl. V, fig. VI, a) ou cat composis llune file de deux it cinc cellules cylindriques (pl. II, ligr. VIII)

b. Mésumhlle. - Le mésophylle est liétéromene. Le parenchyme -upérieur posside une seule couche de cellules cylindriques trois al ifuatre foiplus longues que larges et perpendiculaires a la surface foliarie rpl. l, lig. VI, a).

Le parenchyne inférieur, égal au supérieur ou souvent moins développé que ce dernier, a au contraire ses callules rameuses, laissant entre clles des méats irréguliers. Cie tissu est pursemé, prés du parenchyne supirjeur, lis. cellules plus grosses, arrondies. contenant des cristaux nombreux et tré petits d'oxalate de chaux (cristaux pulverulents (pl. l, liz. . $1 \%$. d).

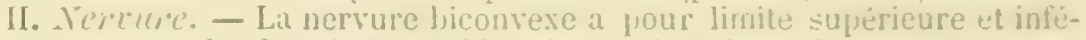
rieure une couche de cellules épidermiques dont la surface est lectanfrulaire ou polygonale à côtes irréguliers. Ces ce!lules sont allongcies suivant la nervure. Les deux épidermes sont munis de poils ordinaires et frlanduleux plus nombreux que ceux qui existent sur la lan'e et ressemblant it ces derniers. Entre les deux épidernes existent du collenchrme, du tissu collenchrmateux et un cordon libero-ligneux. Le collenchyme forme is la face supérieure quatre à cing couchus de cellules cylindriques ou pristra. tiques à base légèrement polygonale. Les élénents supérieurs punctués sont généralement dépourvus d'épansissements aux angles; il n'y a que les inférieurs qui présentert deś épaississements, dont quelques-uns font saillie dans la carite cellulaire.

A la face inférieure il existe trois i quatre couches de semblables cellules mais plus épaisses. Les cellules inférieures laissent entre elles de grunds méats losangiques et les supérieures offrent seulement aux angles des épaississements qui s'avancent très peu dans la ravité cellulaire. Le collencliyme n'a donc son réritable caractère (que dans les cellules les plus rapprochées du centre de la nervure. et. de plus. il est un peu convere. Le lissu parenchymateux placé entre ces deux collenchymes possède de grandes cellules polygonales en section transversale. rectangulaires en section longitudinale. Ces cellules à parois assez minces, ponctuées, laissent entre elles de petits méats. Elles sont incolores, car le parenchỵme vert de la lame ne s'étend point dans la nervure. Quelques-uries renferment de nombreux cristaux pulvérulents.

C'est au milieu de cette masse qu'est plongé un cordon libéro-ligneux qui a la forme d'un arc concave supérieurement. Il est constitué en allant

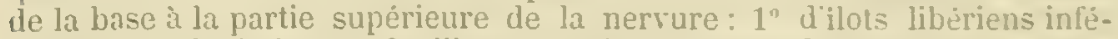
ricurs; : 20 de bois; 30 de liber supérieur séparé du bois par quelques couches de cellules. Le Jiber externe (fl. VI. fig. T, b) est disposé en iluts séparés les uns des autres par une file de une it trois cellules allongées de haut en bas (a). Chaque ilót est composé inférieurement de quelques fibres cellulosiques à menbrane un peu épaissie surtout aux angles, et supérieurement de petites cellules grillađẻes ou sẻreuses. Le bois est formé de plusieurs bandes ravonnantes de vaisseaux ponctués, rarés, spiraux, spiro-annelés et trachées. Ces bandes sont séparées entre elles par une rangée au plus de cellules. Les rayons parenchỵmateux se continuent en 
bas arec les rangées intro-libériennes, et se confondent en haut avec du parenchyme dont les éléments, très allongés suivant la nervure, sont prismatiques à base polygonale et sont pourvus de membranes ponctuées, épaissies surtout aux angles. Quelques-uns d'entre eux contiennent de très pelits cristaux d'oxalate de chaux.

Le liber supérieur forme plusieurs ilots analogues ì ceux du liber inféricur (h).

Feuille de Iatura stramoine. - Datura stramonium. L.

I. Lame interneurale. a. Epides'me. - L'épiderme supérieur et l'épiderme inférieur sont composés d'une seule couche de cellules tabulaires, it parois minces, un peu ondulées superficiellement, rectangulaires en coupe transversale. On reneuntre sur ces deux épidermes des stomates elliptiques disposés irrégulièrement, des poils simples assez rares et des poils glanduleux. Les poils coniques sont constitués par une seule file de trois it cinq cellules assez grandes dont les parois épaissies sont garries de petits tubercules arrondis.

Les glandes sont supportées par un pédicelle unicellulaire courbe et dont la cellule cylindrique est de une à quatre fois plus haute que large. Cies glandes (pl. III, fig. VII) ont ginéralement la forme d'un còne tronqué dont la grande base opposée ì l'extrémité du pédicelle est convexe. Elles sont composées de deux rançées superposées et parallèles de cellules; l'inférieure a deux ou quatre cellules, la supérieure de quatre à huit callules allongées parallelement au poil.

b. Mésophylle. - Le mésophylle est constitué par une masse de cellules vertes différentes sur la face inféricure et sur la supérieure. Le parenchy me supérieur a une couche de grandes cellules cylindriques en palissade, souvent minces. Le parenchyme infërieur, presque aussi puissant que le supérieur, a ses cellules rameuses allongrées parallèlement à l'épiderme. On rencontré dans ce parenchyme, tout contre les cellules en jalissade, de nombreuses cellules isolées renfermant des cristaux agrglomérés d'oxalate de chaux.

II. Mraryc. - La marge est constituée par la terminaison latérale du parenchyme chlorophyllien.

III. Tereure. - La nervure bi-convexe présente à sa surface des cellules épidermiques très longues, qui, vues de face, ont les parois longitudinales droites et parallèles, tandis que les transversales sont tantot per. pendiculaires, tantôt obliques aux premières. L'épiderme, dépourvu de stomates, est muni de gland:- ut de poils semblables à ceux qui se trouvent sur l'épiderme de la lame. Plusieurs assises de collenchyme sont adossées à des membranes épidermiques; ce collenchyme est en général plus puissant sur la face supérieure. Au nombre de quatre à six couches à la face supérieure, de trois à quatre couches à l'inférieure, les cellules ont la forme de longs prismes à base pólygonale. Leurs parois sont minces, si ce n'est aux ingles où elles présentent des épaississements convexes faisant saillie dans la carité cellulaire. Entre les deux collenchymes convexes existe un amas cellulaire dans lequel existe un arc libéro-ligneux. Les éléments minces qui le constituent sont prismatiques à base polygonale et laissent entre eux de petits méats. Quelques-unes de ces cellules sont remplies de cristaux, les uns pulvérulents, les autres tétraédriques, d'oxalate de chaux.

Quant au tissu vert il n'occupe que les parties latérales extrèmes de la nervure. Le cordon libéro-ligneux, très étendu, a la forme d'un arc concave 
supérieurement. Il est composé, en allart de has en haut: fo de librer

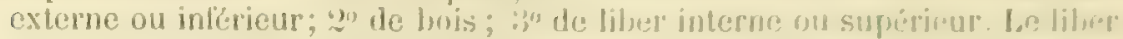
externe est formo de plusieurs arounes tanrentiels sifario las uns des

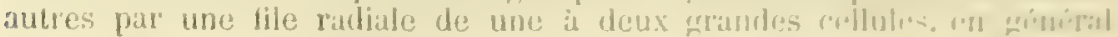
allongées radiatement et renfermant de la chlorophifle. Charge wroupe ast constitué par de larges cellules grrillagrées et séveuses it monblanes un peu épaissies, très réfringentes.

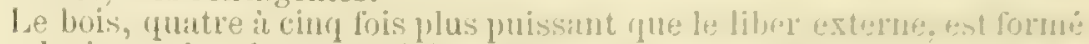

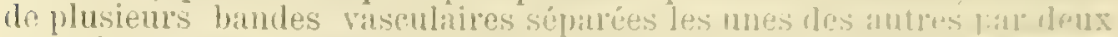
ou trois rangées de plusieur's grandes rellules at chlorophylle. allongres

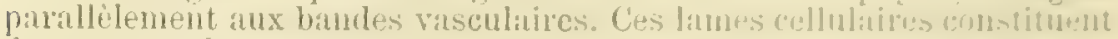
des rayons. Quant aux bandes vasculailes, elles sont rounpreses de une it trois files de gros valisseatux ralyés, ponctués, spiraux, cannelé et irachées.

Le liber interne, séparé du bois par une ou deux conches de cullules minces, grandes, prismatiques, contenant de lat chloruphylle (cellules médullaires), a la mème constitution et la méme puissance que le liber externe.

\section{Feuille de Morclle noire. -- Solanum nigrum. I..}

1. Lame internerrale. a. Eviderne. - L’épiderme supirieur et lëpiderme inférieur sont constitués par une seule couche collulate. Les éléments, moins larges sur l'épiderme inféricur, sont tahulaires. très sinteux, vus de face. Des stomates, des poils simples et rlandulenx se remarquent sur ces deux épidermes. Les poils coniques sont à une seule rangrée de trois ì cing cellules plus longues que litrires et dont les membranes épaissies présentent extérieurement des tubercules arondis. Les stomates, elliptiques, disposés sans ordre. sont presque tous placis contre une partie de la paroi interne de cellules épidermiques. Les grandes, rares sur la lame, sont soutenues par un pédicelle court, deux it trois fois plus long que lirge. En forme de cone tronuué, elles sont cumposées de deux assises perpendiculaires ir la direction des poils. Liassise inférieure consiste en une, deux, quatre ou six cellules; li supérieure en deux, six ou huit cellules allongées suivant la longueur des poils.

b. Mésophylle. - Le mésophylle hétérogène ne renferme point de cellules a cristaux. Le parenchyme supérieur est en palissade. Les cellules cylindriques sont quatre fois plus longues que larges. Le parenchyme inférieur, un peu moins déreloppé que le supérieur. miésente trois à quatre couches de cellules rameuses, généralement étenclues parallẻlement à la surface foliaire (en coupe transversale) et présentant de face des branches courtes disposées très irrégulièrement.

II. Marge.-L'extrémité latérale du parenchyme vert et de l'épilerme constitue la marge.

III. Nerence. - La nervure est trés convese. Son épiderme supérieur et son épiderme inférieur sont à une seule couche de cellules trẻs allongées en forme de prisme à base carlée ou rectangulaire, tantòt perpendiculaire, tantôt oblique aux arètes. Ces deux épidermes sans stomates sont munis de poils et de rlandes plus communs que sur la lame. Contre ces deux épidermes, existe du collenchyme convere plus puissant it la fince supérieure. Il forme deux couches inférieurement, trois it quatre couches supérieurement. Les cellules ont l'apparence d'un prisme droit 
allonge à base polygonale (quatre à six côtés), et hà hateur cinq à quinze fois plus grande que la grande diagonale de base.

Entre le collenchyme se trouve le tissu fondamental dans lequel siège un arc libéro-ligneux. Ce tissu fondamental est constitué par un amas de cellules grandes a membranes minces et laissant entre elles de légers méals. Ces éléments prismatiques, à base polygonale, sont incolores. Il n'y existe point de parenchyme vert.

L'arc libéro-Jigneux est concave supérieurement, convexe inférieurement. Du liber externe, du bois et du liber interne, avec des rayons médullaires, entrent dans sa constitution. Le liber externe forme de petits ilôts séparés les uns des autres par une ou deux cellules prismatiques ì membrane mince. Chaque jlôt renferme, inférieurement, quelques fibres cellulosiques, nacrées, à section polyg,gonale et épaissie aux angles; supérieurement, un petit massif de cellules grillagées et sévcuses épaissies.

Le bois, deux à trois fois plus puissant que le liber externe, forme plusieurs bandes vasculaires composées chacune d'une à cinq files de vaisseaux rayés, potctués, spiraux, spiro-annelés, trachées. Ces bandes sont séparées les unes des autres par une file de cellules prismatiques ́rayons médullaires), à base rectangulaire allongée parallèlement aux bandes. Le liber interne, de puissance égale à celle du liber externe, est constitué de la même manière. Il ne touche pas le bois, mais en est séparé par des cellules médullaires à membranes minces plus petites que les cellules du tissu fondamental proprement dit.

Jusquiame noire. - H1yoscyamns niger. L.

I. Lame interneurale. a. Epideme. - Une seule couche de cellules constitue l'épiderme supérieur. Les cellules sont irrẻgulières, à contour sinueux superliciollement et à membrane mince. Des stomates s'y rencontrent ainsi que des poils ordinaires et des poils glanduleux (pl. III, fig. VIII). Les poils, longs, sont constitués par une série de grandes cellules, quatre à dix fois plus longues que larges et dont la largeur diminue insensiblement de la base vers le sommet. Leurs membranes peu épaissies sont dépour.. vues de tubercules. Les poils glanduleux longs, toujours constitués par une seule série de cellules allongées suivant la longueur du poil, supportent une glande généralement en massuc. Cette glande est unicellulaire ou pluricellulaire. Lorsqu'elle est pluricellulaire, elle est forınée de deux ou cinq cellules parallèles séparées par des cloisons perpendiculaires au poil ; ou bien de deux ou quatre rangs parallèles de celluies dirigées parallèlement au poil.

b. Mésopllylle. - Le mésophylle vert est hétérogène. Le supérieur est is une seule couche de longues cellules cylindriques, muriformes, quatre it cinq fois longues que larges. L'inférieur a des cellules dont la forme et la direction varient suivant la position. Contre l'épiderme inférieur existe de plus longues cellules de deux à trois fois plus longues que larges, à peine rameuses et laissant entre elles de faibles méats. Dirigées perpendiculairement à la surface épidermique, elles constituent une espèce de parenchyme en palissade.

Viennent au-dessus de cette couche plusicurs langs de cellules iso. diamétriques ou allongées. Dinns la couche supérieure de ce parenchyne se trouvent disséminées des cellules vertes qui contiennent, pour la plupart, un ou deux cristaux isolés d'oxalate de chaux. Quelques-unes cependant renferment des agglomérations cristallines. 


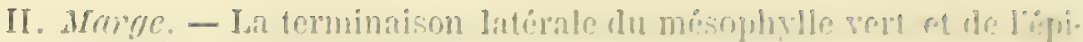
rerme constitue seule la marre.

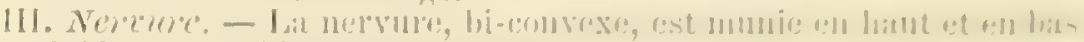

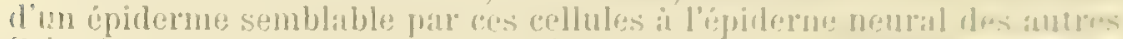
Solanées et pourvu de poils ordinaters et mlandulenx comme il en miste

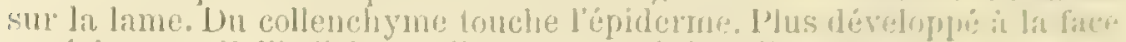

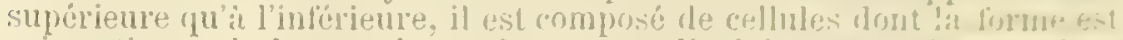
prismatique, it base polygonale, perpendiculates anx aretes et lont les membranes présentent aux angles des épalisissements dont qual-

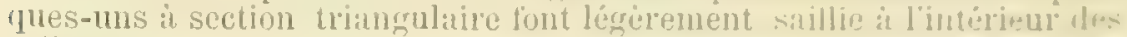
cellules.

Entre ces denx collenchymes sière une masse de mpantes cellules la plupart incolores, phus longues, en forme de prisme droit it base prolyon. nale et à membrane mince. Quelques éléments placés ver's to centre it. la nervure renferment duns leur cavité une yuantite considérable de petits eristaux. Cest dans ce tissu que se trouve placé un arc libéro. ligneux trẻs étendu latéralement et un peu concave. II a lat mème constitution que le cordon que l'on rencontre dans la plupart des. Solnices. Il est composé en ellet de liber externe, liber interne. bois. moelle et rayons médullaires. iLe bois, formé d'un grand nombre de bandes: rayonnantes viscula res, a une puissance igale on un pen supericure it celle du liber.

Jusquiame blanche. - Hyoscyrmus allus. L.

La Jusquiame blanche ne se distingre de lin précéchte que par le contenu des cellules cristalligines que lon rencontre dans le mésophrile. T'andis que dans la Jusquiame noire, la plupart des cellules cristalligènes renferment de gros cristaux isolés. Jinns la insquiame blanche. elles contiennent des cristaux agglomérés assez réruliers (p!. V, lirr. IV, c).

Feuille rle Nicotiane taluac. Tabac, - Vicotinna tabnouir.

1. Lame intemenale. a. Épiderine. - L'épiderme, tant supérieur qu'inférieur, est it une seule couche de cellules at membrane mince ct i contour très sinueux en surface. On y rencontre des stomates. des poils unisériés et des poils glanduleux.

Les poils glanduleux sont de deux sortes: les uns (les plus tonmmuns) sont tres longr, multicellulines; les autres, plus rates, unicellulaires.

Les pluricellulaires sont composés d'une série plus on moins nombreuses de cellules très allongées suivant la longuem du poil ; ils supportent des glandes en massue, tantüt unicellulaires, tantut composées de plusieurs cellules disposées en plusieurs rangs paralliles. Les poils courts sont trés larges, leur longueur dépasse it peine deur fois la largeur. Leur sommet est terminé par une glande en forme de dome placée d'ordinaire obliquement et composée de une it deux rangées sourent multicellulaires (quatre it huit en chaque ramir.)

b. Mésophylle. - Contre l'épiderme supérieur s'ipplique une couche de cellules cylindriques dont la longueur dépasse trois ou quatre fois la lirrgeur. L'inférieur, presque aussi développé que le supérieur, possède deux à quatre langs de cellules rameuses, la plupart parallèles à la surfice foliaire (coupe transversale). On rencontre dans ce parenchyme 
de très rares cellules contenant des cristaux pulvérulents d'oxalate de chanx.

II. Marge. - La marcre est constituée par des cellules vertes provégées latéralement par des cellules épidermiques.

III. Nereure. - La nervure est bi-convexe. Son épiderme, semblable it celui des autres Solanées, présente des poils et des glandes analoguse ¿̀ ceux de la lame. Contre l'épiderme inférieur sont adossées trois à quatre couches de grandes cellules collenchymateuses; il y en a quatre ¿̇ cinq a la face supérieure. Ces cellules, dans l'intérieur dequelles existent parfois quelques rrains de chlorophylle, sont très longues, prismatiques, à base polygonale (quatre à luuit côtós). Elles présentent ả leurs angles de petits épaississements dont la sectiont transverse est triangulaire; un grand nombre de ces derniers savancent un peu dans la cavité cellulaire; ces deux collenchymes enlerment un massif puissant de grandes cellules à parois minces laissant entre elles des mẻats. Ces élérnents reetangulaires en coupe longitudinale, polygonaux sur une coupe transversale. laissent entre eux de petits méats et sont incolores ou renferment de rares grains de chlorophylle. Quelques-uns, centraux, contiennent des cristaux pulvérulents.

Le cordon libéro-ligneux a la forme d'un arc très étendu, il a la même constitution que celui des autres Solnmées. In liber externe, du bois plus développé que ce dernier, du liber interne qui est séparé du bois par des cellules minces, médullaires, composent ainsi cet arc.

\section{Nicotiane rustique. - Nicotiana rustica. L.}

Se distingue du précédcnt par son collenchyme inférieur qui ne possède qu'une assise cellulaire.

\section{WAMILLE DES BORRAGINEES.}

Especes: Bourrache officinale. Borrago officinalis. L. - Consoude officinale. Symphyium officinale. L. - Buglosse officinale. Anchusa officinalis. L. - Pulmonaire officinale. Pulmonaria offechalis. L. - Cynoglosse officinale. Cynoglossum officinale. $\mathrm{L}$.

I. Lame intemeurale. a. Evideme. - L'épiderme forme aux deux faces une couche de cellules tabulaires à contour sinueux (s!mmphly/rma) pl. II, fig. VII), (Anchusa Cynoglossmm) ou polygonal (Pulmonaria, épiderme supérieur de Borrajo). Des stomates se rencontrent aux deux faces, sauf dans la Pulmonaria officinalis. L., qui n’en présente point ì la face supérieure.

L'épiderme est garni de nombreux poils ordinaires et de poils glanduleux. Les poils ordinaires sont tous unicellulaires, la plupart ont leur base enchâssée dans l'épiderme entre plusieurs cellules; d'autres, plus forts et moins nombreux, sont supportés par une proéminence pluricellulaire épidermique. Coniques dans la Bomrago, l'Anchusa, la Pumonaria, la Cymoglossum, ils ont la forme (au moins la plupart) d'hameçons dans le Sympinylum.

Les poils glanduleux sont unicellulaires dans Borrago, Anchrısa, Symphytum (pl. II, fig. III). Pluricellulaires unisériés en Pumonaria officinalis (pl. II, fig. X). Quand les poils sont composés d'une cellule, cette derniere est toujours jus haute que litrge. Les glandes sont arrondies 


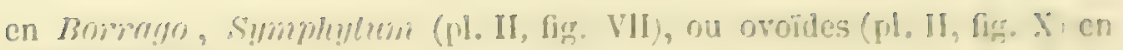

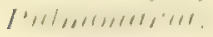

b. Mésomblle. - Ice mésophylle, toujours dipourva de cullules cristalligenes, est hétérorgene isymétrique. loc parenchwe en pali-sarle forme

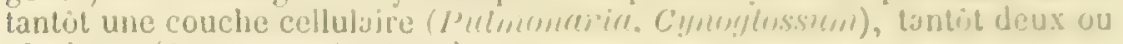

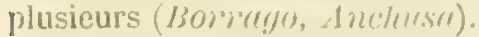

II. Nerture. - La nersure est limitée latéralenent far de lépiderme dont les cellules, allongrées suivant la longueur, sont munies de proils et de glandes. Cet épiderme touche, it la face inférieure et it la face supérieure, quelques couches de collenchyme tantót convese, tontôt coneave; entre le collenchyme inféricur et le collenchyme supérieur est étendu un massif assez puissant de grandes cellulas incolores, à membrane mince, et laissant entre elles des méats. C'rst dans ce tissu parenchymateux qu'est plongé un cordon composé : $1^{\circ}$ is sa fice inférieure d'un arc de liber dont les élíments fibreux al celluleux ont leurs parois cellulo-iyues et forment des amas continus: 20 d'un arc de bois appuyé sur la face inféricure du lilere et constitué par des lames de gros vaiscenux (spiraux, trachées); :,0 d'un tissu cellulaire qui occupe la concavité que forme le ligneux à la partie supérieure. Les cellules qui entrent dans la constitution de ce tissu ont leur membrane épaissie, d'aspect collenchymateux.

Caractires communs: Poils tous unicellulaires. Poils glandu'eux it glandes unicellulaires ou pluricellulaires dont les cellules sont séparées par des cloisons verticales. Point de poils glanduleux en Cynonlossum. Point de cellules cristalligènes. Point de glandes internes. Point de liber interne en ilôts.

Bourrache oflicinale. - Borrago officinalis. L.

1. Lame interncuralè. a. Eniderime, - L'épiderme inférieur est formé d'une seulc couche de cellules tabulaires à membrane très mince, parais. sant plissée superficiellement. L'épiderme supérieur possẻde au contraire des cellules peu sinueuses, irrégulièrement polygonales. Ces deux épidermes sont munis de stomates, de poils ordinaires et de poils glanduleux. Les stomates, irréguliè ement disposés, sont elliptiques et entourés dle plusieurs cellules épidermiques. Les poils coniques ont leurs membranes ćpaissies. Quelques-uns ont leur base soutenue par une proéminence mul. ticellulaire.

Les glandes unicellulaires sont sphériques et supportées par un poil cylindrique deux à trois fois plus long que large.

b. Misolinylle. - Le mésophille vert, hétérogène. ne renferme point de cellules spécialement cristalligènes. Le tissu supérieur en palissade est composé de deur assisns de grandes cellules à membrane mince el allongéps perpendiculairement à la surface foliaire. Ces éléments sont cylindriques et les plus superficiels sont plus longs. Le plus grand diamitte de ces dernier's est double ou triple du plus petit.

Le mésophỵle inférieur, en gẻnéral moins déreloppé que le supérieur, a ses éléments rameux qui, sur une coupc transrersale, semblent rectang̣ulaires, allongés parallèlement ì l'épiderme.

II. Yervere. - La nervure est convexe inférieurement, un peu concave il sa partie supérieure. Son épiderme. dépourru de stonates, is ses cellules allongées suivant la nervure. Il est pourvu de poils à sommet glanduleux, de poils ordinares dont les plus grimds ont leur base en partie emolhissé 
dans une próntinence épidernique multicellulaire Fn dedans dı: l'épideruse viennent deux ou trois couches de collenchyme convexe dont les cellules ont la forme d'un long prisme droit ì base polygonale. Entre le collenchyme supéricur et le collenchyme inférieur siêgent un tissu cellulaire à éléments minces, grands, incolores ou renfermant peu de chlorophylle et laissant entre cux des méats; ils sont prismatiques à base polygonale. C'est dans ce parenchyıne que se trouve placé un arc libéru-ligneux, convexe en bas, concave en haut et dont la concavité est remplie de cellules à aspect collenchymateux.

I.e liber collenchymatoïde possède des fibres et des cellules toutes cellulosicjues et formant un arc continu.

Quant au bois, il est représenté par des vaisseaux qui ne forment pas de bandes radiales bien régulières.

$$
\text { Buglosse otlicinale. - Anchusa oflicinalis. L. }
$$

La Buglosse officinale ( 1 nchusa officinalis. L.) a beaucoup de ressemblance avec la Bourrache Elle se distingue de cette dernière par les cellules de son épiderme supérieur dont le contour est très sinueux.

Cynoglosse ofticinale. - Cynoglossum officinale. L.

I. Lame interneurale. a Epiderme. - Les cellules de l'épiderme sont tabulaires: celles de l'épiderme inférieur plésentent un coutour très sinueux. tandis que celles du supérieur sont polynonales ou légèrement ondulées. Les deux épidermes possèlent des stomates et produisent des poils ordinailes. Ils ne donnent pas naissance a des poils glanduleux.

Les poils ordinaires sont unicellulaires et coniques. Leur base, assez large, est enfoncée dans l'épiderme et se rétrécit brusquement au niveau de la sulface foliaire en se continuant avec le reste du poil qui est assez lin.

b. M'sophylle. - Le mésophylle est dépouivu de cellules cristalligènes. Il formo contro l'épiderme supériem une couche de cellules cylindriques trois ì quatre fois plus longues que larges Les éléments voisins de l'épiderme inférieur sont aussi cylindriques et perpendicu. lailes a la sufuce de la feuille. mais leur longueur ne dépasse pas denx fois leur largeur. Entre ces deux assises s'etendent quelques couches de cellules rameuses qui paraissent elliptiques, circulaires ou rectangulaires lorsqu'on examine une coupe transversale.

II. Tervure. - L'épidelme, poilu. est composé d'une couche de cellules allongées suivant l"are de la nervure. Les deux épidermes sont en contict avec quelques couches (deux it quatie) de grandes cellules collenchymateuses dont les unes présentent aux angles un épaississe. ment quí fait saillie dans l'intérieur des cellules.

Entre le collenchyme supérieur et le collenchyme inférieur siége une masse parenchymateuse considérable dans laquelle sont placés un ou plusieurs corlons. Les éléments qui constiuent ce parenchyme sont volumineux et laissent entre eux des méats. La coupe transversale de la nervure montre leur forme polyonale. Leur yaroi est tres mince.

Chaque cordon est composé de la partie inférieure à la partie supérieure : 


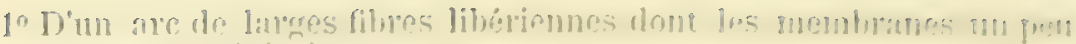
éricsies sont cellulosinues:

- yo l)im aloc do liben" cellulenx:

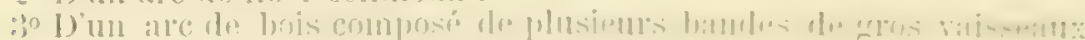

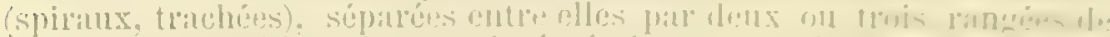

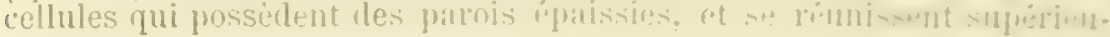
rement:

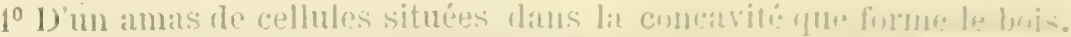

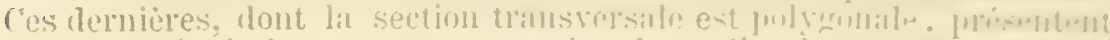

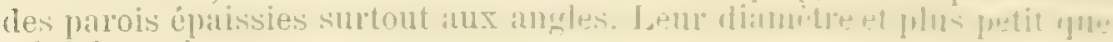
celui des valsseitux.

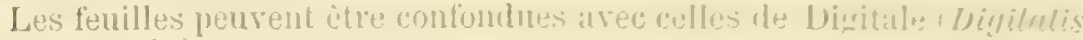
purpurea. L.).

Consoude ofticinale. - symjuntum offirinale. 1.

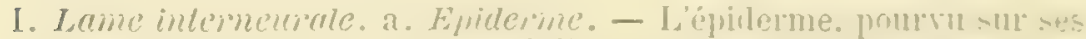
deux liaces de stomates. est composé d'une seule assise de collules talulaires qui, rues de face. présentent une membrane mince tres sinnente. On y rencontre des poils unicellulares de deux sortes: les mns. usser. grands, sont coniques, dronts, et ont une membranc épaisse de maniere it fermer" sourent la carité intérienre. Les antres (11. II. fir. III). plucourts. sont recourbés en hamecon il leur sommet. Outre ces prils. il

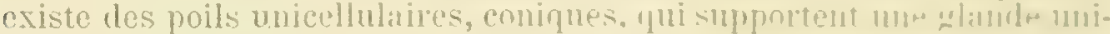
ceellulaire sphérique.

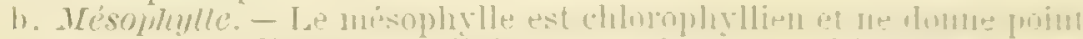

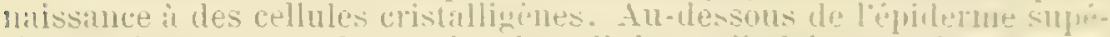

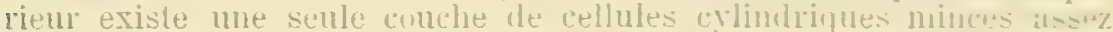
pressées les unes contre les antres et disposées on palis iule. Elles sant généralement deux ì trois fois plus longues que lin pres.

Le mésophrlle inférieur est ménticue et plus épais nut le supérinn. Il est composé de cellules qui sont dordinaire rectantrulaties sur une coupe transversale, il grands cotés paralleles it lis surfice foliatre.

II. Marlle. - L'extrẻmité latérale du parenchyme finliare et ile l'éniderme constitue la rérgion marginale.

III. Norme. - La nerrure. convexe inferienrement est concave supérieurement. Son épiderme. pourvu de poils et de irlamles semblitbles it cenx qui existent sur l'épiderme de la lame. al ses éléments allongés suivant la longueur de la nervure. (ouant aux autres tisins neuran. ils ressemblent il cens des autres Borrarinés.

Pulmonaire otlicinale. - Pulummaria offirinalis. L.

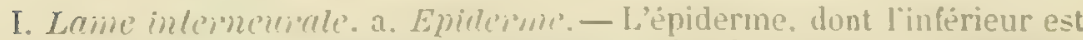
seul muni de stomates, est composé d'une assise de cellules it membrüne mince, tabulaires et polygonales superticiellement. Il donne naissance i des poils ordinaires et it des poils glanduleux.

Les poils ordinaires, coniques, sont de deux sortes : les uns courts, les autres assez longs ont leur base supportée par un mamelon multicellulaire

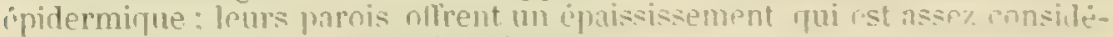

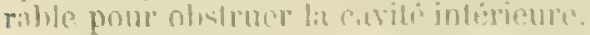

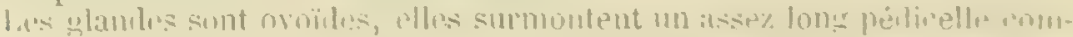

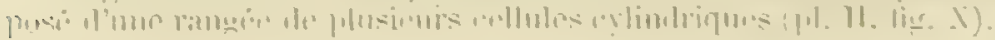


b. Mésophylle. - Le mésophylle supérieur forme une seule couche de cellules en palissade, cylindriques, minces, de deux a trois fois plus longues que larges. Le mésophylle inférieur, généralement plus épais que le supérieur, a des cellules rameuses laissant entre elles de nombreux méats. Sur une coupe transversale, elles paraissent rectangulaires à grand côté dirigé parallèlement it li surfice foliaire. Vues de face, elles sont rameuses en forme d'H.

Le mésophylle ne contient pas de cellules cristalligènes.

II. Marffe. - Lia marge est produite par la terminaison latérale du parenchyme et de l'épiderme.

III. Te'ru'r' - L'epiderme, dont les cellules sont allongées dans le sens de la nervure, est pourvu. comme celui de la lane, de poils ordinaires et glanduleur. Il enveloppe du collenchyme, du tis:u cellulaire et des faisceaux libéro-ligneux semblables ì ceux de la plupart des Borraginées.

FAMILI.E DES GENTIANEES.

Ményantle trèfle d'eau. - Menyanthes trifalinta. L.

I. Lane interneurale a. Epiderne. - L'épiderme muni de stomates et dépourvu de poils et de glandes it ses deux faces, forme une seule assise de cellules i membrane mince, irrégulière, polygonale ou légùrement ondulée. L ess stomates, gros, arrondison elliptiques, sont disposés irrégulièrement et situés entre trois on plusieurs cetlules épidermiques.

b. Vésonhllle. - Le mésophylle suporieur vert est composé de deux it tro:s conches de cellules cylindriques un peu iréculières, de deux fois à deux fois et demie plus longues que larges et allongées perpendiculairement à la surface foliaire. Le mésophylle inférieur, généralement plus développé que le précédent. est formé d'un amas de cellules rameuses laissant entre elles de petits méats. C'es cellules sont orientées de manières différentes; sur une coupe transrersale, elles sont irrégulieres.

II. Marge. - La terminaison du parenchyme vert recouvert d'épiderme constitue la marore.

III. Nervure. - Lin nervure est limitée par de l'épiderme sins sto. mates dont les cellules sont allongées dims le sens de la nervure. Fn dedans de lépiderme existe une masse de tissu dans lequel existent rénéralement trois cordons libéro-liçnenx. Les éléments qui composent cette masse (pl. YI. ligr. VI, e) se présentent en coupe transversale solls la forme de cercle ou de polygone assez irrégulier. En coupe longitudinale, ils sont rectangulaires en général, de deux ì quatre fois plus longs que laroes. Ces collules renferment dans leur intérieur quelques grains de chlorophrille et laissent entro elles de vastes lacunes (jui, on section transveruale, sont polygonales. Ces lacunes sont séparées les unes des intres par une sonde conche cellubirs.

Les cordons liberro-ligneux (d. f) sont au nombre de trois. Ils sont directement enveloppós diune assise concentrigue de cellules polyennales

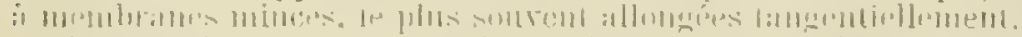

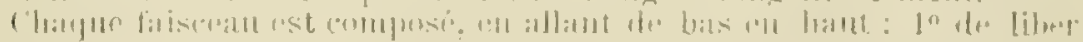

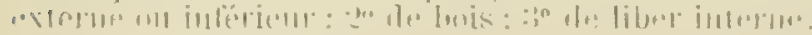

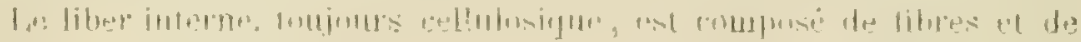


cellules disposées en un amas continu. I an section transwersaln cio cre

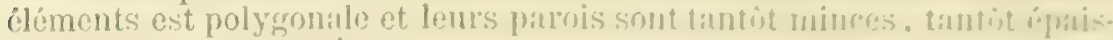
sies, sultout alux incres.

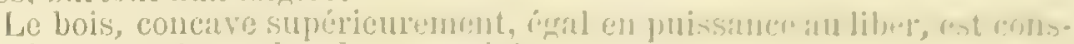

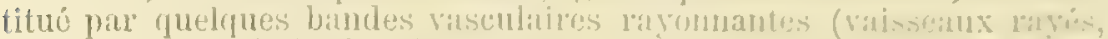

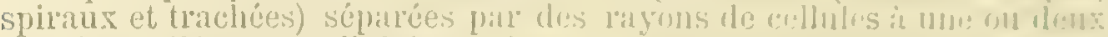

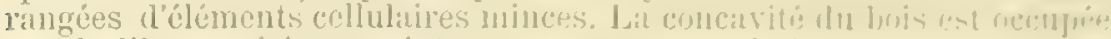

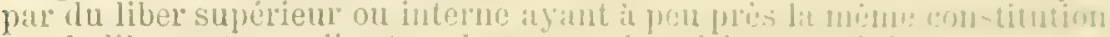
que le liber externe, il est senlement moins diche cu cellules.

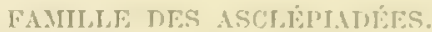

\section{Tylophora esthmatica. Wight et Arnott.}

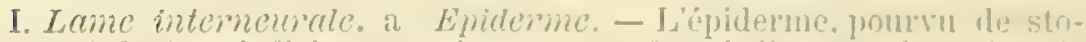
mates di la face inléricure seulennente est formé d'une senle ascise do: cellules tabulaires it membrane assc\% épaisse, it contoul polvounal. L. stomates elliptiques sont environnés de cellules épirlermìues ront deux latérales ont la forme de croissant concave il lat paltie interne.

Cet épiderme donne naissance id des poils plus ou moins lonrs. pluricellulanes, uniscóiés; leur menbrane, un peu épaissie uf ponctuée. est recouverte superficiellement d'une couche assez cinisse de cuticule.

b. Mésophylle. - A lat fixce supéricure existent deux il trois couches de grandes cellules cylindriques presseos les mos contro les antres et disposées en palissade. Elles sont en général denx à trolí finis plus longues que larges. Au-dessous d'elles vicnt une lame de cellules plus ou moins rameuses qui, en coupe tranversale, paraissent arondics ou rec-

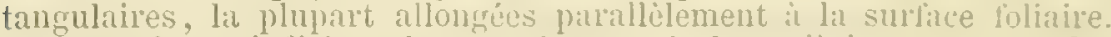
Ce parenchyme inférieur donne naissance it des cellules contenant des cristaux agoolmérés d'oxalate de chaux.

1I. Marge. - I ha marre est uniquement constituée par du parenchyme vert bordé d'épiderme.

III. Nérvurc. - En dedans d’un épiderme. dépourvu de stomaltes et composé de petites cellules ì membrane épaisse. irrérulièrentut pulygonale, un peu plus longues que latres, se troure mu masit de cellules

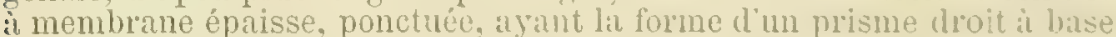
légèlement polygonale. Ces éléments, d'autant plus lizrores at moins longs qu'ils se rapprochent du centre de la nervure, laissent entre eus de petit méats. Quelques-uns renferment des cristaux arrorlomélés d'oxalate de chaux.

Un arc libélo-ligneux siège dans cet amas de parenchrme. Il est composé de liber supérieur, de bois et de liber inférieur.

Le liber externe, toujours cellulosique, est constitué par des ilots d’éléments a membrane mince, dont les uns, externes, sont fibreur: les autres, plus internes, celluleux. Les fibres sont plus ou moins rrosses et leur membrane est assez mince. Au pourtoul du liber existent de fins laticifères ramitiés.

Le bois, plus épais que le liber, est loz"mẻ de bandes lógulières ravonnantes de vaisseatux assez espacécs et séparéc entre clles ordinaire. ment par deux ou trois langées ratiales de cellules i parois minces.

Quant au liber interne, aussi développe que le liber externe: il est disposé en îlots et présente les mèmes éléments que ce dernier. 
Feuille de Solenostemme arghcl. Arghel. - Solenostemma arghel. Hayne.

I. Lame interneuralc. a. Epidemane. - L'épiderme, muni de stomates aux deux faces, est composé d'une assise de cellules tabulaires à contour polygonal; leurs parois sont recouveries superficiellement d'une couche très épaisse de cuticule, qui présente des crêtes saillantes. Les deux faces produisent des poils coniques dont la longueur dépasse plusieurs fois la largeur; ces derniers sont composés d'une série de deux à quatre cellules juxtaposées et leurs parois un peu épaissies sont garnies de tubeicules arrondis.

b. Mésophylle. - Le mésophylle constitue aux faces inférieure et supérieure plusieurs couches de petites cellules cylindriques pressées les unes contre les autres et perpendiculaires à la surface de la feuille. Entre les lames de tissu en palissade supérieure et inférieure se trouvent quelques couches de cellules allongées parallèlement à l'épiderme. Le mésophylle renferme surtout à sa partie moyenne, de gros cristaux agglomérés d'oxalate de cluaux.

II. Marge. - La terminaison latérale du mésophylle et de l'épiderme compose la marge.

III. Nervu' ${ }^{\circ}$. - L'épiderme, dépourvu de stomates et muni de poils, est composé d'une couche de cellules, qui, examinées de face, sont rectangulaires, allongées perpendiculairement à l'axe de la nervure. A l'iıtérieur de la nervure est placé un arc libéro-ligneux ayant très peu d'épaisseur. Il est composé inférieurement de liber dont les éléments cellulosiques sont disposés en îlots. Au-dessus du liber est un arc lignneux constitué par plusieurs bandes de trois à cinq vaisseaux. séparées les unes des autres par deux rangées parallèles de cellules à membiane assez mince. A la partie supérieure du bois, existe du liber disposé en îlots et ayant la même constitution que le liber inférieur.

L'arc libéro-ligneux est encastré dans une masse cellulaire, dont les éléments incolores ont la forme de prisme droit à base polygonale; ils laissent entre eux des méats el renferment la plupart des cristaux agglomérés. Ce massif, en contact avec l'épiderme inférieur, esi, au contraire, séparẻ de l'épidermesupérieur par quelques couches de cellules à chlorophylle, ovales, ou elliptiques, qui se continuent latéralement avec le parenchyme vert supérieur de la lame.

Espèce mélangée au Séné, et pouvant être substituée à ce dernier.

FAMILLE DES APOCYNEES.

Pervenche naine. Tinca minor. L.

I. Lame interneurale. a. Eniderne. - L'épiderme supérieur est composé de cellules à membranes assez épaisses. Elles sont sinueuses superficiollement. Sur une coupe transversale, elles sont rectangulaires. allongées dans le sens de la surface. Leur paroi superficielle est munie d'une cuticule assez épaisse On ne trouve point de stomates.

L'épiderme inférieur est semblable au précédent, mais il est muni de stomates elliptiques situés entre deux cellules latérales à parois un peu ondulées. 


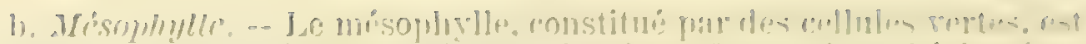

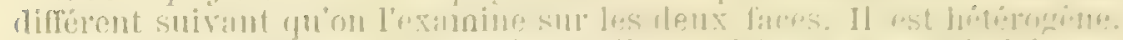

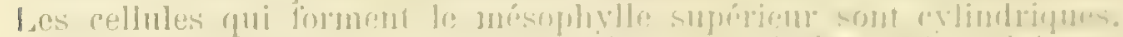

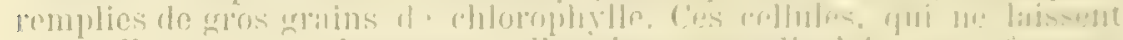

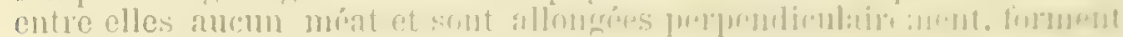

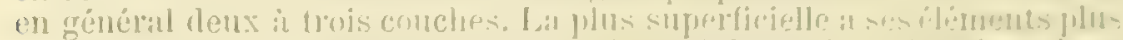

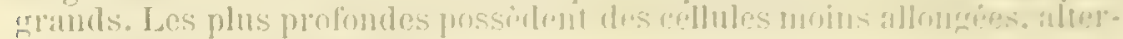
mant itrec les jomicmers.

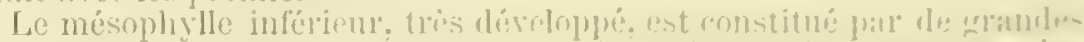

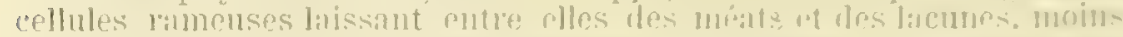

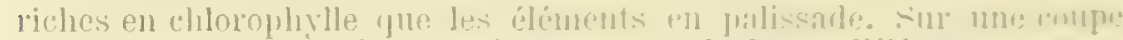

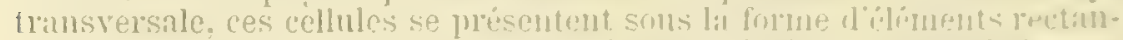

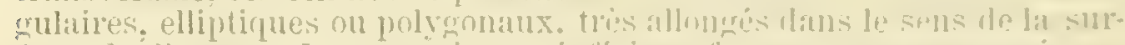

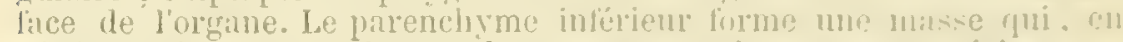
vémél:al, dépasse en puissance la masse parenchymatense supévioure.

II. Margo. - Sous l'épiderme, on remargute denx at trois innclies de cellules cylindriques it memblanes épaisce et tres refrimente. Cest contre ces éléments lrypodermiguns gue vient s'appuyer la terminaion latérale du parenchyone rert.

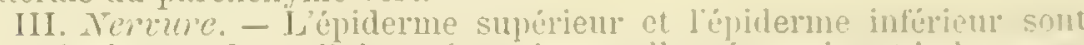
constilués par des cellules prismatiques allongées suivant la longurmo

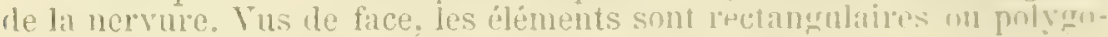

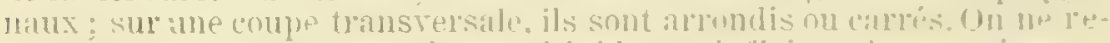

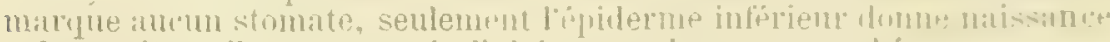

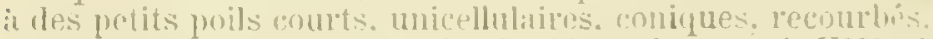

Contre l'épiderme s’appuie de l'hyporterme (pl. VIII, lïr. I. II. n) constitué par des cellules in parois trés épaisses et pourvaes le ponctuations. Les cellules hypodermigues. qui ne latissent $*$ nt $1^{\circ}$ dles aucun méat. ont la forme d'un cylindre allongé snivant lil longueur de lil nevire et remermant quelques rrius de chlorophylle. Lihyorlerme.

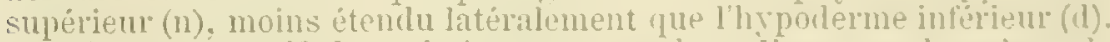
constitue un massif de trois it quatre conches. Il en est de meme de linypoderme inférienr. Entre les deux lypodernes, on trouve du paren. chyme vert dans lequel plongre le systene libéro-ligneux.

Les cellules du parenchyme touchant l'hypoderme infërieur snnt polyédriques ou arronties, transversalement. et rectangulares. allongees, sur une coupe longitmale. Lems parois presentent des ponctua. tions. Ces cellules se réunissent pour former un amas de trois it six couches dont la supérieure touche le corlon tibro-rasculaire.

Les cellules du parenchyme supérieur (m), beaucoup plıs riches en chlorophylle que les précédentes. sont au contraire, beaucoup plus petites. Sur ulie coupe longitudinale, elles sont carrées ou bien rectangulaires. allongées d'avant en arrière.

Le système libéro-ligneux qui se trouve a milieu du parenclivmc vert est un cordon de forme orale ì grand diamètre latéral. Il est constitué inféricurement par un arc de liber externe; puis d'un arc de bois ot d'un liber interne de forme ovale, placé dans un concavité qie forme l'arc supérieur ligneux.

Le liber externe cst constitue infirieurement par th faihles ilots (a) de libres cellulosiques, très grandes, macrées. i membrane tres épirse. I lans certains cas, toutes les fibres ont leur piroi mince en formant alors un ar. continu. I In limito inferirure du liber on renronts e des tules laticiferes (b), 
charriant de fines granulations. Au-dessus de ces fibres vient le puissant liber celluleux (c) elı forme d'arc. Formé d'ilots d'éléments très pelits remplis de granulations et d'albuminoildes, ces groupes séveux sont séparés radia'ement les uns des autres par des éléments ì membrane mince, mais beaucoup plus considérables.

Le bois a la forme d'un arc (b). Il a la mème épaisseur que le liber externe. Il est constitué. en allant du bas vers le haut: $1^{0}$ de fibres courtes très épaisses, carrẻes ou polygonales sur une coupe transversale. Cies fibres présentent de très pelites ponctuations et des marques spiralées ; 20 de vaisseaux cannelés, spiro annelés, spiraux et trachées. Tous ces éléments sont disposés en files rayonnantes très rapprochées.

Le liber interne $(g, h)$ a la mème constitution générale que le liber externe.

$$
\text { Grande Perrenche. - rince maim. I. }
$$

Se distingue du ronce minor par la présence de poils unicellulaires. coniques à la marge.

FAMILLe DES IOBELLACÉES.

Lobélie entléc. - Lobelia inftata. L.

1. Lame internemole. a. Éjiderme, - L'épiderme constitue unc assise de cellules tabulaires. Celles de la face inférieure ont un contour tres sinteux, tandis que celles de la face supérieure sont irrégulièrement polygogonales ou présentent de faibles ondulations.

L'épiderme inférieur est pourvu de stomates elliptiques, environnés de trois a quatre cellules épidermiques.

Des poils se rencontrent sut' les deux faces. Ils sont coniques et unicellulaires. Leur paroi, un peu épaissie, offre parfois à sa surface de petits tubercules.

b. Mésopluylle. - Iu-dessous de l'épiderme inférieur se trouve une couche de parenchyme en palissade, dont les éléments cylindriques sont deux ou trois fois plus longs que larges. Ce tissu est en contact avec une lame cellulaire. Les cellules qui la constituent forment trois à quatre couches parallèles. Vues sur une coupe transversale, elles sont allongées parallèlement à la surface de la feuille, tandis qu'elles sont irrégulières et rameuses de face.

II. Marge. - Dans certaines portions de la feuille, la terminaison du parcnchyme vert recouvert d'épiderme, constitue le bord foliaire. Mais dans d'autres parties il existe des glandes i la marge. Celles-ci forment au bord de la feuil!e des proéminences coniques incolores. Sur une coupe transversale, elles sont arrondies ou elliptiques. Elles sont composées d'un grand nombre de petites cellules polvédriques entourées de deux à trois couches concentriques de cellules aplaties radialement.

III. Nérvirc. - Lépiderme présente des poils qui ressemblent à ceux de la lame. Les cellules épidermiques sont allongées suivant l'axe de la nervure, et munies de parois un peu épaissies et ponctuées.

frans l'intéricur do lit nervure se trouve un corlon libéro-ligneux rqui a la forme d'un croissant, dont la contavité est tournée vers la face suné. rieure de la feuille.

Le liber est prosenchynateux inférieurement, celluleux supérieurement 
Les fibres libériennes ont leur membrane épaissie aux angles, nacries el cellulosiques. Les celfules dit lifer parenchrynatcux sont petites.s. allonges dans le sens de la nerviure.

Au milieu d'elles se troweni des canaux laticiferes, disposio d'ordinaire suivant une ligne courlie, imequliere. it concavite inférienre. Lies vaisseaux renferment un suc qui se coirule sous laction de la polasse, cen formant une masse qui parait grisidre atu microscope. Ils s'etendent le long de la nervure en produisant des branches laterales qui relient entre eux les canaux voisins

Le bois est constitué par des lames irféro-supérieures de vásseaux, spiraux. ponctués et de trachées. Ces lames sont séparées par des bandes de une i deux rangées cellulaires.

Le lirneux forme une concavité supérieure, occupée par un anas de cellules d'aspect collenchymatcux. Ces dernieres sont prismatiques à base polygonale, elles sont épaissies, surtout aux angles. Au-ılessus de ce jarenchyme, se trouve un massif d'éléments plus grros, laissant entre eux des méats. Un tissu semblable existe entre l'épiderme inféricur et le faisceau libéro-ligneux.

FAMILIE DES DIPSACEFS.

Scahieuse succise. - Scribiosn succisa. L.

I. Lame intemenale. a. Epiderne. - Lëpiderme, muni de sto. mates ì ses deux finces, est formé d'une senle assise de cellules tabulaires yui, examinées de face. présentent une membrane épaissie. lérérement sinueuse. Les membranes latérales sont pourrues de ponctuations allongées perpendiculairement ì la surfince foliaire. Ces deux éphilermes sont recouverts d'une conche movenne de cuticule et donnent naissance à des poils ordinaires et ì des poils glanduleux.

Les poils longs, s'amincissant de la base au sommet qui est airu. ont une membrane trés épaisse et sont supportés par une saillie cunique multiceliulaire de lépirlorme. Les poils glanduleux (pl. III. fir. IV) sont très courts, unicellulaires, it peine plus longs que larges: ils servent de support à une glande rectangulaire, it angles amondis et deux fois plus longue que large. Eille est composée de deux séries parilleles rerticales de 2,4 cellules séparées par des cloisons horizontales.

b. Mésophylle. - Le mésophylle est chlorophyllien, il ditrère sur les deux faces. I la face supérieure, ses cellules sont formées de deux couches en palissade. Elles sont à peu près cylindriques ou un peu étranglées vers le milieu de leur longueur. Les plus superdicielles sont plus longrues que les profondes, qui, en général, ont une longuem qui ne dépasse pas deux fois la largeur, tandis que les premières ont leur plus gr'and diamètre double du plus faible. Le mésophylle inférieur, en gcénéral plus développé que le supérieur", a des cellules rameuses laissant entre elles de nombreux méats et étagées en assises paralléles it l'épiderme. Vues en coupe transicrsale, elles sont arondies ou rectangrlaires, allongées dans le sens de la surface foliaire. Tues de face, elles sont rameuses, le plus souvent à quatre branches cour es disposées en $\mathbf{H}$. Dans la couche profonde du parenchrme supérieur existent des cellules arrondies contenant des cristaux agglomérés d'oxalate de chaux.

II. Marge. - La marge est formée par du parenchyme et de l'épiderme. 
III. Nervure. - La nervure est bi-convexe. Elle est recouverte d'épiderme glanduleux et relu i collules longues. ct nunies sur leur membrane ipaissic de ponctuations. Au-dessus de lépiderme inférieur siège une masse de grosses cellules à membranes épaissies, ponctuées et laissant entre elles des méats. Ces cellules, d'autant plus grindes qu'ellos sont plus voisines du centre, sont arondies ou lécèrement polygonales on coupe transtersale, rectangulaires, deux ou quatre fois plus longues que larges en coupe longitudinale. Les cellules les plus centrales touchent deux ou trois couches de cellules vertes à membranes plus minces, qui s'ippuient contre la contexité inférieure d'un cordon libéro-ligneux. Ces éléments sont rectangulaires on coupe transtersale et allongés parallèlement it l'arc libérien. Au-dessous de l'épiderme supéricur existe un massif de cellules semblables ì celles gui se trouvent ì la face inférieure. Quelques-unes, incolores, renferment des cristaux d'oxalate: d'autres, et se sont les plus courtes, renferment de la chlorophylle. Dans le tissu fondamental siège un ou souvent trois cordons libéroligneux en forme d'arce convexe inférieurement. concave supérieurement. Le liber, peu puissant. est de nature cellulosique. Il est constitué par des cellules allongeees dans le sens Iongitudinal.

Le bois, plus puissant que le liber", forme des bandes rayounantes fibro-rasculaires très serrées; la concavilé du bois est remplie inférienrement par de petites cellules à membranes un peu épaissies. ponctuées; les dernieres sont arrondies ou polygonales, en section transtersale: rectangulaires, trois ì cinq fois plus longues que larges, en section Ionritudinale.

\section{FAMILLE DES SYNANTHERREES OU COMPOSÉES.}

Espèces: Bardane commune. Lavpa communis. G. et Coss. - Chardon bénit. Cnicus lienedictus. - Séneçon commun. Senecio rulgaris. L. - Arnique des montagnes. Arnica montance. L. - Tanaisie conmune. Tanacctum rulgare. L - Armoise absinthe. Artemisia absinthium. L. - Armoise commune, Artemisia rulgaris. L Achillée milloteuille. Achillea millefolium. L. - Inule aurée. Inela helenium. L. -I. conyze. I. ronyza. D. C. - Eupatoire Aya Pana. Eupatorium dyr Panr. Vent. Tussilarre pas-d'ine. Tussilago farfara. L. - 'Tus. petasite. Tus. petasites. - Pissenlit. Taraxacum dens leonis. Desf. - Chicorée sauvage, Cichorim intyurs.

Les feuilles médicinales appartenant à cette famille, présentent une lame dont l'épiderme est variable. Les cellules de ce dernier ont leurs parois tantôt minces (Armoise, Innie), tantôt épaissies (Aya Pana, Amica); elles sont revêtues extéricuı urent d'une cuticule plus ou moins épaisse, qui offre dans certaines espèces (Epiderme supérieur de T'ussilago farfirrn. L.) (pl. I, fig. II) des crètes saillantes se présentant sous l'aspect de stries lorsqu'on examine de face un lambeau épidermique. Le conlour des cellules varie avec les espèces, et dans une même espèce, avec la tace foliaire. Dans un grand nombre de plantes, les éléments épidermiques ont un contour sinueux sur les deux faces de la feuille (Arvich. Trenacelum. Traraxncum, etc.). Les deux épidermes de l'Aya l'ana ont leurs cellules

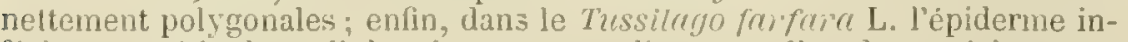
férieur possède des cellules sinueuses, tandis que celles du supérieur ont leur surface en forme de polygone.

Les stomates, jamais disposés en séries régulières, sont placẻs entre plusieurs cellules épidermiques. Toutes les feuilles, à l'exception de celles du Tussilago petasites, offrent de ces appareils sur les deux faces. 
Ia plupart des composies présentent des poils i leur surface, ceprendant dans certaines esperees ils tombent de hunne heure. Cres proils sont toujours pluricellulaires; Je plus souvent ils sont anticulés, quelpuedisis plurisériés (Cichorium. pl. II, fig. II). Leeur's formes, trits variables, sont asse\%

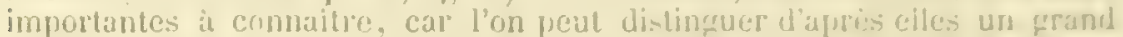
nombre de genres. Dans les dilmimisin (pl. I, fire. III, b), ils sont composis d'une portion superrjeure horizontale, unicelluláre et terminere en pointe a ses deux extrémilés. Cette partie s'insére en son unilieu sur un pédicule vertical formé d'une rangée de petites cellules crlindrifues. Darıs les lunlre (pl. I, fig. I) ils sont coniques. et lit cellule supérieure conique est rentlee it sa base. Ces formations épidermiques sont composées d'une rangée d'étéments sphériques, ovoides ou ellipsoidaux dans le I'rurre'min 'pl. III. fig. I). C'est sous la forme d'un long lil que se présente la cellule sujérieure des poils des t'ussilaryo.

Quelques espèces sont pourvues de glandes externes. Ces dernières sont sessiles ou portées par un pédicelle asse\% lon composé de plusieurs rangées cellulaires ( 1 mmict montan $)$. La base des grlandes est elliptique dans les Inulr. Lappa, delemisir. Lorsqu'on examine au microscope un fragment d'épiderme inférieur de ces especes, les glandes se présentent sous la forme d'une ellipse divisée en deux portions par une cloison perpendiculaire au grand axe. Quand on ohserve au contraire la coupe transrersale de ces feuilles, ces appareils se montrent constitués par deux rangées rerticales de deux ou plusieurs cellules (pl. I, figg. III, et pl. II, fig̣. .1).

Le mésophylle offre de nombreuses variations sur lesquelles nous ne dirons que quelques mots.

La plupart des espèces ont un parenchyme hétérogène asymétrique: d'autres, au contraire, lossèdent du tissu en palissade sur les deux faces, (Tandrétum, Achilléa). Le mésophylle est toujours dépourvu de cellules cristalligenes et de glandes internes.

Le bord foliaire est en général constitué par du tissu rert recouver d'épiderme; il a'y a d'exception que pour l'A y/r Pana qui ollre contre son épiderme marginal une conche de grandes cellules incolores et épaissies.

La nervure mérite d'occuper lattention, il catuse des particularités importantes qu'elle présente.

Dans les grosses nervures, il existe plusieurs cordons toujours séparés entre eux par de grandes cellules poiles it parois minces. Chaque cordon a le plus sourent la forme (coupe transrersale) d'une ellipse a thand diamètre inféro-supérieur (pl. VII. fig. V). Il est composé de bois at de

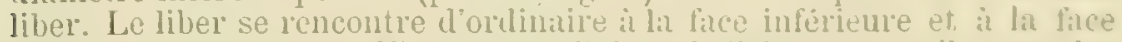
supérieme du filisceau. Fibreux et cellulenx infériemenent, il est seulement prosenchymateux supériemrement. Les fibres constitucnt un amas continu plus ou moins considérable; clles ont leur paroi cellulosique ou lignifiée. Le bois est composé de raisseatux sourent disposés irréruliérement. Il forme un massif convere à sa partie inférieure.

Le systeme fibro-rasculaire est enveloplé d un parenchyme à grandes cellules dans lequel siogent des éléments qui renferment soit du latex. soit de l'oléo-résine. Ce sont des laticifères et des canaux sécréteurs.

Les laticifères sont voisins du liber; ils samastomosent entre eux (Cichorium, Taraxucuin).

Les canaux sécréteur's sont des conduits dont la section transrersale est losangique, (Tussilago) ou polygonale (Artemisia). Ils sont bordés par une couche concentrique de quatre (Tussilago farfara) (pl. VII, tig. V. 
e, i) ou de cinq i sept cellules (Amtemisia, Aya Pana). Ces canaux sont toujours en rapport avec les faisceaux libéro-ligneux. Tintôt ils sont sitnés ì la face infërieure de chaque faisceau, tantot à la face supérieure; quelquefois ils existent sur les deux faces. Dans l'A rmica, le Tanacetum, l'Achillen millefolinm. L., les canaux sont inférienr's et au nombre de deux pour chaque coldon. Ils sont placés à l'extrémité de l'are libéricn. l'un ì droite, l'autre ì gache. Uu seul canal, situé au dos de chaque faisceau libérien, existe dans le Sénccon. Il y en a au contraire deux ou trois contre le liber de Tussilago petasiles. L. et Tussilago farfara. L. (pl. VII, fig. VI, i, e).

Les petites nervures de l'Artemisia absinthium. L. ne possèrent qu'un canal qui se trouve toujours ì la face supérieure du faisceau. Enfin le liber de l'A ya pana présente de ces réservoirs au dos el au ventre de chaque faiscenu. Il y en a un it la face rentrale; deux ou trois à la face dorsale.

Feville de Barlane commune. - Lrapua communis, Germ. et Cosson.

I. Lreme internerurele. a. Elinleme.-L'épiderme, pourvu de stomates. de poils ordinaires et de poils glanduleux sur les deux faces, est formé d'une assise de cellules tabulaires. Les cellules de l'épiderme inférieur ont un contour très sinueux, tandis que celles du supérieur présentent de faibles ondulations ou une surface polygonale.

Les poils, très nombreux à la face inférieure, rares à la supérieure, sont composẻs d'une série cellulaire.

Les cellules inférieures sont larges, et ont une longueur qui ne dépasse pas cing fois la largeur. Celles-ci supportent une cellule filiforme très allongée.

Les glandes sont constituées par deux rangées verticales, composées chacune de trois à six cellules séparées par des cloisons horizontales. Ces glandes paraissent elliptiques lorsqu'on les examine par leur sommet; elles semblent ovales à grosse extrémité supérieure, lor:qu’on les observe de profil. Flles sont soutenues par un pédicelle unicellulaire très court (pl. II, fig. VI).

b. Irésophylle. - Tous les éléments situés entre les deux membranes épidermiques, renferment de la chlorophylle; aucun ne présente de cristaux dans leur cavité. Une couche de cellulos cylindriques, perpendiculaires à la surface foliaire, $1^{2}$ drux ou trois fois plus longues que larges, est voisine de l'épiderme supérieur. Au-dessus de ce parenchyme en palissade sont de deux à trois assises parallèles de cellules qui, sur une ioupe transversale, paraissent allongées parallèlement à l'épiderme.

II. Nervuri? - La nervure est bi-convexe. Son épiderme, poilu et glanduleux, constitue sur les deux faces une couche de cellules tabulaires allongées suivant l'axe neural. Contre lui s'appuient deux à quatre couches de cellules collenchymateuses.

Entre le collenchyme supérieur et le collenchyme inférieur, est placée une masse parenchymateuse qui enveloppe quelques cordons libéro-ligneux. Les cellules du parenchyme sont assez volumineuses. Leurs parois sont minces. Elles s'oftrent sous l'aspect de polygones assez réguliers lorsqu'on examine une coupe perpendiculaire à l'axe de la nervure. Ces cellules pâles, laissent entre elles de petits mẻats.

Quelques faisceaux libéro-ligneux sont situés dans ce tissu. Ils forment 
enscmble un are convexe inféricurement, concave supéricurement. Charque cordon présente une section transversile à peu pries colliptique. it frand diamètre infero-supérieur. On trouve, en allaut du bas vers le ladut, Jes tissus suivants :

$1^{\circ}$ Du liber fibreux dont les éléments ont une membrane nacréce ípais-ie el cellulosirque;

: isu jiber celluleux;

:3" Du bois convexe a sa partic supérieure, et composé de gros vaisseaux disposés irrégulierement;

10 Un massif de prosenchyme, semblable à celui du liber inféricur.

Charlon hénit. - Cnicus hruchliotus. L.

I. Lame inlemenorale. a. Fipiderme. - J'épiderme est composé d'une conche de cellules tahulaires it contour sinueux: il est dípourru de stomates aux deux faces et parzemé de Inngs poils composés d'une série verticale de cellules dont la longueur va d'ordinaire en augmentant de la base all sommet. Il donne aussi naissance it de courts poils bicellulaires dont les cellules sont séparées par une cloison transversale et supportent des glandes composées de deux séries verticales de cellules plus larges que hautes et limitées par des cloisons horizontales.

b. Mésojhylle. - Le mésophylle constitue à la face inférieure et it la face supérieure deux couches de cellules allongées perpendiculairement it l'épiderme, et, en général, trois ì quatre fois plus longues que larges. Entre ces couches existe souvent une assise de cellules plus petites ordinairement, allongées parallèlement in l'épiderme.

II. Marge. - La marge est formée de cellules vertes recourertes d'épiderme.

III. Tervure. - La nervure a un épiderme is cellules dont les mem. branes sont un peu épaissies. Vues de face, elles ont la forme de polygone allongé parallèlement à la nervure. L'épiderme, dépourvu de stomates, est muni de poils semblables i ceux qui existent sul la lame. Au-lessus du parenchyme infërieur existent quelques couches de cel!ules collenchỵmateuses. Entre ce collenchyme et l'épiderme se troure un amas de grandes cellules qui laissent entre elles des méats; leurs membranes. assez. minces, sont munies de ponctuations, elles ont la forme de prisme droit à base polygonale. Ce tissu renferme des faisceaux libéro-ligneux, généralement au nombre de trois ct formant ensemble une espèce d'arc; chaque cordon se trouve composé à sa partie inférieure d'un amas de fibres à contour polygonal et à membrane mince ou épaissie. Au-dessus du prosenchỵme se troure le liber celluleux. Le bois, dont la section transiersale nst con. vexe supérieurement, est composé it sa partie inférieure d'un amas de fibres it contour polygonal et à membrane mince ou épaissie. Au-dessus du prosenchyme se trouve le liber celluleux. Le bois, dont la section transversale est conrexe supérieurement, est composé de quelques lames vasculaires. I la partie supérieure du bois siège un amas de cellules qui ne laissent entre elles aucun méat; leur membrane épaissie et quelquefois lignifiée prisente de nombreuses ponctuations arrondies. Ces cellules ont la forme de longs prismes droits it lase polygonale. 
Sẻneçon commun. - Senecio rulgaris. L.

I. Lame interneurale. a. Epiderme. - L'épiderme est composé d'une couche de cellules tabulaires à contour sinueux. Il est glabre et pourvu sur les deux faces de stomates elliptiques.

b. Mésophylle. - Le mésophylle forme à la face supérieure deux ou trois couches de grandes cellules cylindriques allongées perpendiculairement à l'épiderme et en général deux fois plus longues que larges; à la face inférieure se trouvent quelques couches de cellules, qui, en coupe transversale, sont dirigées en tous sens et sinueuses; de face, elles sont rameuses à trois ou quatre branches très courtes.

II. Marge. - La marge est composée de parenchyme vert revêtu d'épiderme.

III. Nervure. - L'épiderme, formé d'une couche de cellules allongées parallèlement à la nervure, est muni de stomates. Les feuilles jeunes possèdent des poils composés d'une série verticale de cellules dont la plupart sont cylindriques ou en forme de tonneau plus longues que larges; la cellule terminale allongée est conique, leur membrane est généralement peu épaisse.

En dedans de l'épiderme se trouve une masse assez considérable de grandes cellules pâles à membrane mince. Ces cellules, qui laissent entre clles de faibles méats. ont une section transverse polygonale et sont rectangulaires, allongées dans le sens longitudinal. Ce parenchyme contient généralement trois cordons dont l'ensemble forme un arc à concavité supérieure. Contre chaque faisceau, à leur face inférieure et vers la région médiane, se trouve un canal sécréteur entouré d'une couche de quatre cellules allongées tangentiellement; chaque faisceau est arrondi, ovale, elliptique à grand dıamètre le plus souvent inféro-supérieur.

A la partie inférieure se trouve le liber qui est parenchymateux à sa périphérie; ces fibres ont un contour polygonal; leur membrane cellulosiqque est mince ou quelquefois épaissie. Le liber celluleux est généralement aussi développé que le prẻcédent.

Le bois est formé de quelques bandes rayonnantes de vaisseaux dont le diamètre est égal à celui des fibres libériennes. Des rayons médullaires à une seule rangée de cellules séparent les lames vasculaires. Au-dessus du bois, se trouve un massif de cellules d'aspect collenchymateux à membrane épaissic et nacrée.

\section{Feuille d'Arnique des montagnes. - Arnica montana. L.}

1. Lame internenrale. a. Expiderme. - L'épiderme est formé d'une couche de cellules tabulaires à contour sinueux; il est pourvu sur les deux faces de stomates, de poils et de glandes. Les poils sont d'ordinaire composés d'une série de cellules plus longues que larges, et à membrane faiblement épaissie. Les glandes sont sessiles ou portées par un pédicelle assez long; les glandes sessiles sont le plus souvent enfoncées dans une dépression de l'épiderme, elles sont unicellulaires ou pluricellulaires, et, dans ce cas, elles sont composées de deux couches superposées de deux ou pl ısieurs cellules. Les glandes pédicellées sont arrondies ou se présentent de côté comme ayant la forme d'un tranèze à grand côté supérieur; un plus ou inoins grand nombre de cellules, séparés par des cloisons ver- 
ticales, horizontales el oblirques, entre dans leur constitution. Io poil conique qui soutient charque glande. est forme de plusieurs serries cellulaires.

b. Mésophogllé. - Le inésophylle forme à la face supérieure une ru deux couches de cellules cylindrigues, allongées perpendiculairement is l'épiderme: les plus grandes sont trois fois plus longrnes que larges. it la face inférieure se trouve une masse cellulaire plus dévelopjuée que le paren. chyme en palissade. Les cellules grandes, raneuses et dirigées en trous sens, laissent entre elles de grands méats.

II. Monge - La marge est uniruement constiluéc par la terninaison latérale du mésophylle et de l'épiderne.

III. Nerume. - La nervure est convexe inférieurement, excavéc supérieurement. Elle possède un épiderme muni de stomates, de glandes et de poils et est composé de cellules qui, vues de face, ont la forme d'un polygone allongées parallelement it l'axe de la nervure. Intre Jes deux epdermes se trouve une masse de grandes cellules pâles qui laissent entre elles des méats; leur membrane est un peu épaissié, elles sont arrondies ou polygonales sur une coupe transversale, et poiggonales ou rectangulaires, allongées sur une coupe longitudinale. Dans ce tissu sièggent généralement trois cordons libéro-ligneux contre lesquels se trouvent placés des canaux sécréteurs. Ces derniers, au nombre de deux pour charjue faisceau. se trouvent placé, l'un à droite, l'autre à gauche de l'extrémité de l'are formé par le liber. La section transverse de chaque cordon est ovale ou elliptique is grand diamètre quelquefois latéral, souvent inféro-supérieur ; ì sa face inférieure existe un massif de fibres dont les membranes sont épaissies, ponctuées et d'ordinaire lignifiées: leur contour est polygonal et arrondi, leur cavitẻ est assez grande. Quant au liber celluleux, il est composé de petites cellules. Le bois, concave en haut, est forme de plusieurs landes vasculaires irrégulières de vaisseaux. Au-dessus du hois se trouve un amas de cellules dont les membranes sont épaissies, ponctuées et lignifiées, elles ne laissent entre elles aucun méat et ont la forme de longs prismes droits it base polysonale. Ce petit amas celluleux touche supérieurement quelques fibres semblables à celles du liber inférieur.

Tanaisic commune. - Tenacetum vulgare. L.

1. Lame internowrale. a. Epiderince. - L'épiderme est formé d'une seule couche de cellules tabulaires à contour sinueux. Il est pourru de stomates elliptiques aux deux faces et donne naissance a des poils et il cles glandes. Les poils pluricellulaires (pl. III, fig. I) sont composés d'une file de cellules elliptiques it peine deux fois plus longues que larges, disposées sur un seul rang. Les glandes enfoncées dans les dépressions de l'épiderme sont composées de deux rangées verticales chacune de deux ou trois cellules séparées par des cloisons horizontales; rues de face, les glandes sont elliptiques.

b. Mésophylle. - Le mésophylle forme à la face inférieure et à la face supéricure une ou deux couches de cellules allongées perpendiculairement it la surface; les plus grandes sont de cinc à six fois plus longues que larges; les supérieures sont nettement cylindriques, les inférieures ont un contour sinueux; entre ces deux couches se trouvent trois assises de ceilules àe forme rectangulaire allongées parallèlement ả l'épiderme. 
II. Marge. - La marge est uniquement constituée par la terminaison latérale du mésophylle et de l'épiderme.

III. Yervure. - L'épiderme neural, pourvu de stomates, de glandes et de poils, est composé d'une couche de cellules allongées parallèlement à l'axe de la nervure; vues do face, elles sont polygonales, rectangulaires et leur membrane épaissie présente des stries dues ì de petites crêtes. En dedans de l'épiderme se trouve un massif plus puissant de collenchyme en dedans duquel siège une masse de grandes cellules à parois minces et à contour polygonal. Cet amas, qui contient un cordon libéro-ligneux, produit des canaux sécréteurs situés au voisinage du faisceau. Ils sont placés au nombre de deux, l'un à l'extrémité droite, l'autre ì l'extrémité gauche de l'arc libérien. Le faisceau est ovale sur une coupe trásversale et son grand diamètre est perpendiculaire ì la surface épidermique. Son liber est formé à sa partie inférieure d'un massif assez puissant de fibres à. contour polygonal et à membrane le plus souvent épaissie, cellulosique et lignifiée. Au-dessus du parenchyme vient le liber celluleux moins développé que le précédent.

Le bois, concave supérieurement, est formé de quelques bandes rayonnantes de plusieurs vaisseaux, séparées par une ou deux rangées de cel. lules; à la partie supérieure du bois se trouve un petit massif de fibres qui présentent la même constitution que les fibres du liber inférieur.

Feuille d'Armoise absinthe. - Artemisia absinthium. L.

I. Lame interneurale. a. Epiderme. - L'épiderme est formé d'une couche de cellules à membrane mince. de forme tabulaire ì contour sinueux. Cet épiderme, muni de stomates sur les deux faces, présente ¿i la face supérieure et a l'inférieure de nombreux poils et des glandes. Ces poils (pl. I. fig. III, b) sont constitués par une longue cellule supérieure, horizontale, terminée en pointe à ses deux extrémités. Au milieu de sa longneur s'insère un pédicello cylindrique, composé d'une rangée de petites cellules superposées. Les glandes (a, i) sessiles ou portées par un pédicelle extrêmement court, se trouvent logées dans une excavation de l'épiderme. De face, elles sont elliptiques; de profil, elles ont la forme d'un dôme arrondi ì son extrémité. Chaque glande est généralement composée de deux séries verticales, de deux ì trois cellules séparées entre elles par des cloisons horizontales. Elles contiennent une huile essentielle jaunitre.

b. Mésophylle. - Le mésophylle constitue à la face supérieure une ou deux couches de cellules cylindriques de deux it quatre fois plus longues que larges; ces cellules, assez serrécs les unes contre les autres, sont dirigées perpendiculairement ì la surtace foliaire. A la face inférieure, il existe un parenchyme ì méat, généralement plus puissant que le parenchyme supérieur; ces cellules sont rameuses, celles qui touchent le tissu en palissade paraissent, sur une coupe transversale. allongces parallèlement ì l'épiderme; celles qui sont voisines de l'épiderme inférieur, sont la plupart perpendiculaires à la surface.

II. Marge. - La marge est uniquement composée de parenchyme vert reconvert d'épiderme.

III. Nervune. - La nelvule, bi-convexe, est limitée en haut et en bas par. un épiderme dépourvu de stomates, et muni de poils et de glandes; ces éléments sont allongés parallèlement ì l'axe de la nervure. Au-des. 
sus de l'épiderme supérieur se trouvent ruelques couches do crllules

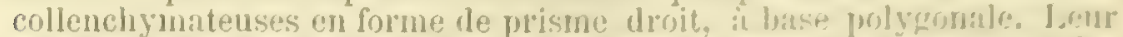
membrane est mince; si ce nost anx andrles, ril elles jurescutent dos épaississement qui font le plus sonvent saillie dans la cavito cedlulairo.

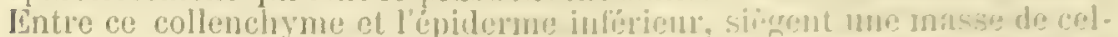

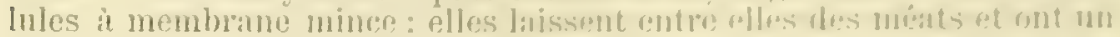

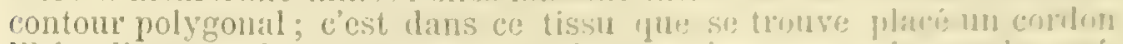
libéro-ligneux. On rencontre atussi daus ce tissu un petit canal sucréteur, existant le plus souvent at la face supérienro, et tont contre le sy.steme fibro-rasculaire. Ce canal, bien visible surtout dans les petiles nervures, a une section transversalo qualdanchlates at il est entoure d'une rangée de quatre, le plus sonvent de cind it sis cellules disposées concentriquement antour de lui. Le liber est tris développe; il a ses éléments petits, minces et cellulosirnes.

Le bois, beaucoup moins dévoloppé que le liber, présente une con. vexité tournée vers la face supérieure de la feuille: il est formé de plusieurs bandes rayonnantes de vaisseaux. dont lo diannetre ne dépasse guère deux fois le diametre des grosses cellules libériennes. Les lames vasculaires sont séparées les unes des autres jak des layons d'une rangée de collules. I la partie supérieure du bois on lencontre un amas de cellules dont la forme est celle d'un prisme droit à base polygonale: ces cellules ne laissent entre elles aucun méat et possielent des parois assez minces.

Feuille d'Armoise commune. - Artemisia rulgaris. L.

LiArtemisia rulgaris se distingue du précédent par l'absence de poils sur la face supérieure.

Achillée millefeuille. - Achillea Mrillefolium. L.

I. Lame internourale. a. Epiderme. - L'épiderme est composé d'une assise de cellules tabulaires it contour sinueux. Il est recourert d'une couche de cuticule crétée et pourvu aux deur finces de stomates, de poils et de glandes. Les poils couchés sur l'épiderme sont multicelluInires et les cellules qui les composent sont superposées en une série verticale. La base du poil, formée d'une gl’ande cellule en forme de cône tronqué, soutient une ranrée de quelques petites cellules cylindriques dont la largeur est généralement plus grande que la hauteur. La cellule supérieure supporte une très longue cellule conique àt membrane tris épaisse. Les glaudes sessiles ou presquue sessiles. sont enfoncées dans une dépression de l'épiderme. Elles sont bicellulaires et leur's éléments sont séparés par une cloison verticale. Vues de côté, elles ont la forme d'un trapèze ì grand côté superficicl; de face, elles sont elliptiques.

b. Mésophylle. - Le mésophylle oflie ì la fince supérieure et à la face inférieure une assise de cellules cylindriques allongées perpendicularirement à l'épiderme: entre les deux couches en palissade supérieure et inférieure il en existe une ou deux dont les éléments sont le plus souvent allongés parallèlement à la surface.

II. Marge. - La marge est uniquement constituce par la terminaison latérale de l'épiderme et du mésophỹlle. 
III. Nervurc- La nervure, bi-convexe, présente un épiderme pourvu de stomates, de poils et de glandes semblables id ceux qui existent sur la lame; les ce!lules qui le composent sont allongées parallèlement à l'axe de la nervure. En dedans de l'épiderme inférieur siègent deux ì trois couches de cellules collenchymateuses ayant la forme d'un long prisme droit à base polygonale. Les épaississements que présentent ces cellules à leur's angles ne sont pas considérables; au-dessous de l'épiderme supérieur se trouvent deux à trois couches de cellules munies de chlorophylle, se continuant arec les cellules supérieures du mésophylle.

Entre ce parenchyme vort et le collenchyme inférieur existent un amas de grandes cellules ì contour polygonal, à membrane mince, elles laissent entre elles des méats, et sur une coupe longitudinale, elles ont la forme d'un rectangle allongé parallélement à l'axe de la nervure. Ce tissu renferme un coldon libéro-ligneur et produit au voisinage de ce cordon de petits canaux sécréteurs semblables à ceux qui existent dans les Composées. Ces canaux, au nombre de deux, sont situés l'un à droite, l'autre à gauche du cordon aux extrémités de l'arc libérien. Ils sont polygonaux et entourés de quatre à cinq cellules sécrétrices. Le faisceau, dont la section transverse a la forme d'un ovale ou d'une ellipse ¿t grand diamètre d'ordinaire inféro-supérieur. se compose, en allant du bas vers le haut: $\mathbf{1}^{0}$ de liber fibreux. inférienr ou externe, dont les éléments il contour polygonal ont leur membrane épaissie, le plus souvent cellulosique, quelquefois lignifiée; $2^{\circ}$ de liber celluleux moins développé que le précédent; $3^{0}$ de bois moins puissant que le liber et formé de quelques bandes vasculaires de trois à cinq vaisseaux chacune, séparées entre elles par des rayons médullaires à une seule rangée de cellules; $4^{\circ}$ d'un massif de fibres qui présentent la même constitution que celles du liber inférieur.

Inule conyze. - Inula conyza. D. C.

I. Lame interneurale. a. Epiderme. - Son épiderme (pl. I, fig. I) est composé d'une conche de cellules tabulaires ¿̀ parois présentant un contour sinueux. Il est pourvu aux deux faces de stomates, de poils et de glandes. Les poils droits sont composés d'une série verticale de cellules d'autant plus longues qu'elles approchent du sommet. La base de chaque cellule, élargie, s'amincit à quelque distance pour devenir plus large an sommet; leur paroi est un peu épaissie. Les glandes sessiles ont la forme de ceintre et sont composées de deux séries verticales de plusieur's cellules séparées entre elles par des cloisons horizontales et qui sont plus larges que hautes,

b. Mésopllylle. - Le mésophylle constitue à la face supérieure une couche de cellules cylindriques allongées perpendiculairement à la surface et deux fois plus longues que larges; ¿̀ la face inférieure se trouvent trois in cinq couches de cellules assez serrées, qui, sur une coupe transversale, semblent arrondies, carrées ou rectangulaires; tanulis que de face, elles sont rameuses.

II. Marge. - La marge est constituée uniquement par la terminaison latérale du mésophylle et de l'épiderme.

III. Ne'rure. - L'épiderme de la nervure poilu, glanduleux, mais dépourvu de stomates est composé de cellules, qui, vues de face, ont la 


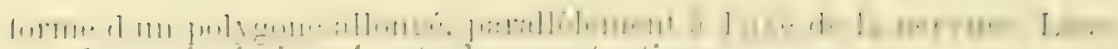
menblane épilissie présente des ponctuations.

Au-dessus de l'épiderme inférieur se tronvent qualdues conches de longues cellules collenchymateuses, prismatinues. it base polyomale:

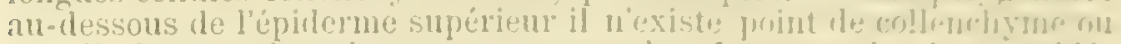
bien il nest représenté que par une couche. Lil natse la julus consulérable de la nervure est forméc d'un parenchyno lont les cellules priles et it membrane asse\% inince sont polygonales tant smo une conje trablsversale que sur une coupe longitudinale. Dans re tis-n sierrent diondinaire plusieurs cordons libéro-lignenx disposés en arc: chargne corrlon est composé de liber infériem, de hois et de liber sujéripon. Le liber inférieur est fibrenx it sa périphérie; ses ćléments. qui forment un massif assez considérable, ont un contour polyonal. Len membane est épaissie, cellulosique et lenr cavité ent assez grande. Aurlessus est la masse de cellules libériennes moins développée que le prosenchyme.

Le bois, en général plus puissant que le liber, est composé dle vais. seaux disposés en lame rayonnantes, séprées par des cellules it parois épaissies, nacrées.

Quant au liber supérieur, il n'est représenté que par des libres qui présentent la mème constitution que celles du liber inférieur.

Inule aunée. - Inula helenium. I.

L'Inule aunée se distincrue du précédent par es lcellules supérieures du mésophylle qui sont sphériques ou elliptiques, fiblenent allonées. et par la membrane des cellules de l'épiderme inférieur qui n'est pas ponctuée.

Feuille d'Eupatoire Aya Pana. - Eupatorizin Aya Pana. Vent.

I. Lame intemenrale. a. Elviderme. - L'épiderme est composé d une seule couche de cellules tabulaires i contour fablement sinueux ou polyronal. Les deux épidermes sont pourvus de stomates: on remarque it l'inférieur des poils composés d'une série de cellules sipperposées presque aussi larges que longues. La cellule supérieure est arrondie $\mathfrak{h}$ son sommet.

b. Mésophylle. - Le mésophylle forme à la face supérieure deux ou trois couches de cellules eylindriques: les unes sont aussi larres que longues, les autres ont une longueur qui ne dépasse jamais deur fois la largeur. A la face inférieure, il constitue deux ì quatre conches paral. lèles de cellules allongées qui, en conpe transtersale, sont rectangulaires, allongées parallẻlement it la surface de la feuille; de face, elles sont polygonales, sinueuses ou faiblement lameuses.

II. Marge. Contre l'épiderme marginal s'appuie une couche de cellules assez grandes et épaissies.

III. Nervure. - L'épiderme neural. dépour'vu de stomates et garni de petits poils analogues a ceux que l'on remarque sur la lame, est formé d'une conche de cellules ayant, de face, la forme de ploygone nu le rectangle allongé parallèlement í l'axe de la nervure. En dedans de l'épiderme se trouvent deux ì quatre couches de cellules collenchymateuses ayant la forme de prisme droit it base polygonale. Ces cellules sont épaissies aux angles. En dedans du collenchỹme sière une masse de 
tissu cellulaire dans laquelle est placé un ou trois cordons disposés en un arc concave inférieurement, convexe supérieurement. Les élẻments qui constituent ce parenchyme ont une membrane mince, un contour polygonal, sont disposés en file longitudinale, et sont séparés par des cloisons horizontales. On remarque dans ce tissu des canaux sécréteur:s situés tant ì la face supérieure qu'à la face inférieure. Ces canaux quadrangulaires, en coupe transversale, sont entourés chacun d'une couche de quatre i six cellules disposées concentriquement. Ils sont voisins des cordons libéro-ligneux. Lor'squ'il existe plusieurs faisceaux, le médian présente inférieurement trois canaux : l'un médian, les deux autres latéraux; supérieurement il n'en offre qu'un seul. Chaque cordon est composé à sa partie inférieure de liber fibreux. Les fibres sont le plus souvent minces, quelquefois cependant leur membrane est trẻs épaissie et lignifiée. Le liber celluleux. placé à la face supérieure du fibreux, a ses éléments très petits.

Le bois, en général égal au liber, se compose de quelques lames rayomnantes de trois i six vaisseaux assez gros ; ces lames vasculaires sont séparées par des rayons inédullaires composés d'une ou deux rangées de cellules deux à trois fois moins longues que les vaisseaux; audessus du bois siège un amas de cellules à membrane mince qui ne laissent entre elles aucun méat et ont la forme de prisme allongé ì base polygonale.

Feuille de Tussilage pas-d'àne. - Tissilago farfara. L.

I. Lame interneurale. a. Epideme. - L'épiderme est formé d'une couche de cellules tabulaires. Les cellules de l'épiderme inférieur ont un contour sinueux, tandis que les cellules supérieures (pl. I, fig. II) l'ont nettement polygonal. Ces dernières sont recouvertes, ì leur suftuce. d'une cuticule qui présente des crètes assez saillantes. Des stomates se rencontrent sur les deux faces seulement, ils sont assez rares i la face supérieure. L'épiderme inférieur donne naissance à de longs pouls, composés chacun d'une série verticale de cellules, dont les inférieures sont lectangulaires ou en forme de tomneau, tandis que la supérieure est très longue et filiforme. Le mésophylle forme, à la face supérieure, deux a trois couches de grandes cellules cylindriques, dont les profondes ont une longueur qui ne dépasse pas deux fois la largeur; la couche supérieure a ses cellules beancoup plus allongées. A la face inférieure, se trouvent des cellules disposées en couches parallèles, superposées très courtes; vues sur une coupe transversale, elles paraissent carrées ou rectangulaires, allongées parallèlement à la surface.

II. Marge. - La marge est composée de mésophylle vert revêtu d'épiderme.

III. Nervure. - La nervure est bi-convexe. Son épiderme est composé d'une couche de cellules ì membrane faiblement épaissie, présentant superficiellement quelques stries; vues de face, elles sont rectangulaires ou polygonales et sont allongées parallèlement ì l'axe de la nervure. L'épiderine est dépour'vu de stomates, mais garni de poils semblables it ceux qui existent sur la lame. En dedans de l'épiderme, siègent des cellules collenchymateuses, ayant la forme de long prisme à base polygonale. Ces éléments présentent aux angles de forts épaississements, qui, la plupart, s'avancent daus la cavité cellulaire. Entre le 


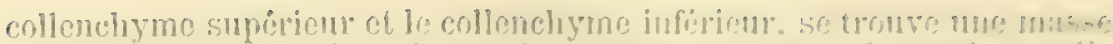

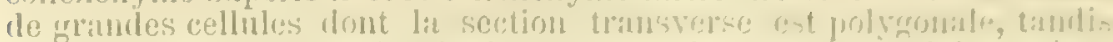

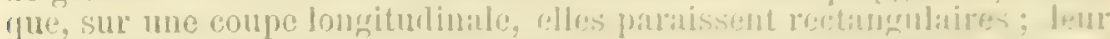

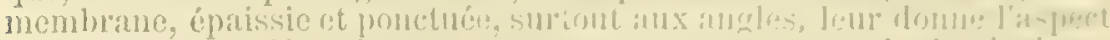

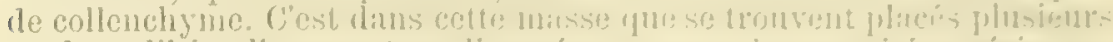

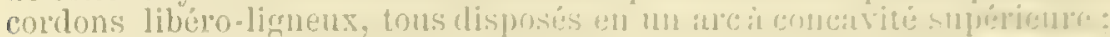

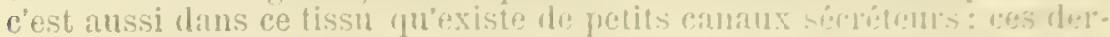

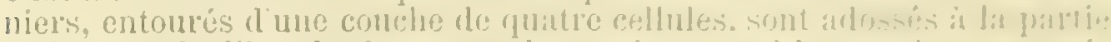

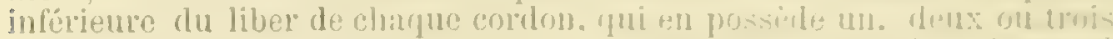

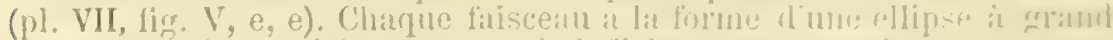

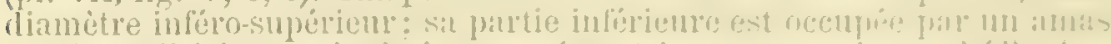

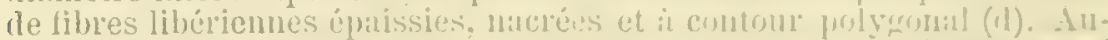
dessus du paronchyme, vient le liber celluleux (c), en crobial ansi puissant que le précédent. Le bois (b), dont lid section transirerse at la forme d'un croissant convexe supérienrerement. est compróé lu bandes rayomantes irréguliùres de vaisseanx issez gros; ces laimes sont séprarées par des cellules it contomr polyonal et un peu ipaissi anx anyles. Lu-dessus du bois siège un massif de libres semblables a celles du liber infërieur (b).

Tussilage pétasite. - Tussilago petasites. L.

Le Tussilago officinalis se distingue du Tussilago forfirie: $1^{\circ}$ par les cellules de son épiderme supérieur dépourvu de stomatos ct composé de cellules dont le contonr est lérèrement sinueus: 20 par lit disposition de ses faisceaux. Ces dernier's sont disposís en plusieun's ares. les uns supérieurs, les autres infërieurs.

Chicorée commune. - Cichorium intybus. L.

I. Lame intomeurale. a. Elpillerme. - L'épiderme est composé d'une couche de cellules tabulaires it contour sinuenx, il est pourvu de stomates et de poils aux deux faces; les poils, longs et coniques, sont formés de plusieurs cellules dispostés en plusieurs rangées rerticules (1). II, Hi: II .

b. Mésomhylle. - Le mésophylle vert a ses éléments d'ordinaire tous allongés perpendiculairement à la surface de la fenille, seulement î la face supérieure ils sont plus considérables, nettement cylindriques et forment deux couches: les inférieur's sont cylindriques ou thliptiques: les autres sont le plus souvent sinueux.

II. Marge. - L'extrémité du parenchyme et de l'épiderme constitue lat marge.

III. Nenvure. - L'épiderme de la nervure forme une couche de collules dont les membranes, épaissies, présentent de lécgeres ponctuations. Leur surface extérienre est munie de stries dues it de légéres crêtes; vues de face, elles ont lia forme de polygones irriguliers. allongés parallèlement á l'axe de la nerrure. En dedans de lépiderme se trouve une couche de cellules collenchymateuses. La masse principale est formée d'un parenchyme it grandes cellules piles il contour polygonal ou arrondi et allongé suivant la nervure: elles laissent entre elles de nombreux méats. Cet amas renferme plusieurs cordons libéroligneux disposés en un arc convexe infẻrieurement, concare supérieu- 
rement. Contre les corlons et $a$ lenr face inferrieure se voient des tubes remplis d'un latex blanchittie. Chaque fuisceau est ovale à grand diamètre d'ordinaire inféro-supérieur. Sa partie inférieure libérienne est composée à sa périphérie d'un amas continu de fibres à membrane épaissie, nacrée et cellulosique à cavité grande et à contour polygonal. Au-dessus du prosenchyme sièrrent des cellules libériennes.

Le bois, égal en puissance an liber, office une section transversale, biconvexe ou plan-convere. Il est formé de quelques bandes rayonnantes de deux ì huit gros vaisseaux dont le diamètre est plus grand que celui des fibres libériennes. Les lames vasculaires sont séparées les unes des autres par une ou deux rangées de cellules à membrane mince. Audessus du bois se trouvent quelques cellules dont le contour est polygonal et qui sont allongées parallèlement à l'are de la nervure. Ces cellules touchent supérieurement un amas de fibres analogues à celles du liber inférieur.

Pissenlit dent de lion. - Taraxacum dens leonis. Desf.

I. Lame interneurale. a. Epiderme. - L'épiderme est composé d'une assise de cellules tabulaires à contour sinueux, il est glabre et pourvu de stomates sur les deux faces.

b. Mésophylle. - Le mésophylle est formé à la face supérieure de cellules ovales on elliptiques, ou sphériques, dont la longueur' n'atteint pas deux fois la largeur. A la face inférieure existent plusieurs couches dont l'ensemble est en général plus développé que le par'enchyme en palissade. Les cellules qui constituent ces couches sont rameuses superficiellement et leur's branches sont très courtes.

II. Marge. - La marge est uniquement constituée par le parenchyme vert recouvert d'épiderme.

III. Nervure. - La ne. Nura est bi-convexe. Son épiderme, pourvu de stomates, présente parfois quelques poils coniques, unicellulaires. Les cellules de l'épiderme ont un conton polygonal, elles sont disposées en séries verticales et sont séparées les unes des autres par des cloisons horizontales et obliques. En dedans de l'épiderme existe quelquefois une couche de cellules collenchymateuses.

La masse principale de la nervure est formée d'un parenchyme méatique n'existant pas au centre de la nervure où se trouve une grande lacune en forme de canal. Les cellules qui composent ce parenchyme sont grandes, polyédriques et ont leurs inembranes minces; elles sont pâles. Ce tissu renferme plusieur's cordons libéro-ligneux dont les uns, p'us gros sont disposés en are ì la face inférieure, tandis que les autres, plus petits, forment un arc supéricur. Chaque faisceau est ovale ou elliptique à grand diamètre, tantôt latéral, tantôt inféro-supérieur. Sa partie inférieure est constituée par du liber fibreux ì sa piriphérie; les fibres forment un massif continu disposé en arc, elles ont un contour polygonal et leur membrane, épaissie, nacrée et cellulosique entoure une cavité centrale assez grande. Quant au liber celluleux, il est moins développé que le précédent. Contre la partie inférieure du liber se remarquent des vaisseaux laticiferes scmblables à ceux que l'on tiouve dans la nervure de Cichorium.

Le bois, convexe à sa partie supérieure, est formé de plusieurs bandes convergentes et régulières de quelques vaisseaux; au-dessus du bois siège un petit amas d'éléments semblables aux fibres du liber inférieur. 


\section{C:IINITITLE IV}

\section{CLEIF DICHOTOMLUE.}

Pour rendre rapide la détermination histologique dı limbe des feuilles utiles ì la médecine et pouvant être substituées à celles-ci, nous avons eu recours à une méthode qu'un grand nombre de bota-

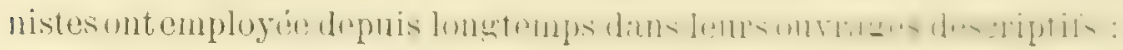
nous voulons parler de la clef dichotominue. Nous nous sommes attaché à établir ce système d'après des caractères microscopiques dont on peut sans trop de peine constater la présence, et nous arons tåché de grouper ensemble les espèces appartenant à la même famille ré-

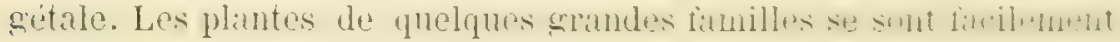
prêtées à un tel arrangement. Dans ce cas, sont les direrses feuilles des Labiées, des Synanthérées, des Borraginées, des Rutacées, ctc. Une semblable disposition montre qu'il y a une grande ressemblance entre les espèces d'un mème groupe, et que l'on peut arriver à la connaissance de la famille à l'aide d'un ensemble de caractères histologiques.

Stomates à la face supérieure seulement. Fibres à ponctuations

1 aréolées dans le bois. ............ 己

Stomates aux deux faces ou à la face inférieure. Point de fibres à ponctuations aréolées . . . . . . 3 3

Paroi des cellules situées entre le faiscean et l'ipiderune supérieur, minces . . . Juniperus sabina. L.

Paroi des cellules situées entre le faisceau de l'épiderme supérieur, épaisses . . . Junipenus virginiana. L.

3 Des cystolithes sphériques dans les cellules épidermiques ......... Parietaria officinalis. L. Point de cystolithes. . . . . . . . . . 
( Vaisseaux laticifères dans leliber mou. Lobclia m flata. L.

1

Point de vaisseaux laticifères dans le liber

Des glandes pluricellulaires dans le parenchyme vert de

5 Des glandes unicellulaires dans le parenchyme vert de I la lawe. . . . . . . . . . . . . 14

Point de glandes . . . . . . . . . . . . 18

6 Une couche de parenchyme vert en palissade . . . . 7

( Deux ou plusieurs conches de parenchyme en palissade. 11

Point de fibres libériennes ou fibres cellulosiques.

7 Plelea trifoliata. L.

bes fibres lignifies . . . . . . . . . . . 8

Un arc libéro-ligneux inférieur. Face profonde des cellules épidermiques supérieures se gonflant sous l'influence de l'enu . . . . . . . . . . . .

Deux ares vasculaires l'un inférieur, l'autre supérieur. Face profonde de cellules épidermique non mucilagineuse. . Pilocarpus pennatifolius. Lem. Jaborandi

Des poils unicellnlaires à l'épiderme superieur de la nervure. De nombreuses cellules cristalligènes dans le mésophylle . . . . . . . . . . . . .

Point de poils, point de cellules cristalligènes.

Empleurum sermlatum. Ait.

Cellules épidermiques recouvrant les dents de la marge très épaissies et très ponctuées.

Cellules épidermiques peu épaissies, peu ponctuées.

Barosma serratifolia. Wild.

Cellules en palissade sur les deux faces. Cellules épider1:1 miques non sinueuses.... Exucalyptus globulus. Lab. I Des cellules en palissade à une face. Cellules épidermiques sinueuses. . . . Myrlus communis. L. 
Un arc vasculaire inféricur, flores libériennes collulo1.; 1 siques. . . . . . . . . . . . . 1:3 bis

f Denx ou plusieurs aies vasculaires, l'un inférienr, les illtres supérieurs, fibres lignifiées. Citrus vulgaris. Iit.

1:3h. Cellules épidermiques sinueuses. Ruer grarealérs. L.

Point d'assises de cellules incolores an-dessous de l'épi14 , derme ........... Laumen nobilis. L. Assise de cellules incolores au-dessous de l'épiderme. .

Poils en rosette à l'épiderme. l’oint de glandes dans le 15 liber ......... Pneumus Loldus. Mol. Point de poils en rosette. Glandes dans le liber...

Collenchyme disposé en faisceau dans la nervure. Poils 1 articulés. . . . . Artanthe clongala. Miq. 16 Point de collenchyme, ou collenchyme continu. Point de poils articulés. . . . . . . . . . . . . . .

Fibres lignifiées au-dessus du collenchyme inférieur. Cellules du tissu en palissade à peine deux fois plus Iongues que larges . . . Serronia Jaborandi. Point de fibres. Cellules du parenchyme en palissade au moins deux fois aussi longues que larges.

Piper reticulatum. L.

18 Des glandes ou canaux sécréteurs dans le liber ... . 19

Point de glandes . . . . . . . . . 20

Faisceaux libéro-ligneux inférieurs très espacés.

19 Faisceaux libéro-ligneux très rapprochés de manière à I à former un arc à concavité supérieure.

Rhus coriainia. L.

$20 \int_{1}^{\text {Mésophylle vert moins épais que les deux épidermes }}$ Mésophylle toujours plus developpé que l'épiderme. . 
Du liber supérieur celluleux ou cellulo-fibreux à élé1 ments cellulosiques, disposé en îlots au moins supérieurs à trois.

I Point de liber supérieur, on liber supérieur non disposé en îlots ou formant seulement un ou deux îlots . . . :33

(Des stomates à une seule face . . . . . . . . 23

$22\{$ Stomates aux derx faces . . . . . . . . . . . 25

$23\left\{\begin{array}{r}\text { Cellules épidermiques à contour non sinueux. } \\ \text { Tylophora asthmatica. Wight et Arnot. }\end{array}\right.$

Cellules épidermiques à contour sinueux . . . . . . 21

24 Poils à la marge........ Tinca major. L.

Point de poils à la marge . . . . Vinca minor. L.

Epiderme à cellules non sinueuses.

25 Solenostemma Arghel. Hayne. Epiderme à cellules sinueuses. . . . . . . . 26

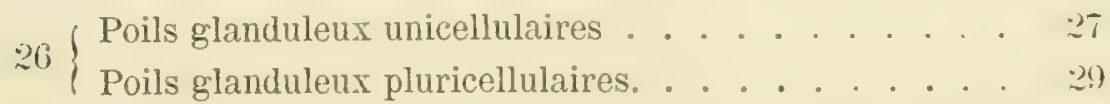

$27 \begin{aligned} & \text { Point de cellules à cristaux dans le mésophylle. } \\ & \text { Solanum nigmom. L. }\end{aligned}$

( Des cellules à cristaux dans le mésophylle. . . . . . . 28

Glandes ovoïdes. Des cristaux pulvérulents dans le mésophylle......... Atropa belladona. L.

28 Glandes ayant la forme de cine trunqué à grande lase

I supérieure. Des cristaux agglomérés.

Datura stramonium. L.

Cellules inférieures du parenchyme inférieur perpendi$29\left\{\begin{array}{l}\text { culaires à la surface épidermique . . . . . . . . . } \\ \text { Cellules inféricures du parenchyme parallèles à la sur- }\end{array}\right.$ face épidermique . . . . . . . . . . 31

Cellules cristalligènes du mésophylle renfermant la pluisolés. . . . . . . Hyoseygmes nigor. L. 


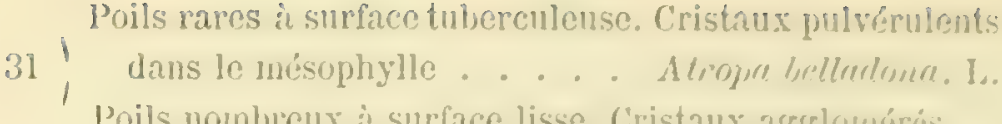

poils mombreux ì surface lisse. Cristaux agglomérés.

Une seule conche de collenchyne à la face infórienre de

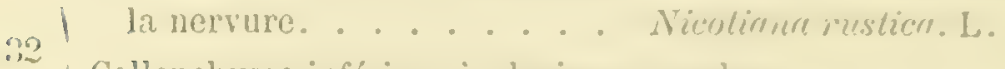

f Collenchyme inférieur à plusieurs couches.

Nicolireur laluncirme. L.

Des cellules épaissies, rameuses dans le parenchyrne de lia liılı!.

$7 \%, \ldots, \ldots, \ldots, \ldots$

33 Des cellules épaissies en forme de fibres dans le parenchyme de la lame. Point de cellules épaissies.

Angirecum fragians. D. Th.

Des cellules cristalligènes dans le parenchyme ncural ou

34 dans le mésophylle.

Point de cellules critalligènes . . . . . . . . . . 60

j Parenchyme en palissade aux deux faces .......

Deux à trois couches de parenchyme en palissade. Amas de cellules incolores entre l'épiderme supérieur et le

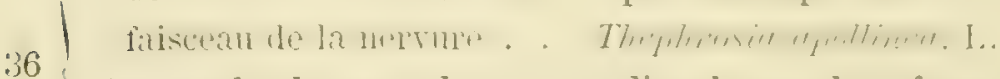

Une couche de parenchyme en palissade aux deux faces. Cellules vertes semblables à cellules du tissu muriforme au-dessus du cordon libéro-ligneux. . . . . .

| Point de cellules cristalligènes dans le mésophylle. Cel-

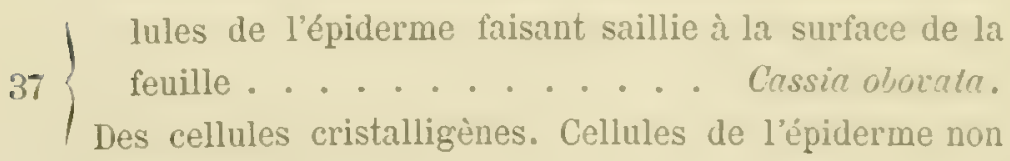
saillantes. . . . . . . . . . . . .

Poils de l'épiderme supérieur ou traces des poils séparés

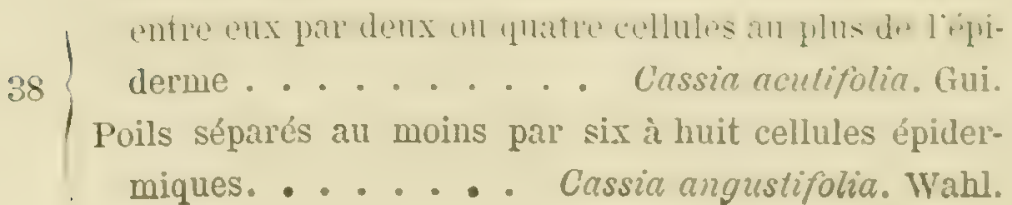


Système fondamental supérieur de la nervure homogène | à cellules semblables à celles du parenchyme supé39 rieur de la lame. . . . Buxus sempervinens. L. Système fondamental supérieur de la nervure hétérogène ou homogène à cellules dissemblables. . . . . . 4 40

40 Des canaux sécréteurs dans le parenchyme neural . . 41

Point de canaux sécréteurs . . . . . . . . 42

Des cellules pãles rectangulaires un peu épaissies à la 41 marge. Fibres libériennes souvent lignifiées.

I Point de cellules pâles à la marge. Fibres toujours cellulosiques . . . . . . Suncerlu curumun. L.

42 Collenchyme convexe dans la nervure . . . . . . . . . . Li:

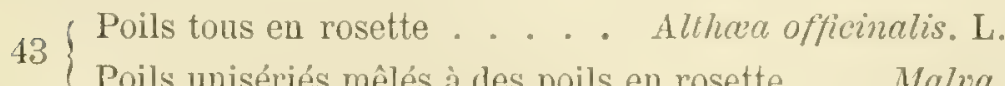

44 Stomates aux deux faces . . . . . . . . 45

Stomates à la face inférieure seulement. . . . . . . 47

$45\left\{\begin{array}{l}\text { Glandes pédicellées sur l'épiderme inférieur, dont les } \\ \text { cellules sunt punctuées . . . Mórliusr suc'cisł. L. } \\ \text { Point de glandes. Cellules épidermiques non poncutées. }\end{array}\right.$

Epiderme neural recouvert de poils unicellulaires. Faisceau libéro-ligneux entouré d'une couche de cellules

46 protectrices, et ayant la forme d'un arce à extrémités

46 supérieures très r’nprochées. Alchemilla vulgaris. L.

Epiderme glabre. Faisceau libéro-ligneux, non entouré de conche protectrice. Les deux extrémités sont très icartées........ Sonmurnir culyumis. L.

47 Une couche de cellules en palissade . . . . . . 48

\{ Deux ou plusieurs couches de tissu en palissade . . . . 49

1 Cristaux isolés en parenchyme neural. Cellules épider$45 \mid$ miques, non sinueuses . . Erythraxylon coca. L. f Cristanx agglomérés. Cellules épidermiques sinmeuses. Ribes nigrum. L. 
Un ou plusieurs ares libero-ligneux, tourmant lemr bois

49 supérieurement .............

I)eux ares libéro-ligneux, l'un supérieur, l'antre inférienr

( Des glandes externes. Cellules inferienres du mésurbylle rameuses sur une coupe transtersile de la fouille.

Point de glandes externes. Cellules inférienres du inésu-

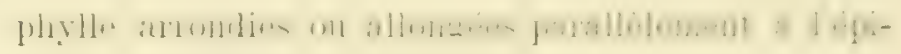
derme, sur une coupe transiersale.

' Fibres lignifiées du liber, isolées. Cellules du parenchyme

| neural, voisines du faisceau libéro-lignenx, renfermant des cristaux isolés d'oxalate de chaux.

Fibres libérienues en couches centinues. Cellules contenant des cristaux agglomérés. Ilex paraguryensis L.

Une assise de cellules incolores au-dessus de l'épiderme

52 supérieure de la lame ..... Ilex aquifolium. L.

Point de cellules incolores

Glandes externes dont les unes sont supportées par une ․) I pédicelle composé d'une ou denx rangées de trois à f sir cellules. . . . . . Ledum palustice. L. Point de glandes externes . . . . . . . . .

Système fondamental inférieur homogène ou hétérogẻne

j1 mêlé, à cellules petites et épaissies mélangées à des 1 cellules grandes et minces. . . . . . . . . . is

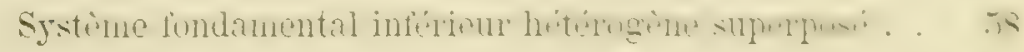

_ i Fibres libériennes lignifiées. . . . . . . . . . . . . if

Fibres libériennes non lignifiées. . . . . . . . . . 5

Point d'éléments lignifiés a la marge. 56 Andromeda polizfolia. L.

| Des éléments lignifiés à la marge.

Gaultheria pocumbens. L. 
$57 \begin{aligned} & \text { Cellules épidermiques à contour sinueux. } \\ & \text { Chimaphila mmellata. Nutt. }\end{aligned}$

581 Cellules épidermiques sinueuses. Des poils étoilés sur la 1 Cellules non sinueuses

Cellules épaissies incolores à la marge.

59 Cellules vertes non épaissies à la marge.

Perisica vulgaris. Mill.

60) Massif périphérique neural lacuneux à lacunes séparées

I Massif périphérique non lacuneux .........

Faisceaux libéro-ligneux plongés dans un amas d'élé61 ments lignifiés . . . . . Berbers vulgaris. L. | Faisceaux situés dans une masse cellulaire à parois cellulusiques. . . . . . . . . . . . . 62

$62\left\{\begin{array}{l}\text { Section transversale du liber mou biconvexe. . . . . . } \\ \text { Section transversale du liber en forme de croissant à con- }\end{array}\right.$

63 Epiderme glabre...... Aconilum napellus. L.

! Epiderme poilu . . . . . . . . . . 64

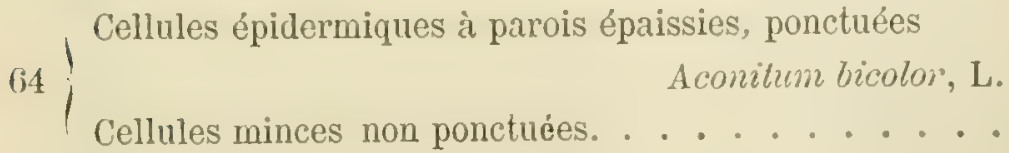

Surface extérieure des poils lisses.

65

Aconitum lycoclonum. L. | Surface extérieure des poils hérissés de tubercules.

Aconitum anthora. L.

, Poils unicellulaires à base rétrécie.

Colutea arborescens. L.

! Point de poils ou poils à base large. . . . . . . . 
Epiderme pourvu de glandes externes coniques it base inférieure, composíes de cellules séparíes par des cloisons dirigées en tous sens, et supportées par un pédicelle unicellulaire, cylindrique dont la hanteur

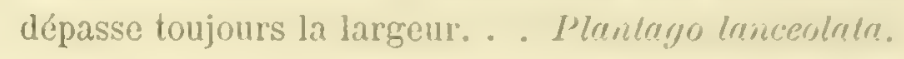

Point de glandes externes on glandes ne présentant pas ce caractère. . . . . . . . . . .

Glandes externes unicellulaires on pluricellulaires à éléments formant tous une senle couche et it pédicelle toujours unisérié. Ces glandes ont un contour arrondi.

Point de glandes of glandes dont les cellules sont disposées sur plusieurs rangs horizontanx; elles ont un contour elliptique . . . . . . . . . . In!

Poils tous unicellulaires . . . . . . . . .

69 Point de poils ou poils tous pluricellulaires ou en partie unicellulaires............... in

Epiderme pourvu de grosses glandes presque sessiles - Composées de quatre à huit cellules. Ces glandes sont 70 senles ou mélangées à des glandes plus petites unicellulaires on bicellulaires. . . . . . . . . iti

Point de glandes quadri ou octocellulaires .... . . il

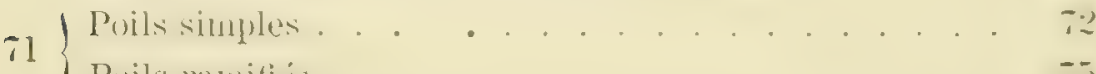

Poils lanific. . . . . . . . . . . . . .

Système fondamental neural supérieur homogène à éléments contenant de la chlorophylle. Pédicelle glandu-

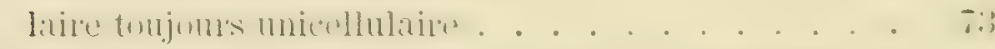

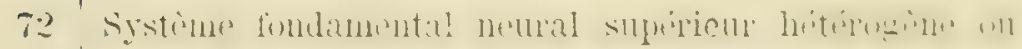
homogène à cellules incolores. Pédicelle glandulaire presque toujour's composée d'une série de deux à quatre cellules. . . . . . . . . . . 74

$73\left\{\begin{array}{c}\text { Epiderme supérieur à cellules à peine sinueuses. } \\ \text { Plunturyu muju, L. L. } \\ \text { Epiderme supérieur à cellules très sinuenses. } \\ \text { Plantago media. L. }\end{array}\right.$ 
$74\left\{\begin{array}{c}\text { Stomates sur les deux faces . Digitalis grandiflora. L. } \\ \text { Stomates à la face supérieure seulement. } \\ \text { Digitalis purpurea. L. }\end{array}\right.$

Poils présentant chacun deux ou plusieurs verticilles

75 Poils dont la plupart n'offre qu'un seul verticille, géne.

¿2. Verbascum nigrum. L.

76 Poils ramifiés. . . . . . . . . . . 77

76 Poils simples. . . . . . . . . . . . 78

Poils très longs. Point de couches de cellules incolores au-dessus de l'épiderme supérieur. Une assise de tissu 77 en palissade....... Origanum dictamnus. L. Poils assez courts. Une couche de cellules incolores. Deux ou trois assises de tissu en palissade.

Rosmarinus officinalis. L.

Fibres libériennes lignifiées. Glandes externes toutes volumineuses. presque sessiles et composées de plus 78 de denx cellules .... Fraxims excelsior. L. Fibres cellulosiques. Epiderme produisant plusieurs espèces de glandes. . . . . . . . . . . . 79

Poils longs dont quelques-uns s'insèrent ensemble au 79 nombre de deux, trois ou quatre, sur une éminence épidermique....... Marmbium vulgare. L. Poils jamais agglomérés . . . . . . . . . . 80

so Une couche de parenchyme en palissade. . . . . . 81

Deux ou plusieurs couches de parenchyme en palissade. $\quad 85$

Epiderme pourvu de poils, dont un grand nombre ont leur cellule supérieure formant un coude avec l'inférieur. La plupart des stomates sont situés entre trois ou quatre cellules épidermiques.

Tencrium chamadrys. L.
Epiderme pourvu de poils droits. Les nombreux stomates sont placés entre deux cellules épidermiques. . 
Poils de l'épiderme inférieur", la plupart longs et compro82 sés d'une rangée d'an ruoins quatre collules.

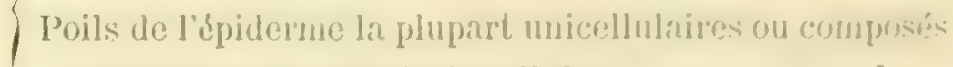
d'une file de derix it trois cellules. .

Petites glandes ovoldes ì grosses cextromitus inférienres. Epiderme supéricur muni de poils comrts micellulatres môlés à des poils plus longs mniscriés. presque tous composés de deux ì quatre cellules.

Nejela calaivir. L.

Parenchyme en palissade, égal en puissance au parenchyme inférieur. Poils bi-cellulaires courbés.

Poils tous droits. . . . . . Melissa officinalis. L.

(Cellules du mésophylle dirigées toutes perpendiculaire-

s.) ment à la surface foliaire............

fellules du parenchyme inférieur, rameuses ou allongées parallèlement à l'épiderme. . . . . . . . . .

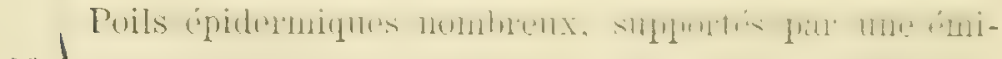

86 nence conique pluricellulaire. Glechoma hederacer. L.

Poils ne présentant pas ce caractère . . . . . . .

Base des poils très épaissie et renflée, située entre des 87 cellules épidermiques... Belonica officinalis. L.

Poil à base peu épaissie, et insérée au-dessus de l'épiderme . . . . . . . . . . . ss

Poils composés d'un rang de quatre cellules à parois lisses. Cellules supéricures du parenchyme en palissalir, à longuenr atteignant an plus denx fois la lar-

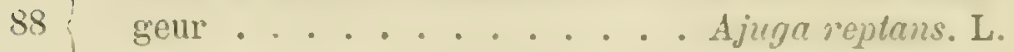
Poils courts à une ou trois cellules. Cellules du parenchyme en palissade, au moins deux fois et demi plus longues tgue larges . . . . . . . . . . 
Cellules de l'épiderme inférieur non ponctuées. Des sto-

89 cellules épidermiques. Teucrim chanaclrys. I.

fellules de l'épiderme ponctuées. Stomates sur les deux faces, presques tous placés sur des cloisons limitant

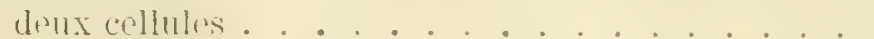

Poils de la base longs et composés de quatre à six cel-

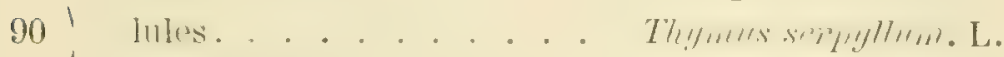

! Poils de la base courts . . . . Thymuzs vulgaris. L.

Poils courts unis ou bicellulaires non filiformes.

91 Hyssopus officinalis. L.

Poils longs au moins composés de quatre cellules ou poils filliforwes. . . . . . . . . . . . . 92

De nombreuses glandes unicellulaires supportées par un

$92\{$ pédicelle long constitué par quatre et six articles.

Giandes semblables mulles ou raves. "

Cellules épidermiques à contour non sinueux. Poils fili-

93 formes. . . . . . . . . Sulvir officinntis.

Cellules épidermiques à contour sinueux . . . . . . .

Cellules de l'épiderme inférieur de la nervure ponctuées

01 et munies de crêtes superficielles. Salvia pratensis. L.

94 / Cellules non ponctuées dépourvues de crêtes.

Salvia sclarea. L.

Glandes externes sessiles ou supportées par un pédicelle 95 unicellulaire plus large que haut .........

| Glandes externes supportées par un pédicelle pluricellulaires ou unicellulaire plus haut que large .....

Épiderme supérieur à contour non sinueux. Cellules vertes toutes perpendiculaires à la surface foliairc.

96

Lippia cilriadora. Kunth.

Epiderme supérieur ì contour sinueux. Cellules vertes; les supérieures seulement perpendiculaires à la surface foliaire ...... Verbena officinalis. L. 
97 ) Poils en hamecon. .... Symyhylum officinale. L.

Point de poils en hamecon.............

Des stomates à la face supérieure seulement. Glandes

98 supportées par un pédicelle constitúe par une file de plusieurs cellules. . . . . . . . . . . .

Stomates sur les deux faces. Glandes à pédicolle unicel. Iulaire....... Pulmonario officinalis. L.

Epiderme supérieur ì cellules peu sinueuses.

Epiderme supérieur à cellules très sinueuses.

Anchusa officinalis. L.

Poils unicellulaires sur la nervure, point de canaux $100\left\{\begin{array}{l}\text { secréteurs dans le parenchyme neural. . . . . . } \\ \text { Point de poils ou poils phurcellulaires ou }\end{array}\right.$ avec des canaux secréteurs . . . . . . . .

Fibres libériennes lignifiées. Un faisceau d'éléments épaissis et lignifiés à la marge. Parenchyme neural Fibres cellulosiques, point de faisceau à la marge. Parenchyme neural hétérogène.

Cynoglossum otficinale. L.

Faisceau libéro-ligneux entouré d'une couche continue 102 de cellules pàles, allongées tangentiellement.

Coriaria myrtifolia. L.

Faisceau libéro-ligneux non enreloppé d'uue assise protectrice continue. . . . . . . . . 103

103 Cellules, contenant de l'oléo-résine, situées cntre plu-

Point de glandes dans l'épiderme. . . . . . . .

Système fondamental supérieur de la nervure homogène, formé d'au moins trois couches de grandes cellules renfermant de la chlorophylle.

Système fondamental supérieur soit hétérogène, soit homogène à cellules incolores ou à cellules vertes disposées seulement en une ou deux couches . . . . 106 
/ Cellules de l'épiderme inférieur à membrane peu sinueuse, à sinus peu profonds. Cellules du parenchyme 105 infúrieur de la nervure, rameuses sur une coupe Cellules de l'épiderme inférieur, très sinueuses. Cellules du parenchyme vert inférieur de la lame non rameuses.

Nasturtium officinale. R. Br.

Parenchyme supérieur homogène formé d'un amas quadrangulaire, constitué par deux couches de cellules

I incolore épaissies. Parenchyme inférieur composé de

106 cellules épaissies à section transversale arrondies.

Glandes multi-cellulaire en massue, surmontant un pédicelle pluri-sérié..... Vaccinium vitis idlaa. L.

Parenchyme neural ne présentant pas ces caractères. 107 $107\left\{\begin{array}{c}\text { Poils en navette ou poils à cellule terminale longue et } \\ \text { filiforme ........................... } 108\end{array}\right.$

Point de poils laineux, point de poils en navette. . . 112

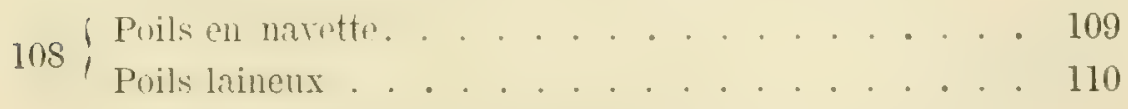

Des poils nombreux sur l'épiderme supérieur.

109 Artemisia absinthium. L.

Artemisia vulgaris. L.

$110\left\{\begin{array}{l}\text { Irenx un plusients couches de cellules mo palissade. } \\ \text { Une conche de cellules en palissade. } \\ \text { Lappa commonis. Ger. et Coss. }\end{array}\right.$

Cellules de l'épiderme supérieur à contour sinueux dé-

111 pourvues de stomates. . . Cellules de l'épiderme supsilago petasiles. L. pourvues de stomates... Tussilago farfara. L.

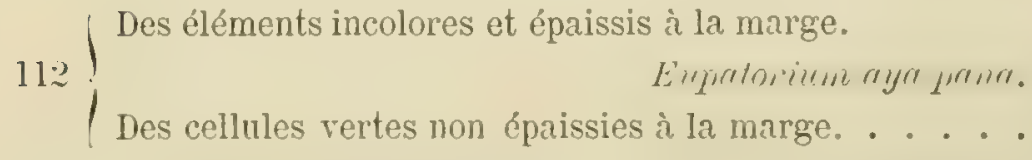

113 Poils ordinaires plurisériés. . Cichorium intybus. L.

( Pas de poils plurisériés. . . . . . . . . . 114 


\section{$-168-$}

Cellules voisines de l'épiderme superieur ot de l'epiderme inférieur perpendiculaires í la surface. Filles forment sur les deux fuces, deux larnes entre lesryuelles siègent des cellules arrondies on allongées parallielement il la surface . . . . . . . . . . .

Mésophylle ne présentant pas ces caractires. . . . .

Poils de l'épiderme inférieur de la lane et de la nervire composés d'une série de quatre ou six collules arron-

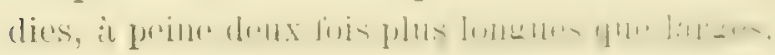

Pas de poils ou des poils ì cellule supérienre conique non arrondie. . . . . . . . . .

Poils à base conique unicellulaire, supportant un cylindre composé de un rang de trois à six cellules plus larges que longues, cylindre composé par une longue

116 pointe épaisse. Canaux secréteurs en parenchyıne

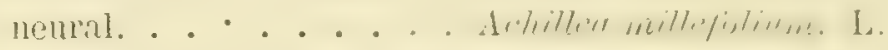

Point de poils, ou poils ne présentant pas ce caractère. Point de canaux sécréteurs . C Cnicus benedichus. L.

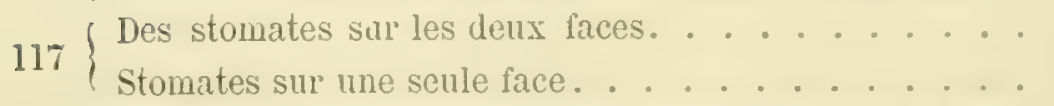

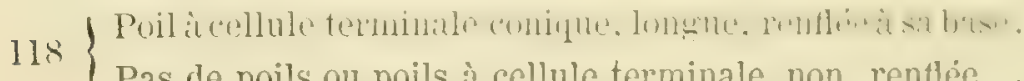

Cellules vertes voisines de l'épiderme supérieur sphéri$119\left\{\begin{array}{c}\text { que, ou faiblement allongées . . Inula helenium. 1. } \\ \text { Cellules en palissade allongées . . Inula conyzu. D. C. }\end{array}\right.$ Cellules voisines de l'épiderme supérieur, arrondies dont $120\left\{\begin{array}{r}\text { la longueur ne dépasse pas deux fois la largeur. } \\ \text { Taraxacum dens leonis I)erf. } \\ \text { Cellules voisines de l'épiderme supérieur, au moins deux }\end{array}\right.$ fois aussi longues que larges. . . . . . . 1?

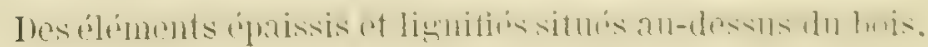
Glandes externes composées de deux rangs horizon-

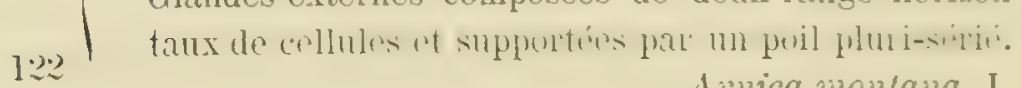

Point d'éléments épaissis au-dessus du bois ou éléments cellulosiques. Point de glandes ........ 
$123\left\{\begin{array}{l}\text { Canaux secréteurs adjacents au liber, entourés de quatre } \\ \text { cellules secrétrices.... Senecio vulgaris. L. } \\ \text { Canaux secréteurs non adjacents au liber et entourés de }\end{array}\right.$ plus de quatre cellulos . . . . . . . . . 1:24

Du collenchyme sur une face de la nervure.

Conium maculatum.

J)u collendhyme sur las lonx finces. . . . . . . 125

Deux canaux sécrétenrs pour chaque cordon libéro-

$\int$ lignenx. I, inu dursal, liantre rentral.

Apium graveolens. L.

Un canal placé au-dessous du liber.

Enanthe apiifolia. L.

Cellules en palissade en général trois fois plus longues

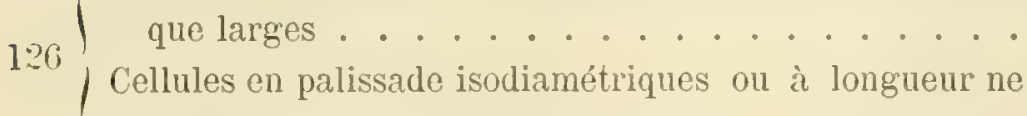
dépassant pas deux fois la largeur. . . . . . 129

De l'hyponderme à la face supérieme . . . . . . . 1:28

127 Point d'hypoderme à la face supérieure.

Conium maculatum. L.

/ Cellules de l'épiderme neural supérieur formant des saillies coniques très saillantes à membrane crêtée. Cellules épidermiques supérieures à membrane faible-

128 ment sinueuses. ....... Ciurta virosa. L.

Cellules de l'épiderme neural ne formant pas de saillies coniques; l'épiderme supérieur souvent poilu et composé de collules sinueuses . Ethusa cynapium. L.

IIypoderme sur la face supérieure de la nervure. Epiderme glabre, crêté à surface.

Point d'hypoderme sur la face supérieure. Epiderme neural souvent muni de poils nnicellulaires. Epiderme non crêté. . . . . Anthriscus cercfolium. Hoff. 



\section{CONGLUSTONS GENERALES.}

Les recherches que nous avons entreprises sur l'histologie des feuilles médicinales et de leurs falsifications se sont étendues au limbe de plus de 125 plantes. Les connaissances acquises sur ce sujet étaient très incomplètes. Les études n'avaient en effet jusqu'ici porté que sur quelques feuilles employées en thérapeutique, et les pharmacologistes et naturalistes qui se sont occupés de la question n'avaient tenu aucun compte de la constitution de la nervure. Celle-ci en raison même de sa très grande variété de structure fournit de précieux caractères pour la détermination.

Nous croyons pouvoir résumer notre travail dans les conclusions générales suivantes:

I. Il est possible de reconnaître entre elles les fenilles médicinales, et de distinguer celles-ci de leurs falsifications, au moyen des données que fournit l'examen microscopique.

II. Il est inutile d'aroir ì sa disposition des feuilles entières pour en faire une analyse histologique: quelques fragments suffisent. Nous avons toujour's eu recours à des fragments appartenant à la base du limbe et comprenant la nervure médiane, ainsi qu'à des morceaux dans lesquels sont contenus le bord foliaire.

III. La forme des cellules épidermiques et la structure de la masse générale du mésophylle sont sujettes ì de si grandes variations diuns les diverses espèces qui constituent par leur réunion une famille végétale, qu'elles ne peuvent servir à caractériser les familles: mais elles fournissent d'excellents caractères génériques et spécifiques.

VI. Les productions épidermiques (poils ordinaires, poils glanduleux), les organites du mésophylle et de la nervure (cellules cristalligènes, glandes internes, vaisseaux laticifères, canaux sécréteurs) et certains tissus neuraux (liber interne), ont une haute importance. Leur présence est constante, leur constitution générale offre souvent une remarquable fixité daus les régétaux de quelques familles. Jussi 
méritent-ils d'être appliqués a la détermination des familles on de groupements de famille.

V. Certaines fanilles viggétales, parmi lesquelles on doit ranger les Labicées, les Solanées, les Borraginées, les Synanthérées, peuvent se distinguer par leur structure micruscopique. Ces faruilles se ditrérencient en effet entre elles par un ensemble très tranché de caractires.

L'observation à l'aide du microscope a, dans ces cas une supériorité incontestable sur l'examen de l'aspect extérieur. La forme, la division et la nervation du limbe foliaire, varient tellement d'une

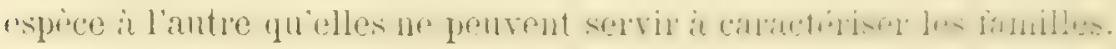




\section{TABLE DES CHAPITRES}

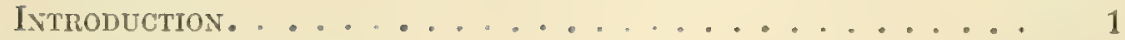

Histologie générale. . . . . . . . . . . . . 5

Technique. . . . . . . . . . . . . . . . . . . . 48

Description histologique des feutlles médcinales . . . . . 47

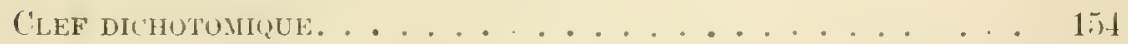

Conclustons générales . . . . . . . . . . . . . 171 



\section{INDEX ALPHABÉTIQUE}

DES

FAMILLES, GENRES ET ESPĖES

Ache orlorante....... 91

Achillea millefolium. L. . . 148

Achillée millefeuille..... 148

Aconit anthora........ 6.3.3

Aconit bicolore. . . . . . . (tir)

Aconit napel......... 61

Aconit tue-loup ...... 62

Aconitum anthore. L. . . . 63

- lycoclomm. L. . . 62

- mapellus. L. .... 61

- variegatum. L. . 63

Atusa cymapium. L. . . . . 94

Airelle rouge......... 95

Ajuga reptans. L . . . . . 118

Alchemilla vulgaris. L. . . 82

Alchemille vulgaire..... 82

Althere officinalis. L ..... 72

Anchusa officinatis. L. . . . 133

Andromeda poliifolia. L . . 100

Andromède à feuilles de polinm 100

Anginecum flagrans. Du 'I'h. 50

Anlhriscus cercfolium. Hoff. 95

Anthrisque cerfeuil . . . . 95

Apinn graveolens. I. . . . . 91

Apocynérs. . . . . . 137

Araltackes. . . . . . . 89

Arbousier unedo........ 97

Arbutus rnedo. L. ..... 97

Arctostaphylos cusserole . . . 98

Arctostaphylos uva ursi.

Spreng. ........ 98

Arghel ......... 137

ARISTOLOCHIÉES . . . . . 58

Armoise absinthe $\ldots \ldots 147$
Armoise commmue. . . . 148

Limicr mumtuma. L. . . . 145

Arnique des montagnes. . . 145

Artemisia absinthium. L . . 147

- vulgaris. L ... 1.18

Arthante elongata. Miq. . . . 51

Asaret d'Europe....... is

Asarum europoeum. L . . . . .8

Asclepiadées ....... 190

Atropa belladona. L. ..... 1.5

Actraticeses...... T5

Aya-Pana . . . . . 150

Baguenaudier arbuste. . . . 80

Bardane commune. . . . . 143

Barosma betulina. Burt. . . . fir Barosma sematefolia. Will. . 68 Belladone médicinale. . . . 125 Berberidées. . . . . . 63 Berbéris commun ..... . 63 Berberis vulgaris. L . . . . (3;3) Betonica officinalis. L. . . . 118 Bétoine officinale...... 118 Boldo. . . . . . . . 54 Borraginées. . . . . . . 1:31 Borrago officinalis. L . . . 132 Bourrache officinale. . . . 1; Bucen litroe........ bi Bucco lnng. . . . . . . . 68 Buchu. . . . . . . . 67 Bugle rampant. . . . . . 118 Buglosse officinale. . . . 1833 Buis toujours vert. . . . . 57 Buxus sempervirens, L. . . 57 
Calmant aflicimale..... 11.

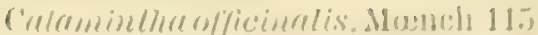

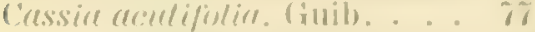
- ammestifolia. (inil).. i! - olmenlu. ('oll.... o j!

Cinser it lemillos ajgues. . . - ifoiles... o!! - olovios. i!

Cerrosus lomocerenses. L. . Sis

Corfenil.......... ge

Cerisier laturicl cerise.... $8: 3$

Charlon hénil ...... 14.1

Chicolée situvare ..... 15\%)

Chimaphilr mbellala. Nut. . 10?

Cichorium inlubes. L.... 1502

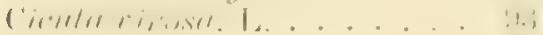

Cirue tachetée........ 133

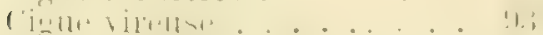

Citronnelle....... 10.

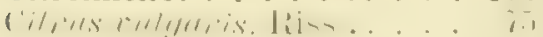

Cnicus benerlictus. L . . . . 14t

Cochleaina officinalis. I. .. 61

Cochleaire officinale. .... lil

Coluten nilwoinscens. I. ... 80

Conifires ........ 18

Composées. ....... 111

Conium mucuelatum. L ... . !):;

Coniaria muilifalia. L . . . (61

Coriaire il feuilles de myrte. . 61

Cortariées ...... 64

Consonde officinale .... 131

Cresson de fontaine..... 61

Cruchereres ......... 61

Cynoglosse officinale .... 135

Cynoglossum offuimale. L. . 13:3

1)atua stramoine .....127

Datura stramonium. I.... 127

Dicotrlenoses ...... 51

Digitale à grindes fleurs . . 123

Digitale pourprée ..... 1.2.2

Digitalis grondiflora. All. . . 123

Digitalis murpurea. L. . . . 122

DIPSACÉES ........ 110

Empleumum servalifolium. Ait. (is

Epine vinette. ....... 633

Ericinées.......... 96

ERITHRONYLEES. ..... TH

Ei"ylhroxylan coco. L.... rit

Ethuse ache des chiens . . . yt

Eucalyntus globulus. Lab. . . 86

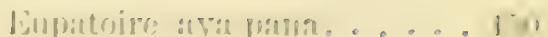

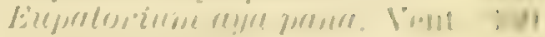

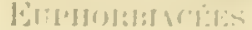

[ㄴ

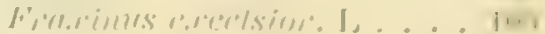

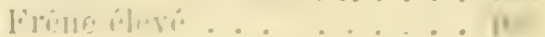

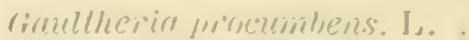
firtutherric cousleser.

fienevicle re Virerinie.

renurier sabins.

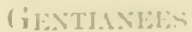

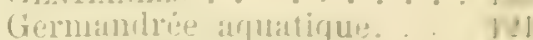

Germandrée vetit clüne. . 1:1

Glechonale lederacer.. L. . . . 11"'

Filechome lierre tripestre. . I 1

Giroseillier noir. . . . . . . -

firosstrmitres ........

filimaure ofticinale. .... :

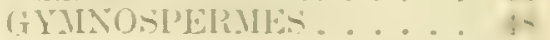

Hedera helia. L.........

Houx commull. . . . . int

Houx du Paraguay ..... ju,

IIyoscyanms albis. L. .... 1..1

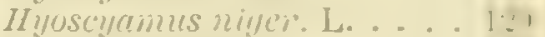

Hysope ofticinale ..... 111,

Hyssomus oflicinalis. L. . . 11..

lles aurifolium. I. ..... I11

Ilex juenguansusis. Lamb. Ihi,

ILICTEES . . . . . 10",

Imula connza. D. C. .... 14\%

Imula liclemimin. L. . . . . . 1:m

Inule atunce ........ 1,

[1,1]

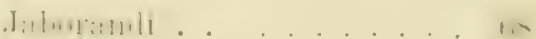

$1+1,1, \ldots \ldots$

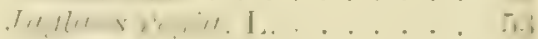

Jumipeirls sabina. L. . . . . is

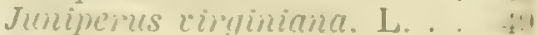

Jusquiame blanche ..... 1:iı

Jusquiame noire. ..... 1:1

Kimaphile en ombelle . . . 1,?

LAlilits .......... 109 Lappa communis. Cos. et Ger. 143

Laurier cerise...... 83

Laurier d'Apollon..... 59 
LATRINES . . . . . . 5!

Lmmins molilis. I. . . . . 5!

Ledon des marais. . . . . . 1111

Ledum palustie. L. .... 1111

Légumineuses. ....... i i

Lierre grimpant. . . . . . .

Ligurier citrisutertr. L. . . . 1018

Liphiar citromelle. . . . . 111.

Lestrelin influte. L. . . . . . 13:!

LOHELIA EE . . . . . . 1;:?

Malva rotundifolia. L. . . . is - syluestris. L. . . . . 愺

MALTACEES ........ T.

Marrube commun. . . . . 121)

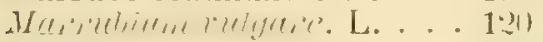

Maté ... ....... 110.

Matico . ....... . 51

Mauve it feuilles rondes. . . . i;

Mauve sauvage ........ . .

Melissa officinalis. L. . . . 4:3

Télisse officinale ..... $4: 3$

Menyanthe trèflle d'eau. . . 13.

Menyanthes trifoliata. L. . 1:i.,

Millefeuille. ...... 14

Molène médicinale. . . . . 12:;

MonIMTACÉEs. ........

MONOCOTYLEDONES. . . . . . 0

Morelle noire . . . . . . 12S

Mrrtacées . . . . . . 81j

Myrte commun ..... . 87

Myrtus communis. L. . . . . 8i

Nasturtium officinale. Bor. 61

Nepeta cataire. . . . . . 11t

Tepele cretritire. L. . . . . 114

Nicotiana mestica. L. . . . 1:31

- trelurcum. L. . . . 130

Nicotiane rustique. . . . . 1:11

- tabac....... 1:31)

Noyer commun ...... . 53

Enanthe à feuilles d'ache . . (1)

Enanthe apiifolia. Brot. . . (t)

OleinéES. . . . . . . 10:;

Ombillinenes. . . . . ! !

Oranger.......... 75

ORCHIDÉES ......... 50

Origan dictamme . . . . 11!

Origanem dictammes. L. . . 11!)

Oxilide oseille....... 71

OxALIDÉES........... il

Oxalis acetosella. L. . . . . 71
Parietaria officinalis. L. . . 56

l'ariétaire officinale. . . . 26

Pêcher commun....... 84

Persica vulgaris. Mill. . . 81

Persil cultivé. . . . . . 99

Perrenche it grandes tleurs. . 138

Pervenche naine. . . . . . 137

Petroselinum sativum. Hoff. 92

Pilocarpe à feuilles pennées. . 68

Pilocarpus pennatifolius.

Lem ......... 68

Piper reticulatum...... 53

Piperitées. . . . . . . 51

Pissenlit dent de lion .... 153

Plantaginées . . . . . 105

l'luntago lanceolata. L. . . 107

- major. L. .... 105

- media.L..... 106

Plantain à grandes feuilles. . 105

- Iancéolé ..... 107 moyen...... 106

Pneumes boldus. Mol ... 54

Poivre réticulé....... 53

Ptelea trifoliata. L...... 70

Ptelée à trois folioles. . . . . 70

Pelmonaria officinalis. L. . 131

Pulmonaire officinale. . . 134

Prrolacées . . . . . . 102

Redoul.......... 64

RENONCULACÉES . . . . . . 61

Rhus coriaria. L. ..... 76

- toxicodendron. L. . . 77

Rilues nitmm. L . . . . . \$s

Romarin officinal ..... 112

Ronce arbrisseau ...... 85

RosacÉES. . . . . . . 81

Rosmarinus officinalis. L. . 112

Rubus fruticosus. L . . . . 85

Rue fétide ....... 66

- de montagne...... 67

Ruta graveolens. L. . ... 66

- montana. L. . . . . 67

Rutacées ......... 65

Sabine ........... 48

Salvia officinalis. L. . . . . 111

- pratensis. L. . . . 112

- sclaren. L ...... 112

Sanicle d'Europe . . . . . 90

Sanicula europea. L. . . . 90

Saponaria officinalis. L. . 59

Saponaire officinale..... 59

12 
Sarrette des jardins . . . 117

Sintureir horlensis. I. .... 117

Singe officinale...... 111

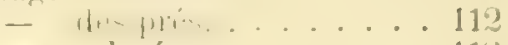

-.. sclarée . . . . . 11\%

scrubiosa succisn. I. . . . . 1.10

Scabieuse succise. . . . 140

SCHORIULARINÉES . . . . 1 12.

Scné........ T

Senecio rulgaris. L. . . . 1.45

Sénecon commun .. .... 145

Serionin jaborandi. Gaud. 52

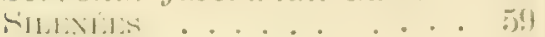

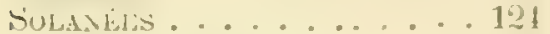

Solanum nignum. L. ..... 128

Solenosteme arghel. L. . . 13i

Stramoine........ 12r

Sumac des corroyeurs ... 76

vénéneux .....

Symplaylame officinale. L . . 181

SYAANTHÉRÉES . . . . . 141

Tilbile .......... 1:31

Tanacetum vulgare. L. . . 146

'Tanaisie commune .... 146

Taraxacum dens leonis. Desf. $15 ?$

'T'erebinthacees. . . . . 76

'lennstremacées ...... 73

Tencrium chamedrys. L. . . 121
Terovinar scriprliume I. . . 1:1

This de chine . . is

Therr chimensis, bind . i,

Theplerosin ngullinere. J). C.. i

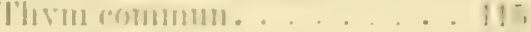

- serpolet....... II,

Thymens sermylleme. L . . iti. velgreis. L . . . 115

lussilage pas-dline .... 11:

- pétasite. . . . 1.,

Tussilago furfur $x$. I_ . . . . . i.:

- pelasiles. L. . . 1:!

Tylophora asthmaliere. Wien.

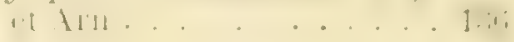

I

Li:1 $111: i \ldots \ldots . . \ldots$

VACCINIÉES ..........

Vaccinima vilis iden. L. . .

VERBASCEES . . . . . . 1 . .

Terbascruir nigireme. L . . . 1:4

- thrijsuses. L . . 1...:

Terbena officinalis. L. . . 1117

Verbentcees ....... 11\%

Verreine ofticinale..... I11

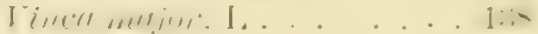

- mimo. L....... 1:i 


\section{EXPLICATION DES PLANGHES}

Les espèces đque nous allons désignées ont étẻ dessinées au grossissement de 135 diamètres, sauf indications spéciales.

\section{PLANCHE I.}

Fig. I. - Epiderme inférieur de miela conyza. A droite cellules de l'épiderme et de la nervure. A gauche cellules épidermiques à contour' sinueux de la lame, avec poils formés d'une rangée de cellules dont la supérieure, conique, est élargie brusquement à la base ; stomates.

Fig. II. - Epiderme supérieur du Tussilago farfara. Cellules à contour polygonal, revêtues à leur surface d'une cuticule présentant des crètes saillantes qui soffrent dans la figure sous la forme de stries.

FIG. III. - Coupe transversále du limbe de l'Artemisia absinthium. $a$, flinde. $b$, poil. Au-dessus et au-dessous épiderme.

FIG. IV. - Coupe transversale de la lame foliaire de Digitalis purpurea. $c$, poil à une rangée de quatre cellules dont la membrane présente de petits tubercules. $d$, glande surmontant un poil.

FIG. V. - Epiderme de Ptelea trifoitata. Cellules ì contour polygonal, présentant dans leur intérieur des cristaux d'inuline. Stomates elliptiques. Poils unicellulaires coniques.

FIG. VI. - Epiderme de Thymus vulgaris. Cellules à membianes ponctuées. Stomates elliptiques placés au milieu de cloisons sẻparant deux cellules.

\section{PLANCHE II.}

FIG. 1. - Epiderme neural de Glechoma helleracca, avec deux glandés et un poil conique dont la base est supportée par une éminence épidermique pluricellulaire.

Fí. II. - Epiderme neural de Cichorium intybus. Poil à plusieurs rangées cellulaires. G. ${ }^{90}$. 


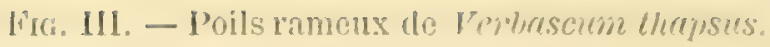

ling. IV. - Poils lamificis de lessmariunes.

líg. V. - Epiderme inféricur de Ihhes cortaria. 'Trois poils ralandnleux.

lira. VI. - Portion de feuille de Lawpre major. Epiderme muni dr: deux glandes formées de denx séries cellulaires.

Frg. VIL. - Epiderme de symphylum officinale avee des proils ulauduleux unicellulaires surmontés d'une glande arrondie et avec des yoils: unicellulaires en hameron.

Fia. VIII. - Epiderme d'Alropa belladona. Poils glanduleux.

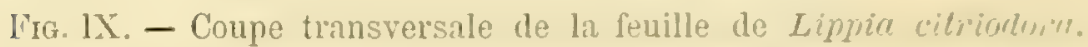
au-dessus et au-dessous sont de grosses glandes dont une est situie dans une dépression épidermique.

FIG. X. - Epiderme neural de Pulmonario offeinalis arec trois poils glanduleux.

FIG. XI. - Portion de nervure et de lame, de Ribes nigmun. Sur la lame et contre la nervure est une grosse glande pluricellulaire. Au-dessus de l'amas cellulaire se troure de l'huile essentielle qui soulève de la cuticule.

\section{PLANCHE III.}

FiF. I. - Epiderme de la nervure de Tanacelum rulgare. Poils gran. duleux

Fig. II. - Epiderme de la nervure de Salvia pralensis. Cellules épidermiques ponctuées. $b, b^{\prime}$, Poils glanduleux à pédicelle moyen bicellulaires. $a, a$, poils glanduleux.

FIg. III. - Epiderme de Plantago lanceolata arec deur poils glan. duleux.

Fig. IV. - Epiderme de Scabiosa succisa. Cellules épidermirues ponctuées, deux poils glanduleux.

FlG. V. - Coupe transversale du limbe de la feuille de Altha officinalis. $a$, poils glanduleux. $u^{\prime}$, poils en rosette.

Fig. VI. - Epiderme de Colutea arborescens. Cellules ípidermiques sinueuses. Poils unicellulaires.

FIG. VII. - Epirlerme de Dahura shramonimm. Stomates poils glanduleux. 
FIG. VIII. - Epiderme de la nervure de la feuille de Hyoscyamus. Poils simples glanduleux à pédicelle long.

\section{PLANCHE IV.}

FIG. I. - Coupe transversale une feuille de Boldo. e, épiderme supérieur. $d$, couche de renforcement. $c$, parenchyme en palissade. $b$, parenchyme lacuneux contenant deux glandes unicellulaires $a$.

FIG. II. - Coupe transversale de la coupe d'Oranger. c, parenchyme en palissade. $d$, parenchyme lacuneux. $b$, cellule ì cristaux isolés. $a$, glande pluricellulaire.

FIG. III. - Coupe transversale de la feuille d'Eucalyptus glotulus. $a$, parenchyme en palissade supérieur. $c$, parenchyme en palissade inférieur. Entre les deux, du parenchyme lacuneux renfermant des celIules à cristaux agglomérés (c). Cellules à cristaux isolés.

FIG. IV. - Coupe de la feuille de Thea chinensis, a, cellules épaissies, lignifiẻes, rameuses.

FIG. V. - Coupe de la feuille de Salvia. Cellules du parenchyme vert,

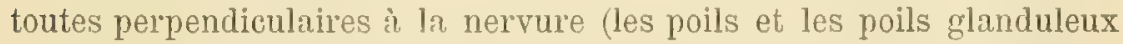
n'ont pas étẻ dessinés).

FIG. VI. Coupe de la feuille du Cassia acutifolia. Mésophylle hétérogène, symétrique. $a$, parenchyme en palissade supếlieur. $c$, parenchyme en palissade inférieur, 0 , parenchyme lacuneux.

FIG. VII. - Coupe de feuille de Parietaria officinalis, a, cystolithe.

HIG. VIII. - Coupe de feuille d'Oxalis acetosella.

\section{PLANCHE V.}

Frg. I. - Coupe transversale de la feuille d'Ilex aquifolium. a, Epiderme supérieur. $b$, Assise de renforcement. $c$, deux couches de parenchyme en palissade, l'inférieure contient une cellule cristalligène. $d$, parenchyme lacuneux.

Fig. II. - Feuille de Mclissa officinalis. a, Epirlerme supérieur. b, Parenchyme en palissade. c, Tissu lacuneux. (Les poils glanduleux n'ont pas été représentés.)

Fra. III. - Feuille de Cochlearia. a, Epiderme supérieur. b, Parenchyme en palissade ì trois couches. $c$, Tissu lacuneux. 
Fa. IV. - Coupe transiersale du bord foliaire d'IJyougames allus. a, Parenchyme vert en palissade. 1, Epiderme. ce cellule cristallipines.

Fra. V. - Coupe transversale du bord foliaire de Vaccintum vitisideca. $a$, Collules épaissies. $b$, Tissu en palissade.

Fia. VI. - Coupe transversale d'Atrona belladona. b, Eipiderme supérieur' $a$, Poil glanduleux. c, 'lissu en palissarle. $d$, Cellules renfer. mant des cristaux pulvérulents.

IIG. VII. - Feuille d'angrecum fragrans, traitée par la potasse. Deux épidermes contre chacun desquels s'appuie l'hypoderme. $u$, P'aren. chyme vert uniforme. $a, a^{\prime} a^{\prime \prime} a^{\prime \prime \prime}$ Eléments épaissis.

FIG. VII bis. - Feuille de Faham, traitée par cau bouillante (mêmes lettres que le précédent).

FIG. VIII. - Lambeau de tissu lacuneux de Saricula Europen.

FIG. IX. - Feuille de Jaboranai. a, Epiderme supérieur recourert de cuticule épaisse. $b$, Parenchyme en palissade. ce, Cellules í cristaux. $a$, Glande pluricellulaire. $e$, 'Tissu lacuneur.

\section{PLANCHE. VI.}

Fig. I. - Coupe transversale du Buxus sempervivins. a, Epiderme supérieur. $b$, parenchyme inférieur. $c$, fibres libériennes inféricures. $d$, liber celluleux. $e$, bois. $f$, cellules médullaires. $g$, fibres libériennes supérieures. G. .

FIG. II. - Coupe transtersale de la nervure principale de Coriaria myrtifolia. $a$, epiderme inférieur. $b$. parenchyme inférieur. $c$, gaine protectrice des faisceaux. $d$, liber. $e$, bois. $f$, hypoderme. $g$, épiderme supẻrieur. $\mathrm{G}_{i}^{0,}$.

FIG. III. - Coupe transversale d'une nervure, d'Alium graveolens. $a$, épiderme supérieur. $h$, hypoderme. $d$, parenchỹme supérieur. $c, g$, canaux secréteurs, $c$, bois. $f$, liber. $h$, hypoderme inférieur. .

FIG. IV. - Coupe transrersale de nerrure prinsipale 'd'Ura ursi. $a$, épiderme inférieur. $b$, parenchyme inférieur. $c$, liber celluleux. $d$, bois, $e$, cellules épaissies hypoderme. f, épiderme supèrieur.

FIG. V. - Système libéro-ligneux d'Atropa bellad Jna. c, bois. 0 , ilot de liber inférieur. $a$, rangée cellulaire entre les îloţ. $a$, illots libériens 
supérieurs. $e$, cellule de parenchyme supérieur. $f, g$, cellules à cristaux pulvérulents.

FIG. VI. - Coupe transversale de nervure de Menyanthes Irifoliata. $\alpha$, épiderme supérieur. $v, a, f$, faisceaux libéro-ligneux. $e$, parenchyn.e à grandes lacunes. $c$, épiderme inférieur. G. $\stackrel{s}{1}_{1}$.

\section{PLANCHE VII.}

FIG. I. - Coupe transversale de nervure de Malra. $a$, parenchyme inférieur. $d . b$, collenchyme inférieur. $c$, cellule cristalligène. $c$, liber. $f$, bois. $g$, moelle. $i$, collenchyme supérieur. $h$, épiderme supérieur. G. ' .

FIG. II. - Coupe transversale d'une portion de nervure de Arthante elongala. a, Epiderme inférieur. b, flôts de collenchyme. c, Parenchyme. $d$, Glandes unicellulaires dans le liber. $c, f$, Bois. $g, g^{\prime} g^{\prime \prime}$ Cellules épaissies ponctuées. G. .

Fig. III. - Coupe transversale de nervure de Rhus toxicodendron. $a, a^{\prime} a^{\prime \prime}$, canaux secréteurs dans le liber des faisceaux. $b$, bois. $c$, liber. $d$, parenchyme. $e$, hypoderme inférieur. $f$, épiderme inférieur. $g$, bois. $h$, liber. $i$, canal secréteur. $j$, parenchyme supérieur. $k$, hypoderme supérieur. $m$, épiderme supérieur, G. $\frac{9 ?}{7}$.

Fig. IV. - Coupe transversale de nervure de Berberis. $a, a^{\prime}$, liber. $c$, bois. $b$, masse à cellules lignifiées dans laquelle siègent les faisceaux. $c$, épiderme inférieur. $\alpha$, parenchyme inférieur dont les cellules ont une membrane cellulosique. $f$, Parenchyme à cellules lignifiées. g. Hypoderme supérieur. $h$, Epiderme supérieur. G. "?o.

Fig. V. - Coupe transversale d'un faisceau foliaire de Tussilago farfara. $a, g$, portion de tissus de remplissage. $b$, bois. $c$, liber celluleux. $a$, liber fibreux. $e$, $\dot{c}$, canaux secréteurs. $f$, liber supérieur fibreux.

\section{PLANCHE VIII.}

Fig. I. - Coupe transversale de nervure de Vinca minor. a, fibres libériennes, $b$, vaisseaux laticifères. $c$, épiderme supérieur. $d$, hypoderme inférieur. $e$, liber celluleux. $f$. bois. $g$, liber supérieur celluleux, $h$, illots de fibres libériennes. $m$, parenchyme supérieur. $n$, hypoderme supérieur:?o, épiderme supérieur. G. $\frac{9 n}{1}$.

FIG. II. - Coupe transversale de nervure d'Aconitum napellus, ' $a$, 
epiderme. "W, hypoderme. c, parenchyme infericur. d, libre bi-convere. $c$, bois. f, parenchyme supérieur. g, épiderme supérieur. h, grarenchyme supéricur. G. .

Fig. Ill. - Coupe transversale d'une feuille de Jmiperes sabina. $a$, épiderme supérieur. $b$, parenchyme supérieur. c, bois, e, liber. f, hy. poderme. $\%$, parenchyme vert. $h$, réscrvoir is résine.

Lig. IV. - Portion de coupe longitudinale de la feuille de Juniperns saluina. $a$, fibres réticulés. $b$, fibres ì ponctuations aréolées. $c$, cellules a ponctuations aréolées. G. ju,

líg. V. - Coupe longitudinale de nervure de Verlascum. $a, a^{\prime}$, poils desséchés incomplets. $v$, épiderme. $d, c$, hypoderme. $a, c$, parenchyme inferieur incolore. $r$, cellules it chlorophylle. $g$. liber celluleux; entre $g$ et $f$ fibres libériennes. $h$, vaisseaux ponctués. $o$, vaisseaux spiraux et trąchés. p, parenchyme supérieur. G. ${ }^{90}$. 


\section{Planche I.}

Iig. 1
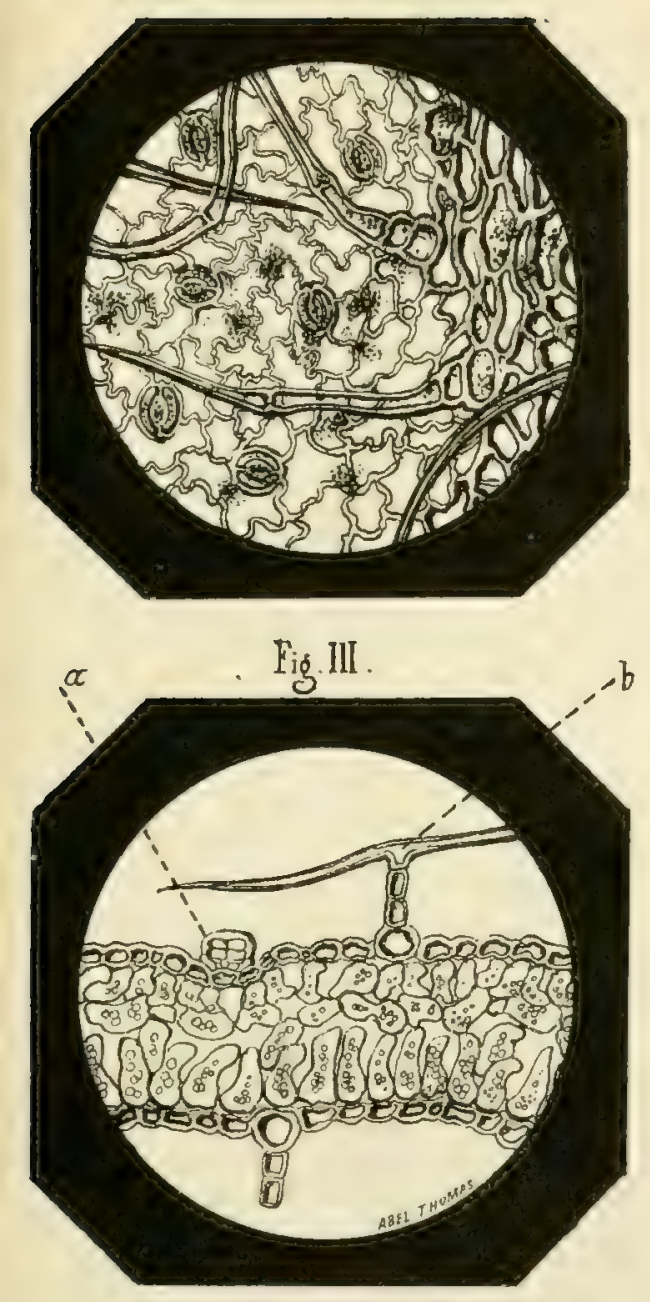

Fig. V.

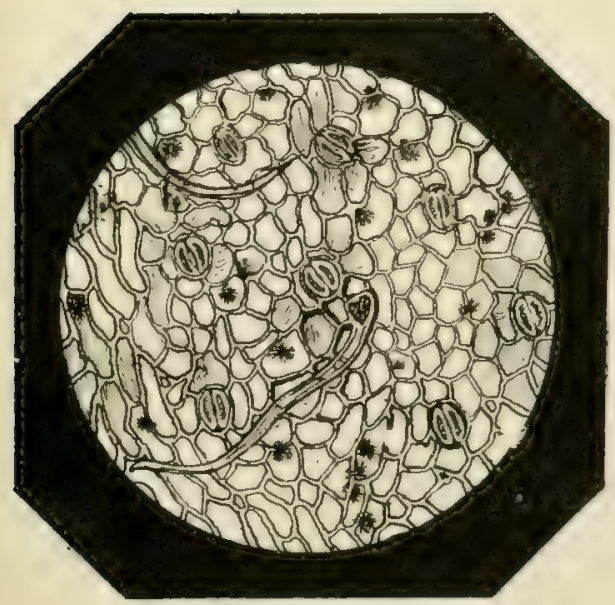

Fiog II
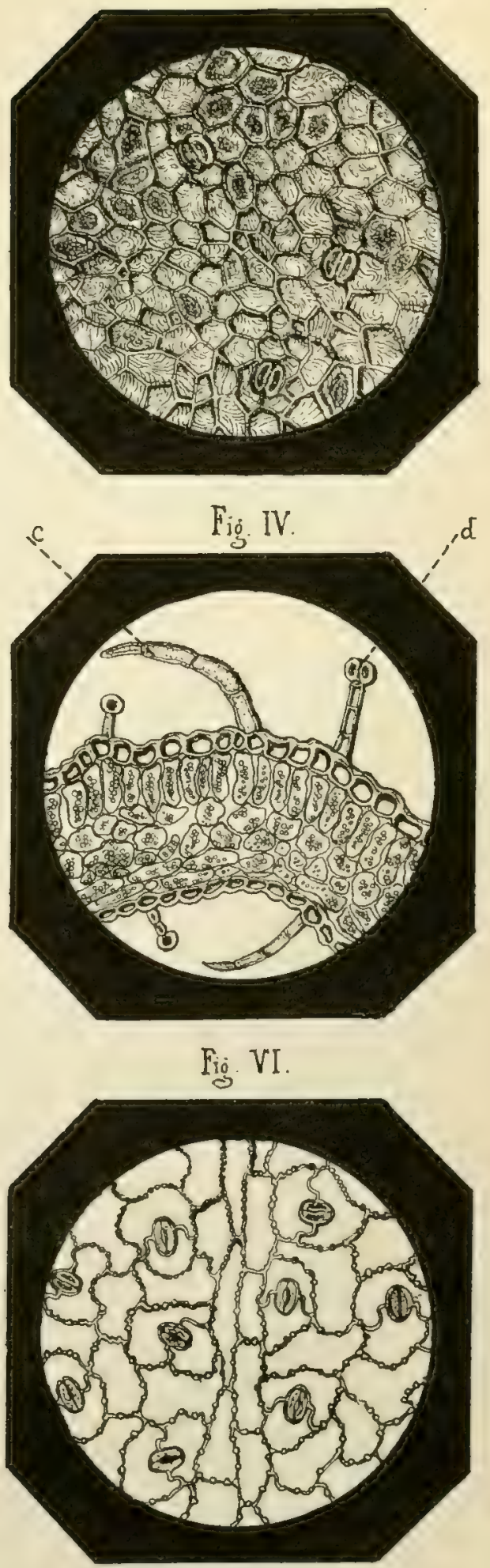

Ab. THOMAS. ad nat del. 

Fig I

Fig II

Fig III
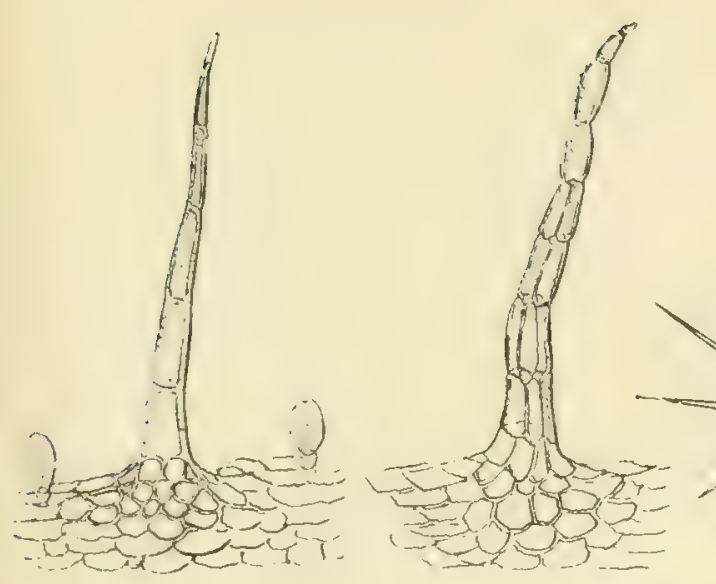

(1)

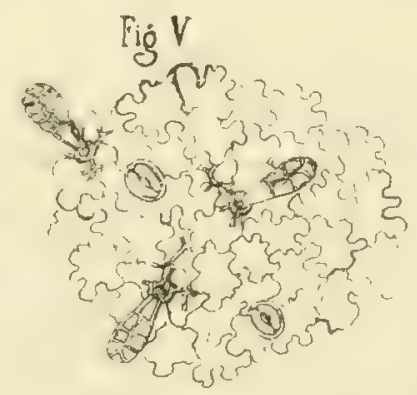

Fio VII

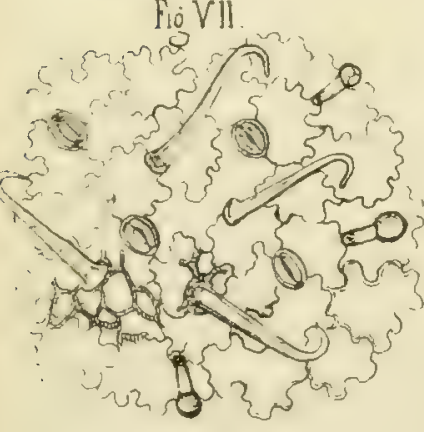

Fig Vill

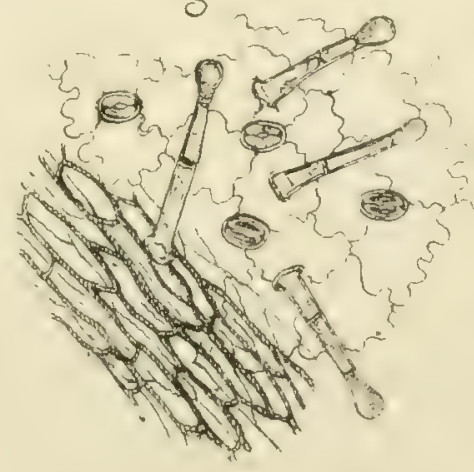

Fiog VI.

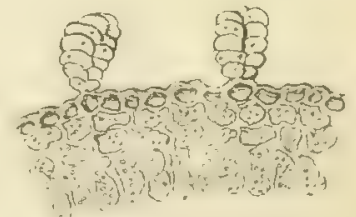

Figlx.

Fid.
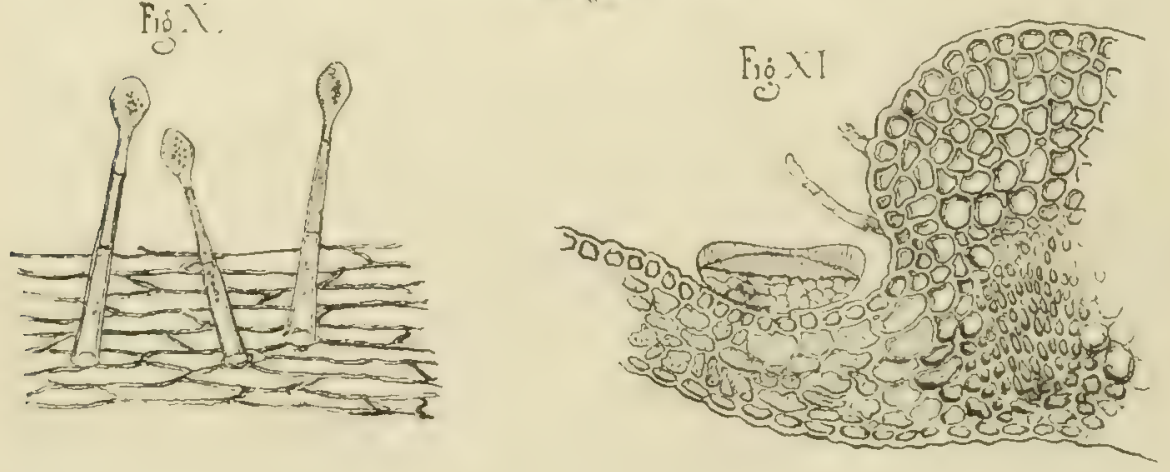

Ab.THOMAS ad.nat del.

Lith. H. CHRISTOPHE. Nancy 



\section{Planche III}

Fig. I
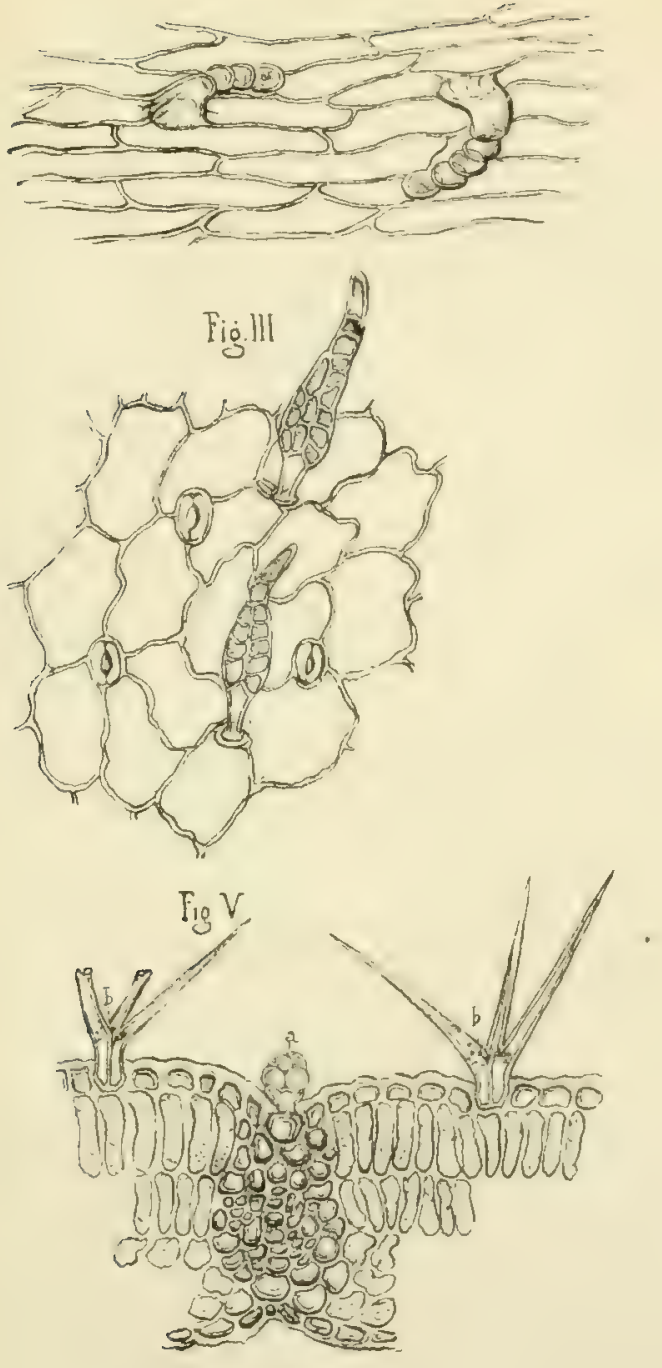

Fiog. VIl

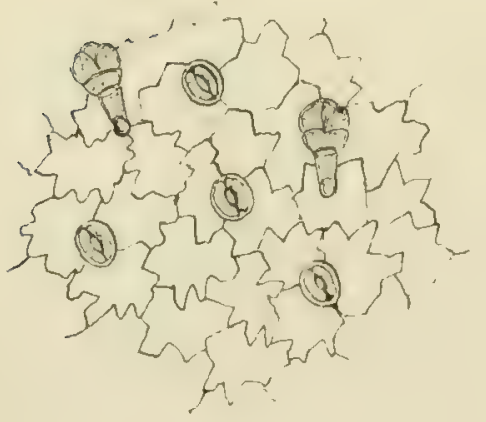

AbTHOMAS, ad nat del
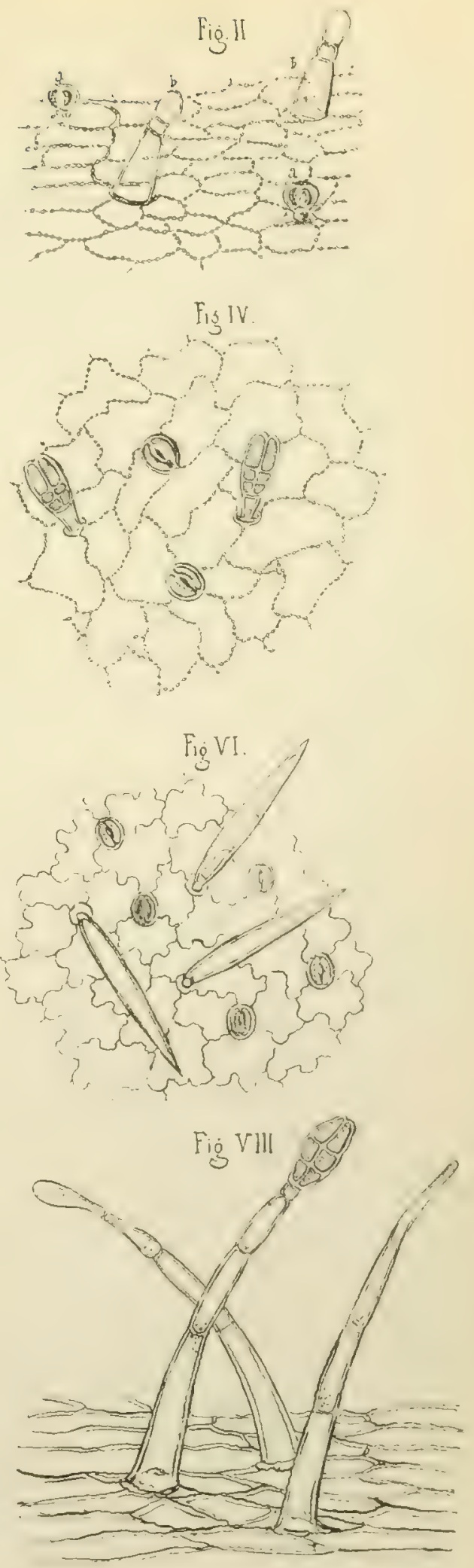

Lith.H.CHRISTOPHE. Nancy. 



\section{Planche IV.}

Figi I

Fiv 11

$2^{2}$
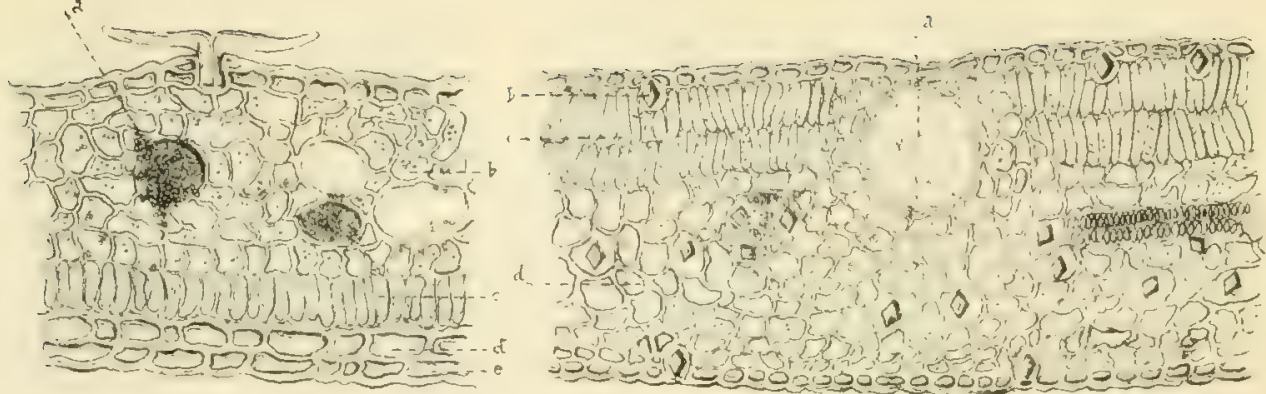

Fio 111

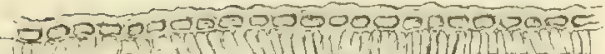

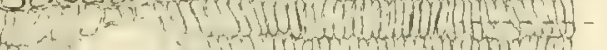

I.: 11

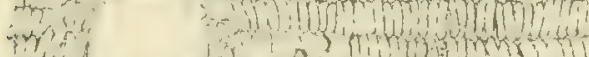

Iffin

(1)

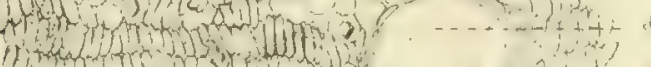
19.

(4)

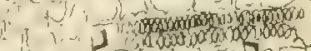

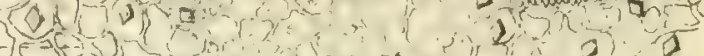
I2 20 a

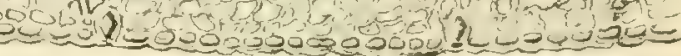

\section{V V}
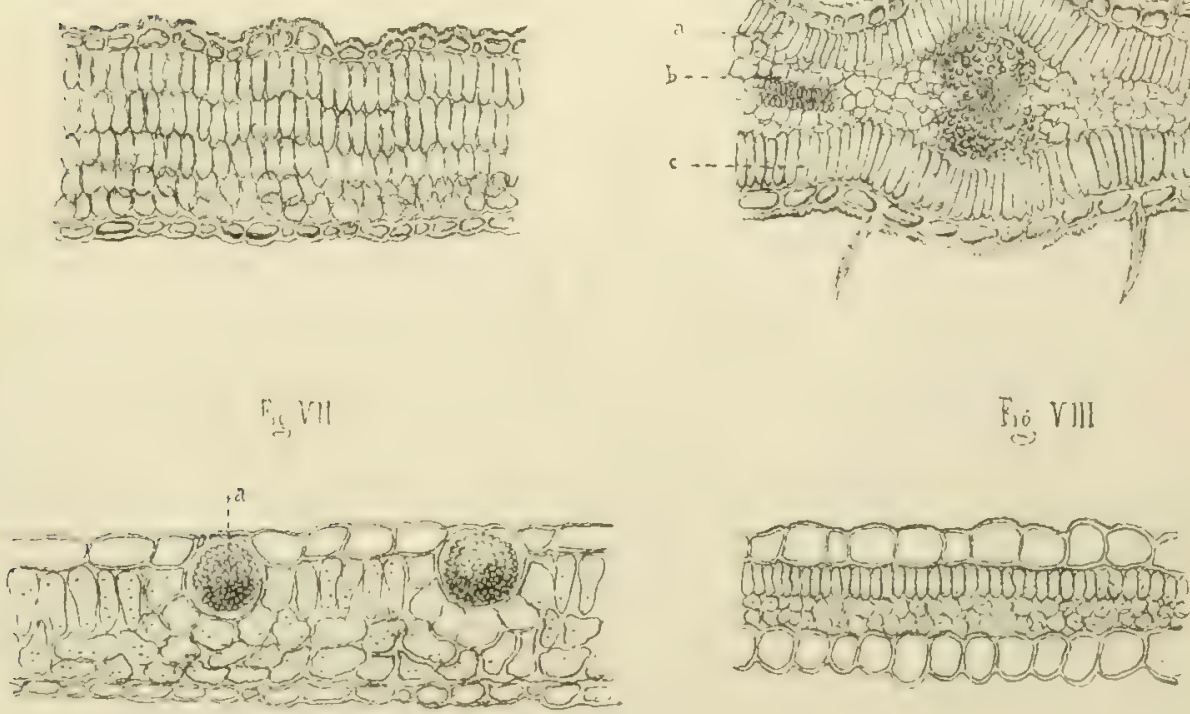

Fio. 1
Fiğ . II

Fiğ II

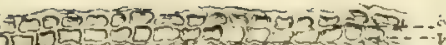

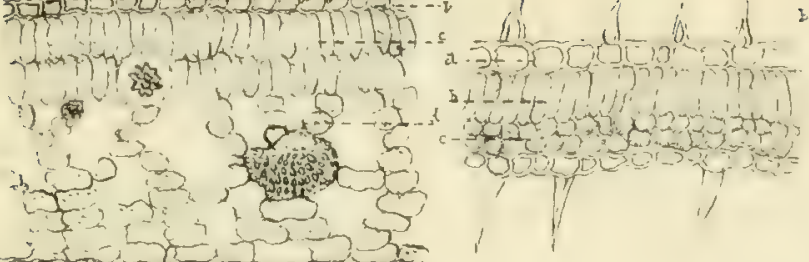

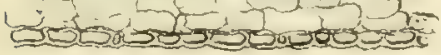

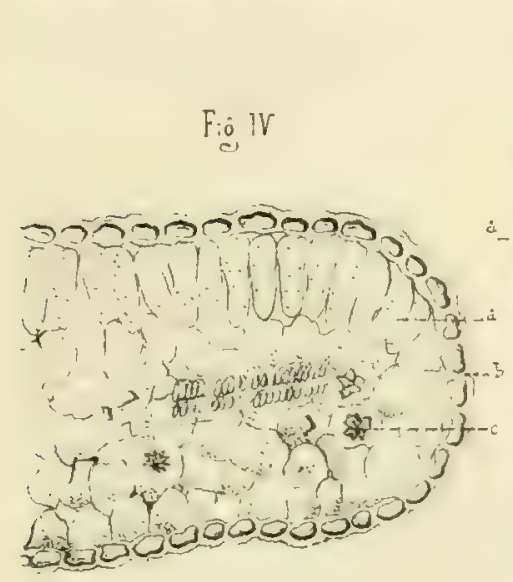

Fio. VII Lis.

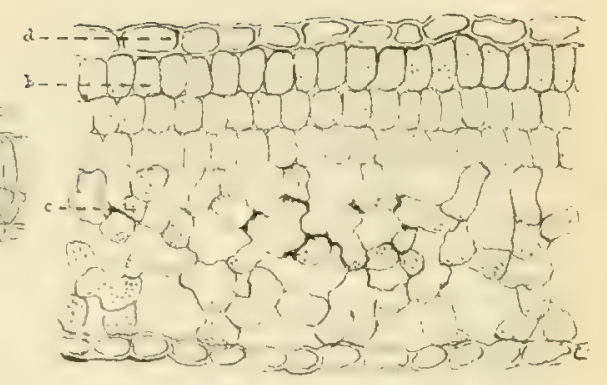

F:0 IV

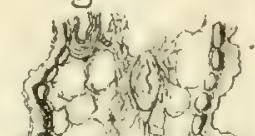

Fió $V$

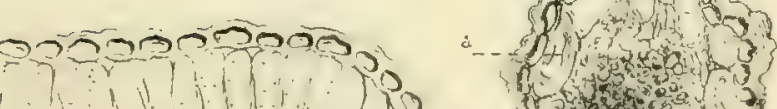

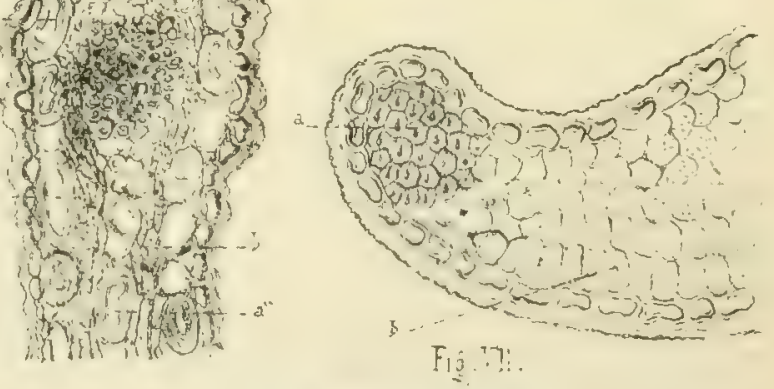

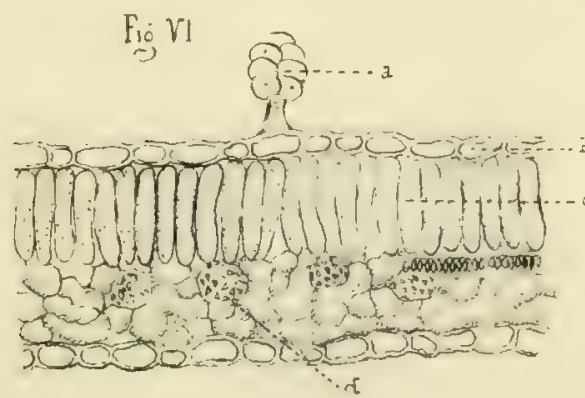

Z.o VIII

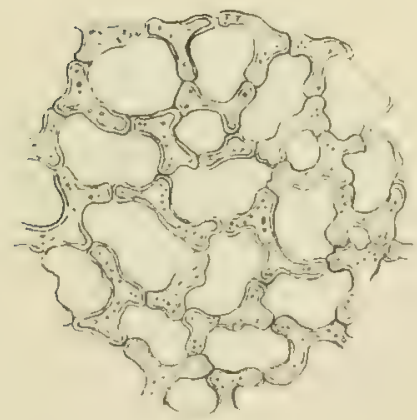

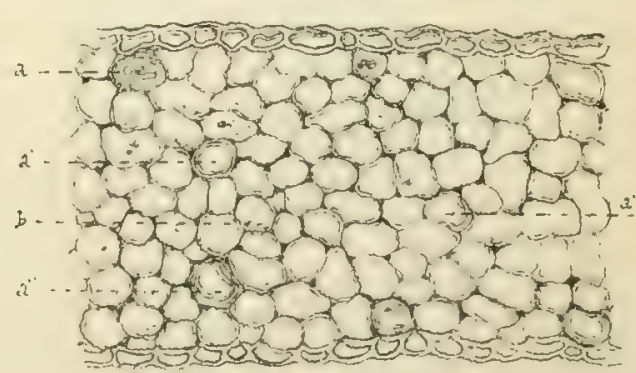

$\left.F_{10} ! 1\right]$

5

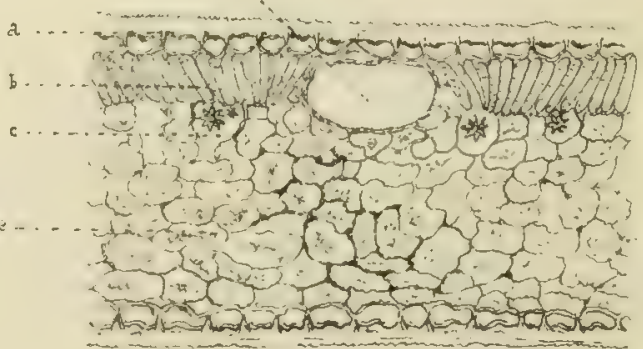



$F_{i j} I$

Planche VI.

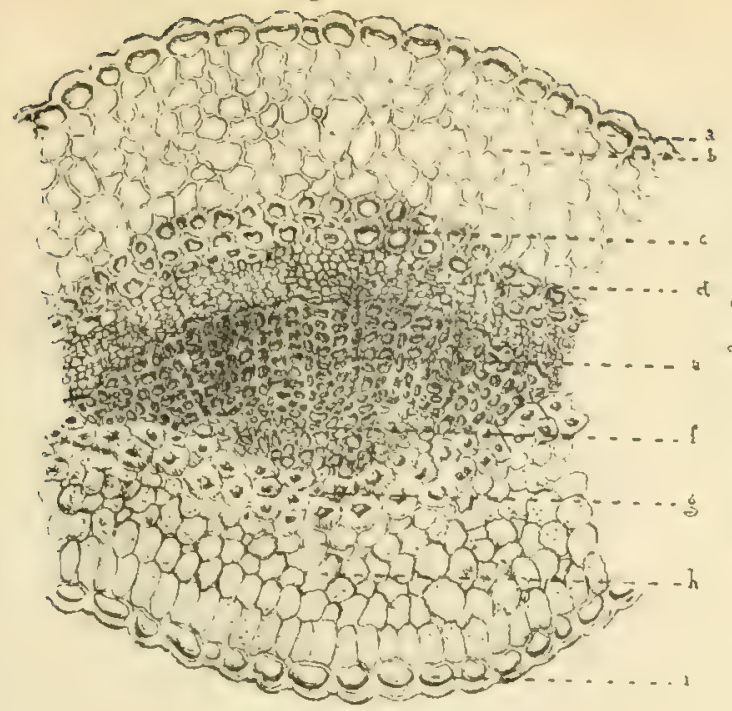

His if

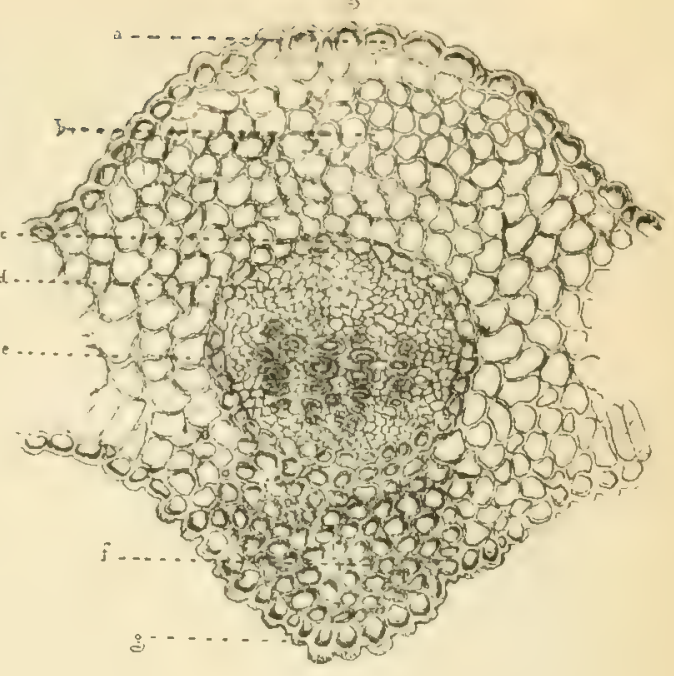

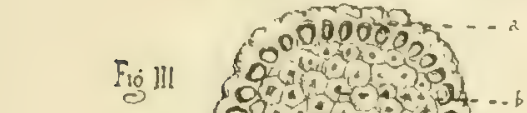

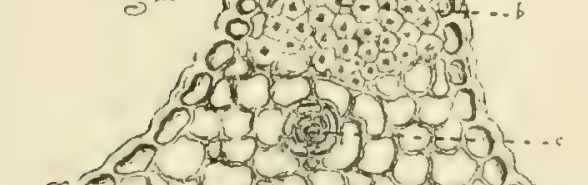

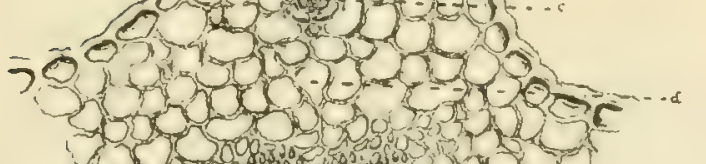

Q 203010

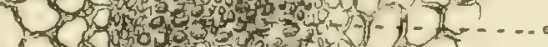

(1) 30 .

$00-123013-3=-i$

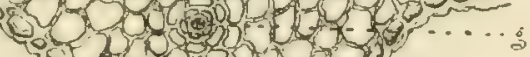

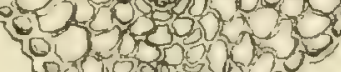

Qun a n

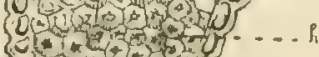

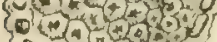

(- 4) 0 .

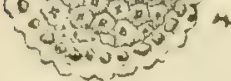

$\mathrm{Fig}_{\mathrm{ig}}: \mathrm{r}$

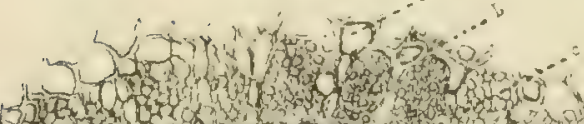

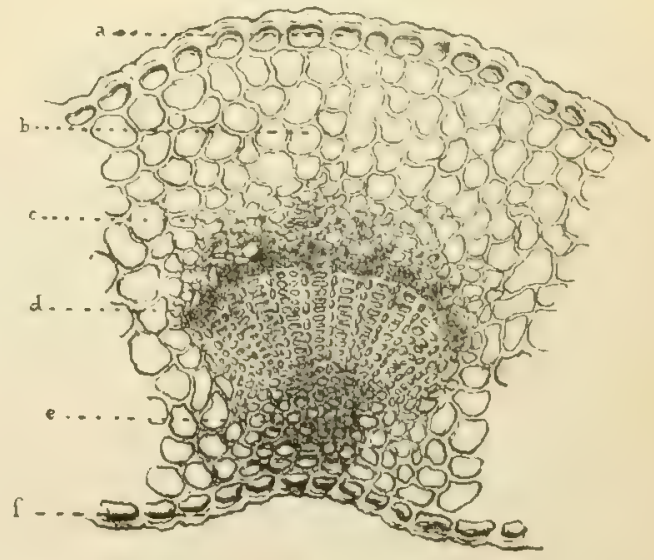

Fió $\mathrm{V}$

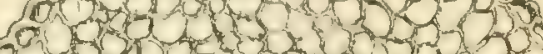

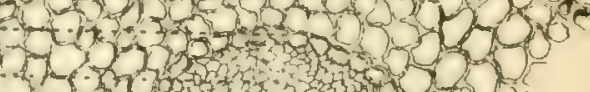

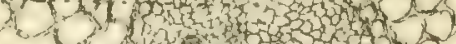





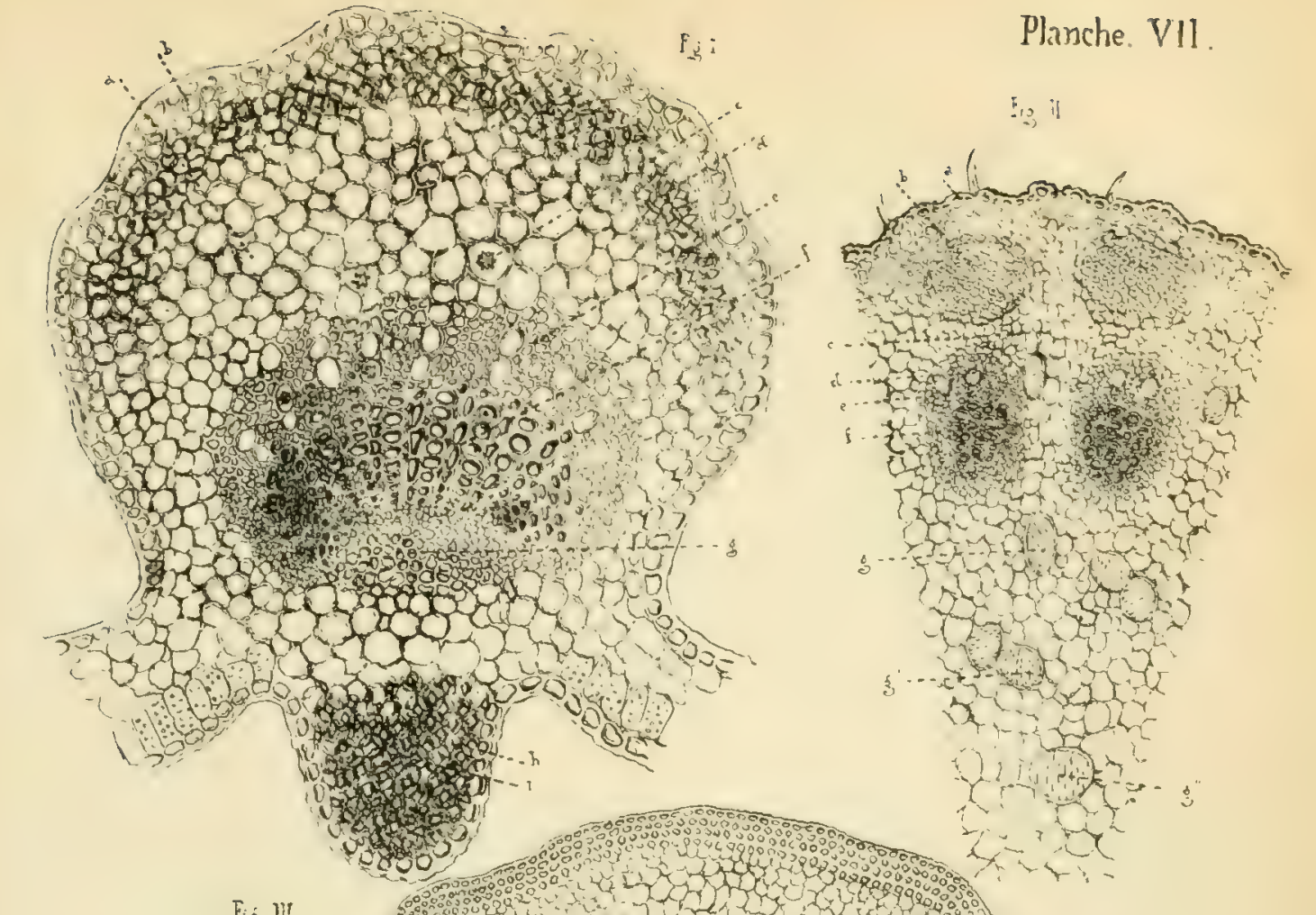

Fig. III.

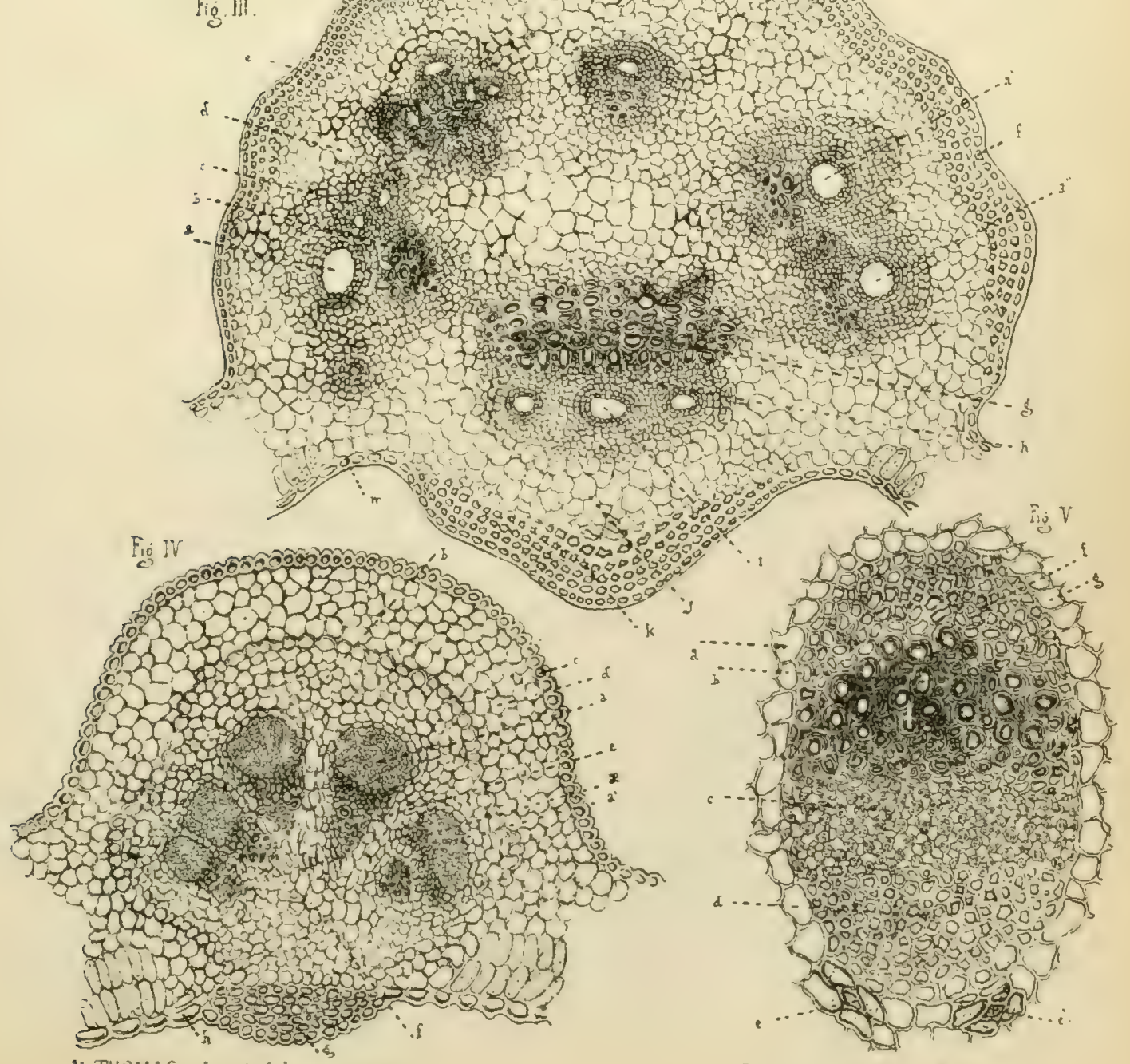

Ab. THUMAS ad nat del. Siltee Iers.

Iith H.CHRISTOPHE Kancy 

$5-7,-x+2$

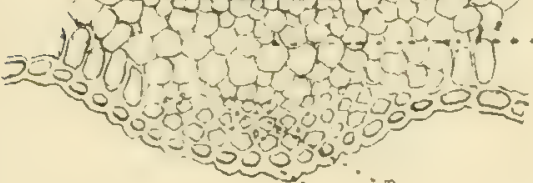

Fic. 111
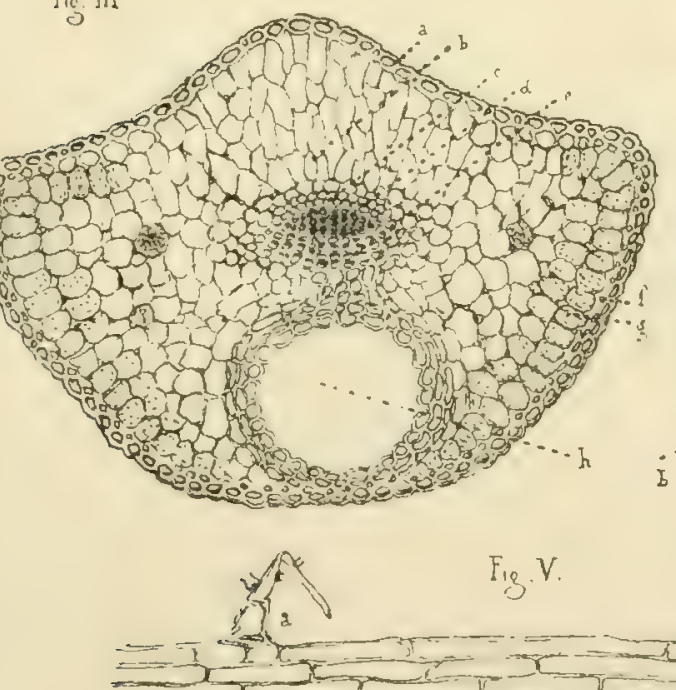

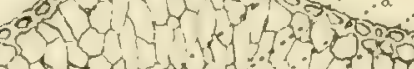

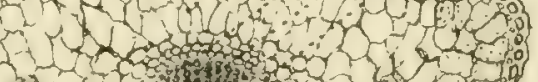
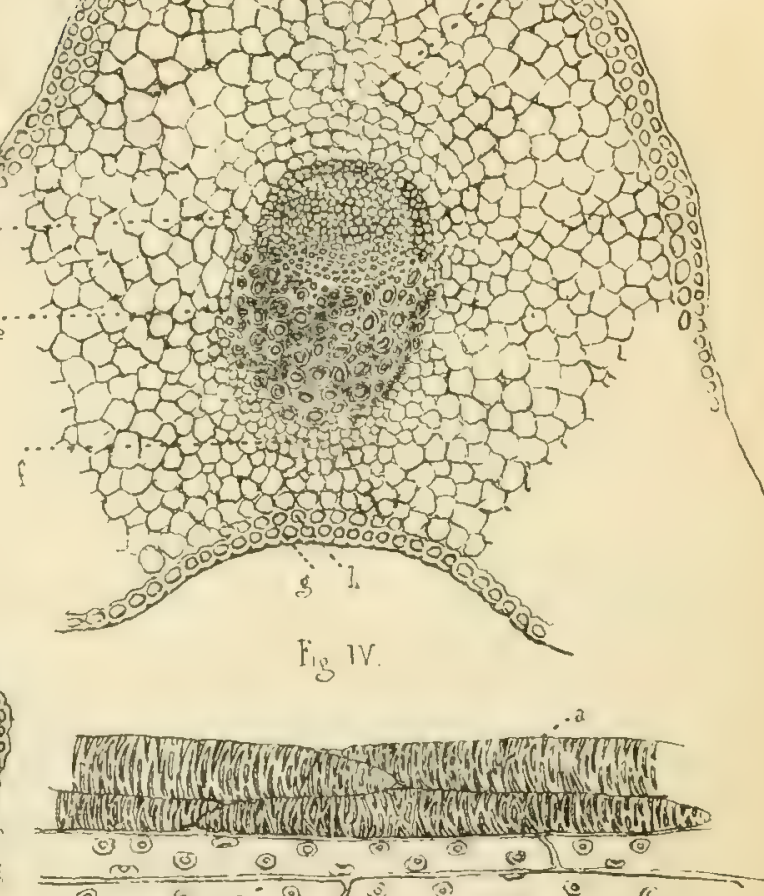

T. 3 .

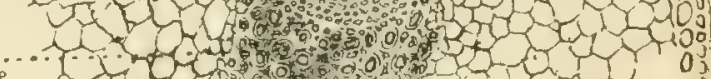

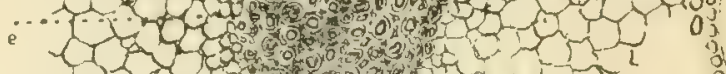

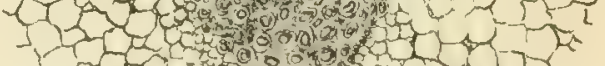
Inan war
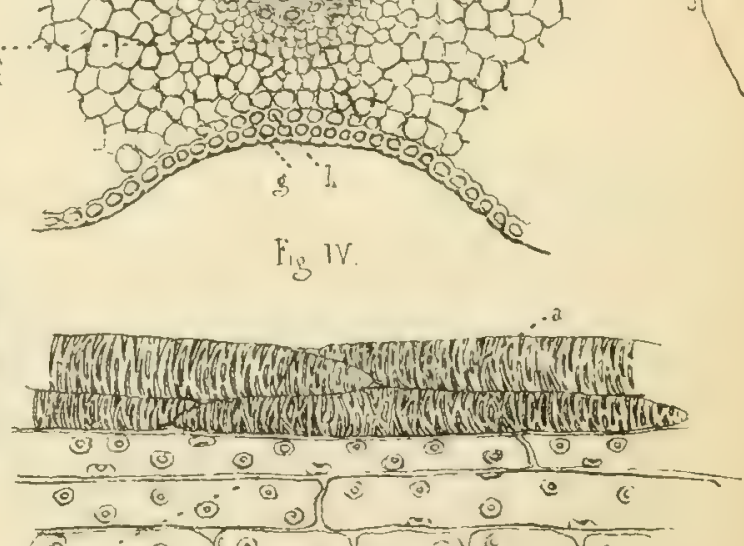

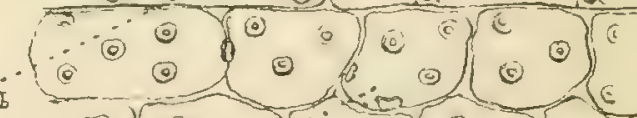

$$
\begin{aligned}
& \text { ०) } 0.0)[0]
\end{aligned}
$$<smiles>[Te]=C1CC1</smiles>

$F_{18}, V$

5 \&

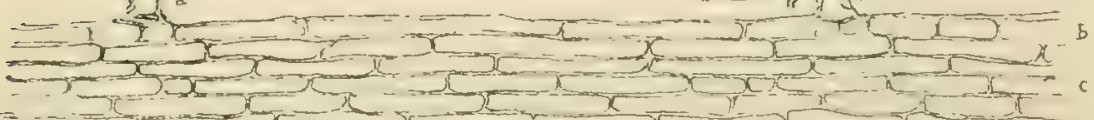

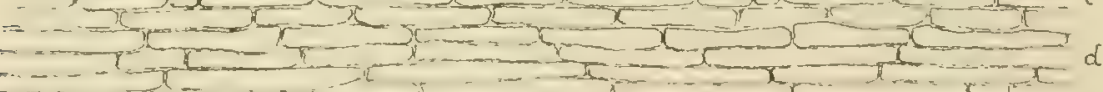

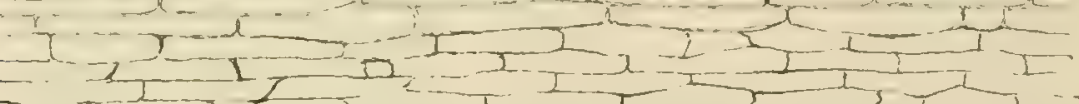

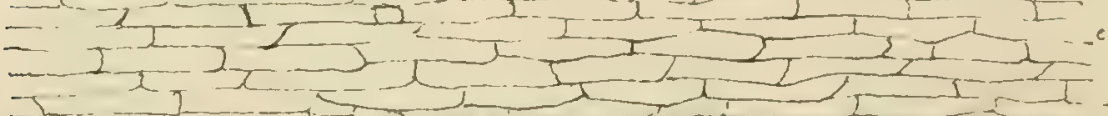

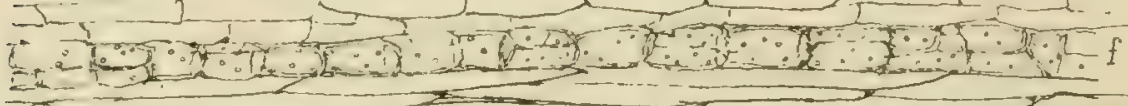

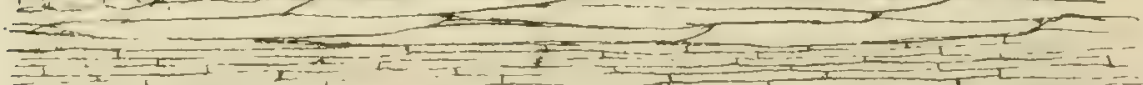

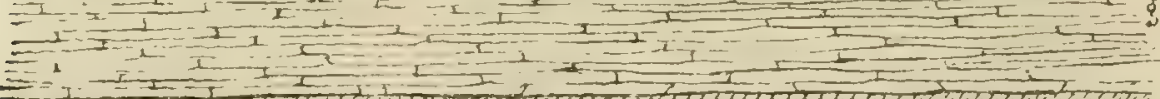

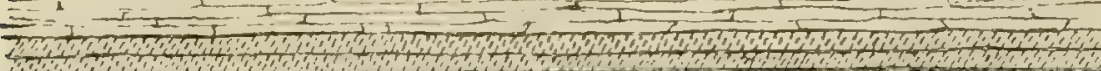

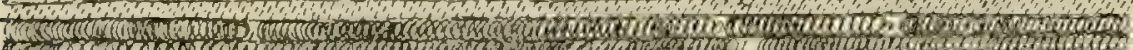

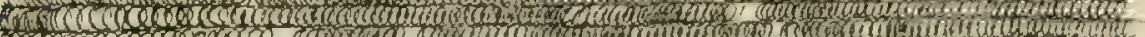

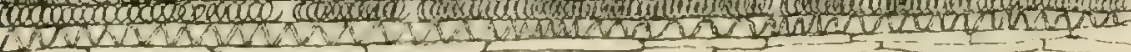

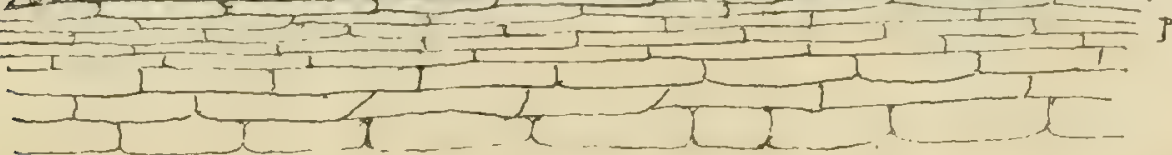











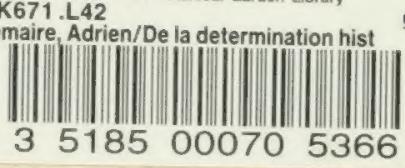


